\title{
A Grid-Adaptive Algebraic Hybrid RANS/LES Method
}

\author{
Dissertation \\ zur Erlangung des mathematisch-naturwissenschaftlichen \\ Doktorgrades \\ "Doctor rerum naturalium" \\ der Georg-August-Universität Göttingen \\ im Promotionsprogramm "Math Sciences" \\ der Georg-August University School of Science (GAUSS)
}

vorgelegt von

Silvia Reuß

aus Freiburg

Göttingen, 9.11.2015 


\section{Betreuungsausschuss}

Prof. Dr. Gert Lube, Institut für Numerische und Angewandte Mathematik, Universität Göttingen

Dr. Dieter Schwamborn, Abteilung $\mathrm{C}^{2} \mathrm{~A}^{2} \mathrm{~S}^{2} \mathrm{E}$,

Deutsches Zentrum für Luft- und Raumfahrt e.V.

Dr. Tobias Knopp, Abteilung $\mathrm{C}^{2} \mathrm{~A}^{2} \mathrm{~S}^{2} \mathrm{E}$,

Deutsches Zentrum für Luft- und Raumfahrt e.V.

\section{Mitglieder der Prüfungskommission}

\section{Referent:}

Prof. Dr. Gert Lube, Institut für Numerische und Angewandte Mathematik, Universität Göttingen

\section{Korreferent:}

Prof. Dr. Rolf Radespiel, Institut für Strömungsmechanik, TU Braunschweig

\section{Weitere Mitglieder der Prüfungskommission:}

Prof. Dr. David Russel Luke, Institut für Numerische und Angewandte Mathematik, Universität Göttingen

Prof. Dr. Ingo Witt, Mathematisches Institut, Universität Göttingen

Prof. Dr. Carsten Damm, Institut für Informatik, Universität Göttingen

Prof. Dr. Andreas Tilgner, Institut für Geophysik, Universität Göttingen

Tag der Mündlichen Prüfung: $\quad 16.12 .2015$ 


\section{Danksagung}

Zunächst gilt mein ganz besonderer Dank meinem Doktorvater Prof. Dr. Gert Lube. Unsere Zusammenarbeit empfand ich als sehr motivierend, da er bei seiner Betreuung genau das richtige Maß zwischen forderndem Ansporn und Freiraum gefunden hat. Besonders für die schriftliche Ausarbeitung hat er immer wieder wertvolle Ratschläge und Anstöße gegeben. Darüber hinaus möchte ich mich bei meinem Zweitberichter Prof. Dr.-Ing. Rolf Radespiel herzlich bedanken. Als Projektleiter der DFG Forschergruppe, die den Forschungsrahmen meiner Dissertation bildete, hat er die Arbeit während der gesamten Laufzeit konstruktiv begleitet.

Weiterhin gilt mein Dank meinen Kolleginnen und Kollegen aus dem DLR, insbesondere meinem fachlichen Betreuer Dr. Tobias Knopp und meinem Abteilungsleiter Dr. Dieter Schwamborn. Für den hervoragend gelungenen Zwei-Element-Doktorhut und die Fahrt im festlich geschmückten Doktorwagen bedanke ich mich bei Alexis, Baptiste, Daria, Julian, Manli, Marco und Michael.

Nicht zuletzt bin ich meiner Familie dankbar, die immer für mich da war und sogar $\mathrm{zu}$ meiner Verteidigung angereist ist, um mich moralisch zu unterstützen.

Und schließlich bleibt noch eine ganz besondere Person zu nennen. Als Kollege hat Dr. Axel Probst diese Arbeit in unzähligen fachlichen Diskussionen unterstützt. Als mein Lebensgefährte hat er mir den nötigen Rückhalt geboten, wann immer es notwendig war. 



\section{Contents}

Introduction $\quad 1$

Overview over Publications ...................... 3

1 Turbulence: physical background and modeling $\quad 7$

1.1 Compressible Navier-Stokes equations . . . . . . . . . . . . . . . 7

1.2 Description of turbulence . . . . . . . . . . . . . . . 9

1.3 Numerical simulation of turbulent flows . . . . . . . . . . . . . 10

1.3.1 Statistical turbulence modeling - RANS . . . . . . . . . . . 11

1.3.2 Large-eddy simulation - LES . . . . . . . . . . . . . . 13

1.3.3 Hybrid RANS/LES modeling . . . . . . . . . . . . . . 15

2 Numerical method: DLR-TAU 21

2.1 Discretization of the computational domain . . . . . . . . . . . 21

2.2 Discretization of the governing equations . . . . . . . . . . . . 22

2.3 Optimization of the numerical scheme for scale-resolving simulations . 25

2.4 Convergence criteria and averaging . . . . . . . . . . . . . . 27

2.5 Chimera method and deformation tool . . . . . . . . . . . 28

3 A grid-adaptive algebraic hybrid RANS/LES model 31

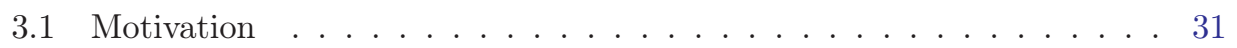

3.2 Grid adaptation . . . . . . . . . . . . . . . 34

3.2 .1 Span-wise grid adaption . . . . . . . . . . . . 34

3.2.2 Grid-resolution sensors . . . . . . . . . . . . . . 35

3.3 Algebraic Delayed DES . . . . . . . . . . . . . . 37

3.3.1 Parallel evaluation of wall-normal line data . . . . . . . . . 38

3.3.2 Algebraic boundary-layer sensors . . . . . . . . . . . 38

3.3.3 Improved LES filter width in the ADDES . . . . . . . . . . . . 42

3.3.4 Synthetic turbulence at the RANS-to-LES interface . . . . . . 43

3.3.5 Sponge layer at the LES-to-RANS interface . . . . . . . . . . . 45

4 Application of the grid-adaptive algebraic hybrid RANS/LES model 47

4.1 Precursor simulations of a three-element airfoil . . . . . . . . . . . . . 49

4.2 Turbulent channel flow . . . . . . . . . . . . . . . . 51 
4.3 Backward-facing step flow $\ldots \ldots \ldots \ldots \ldots \ldots \ldots$

4.4 Wall-mounted hump flow . . . . . . . . . . . . . 58

4.5 Two-element airfoil with undisturbed onflow . . . . . . . . . . . 60

4.6 Transport of a lateral vortex . . . . . . . . . . . . . . . . 68

4.7 Two-element airfoil with vortex interaction . . . . . . . . . . . 69

$\begin{array}{ll}\text { Conclusion } & 75\end{array}$

$\begin{array}{ll}\text { References } & 77\end{array}$

\section{Appendix - Publications}

[A] Chimera technique for transporting disturbances . . . . . . . . . 85

[B] Hybrid RANS/LES simulations of a three-element airfoil . . . . . . . . . 105

[C] Investigation of the Resolution Requirements for a Hybrid RANS/LES Simulation of a Multi-Element Airfoil . . . . . . . . . . . . 117

[D] Assessment of Local LES-Resolution Sensors for Hybrid RANS/LES Simulations . . . . . . . . . . . . . . . . . . 133

[E] Numerical investigation of the DLR F15 two-element airfoil using a Reynolds stress model . . . . . . . . . . . . . . . . . . . . . 145

[F] Hybrid RANS/LES Study of the Development of an Airfoil-Generated Vortex . . . . . . . . . . . . . . . . . . . . 157 


\section{Symbols}

\section{Dimensionless flow parameters}

$\begin{array}{ll}K n & \text { Knudsen number } \\ \mathrm{Ma} & \text { Mach number } \\ \mathrm{Pr} & \text { Prandtl number } \\ \mathrm{Re} & \text { Reynolds number }\end{array}$

\section{$\underline{\text { Tensors }}$}

$\begin{array}{ll}\boldsymbol{I} & \text { Unity matrix } \\ \boldsymbol{P} & \text { Preconditioning matrix } \\ \boldsymbol{S} & \text { Strain-rate tensor } \\ \boldsymbol{\tau} & \text { Viscous stress tensor } \\ \boldsymbol{\tau}_{\text {RANS }}, \boldsymbol{\tau}_{\text {LES }} & \text { Reynolds stress tensor }\end{array}$

\section{Vectors}

$$
\begin{aligned}
& \boldsymbol{x}=(x, y, z)^{T} \\
& \boldsymbol{u}=(u, v, w)^{T} \\
& \boldsymbol{w}=(\rho, \rho \boldsymbol{u}, \rho E)^{T} \\
& \mathcal{R}(\boldsymbol{w}) \\
& \nabla \\
& \boldsymbol{q} \\
& \boldsymbol{\omega}
\end{aligned}
$$

\section{Scalars}

$a$
$d_{w}$
$\delta_{i j}$
$e$
$\epsilon$
$\eta$
$E$
$\gamma$
$\mathcal{G}$
$k$
$k_{\text {tot }}$
$k_{\text {res }}$
$k_{\text {sgs }}$
$k_{\text {kin }}$
$k_{\beta}$
$k^{(4)}$
$\kappa$
$l_{t}$
$\mu$
$\mu_{t}$
$\nu$
$\omega$
$p$

Position vector

Velocity

Conservative variables

Residual of the conservative variables $\boldsymbol{w}$

Vector differential operator

Heat flux vector

Vorticity vector

Speed of sound

Wall distance

Kronecker Symbol

Internal energy

Dissipation rate

Kolmogoroff length scale

Total energy

Ratio of specific heats

Filterning kernel in LES

Turbulent kinetic energy

Total turbulent kinetic energy

Resolved turbulent kinetic energy

Sub-grid turbulent kinetic energy

Total kinetic energy

Cut-off value for preconditioning matrix

Global scaling factor for aritificial dissipation

Heat conductivity, wave number, von Kármán constant

Turbulent length scale

Dynamic molecular viscosity

Eddy viscosity

Kinematic viscosity

Specific dissipation rate

Pressure 


$\begin{array}{ll}\psi_{I J} & \text { Cell-stretching coefficient } \\ \rho & \text { Density } \\ R & \text { Specific gas constant } \\ S & \text { Grid-resolution sensor } \\ t & \text { Time } \\ \tau_{\nu} & \text { Kolmogoroff time scale } \\ T & \text { Temperature } \\ T_{u} & \text { Turbulence intensity } \\ u_{\infty} & \text { Free-stream velocity } \\ u_{\tau} & \text { Skin friction velocity } \\ u_{\nu} & \text { Kolmogoroff velocity scale } \\ u^{+} & \text {Viscous velocity } \\ y^{+} & \text {Viscous length scale }\end{array}$

\section{Model functions and constants}

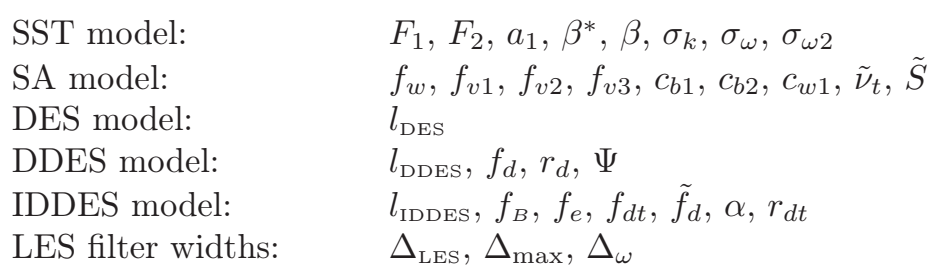

\section{Computational grid}

$\Omega$

$\partial \Omega$

$\mathcal{X}, \widetilde{\mathcal{X}}$

$V_{I}$

$\mathcal{N}^{k}(\boldsymbol{x})$

$\boldsymbol{n}_{I J}$
Computational domain

Boundary of computational domain

Primary grid and dual grid

Control volume in dual grid

Set of $k$-th neighbors of point $\boldsymbol{x}$

Area-weighted face-normal vector between $V_{I}$ and $V_{J}$

\section{Mathematical symbols}

$\begin{array}{ll}\bar{\phi} & \text { Averaged variable } \phi(\mathrm{RANS}), \text { filtered variable } \phi(\mathrm{LES}) \\ \phi^{\prime} & \text { Fluctuating variable } \phi(\mathrm{RANS}), \text { sub-grid scale variable } \phi \\ & \text { (LES) } \\ \langle\phi\rangle & \text { Variable } \phi \text { explicitly averaged in time } \\ \|\phi\| & \text { Euclidean norm of vector } \phi\end{array}$




\section{Abbreviations}

$\begin{array}{ll}\text { ADDES } & \text { Algebraic Delayed Detached-Eddy Simulation } \\ \text { ADT } & \text { Alternating digital tree } \\ \text { DDES } & \text { Delayed Detached-Eddy Simulation } \\ \text { DES } & \text { Detached-Eddy Simulation } \\ \text { DFG } & \text { Deutsche Forschungsgesellschaft (German Research Foundation) } \\ \text { DLR } & \text { Deutsches Zentrum für Luft- und Raumfahrt (German Aerospace } \\ & \text { Center) } \\ \text { DNS } & \text { Direct Numerical Simulation } \\ \text { EVM } & \text { Eddy-viscosity model } \\ \text { FNG } & \text { Flügel neuer Generation (Next generation airfoil) } \\ \text { GIS } & \text { Grid-induced separation } \\ \text { HRLM } & \text { Hybrid RANS/LES model } \\ \text { IDDES } & \text { Improved Delayed Detached Eddy Simulation } \\ \text { JHh } & \text { Jaçirliç-Hanjaliç homogeneous } \\ \text { LES } & \text { Large Eddy Simulation } \\ \text { LLM } & \text { Log-layer mismatch } \\ \text { LU-SGS } & \text { Lower-upper symmetric Gauss-Seidel } \\ \text { PANS } & \text { Partially-averaged Navier-Stokes } \\ \text { PIV } & \text { Particle image velocimetry } \\ \text { RANS } & \text { Reynolds averaged Navier-Stokes } \\ \text { RSM } & \text { Reynolds stress model } \\ \text { SA } & \text { Spalart Allmaras } \\ \text { SAS } & \text { Scale-adaptive simulation } \\ \text { SEM } & \text { Synthetic-eddy method } \\ \text { SST } & \text { Shear-stress transport } \\ \text { STG } & \text { Synthetic turbulence generator } \\ \text { WALE } & \text { Wall-Adapting Local Eddy-viscosity } \\ & \end{array}$





\section{Introduction}

An important driver in the design of modern civil aircrafts is the minimization of fuel consumption through the reduction of aerodynamic drag in the cruise flight. However, a critical factor with respect to safe flying conditions is the aircraft behavior at the border of the flight envelope. As the flow conditions approach the maximum lift of the airplane, measured by the lift coefficient $C_{A \text {,max }}$, incipient flow separation sets in. Beyond $C_{A \text {,max }}$, flow separation leads to a sudden breakdown in lift, called stall, which can have fatal consequences. In the regime of maximum lift, i.e. before stall, the flow is very sensitive to turbulent disturbances. Therefore, to define the safety margins of an airplane, among many other aspects the impact of onflow disturbances on the airplane must be quantified. Nowadays, numerical flow simulations are an integral part of the design process of new aircrafts. To obtain reliable simulation results, the numerical model must be able to represent the relevant physical features of flow separation and the effect of turbulent disturbances. However, the numerical prediction of turbulence in flows at high Reynolds numbers is still a major challenge.

This work contributes to the development of a highly accurate simulation method for the problem of an airplane flying at the border of the flight envelope in disturbed ambient air. The aim of the work is twofold: On the one hand, to develop an improved modeling concept for aerodynamic flows close to and beyond stall in order to enable reliable numerical predictions. On the other hand, to define and investigate a strategy for the representation of a realistic disturbance in a numerical simulation.

Concerning the first point, in general the highest lift force is needed during the takeoff and landing of an airplane. In these phases the reduced air speed is compensated by higher angles of attack and additional high-lift systems, i.e. the slats and flaps are deployed to increase the lift and delay the stall. The numerical prediction of high-lift flows is challenging as the complex flow topology comprises regions of attached flow as well as massive turbulent separation.

There exist many approaches with varying complexity to compute the influence of turbulence. In a Direct Numerical Simulation (DNS), sometimes referred to as numerical experiment, all scales in the turbulent spectrum are resolved. Even though with increasing computing power DNS has become feasible for flows at higher Reynolds numbers, DNS still is rather a tool for turbulence research, as described by Moin and Mahesh [51]. While the detailed results from DNS provide valuable reference data, for most practical applications another way of computing the influence of turbulence is indispensable.

In the Reynolds-Averaged Navier-Stokes (RANS) approaches the impact of the turbulence on the mean flow is approximated in a purely statistical manner, see e.g. Wilcox [89]. The RANS models have reached a level of maturity, which makes them a valuable tool in the design process of aircrafts, see e.g. Abbas-Bayoumi and Becker [2]. But while many RANS models are able to predict attached flows with sufficient accuracy, the results deteriorate considerably for separated flows, see e.g. Rumsey and Ying [66]. During the ongoing work in the RANS model improvement, the focus has recently shifted from Eddy-Viscosity Models (EVM) towards differential Reynolds Stress Models (RSM), which are suited to capture anisotropies of the turbulence and streamline curvatures more accurately. Besides the established EVMs by Spalart and Allmaras [76] and Menter [45], a novel RSM by Cécora et al. [7] is utilized in this work, which is based on the JHh model by Jakirlić and Hanjalić [28] and the JHh-v1 model by Probst et al. [63]. 
Another approach in turbulence modeling is the Large-Eddy Simulation (LES). As in LES only the largest turbulent scales are resolved, while the influence of the smallest scales is approximated through a model, its complexity lies between DNS and RANS. Lesieur and Métais [40] give an overview of the developments in LES. In the present thesis the models by Smagorinsky [73] and the Wall-Adapting Local Eddy-viscosity (WALE) model by Nicoud and Ducros [54] are utilized. One of the strengths of LES is its ability to predict turbulent separated flows more accurately than RANS. On the other hand, the high resolution requirements for the prediction of wall-bounded flows are a major drawback.

To close the gap between RANS and LES, several modeling approaches have been proposed, for example the Scale-Adaptive Simulation (SAS) by Menter et al. [47] and the Partially Averaged Navier-Stokes (PANS) model by Girimaji [22]. Besides, the Detached-Eddy Simulation (DES) by Spalart et al. [77] is a hybrid RANS/LES model, that combines the advantages of both methods: the RANS approach provides reliable results in regions of attached flow, while LES is employed locally to model separated flow regions. Further DES-type models are the Delayed Detached-Eddy Simulation (DDES) by Spalart et al. [78] as well as the Improved Delayed DetachedEddy Simulation (IDDES) by Travin et al. [85].

Spalart [75] gives an overview of the progress in the field of the hybrid RANS/LES approaches. For example, several modifications of the coupling mechanism of RANS and LES have been proposed: Deck [13] employs user-defined regions in the Zonal DES (ZDES) and Shur et al. [72] use overlapping grids, to define concurrent RANS and IDDES zones. But instead of user-defined regions, which require prior knowledge of the flow, an approach is desirable that is able to automatically detect the regions where the model should be modified. This requirement was further promoted through observations by Probst et al. [63], who observe shortcomings of SA-based DDES and IDDES in predicting the trailing edge separation of a single element airfoil, see Sect. 3.1. As a consequence Knopp et al. [35] developed the Algebraic Delayed Detached-Eddy Simulation (ADDES), which is considered in the present thesis. In the ADDES algebraic sensors are used to detect the state of the flow and adjust the model automatically if necessary, as described in Sect. 3.3.2.

In all numerical simulations the grid resolution determines the spatial discretization error, but in scale-resolving simulations the grid-cell size also determines the length scale in the turbulence model. To accurately represent the physical problem in time and space, it is not only necessary to minimize the discretization error, but even more so to sufficiently resolve the larger scales in the turbulent spectrum. Various approaches to assess the grid resolution in an LES have been proposed. For example, Celik et al. [8] and Klein [31] use systematic grid and model variations to distinguish the numerical error from the modeling error. However, for complex test cases it is not affordable to perform exhaustive grid variations. Therefore, the grid-resolution sensor that is proposed in the present thesis is able to assess the grid resolution from a single simulation, see Sect. 3.2.2. Moreover, the resolution sensor can be used as input for a local grid refinement, to increase the resolution if necessary.

One problem, inherent in the DES-type approaches, is the model behavior at the RANS-to-LES transition, the so called grey-area problem. Several strategies for the mitigation of this problem have been proposed. For example, Kok and van der Ven [37] use a high-pass filter to reduce the eddy viscosity in shear layers, while Mockett et al. [49] couple RANS with the $\sigma$ sub-grid model by Nicoud et al. [55], which is sensitized to the orientation and the anisotropy of the resolved field. In the present thesis, the proposal by Chauvet et al. [9] to use a modified length scale in the LES 
region is pursued. To this end, the formulation is adapted to the unstructured grid approach of the TAU code. Another possibility to stimulate the LES model is to induce synthetic turbulent structures, see e.g. the review article by Tabor and BabaAhmadi [83]. Among many other approaches, the Synthetic Turbulence Generator (STG) by Adamian and Travin [3], and the Synthetic-Eddy Method (SEM) by Jarrin et al. [29] were published.

The second focus of the present thesis is the influence of turbulent onflow disturbances on the stall behavior of airfoils. In many publications only simplified model disturbances are considered. Svärd et al. [82] use higher-order methods to study the interaction of an analytical vortex with a NACA-0021 airfoil, while Kim et al. [30] consider an analytical vortex pair interacting with an rigid sphere. Abate [1] prescribes a cosine-shaped gust via an inflow boundary condition. Realistic turbulence is considered by Helmke et al. [26] who obtain highly resolved LES data of an atmospheric boundary layer to assess the general effects of air turbulence on wings. A systematic classification of atmospheric gusts by their shapes and strengths is given by Knigge [33]. Another source of turbulence is the wake of obstacles like preceding aircrafts or buildings near an airport. For example, Leclercq and Doolan [38] investigate the interaction of a vortex wake with a bluff body. In the present thesis a realistic disturbance is generated by a rapidly deflected airfoil which interacts with a two-element airfoil in high-lift configuration. An experimental study of this test case is performed by Hahn et al. [24] and Klein [31], while RANS simulations are conducted by Wawrzinek et al. [87] to investigate the influence of the position of the vortex-generator airfoil.

The own developments that were obtained in the present work, are implemented and applied in the DLR-TAU code, see e.g. Schwamborn et al. [70]. The flow solver TAU is an unstructured finite-volume solver for the compressible Navier-Stokes equations.

This cumulative thesis is organized in four chapters and the appendix. In the first two chapters the theoretical background of the work is given. In Chap. 1 the compressible Navier-Stokes equations and the treatment of turbulence in numerical simulations are described. The flow solver TAU is introduced in Chap. 2. Emphasis is put on the last two chapters where the achievements of the work are presented. Chap. 3 describes implementation details of the new developments in the grid-adaptive Algebraic Delayed Detached-Eddy Simulation model. In Chap. 4 these extended methods are first validated for basic test cases with relevant flow phenomena, before they are used to simulate the target application, i.e. the two-element airfoil with onflow disturbances. The appendix comprises the publications with major own contributions, which were authored during this work.

\section{Overview over Publications}

During the development of the improved simulation strategy presented here, intermediate results were published at several work stages. Other publications were generated in collaboration with co-workers in complementary fields of research. The publications with a major own contribution are attached in the appendix of the present thesis, and they are summarized in the next paragraph. The own contribution to the remaining publications is pointed out in the subsequent paragraph. 


\section{Publications with major own contribution}

[A] S. Reuß, C. Wolf, T. Knopp, A. Raichle, D. Schwamborn: Chimera technique for transporting disturbances. International Journal for Numerical Methods in Fluids, 70(12) pp. 1558-1572, 2010

[B] S. Reuß, T. Knopp, D. Schwamborn: Hybrid RANS/LES simulations of a threeelement airfoil. $\mathrm{Fu}$ et al. (Eds.): Progress in Hybrid RANS-LES Modelling, NNFM 117, pp. 357-367, Springer, 2012

[C] S. Reuß, T. Knopp, D. Schwamborn: Investigation of the Resolution Requirements for a Hybrid RANS/LES Simulation of a Multi-Element Airfoil. Kroll et al. (Eds.): Computational Flight Testing, NNFM 123, pp. 43-57, Springer, 2013

[D] S. Reuß, T. Knopp, A. Probst, M. Orlt: Assessment of Local LES-Resolution Sensors for Hybrid RANS/LES Simulations. Girimaji et al. (Eds.): Progress in Hybrid RANS-LES Modelling, NNFM 130, pp. 93-103, Springer, 2015

[E] S. Reuß, A. Probst, T. Knopp: Numerical investigation of the DLR F15 twoelement airfoil using a Reynolds stress model. In Joint Symposium "Simulation of Wing and Nacelle Stall", Jun. 21-22, 2012, Braunschweig, Germany, 2012

[F] S. Reuß, A. Probst, T. Knopp, K. Wawrzinek: Hybrid RANS/LES Study of the Development of an Airfoil-Generated Vortex. Radespiel et al. (Eds.): Advances in Simulation of Wing and Nacelle Stall, NNFM 131, pp. 41-54, Springer, 2015

The present thesis is the continuation of the work by Wolf [92]. He performed several of the simulations that are presented in Ref. [A], which were already presented in Wolf et al. [93]. Ref. [A] assesses the possibility of transporting vortices, both analytical and airfoil-generated, using the Chimera method. The impact of the grid resolution and the numerical method on the preservation of the vortex is evaluated. Analytical disturbances interact with a NACA-0021 airfoil and an Onera-A airfoil, and a RANS simulation of a realistic airfoil-generated vortex with a high-lift airfoil is performed, the target application of the present thesis.

One aim of the present thesis is to provide an improved hybrid RANS/LES modeling strategy for the reliable prediction of airfoils close to maximum lift. Moreover, a scale-resolving simulation allows to investigate the influence of the various scales of the turbulent onflow disturbance on the airfoil. To start with, in Ref. [B] and Ref. [C] the possibilities and limitations of the original DES-type models are assessed with the help of SA-based DDES and IDDES simulations of the DLR F15 three-element airfoil. Ref. [B] focuses on the comparison of the different modeling approaches, while Ref. [C] addresses the resolution requirements. In particular, the feasibility of a globally scale-resolving approach for complex test cases is scrutinized. None of the applied approaches in Ref. [B] and Ref. [C] were found to capture the trailing-edge separation correctly, which clearly motivated an improved simulation strategy for high-lift flows.

A key element to the reliability of a scale-resolving simulation is the grid resolution: As the grid-cell size is not only coupled to the discretization error, but also influences the model itself, a sufficient resolution is crucial to resolve the relevant physics of the turbulent scales. Therefore, in Ref. [D] a novel grid-resolution sensor is proposed. On the one hand this sensor provides a means to assess the computational grid, but moreover, this sensor can be used as input for a local grid refinement of 
underresolved regions. The sensor is evaluated for the test case of a backward-facing step, and additionally the results of a local grid refinement are presented in Ref. [D]. Furthermore, to mitigate the grey area at the RANS-to-LES interface an improved filter width $\Delta_{\omega}$ is applied in these applications.

For the target application of an airfoil-generated vortex interacting with a high-lift airfoil, the computational costs to simulate the whole experimental wind-tunnel setup are prohibitively high. Therefore, in Ref. [E] a suitable numerical representation of the experimental setup is investigated. Two-dimensional and three-dimensional RANS simulations are compared, and it is concluded that considering only the centerplane section of the wind tunnel yields satisfactory agreement with experimental data.

In Ref. [F] a simulation strategy for the transport of an airfoil-generated disturbance is developed. Relevant characteristics of the generic vortex are identify with the help of the experimental data by Hahn et al. [24]. In the simulations the SST-RANS model as well as the SST-based ADDES are utilized to model the transport of the generic vortex. The comparison with experimental data shows, that the RANS approach is able to predict the mean values of the transported vortex with acceptable accuracy. Therefore, in the target application the vortex transport is treated in RANS mode.

The final results of the target application have not been published. To complete the present thesis, they are presented in Sect. 4.7.

\section{Publications with minor own contribution}

[G] A. Probst, S. Reuß: Scale-Resolving Simulations of Wall-Bounded Flows with an Unstructured Compressible Flow Solver. Girimaji et al. (Eds.): Progress in Hybrid RANS-LES Modelling, NNFM 130, pp. 481-491, Springer, 2015

[H] A. Probst, J. Löwe, S. Reuß, T. Knopp, R. Kessler: Scale-Resolving Simulations with a Low-Dissipation Low-Dispersion Second-Order Scheme for Unstructured Finite-Volume Flow Solvers. AIAA 2015-0816, 2015.

[J] D. G. François, R. Radespiel, S. Reuß, A. Probst: Computations of Separated Flows with Hybrid RANS/LES Approaches. In Radespiel et al. (Eds.): Advances in Simulation of Wing and Nacelle Stall, NNFM 131, Springer, 2015

[K] R. Radespiel, D. G. François, D. Hoppmann, S. Klein, P. Scholz, K. Wawrzinek, T. Lutz, T. Auerswald, J. Bange, C. Knigge, S. Raasch, P. Kelleners, R. Heinrich, S. Reuß, A. Probst, T. Knopp: Simulation of Wing Stall. AIAA 2013-3175, 2013.

For the spatial discretization of the flow equations, the unstructured TAU code applies a standard second-order central scheme, which is stabilized by a matrixvalued artificial dissipation. To improve the results of scale-resolving simulations, this scheme has been optimized in terms of dissipation, see Ref. [G]. In first simulations, the values of various parameters in the artificial-dissipation operator are optimized for wall-resolved LES simulations of a turbulent channel flow. As a part of the present thesis, SA-based IDDES simulations are contributed to the paper, in order to assess the optimized settings for wall-modeled LES. In all presented simulations the results are improved through the optimized settings.

The standard second-order scheme is further improved by Löwe et al. [42]: in the flux evaluation supplementary information about the gradients is included, and the 
additional degree of freedom is used to optimize the dispersion properties of the scheme. The low-dispersion scheme is tested in Ref. $[\mathrm{H}]$ for a turbulent channel flow. Again, in the first simulations the low-dispersion scheme is tested for wall-resolved LES, while the contribution of the wall-modeled LES results, which are obtained with SST-based IDDES, is a part of the present thesis. In these and also in more complex scale-resolving simulations the low-dispersion scheme yields even better results than the low-dissipation scheme.

In the work of François and Radespiel [17] the STG method by Adamian and Travin [3] is improved and implemented in TAU to introduce synthetic turbulent structures at the RANS-to-LES interface. As a part of the present thesis the contribution to Ref. $[\mathrm{J}]$ consists in the extension of the ADDES framework. A data structure is provided, which allows the flexible detection of the reference points for the synthetic turbulence generator. Moreover, this structure enables the addition of synthetic turbulent structures at several locations in the flow.

The present work is embedded in the research group FOR-1066, funded by the Deutsche Forschungsgemeinschaft (DFG - German Research Foundation), which is dedicated to the investigation of wing and nacelle stall. Ref. [K] illustrates the collaboration in this research group, and contributions of the various partners are presented. 


\section{Chapter 1}

\section{Turbulence: physical background and modeling}

In the present thesis the flow of air about a given geometry is considered. The flow conditions include a sufficiently small Knudsen number ${ }^{1}$ to assume a continuous fluid and a free-stream Mach number in the range of $M a=0-0.3$. Even if the effects of compressibility are small in this $M a$ range, locally much higher values can occur in regions of strong flow acceleration, for example, close to the nose of an airfoil. Therefore, the compressible Navier-Stokes equations are considered, which describe the dynamics of a continuous Newtonian fluid. The equations are given in Sect. 1.1. The physical background of the turbulent energy transfer is briefly described in Sect. 1.2. In Sect. 1.3 the concepts of turbulence modeling that are relevant for the present thesis are introduced.

\subsection{Compressible Navier-Stokes equations}

Following from the conservation equations for mass, momentum, and energy, the Navier-Stokes equations describe the fields of density $\rho$, momentum $\rho \boldsymbol{u}=\rho(u, v, w)^{T}$, and total energy $\rho E$ in the spatio-temporal domain $\Omega \times\left(t_{0}, t_{\text {end }}\right)$ with $\Omega \subset \mathbb{R}^{3}$. According to Schlichting and Gersten [68], in the absence of external forces the equations in Cartesian tensor notation ${ }^{2}$ are given by

$$
\begin{aligned}
\frac{\partial \rho}{\partial t}+\frac{\partial\left(\rho u_{i}\right)}{\partial x_{i}} & =0, \\
\frac{\partial\left(\rho u_{i}\right)}{\partial t}+\frac{\partial\left(\rho u_{i} u_{j}\right)}{\partial x_{j}} & =-\frac{\partial p}{\partial x_{i}}+\frac{\partial \tau_{i j}}{\partial x_{j}}, \\
\frac{\partial(\rho E)}{\partial t}+\frac{\partial\left(\rho u_{i} E\right)}{\partial x_{i}} & =-\frac{\partial\left(u_{i} p\right)}{\partial x_{i}}+\frac{\partial\left(u_{i} \tau_{i j}\right)}{\partial x_{j}}+\frac{\partial q_{i}}{\partial x_{i}} .
\end{aligned}
$$

Following Stokes' assumption the viscous stress tensor $\boldsymbol{\tau}$ for Newtonian fluids can be computed from the strain rate tensor $\boldsymbol{S}$. They are component-wise defined as

$$
\tau_{i j}=2 \mu\left(S_{i j}-\delta_{i j} \frac{1}{3} \frac{\partial u_{k}}{\partial x_{k}}\right) \quad \text { and } \quad S_{i j}=\frac{1}{2}\left(\frac{\partial u_{i}}{\partial x_{j}}+\frac{\partial u_{j}}{\partial x_{i}}\right) .
$$

The dynamic molecular viscosity $\mu$ and the temperature $T$ are related through Sutherland's law, which reads

$$
\mu=\mu_{\text {ref }}\left(\frac{T}{T_{\text {ref }}}\right)^{3 / 2} \frac{T_{\text {ref }}+T_{S}}{T+T_{S}} .
$$

\footnotetext{
${ }^{1}$ The Knudsen number $K n$ is given by the ratio of the molecular mean free path to a characteristic physical length.

${ }^{2}$ The Einstein summation convention is used, meaning summation over indices that appear twice in a single term $\frac{\partial u_{i}}{\partial x_{i}}=\frac{\partial u_{1}}{\partial x_{1}}+\frac{\partial u_{2}}{\partial x_{2}}+\frac{\partial u_{3}}{\partial x_{3}}=\nabla \cdot \boldsymbol{u}$. In the following $\boldsymbol{x}=(x, y, z)^{T}$ denotes the vector components, when necessary.
} 
For air the reference temperature $T_{\text {ref }}$, the reference viscosity $\mu_{\text {ref }}$, and the Sutherland temperature $T_{S}$ are given by

$$
T_{\text {ref }}=273.15 \mathrm{~K}, \quad \mu_{\mathrm{ref}}=1.716 \times 10^{-5} \mathrm{~kg} /(\mathrm{ms}), \quad \text { and } \quad T_{S}=110.4 \mathrm{~K} .
$$

For calorically perfect gas the pressure $p$, the temperature $T$, and the specific internal energy $e$ are provided by the equations of state

$$
p=\rho R T \quad \text { and } \quad e=\frac{1}{1-\gamma} R T,
$$

where $R=287.058 \mathrm{~J} /(\mathrm{kgK})$ is the specific gas constant and $\gamma=1.4$ is the ratio of specific heats of air. The total energy $E$ is the sum of the internal energy $e$ and the kinetic energy $k_{\text {kin }}$ with

$$
E=e+k \quad \text { and } \quad k_{\text {kin }}=\frac{1}{2} u_{i} u_{i} .
$$

By Fourier's law the heat flux vector $\boldsymbol{q}$ is proportional to the temperature gradient

$$
q_{i}=\kappa \frac{\partial T}{\partial x_{i}} .
$$

The thermal conductivity $\kappa$ can be computed from the Prandtl number, which is $\operatorname{Pr}=0.72$ for air. It is defined as the ratio of the viscous diffusion to the thermal diffusion

$$
\operatorname{Pr}=\frac{\frac{\mu}{\rho}}{\frac{\kappa}{\rho C_{p}}}=\frac{\gamma}{\gamma-1} R \frac{\mu}{\kappa} .
$$

For $\phi \in\{\rho, \rho \boldsymbol{u}, \rho E\}$ the terms in Eqs. (1.1)-(1.3) can be characterized as the transient term $\frac{\partial \phi}{\partial t}$ and the convective term $\frac{\partial\left(u_{i} \phi\right)}{\partial x_{i}}$ on the left hand side; the right hand side contains the pressure terms with $p$, the viscous terms with $\boldsymbol{\tau}$, and the heat flux $\boldsymbol{q}$.

To solve the problem (1.1)-(1.3), initial values at $t=t_{0}$ and boundary values ${ }^{3}$ at the borders of the computational domain $\partial \Omega$ are given by

$$
\begin{array}{ll}
\phi\left(t_{0}, \boldsymbol{x}\right)=\phi_{0}, & \forall \boldsymbol{x} \in \Omega, \\
\left.\phi(t, \boldsymbol{x})\right|_{\partial \Omega}=B \phi, & \forall \boldsymbol{t} \in\left(t_{0}, t_{\text {end }}\right), \text { with the boundary operator } B .
\end{array}
$$

The state of a viscous compressible fluid flow can generally and uniquely be described by the characteristic dimensionless Reynolds number Re and the Mach number Ma. The Reynolds number is determined by the ratio

$$
\frac{\text { convective term }}{\text { viscous term }}=\frac{u \frac{\partial u}{\partial x}}{u \frac{\partial^{2} u}{\partial x^{2}}} \approx \frac{U \frac{U}{L}}{\nu \frac{U}{L^{2}}}=\frac{U L}{\nu}=R e,
$$

with the kinematic viscosity $\nu$, the characteristic velocity $U$, and the characteristic length scale $L$. In the present thesis, $U$ is usually the free-stream velocity $u_{\infty}$, and $L$ can be the wing chord or the channel height, for example. The Mach number, defined as the ratio of the characteristic flow velocity to the speed of sound $a$, is given by

$$
M a=\frac{U}{a} \quad \text { with } \quad a=\sqrt{\gamma R T} .
$$

\footnotetext{
${ }^{3}$ The boundary conditions are constant in time for all simulations in the present thesis.
} 


\subsection{Description of turbulence}

At very low Reynolds numbers the flow is usually laminar, which means that layers of fluid with different velocities slide over each other in a parallel flow, and diffusive transport is the only mechanism that causes mixing between the layers. With increasing Reynolds number the influence of the non-linear convective term grows and beyond a critical Reynolds number the flow becomes turbulent. The turbulent motion of the fluid leads to additional mixing of the flow. The change from a laminar to a turbulent flow state is called the laminar-turbulent transition, but this process, which is a field of research on its own, is not a subject of the present thesis ${ }^{4}$.

In turbulent flows vortical structures exist, called eddies, which interact with each other. The length scales and time scales associated with the structures cover a broad range. In flows that are in turbulent equilibrium, the interaction of the different turbulent length scales $l_{t}$ can be described by the energy spectrum, see e.g. Pope [60].

In Fig. 1.1 the energy spectrum function $E(\kappa)$ is plotted over the wave number $\kappa=\frac{2 \pi}{l_{t}}$, where the turbulent kinetic energy contained in the wave numbers $\kappa \in\left(\kappa_{a}, \kappa_{b}\right)$ is given by

$$
k\left(\kappa_{a}, \kappa_{b}\right)=\int_{\kappa_{a}}^{\kappa_{b}} E(\kappa) \mathrm{d} \kappa .
$$

The spectrum illustrates that the larger turbulent structures, as they break up into

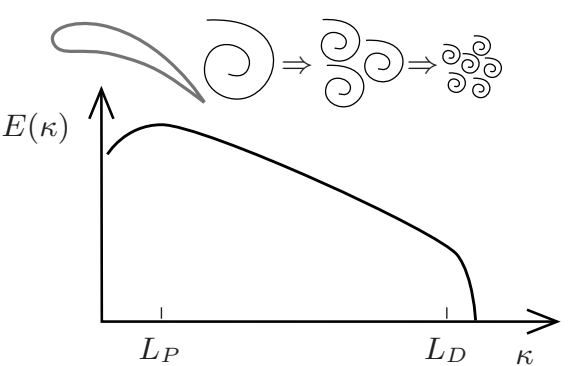

Fig. 1.1: The turbulent energy spectrum. smaller structures, transfer their turbulent kinetic energy in a cascading process. The energy spectrum can be divided into three regions: the production region $l_{t} \approx L_{P}$, the transfer region $L_{P}>l_{t}>L_{D}$, and the dissipation region $l_{t} \leq L_{D}$. In the production region the size of the largest vortices is determined by the dimension and geometry of the flow problem, for example, by the diameter of a channel or the chord length of an airfoil. The largest vortices receive energy from the mean flow through the production mechanism, and they are typically anisotropic by generation. At large Reynolds numbers the effect of dissipation on the vortices in the production region can be neglected.

In the transfer region the vortices receive energy only from larger vortices by inviscid interaction. The geometry-generated anisotropy of the vortices reduces, and on average they exhibit an universal behavior, statistically independent of the largest scales, which is described by Tennekes and Lumley [84].

In the dissipation range the turbulent kinetic energy of the smallest vortices is converted into thermal energy. Kolmogoroff's universal equilibrium theory postulates that the rate of energy dissipation is equal to the rate of energy supply of the larger vortices in the transfer region and to the rate at which the largest eddies receive energy from the mean flow. The dissipative scales are independent of the dimension or geometry of the flow, and their universal isotropic shape depends only on the dissipation rate $\varepsilon$ and the kinematic viscosity $\nu$ of the fluid, see e.g. Pope [60]. Based on these quantities, the Kolmogoroff micro scales of length $\eta$, velocity $u_{\nu}$, and time

\footnotetext{
${ }^{4}$ In some of the applications laminar regions are prescribed, which means that the production term of the turbulence model is switched off.
} 
$\tau_{\nu}$ are defined as

$$
\eta=\left(\frac{\nu^{3}}{\varepsilon}\right)^{\frac{1}{4}}, \quad u_{\nu}=(\varepsilon \nu)^{\frac{1}{4}}, \quad \text { and } \quad \tau_{\nu}=\left(\frac{\nu}{\varepsilon}\right)^{\frac{1}{2}} .
$$

Only at sufficiently high Reynolds numbers, the largest scales, which bear the mean part of the turbulent kinetic energy, and the dissipative scales are clearly separated.

\subsection{Numerical simulation of turbulent flows}

A numerical solution of the discretized Navier-Stokes equations can be obtained with an appropriate algorithm in a Direct Numerical Simulation (DNS). For example, Schlatter et al. [67] and Lee and Moser [39] simulate turbulent channel flow using spectral methods, which are very efficient for geometrically simple domains and solutions without strong discontinuities. However, the applicability of DNS is limited because the disparity between the smallest and the largest scales of turbulence increases with the Reynolds number. To resolve the smallest scales, the spacing of the grid cells must be of the same order of magnitude as the Kolmogoroff length scale. Considering this requirement, Choi and Moin [10] estimate the required number of points for a DNS of a flat-plate boundary layer with the Reynolds number Re based on the stream-wise length at

$$
N_{\mathrm{DNS}} \approx R e^{37 / 14} .
$$

The Reynolds numbers of aerodynamic flows about civil aircrafts are typically prohibitively high for a DNS. As an example, the Reynolds numbers for different flight conditions of an Airbus A320 and an A380 are given in Tab. 1.1.

\begin{tabular}{rrrrr}
\hline & Wing span & MAC $^{\dagger}$ & $R e$ take-off & ${\text { Re } \text { cruise }^{\ddagger \dagger}}^{\ddagger \dagger}$ \\
\hline \hline A320 & $34.9 \mathrm{~m}$ & $4.2 \mathrm{~m}$ & $7.5 \times 10^{6}$ & $27.9 \times 10^{6}$ \\
\hline A380 & $79.75 \mathrm{~m}$ & $12.3 \mathrm{~m}$ & $22.0 \times 10^{6}$ & $81.8 \times 10^{6}$ \\
\hline
\end{tabular}

$\dagger$ mean aerodynamic chord

$\ddagger$ take-off: altitude $300 \mathrm{~m}$; airspeed $70 \mathrm{~m} / \mathrm{s}$

${ }^{\dagger \dagger}$ cruise flight: altitude $11,000 \mathrm{~m}$; airspeed $260 \mathrm{~m} / \mathrm{s}$

Tab. 1.1: Reynolds numbers for different flight conditions of an Airbus A320 and an A380.

The two- and three-element airfoils with deployed high-lift devices that are considered in the present thesis correspond to a cut through the wing of an airplane during take-off. However, the Reynolds numbers reflect the conditions in the wind tunnel rather than the realistic free-flight configuration. Besides the Reynolds number, the high ratio of the wing span to the chord length is another aspect: the grid-point requirement in Eq. (1.16), estimated for a developing boundary layer, is needed over the full span of the wing.

In order to treat flows at high Reynolds numbers in a numerical simulation, the necessity to resolve all turbulent length scales in a DNS has to be relieved. The idea is to achieve this by empirically modeling at least parts of the influence of the turbulent motion on the mean flow field. 


\subsubsection{Statistical turbulence modeling - RANS}

The Reynolds-Averaged Navier-Stokes (RANS) equations describe the mean flow field, while the influence of the turbulence on the mean flow is given by an appropriate RANS turbulence model. The resolution in a RANS simulation is therefore no longer determined by the turbulent length scales, but it must be sufficient to represent the gradients of the mean flow.

The idea of Reynolds-averaging is to decompose the variables $\phi$ into a mean part $\bar{\phi}$ and a fluctuating part $\phi^{\prime}$, with

$$
\bar{\phi}(\boldsymbol{x}, t)=\lim _{t_{1} \rightarrow \infty} \int_{t_{0}}^{t_{0}+t_{1}} \phi\left(\boldsymbol{x}, t^{\prime}\right) \mathrm{d} t^{\prime} .
$$

Formally, the RANS equations are obtained by substituting $\phi=\bar{\phi}+\phi^{\prime}$ and taking the average of both sides of the equation, utilizing the following properties of the averaging operator

$$
\overline{\bar{\phi}}=\bar{\phi}, \quad \overline{\phi^{\prime}}=0, \quad \text { and } \quad \overline{\frac{\partial}{\partial x} \phi}=\frac{\partial}{\partial x} \bar{\phi} .
$$

The non-linear convective term in the momentum equations leads to a new term, which contains the second-order correlations of the velocity fluctuations, given by

$$
\overline{\left(\bar{u}_{i}+u_{i}^{\prime}\right)\left(\bar{u}_{j}+u_{j}^{\prime}\right)}=\overline{\bar{u}_{i} \bar{u}_{j}}+\overline{\bar{u}_{i} u_{j}^{\prime}}+\overline{\bar{u}_{j} u_{i}^{\prime}}+\overline{u_{i}^{\prime} u_{j}^{\prime}}=\bar{u}_{i} \bar{u}_{j}+\overline{u_{i}^{\prime} u_{j}^{\prime}}
$$

With this, the Reynolds-averaged momentum equation reads

$$
\frac{\partial}{\partial t}\left(\rho \bar{u}_{i}\right)+\frac{\partial}{\partial x_{j}}\left(\rho \bar{u}_{j} \bar{u}_{i}\right)=-\frac{\partial \bar{p}}{\partial x_{i}}+\frac{\partial}{\partial x_{j}}\left(\bar{\tau}_{i j}\right)-\frac{\partial}{\partial x_{j}} \tau_{\mathrm{RANS}, i j},
$$

where the components of the Reynolds stress tensor $\tau_{\mathrm{RANS}, i j}=\rho \overline{u_{i}^{\prime} u_{j}^{\prime}}$ describe the mean influence of the velocity fluctuations on the mean flow. Since the Reynolds stress tensor $\boldsymbol{\tau}_{\text {RANS }}$ is symmetric, six independent unknown terms are introduced. To close the system of equations, a large variety of turbulence models is available, which describe the unknown terms.

\section{Eddy viscosity modeling}

The class of eddy viscosity models is based on the Boussinesq hypothesis. In analogy to Eq. (1.4), the Reynolds stresses are related to the mean strain rate tensor $\boldsymbol{S}$ with the eddy viscosity $\mu_{t}$ as proportionality factor, by

$$
\rho \overline{u_{i}^{\prime} u_{j}^{\prime}}=2 \mu_{t} \bar{S}_{i j}-\frac{2}{3} \rho k \delta_{i j} \quad \text { with } \quad k=\frac{1}{2} \overline{u_{i}^{\prime} u_{i}^{\prime}}
$$

where $k$ is the turbulent kinetic energy, i.e. the kinetic energy of the turbulent fluctuations. With this approach the eddy viscosity essentially increases the "effective" viscosity of the fluid. Using the turbulent velocity scale $\sqrt{k}$, the turbulent length scale $l_{t}$, or the turbulent time scale $t_{t} \propto 1 / \omega \propto k / \varepsilon$, where $\varepsilon$ is the dissipation rate of the turbulent kinetic energy and $\omega=\frac{\varepsilon}{k}$ is the specific dissipation rate, the eddy viscosity can be expressed as

$$
\mu_{t} \propto \rho \sqrt{k} l_{t} \quad \text { or } \quad \mu_{t} \propto \rho l_{t}^{2} t_{t}^{-1} .
$$


The class of eddy viscosity turbulence models offers a large number of approaches, which use either one or two additional transport equations to determine $\mu_{t}$.

The Shear-Stress Transport two-equation $k-\omega$ model (SST) by Menter [45], with a slightly modified newer version by Menter et al. [48], is constructed by blending the $k$ - $\omega$ model in the inner part of the boundary layer and the $k-\varepsilon$ model in the outer part of the flow to combine the advantages of both: the $k-\varepsilon$ model is insensitive to the prescribed free-stream values, while the $k-\omega$ model is considered more accurate in boundary layers with an adverse pressure gradient. To arrive at a common formulation, the $\varepsilon$-equation is transformed into an $\omega$-equation, where through the transformation an additional cross-diffusion term is introduced. The transport equations for $k$ and $\omega$ are given by

$$
\begin{aligned}
\frac{\partial}{\partial t}(\rho k)+\frac{\partial}{\partial x_{j}}\left(\rho u_{j} k\right) & =\rho \tau_{i j} \frac{\partial u_{i}}{\partial x_{j}}-\beta^{*} \rho k \omega+\frac{\partial}{\partial x_{j}}\left(\left(\mu+\mu_{t} \sigma_{k}\right) \frac{\partial k}{\partial x_{j}}\right) \\
\frac{\partial}{\partial t}(\rho \omega)+\frac{\partial}{\partial x_{j}}\left(\rho u_{j} \omega\right) & =\frac{\gamma \rho}{\mu_{t}} \tau_{i j} \frac{\partial u_{i}}{\partial x_{j}}-\beta \rho \omega^{2}+\frac{\partial}{\partial x_{j}}\left(\left(\mu+\mu_{t} \sigma_{\omega}\right) \frac{\partial \omega}{\partial x_{j}}\right) \\
& +2 \sigma_{\omega 2}\left(1-F_{1}\right) \frac{\rho}{\omega} \frac{\partial k}{\partial x_{j}} \frac{\partial \omega}{\partial x_{j}} .
\end{aligned}
$$

The SST model coefficients are obtained by blending the coefficients $\phi_{1}$ of the $k-\omega$ model close to the wall and the coefficients $\phi_{2}$ of the $k-\varepsilon$ model away from the wall through $\phi=F_{1} \phi_{1}+\left(1-F_{1}\right) \phi_{2}$. The empirical blending function $F_{1}$ takes values close to one in the near-wall region and logarithmic region of the boundary layer. In the wake region the values approach zero. This behavior of $F_{1}$ also deactivates the cross-diffusion term in the $\omega$-equation near the wall. The eddy viscosity is defined as

$$
\mu_{t}=\min \left(\frac{\rho k}{\omega}, \frac{a_{1} \rho k}{\|\boldsymbol{\omega}\| F_{2}}\right) .
$$

The second term in Eq. (1.25), containing the magnitude of the vorticity vector $\boldsymbol{\omega}=\boldsymbol{\nabla} \times \boldsymbol{u}$, represents the SST correction, which prevents the tendency of the twoequation models to overestimate the shear stress in boundary layers with an adverse pressure gradient. Following Bradshaw's assumption the shear stress is proportional to the turbulent kinetic energy in the boundary layer. The SST correction ensures the compliance of this assumption by limiting the stress-intensity ratio to $\overline{u_{i}^{\prime} u_{j}^{\prime}} / k \leq a_{1}$ with the Bradshaw constant $a_{1}=0.31$. The blending function $F_{2}$, which takes a value of 1 inside the boundary layer and goes to zero away from the wall, is introduced to deactivate the SST correction in free shear layers.

In the Spalart-Allmaras one-equation model (SA) by Spalart and Allmaras [76] the turbulent kinetic energy in the Reynolds stress tensor, Eq. (1.21), is neglected. Instead, a transport equation for the modified kinematic eddy viscosity $\tilde{\nu}_{t}$ is formulated as

$$
\begin{aligned}
\frac{\partial \rho \tilde{\nu}_{t}}{\partial t}+\frac{\partial}{\partial x_{j}}\left(\rho \tilde{\nu}_{t} u_{j}\right)=c_{b 1} \rho \tilde{S} \tilde{\nu}_{t} & +\frac{\partial}{\partial x_{j}}\left(\frac{\rho \nu+\rho \tilde{\nu}_{t}}{\sigma} \frac{\partial \tilde{\nu}_{t}}{\partial x_{j}}\right) \\
& +\rho \frac{c_{b 2}}{\sigma}\left(\nabla \tilde{\nu}_{t}\right)^{2}-c_{w 1} f_{w} \rho\left(\frac{\tilde{\nu}_{t}}{d_{w}}\right)^{2} .
\end{aligned}
$$

The terms on the right-hand side represent production, gradient diffusion and wall destruction of $\tilde{\nu}_{t}$, respectively. The function $f_{w}$ in the destruction term is designed to obtain a fast decaying behavior in the outer region of the boundary layer. The 
turbulent length scale is given by the wall distance $d_{w}$. With the von Kármán constant $\kappa=0.41$, the modified vorticity magnitude $\tilde{S}$ in the production term is defined as

$$
\tilde{S}=f_{v 3}\|\boldsymbol{\omega}\|+\frac{\tilde{\nu}_{t}}{\kappa^{2} d_{w}^{2}} f_{v 2} .
$$

The eddy viscosity $\mu_{t}$ is obtained from $\tilde{\nu}_{t}$ through

$$
\mu_{t}=f_{v 1} \rho \tilde{\nu}_{t},
$$

where the damping function $f_{v 1}$ is designed to ensure that $\nu_{t} \approx \kappa u_{\tau} d_{w}$ in the log layer, with the friction velocity $u_{\tau}$ defined as

$$
u_{\tau}=\sqrt{\frac{\tau_{w}}{\rho}} \quad \text { and } \quad \tau_{w}=\left.\nu \frac{\partial u}{\partial y}\right|_{d_{w}=0} .
$$

\section{Differential Reynolds stress modeling}

In the class of differential Reynolds Stress Models (RSM), six transport equations for the Reynolds stresses are provided together with an additional length-scale equation to close the system. There are approaches that use the dissipation of the turbulent kinetic energy $\varepsilon$, the specific dissipation rate $\omega$, or combinations of $\varepsilon$ and $\omega$ to determine the turbulent length scale.

According to Wilcox [89], the transport equations for the Reynolds stresses are given by

$$
\frac{\partial}{\partial t}\left(\rho \overline{u_{i}^{\prime} u_{j}^{\prime}}\right)+\frac{\partial}{\partial x_{k}}\left(\bar{u}_{k} \rho \cdot \overline{u_{i}^{\prime} u_{j}^{\prime}}\right)=\rho P_{i j}+\rho \phi_{i j}+\rho \varepsilon_{i j}+\rho D_{i j} .
$$

The terms on the right-hand side of the equation contain the production tensor $\boldsymbol{P}$, the pressure-strain correlation tensor $\phi$, the dissipation-rate tensor $\varepsilon$, and the diffusion tensor $\boldsymbol{D}$. All components of the tensor $\boldsymbol{P}$ are known, whereas most other terms in Eq. (1.30) have to be modeled appropriately.

In Sect. 4.5 a variant of the JHh RSM model by Jakirlić and Hanjalić [28] is used that utilizes a transport equation for the homogeneous part of the dissipation rate $\varepsilon^{h}$ : the JHh-v2 RSM by Cécora et al. [7]. The derivation of all modeled terms is described in detail by Probst [61].

\subsubsection{Large-eddy simulation - LES}

In a Large-Eddy Simulation (LES) the large turbulent scales are resolved, and only the influence of the smallest scales is modeled. The number of points to resolve a turbulent boundary layer down to the wall with an LES reduces considerably in comparison to the estimation for DNS in Eq. (1.16). Choi and Moin [10] estimate the required number of points for a wall-resolved LES at

$$
N_{\mathrm{LES}} \approx R e^{13 / 7} .
$$

Similar to the RANS approach, in LES the variables are decomposed into $\phi=\bar{\phi}+\phi^{\prime}$, where $\bar{\phi}$ denotes a filtering operation, and $\phi^{\prime}$ denotes the scales that are filtered out. 
Unlike the time averaging in Eq. (1.17), the filtering in LES is an operation in space. The filtered turbulent field $\phi$ can be defined by the convolution integral

$$
\bar{\phi}(\boldsymbol{x}, t)=\int_{\Omega} \mathcal{G}\left(\boldsymbol{x}-\boldsymbol{x}^{\prime}\right) \phi(\boldsymbol{x}, t) \mathrm{d} \boldsymbol{x}^{\prime},
$$

where the filtering kernel $\mathcal{G}$ has a compact support with

$$
\int_{\Omega} \mathcal{G}\left(\boldsymbol{x}-\boldsymbol{x}^{\prime}\right) \mathrm{d} \boldsymbol{x}^{\prime}=1 .
$$

With this definition the properties of the averaging operation in Eq. (1.18) do not hold in general for the filtering operation, instead

$$
\overline{\bar{\phi}} \neq \bar{\phi} \quad \text { and } \quad \overline{\phi^{\prime}} \neq 0 \text {. }
$$

Therefore, compared to Eq. (1.19), in the filtered momentum equation additional terms arise from the non-linear term

$$
\overline{\left(\bar{u}_{i}+u_{i}^{\prime}\right)\left(\bar{u}_{j}+u_{j}^{\prime}\right)}=\overline{\bar{u}_{i} \bar{u}_{j}}+\overline{\bar{u}_{i} u_{j}^{\prime}}+\overline{\bar{u}_{j} u_{i}^{\prime}}+\overline{u_{i}^{\prime} u_{j}^{\prime}} .
$$

To simplify the derivation of the filtered equations, it is assumed that the differentiation and the filtering operator commute. In general this is not the case, for example in boundary layers with insufficient grid-resolution, but the error is neglected. The first term on the right hand side is a part of the Leonard stress tensor $\boldsymbol{L}$, the second and third term build the cross stress tensor $\boldsymbol{C}$, and the fourth term represents the sub-grid stress tensor $\boldsymbol{R}$, given by

$$
L_{i j}=\overline{\bar{u}_{i} \bar{u}_{j}}-\bar{u}_{i} \bar{u}_{j}, \quad C_{i j}=\overline{\bar{u}_{i} u_{j}^{\prime}}+\overline{\bar{u}_{j} u_{i}^{\prime}}, \quad \text { and } \quad R_{i j}=\overline{u_{i}^{\prime} u_{j}^{\prime}} .
$$

In contrast to the RANS Reynolds stress tensor $\tau_{\text {RANS }}$, which contains information about the whole spectrum of turbulent fluctuations, $\boldsymbol{R}$ represents only the small scale structures. According to Piomelli and Chasnov [58], it is common practice to include $\boldsymbol{L}$ and $\boldsymbol{C}$ in the model for the sub-grid scale Reynolds stress tensor

$$
\tau_{\mathrm{LES}, i j}=\rho\left(L_{i j}+C_{i j}+R_{i j}\right),
$$

since neither the Leonard stresses nor the cross stresses are invariant with respect to a Galilean transformation. With this approach, the filtered momentum equation takes the same form as the RANS momentum equation (1.20)

$$
\frac{\partial}{\partial t}\left(\rho \bar{u}_{i}\right)+\frac{\partial}{\partial x_{j}}\left(\rho \bar{u}_{j} \bar{u}_{i}\right)=-\frac{\partial \bar{p}}{\partial x_{i}}+\frac{\partial}{\partial x_{j}}\left(\bar{\tau}_{i j}\right)-\frac{\partial}{\partial x_{j}} \tau_{\mathrm{LES}, i j} .
$$

In the finite-volume discretization the discrete value of $\phi$ in the grid-cell volume $V$ is given by the cell average

$$
\phi(\boldsymbol{x}, t)=\frac{1}{|V|} \int_{V} \phi\left(\boldsymbol{x}^{\prime}, t\right) \mathrm{d} \boldsymbol{x}^{\prime} \quad \text { with } \quad|V|=\int_{V} \mathrm{~d} \boldsymbol{x}^{\prime} .
$$

This formulation corresponds to Eq. (1.32) with an appropriate filter $\mathcal{G}$ that vanishes outside $V$. Therefore, in practical applications the explicit filtering is replaced by implicit filtering through the finite-volume discretization. With this approach the LES length scale is given by

$$
l_{\mathrm{LES}}=C_{\mathrm{LES}} \Delta_{\mathrm{LES}},
$$

with a general model constant $C_{\mathrm{LES}}$, where the LES filter width $\Delta_{\mathrm{LES}}$ is proportional to the grid-cell size. There are several definitions for $\Delta_{\mathrm{LES}}$, which are discussed in Sect. 3.3.3. 


\section{Sub-grid scale modeling}

The influence of the unresolved sub-grid fluctuations $\phi^{\prime}$, represented by $\boldsymbol{\tau}_{\mathrm{LES}}$, is taken into account by the LES sub-grid model. In Sect. 2.3 the Smagorinsky model and the Wall-Adapting Local Eddy-viscosity (WALE) model are applied. Both use the Boussinesq approximation, Eq. (1.21), to relate the unknown sub-grid Reynolds stress $\tau_{\text {LES }}$ to the mean shear stress via the sub-grid eddy viscosity $\mu_{t}$. As the reciprocal magnitude of the strain-rate tensor can be regarded as the time scale of the resolved structures, the eddy viscosity of the sub-grid scales relates $\mu_{t} \propto l_{t}^{2}\|\overline{\boldsymbol{S}}\|$, with $l_{t}$ given by Eq. (1.40).

Near solid walls the turbulent structures are damped, so that $u_{i}^{\prime}=0$ and consequently $\mu_{t}=0$ close to the wall. In the Smagorinsky model this behavior is ensured by van-Driest-type damping functions, such as the one by Piomelli et al. [59], which reads

$$
f_{\mathrm{vD}}=\sqrt{1-e^{\left(-y^{+} / 25\right)^{3}}} \quad \text { with } \quad y^{+}=\frac{u_{\tau} d_{w}}{\nu} .
$$

Here, $y^{+}$is the non-dimensional wall distance, which is normalized using the friction velocity $u_{\tau}$, according to Eq. (1.29). The eddy viscosity in the Smagorinsky model is given by

$$
\mu_{t}=\rho\left(f_{\mathrm{vD}} \cdot C_{\mathrm{smag}} \Delta\right)^{2} \cdot\|\overline{\boldsymbol{S}}\| .
$$

The WALE model by Ducros et al. [15] uses a more complex expression for the turbulent time scale, which automatically fulfills the asymptotic near-wall requirements for $\mu_{t}$ such that no additional damping is necessary. The eddy viscosity in the WALE model is given by

$$
\begin{aligned}
& \mu_{t}=\rho\left(C_{\mathrm{WALE}} \Delta\right)^{2} \cdot \frac{\left(S_{i j}^{d} S_{i j}^{d}\right)^{3 / 2}}{\left(\bar{S}_{i j} \bar{S}_{i j}\right)^{5 / 2}+\left(S_{i j}^{d} S_{i j}^{d}\right)^{5 / 4}}, \\
& \text { with } \quad S_{i j}^{d}=\frac{1}{2}\left(\bar{g}_{i j}^{2}+\bar{g}_{j i}^{2}\right)-\frac{1}{3} \delta_{i j} \bar{g}_{k k}^{2} \quad \text { and } \quad \bar{g}_{i j}=\frac{\partial \bar{u}_{i}}{\partial x_{j}} .
\end{aligned}
$$

\subsubsection{Hybrid RANS/LES modeling}

The idea behind hybrid RANS/LES models is to devise a simulation method that combines the relatively low computational costs of RANS approaches for the prediction of attached turbulent boundary layer flows with the high-fidelity predictive capabilities of LES for massively separated flows. There is a large variety of methods to bring together a RANS model and an LES model in one simulation. Fröhlich and von Terzi [20] identify two main classes: the segregated approaches and the unified approaches.

In the segregated approaches the computational domain is strictly divided into RANS zones $\Omega_{\text {RANS }}$ and LES zones $\Omega_{\text {LES }}$. Usually one or more small LES zones are embedded into one large RANS zone in order to resolve the regions of interest. In $\Omega_{\text {RANS }}$ the averaged Eq. (1.20) are solved for the averaged variables $\bar{\phi}_{\mathrm{RANS}}$, while in $\Omega_{\mathrm{LES}}$ the filtered Eq. (1.38) are solved for the filtered variables $\bar{\phi}_{\mathrm{LES}}$. At interfaces where the flow enters an LES region synthetic turbulent fluctuations are added to the averaged variables, whereas at interfaces where the flow enters a RANS region averaged values 
of the filtered variables are prescribed. For example, sponge terms can be used to damp out the resolved turbulent structures. The inflow variables are denoted as

$$
\bar{\phi}_{\mathrm{LES}, \text { inflow }}=\bar{\phi}_{\mathrm{RANS}}+\phi_{\mathrm{synth}}^{\prime} \quad \text { and } \quad \bar{\phi}_{\mathrm{RANS}, \text { inflow }}=\left\langle\bar{\phi}_{\mathrm{LES}}\right\rangle
$$

The unified approaches are based on the formal equality of the averaged Eq. (1.20) and the filtered Eq. (1.38). The hybrid turbulence model equations are formulated in a manner that allows them to convert from a RANS model to an LES model. The computed variables are continuous and their interpretation as filtered or averaged values depends on the behavior of the model. A subgroup of the unified models are the non-zonal methods, in which the allocation of RANS and LES regions is not fixed by the user, but rather by the model itself. The present thesis is focused on a family of models from this group: the Detached-Eddy Simulation (DES) and variants of it.

To obtain the desired model behavior, the non-zonal models can be manipulated to adopt the RANS or the LES mode in user-specified zones, disregarding the automatic assignment of RANS and LES regions. Besides this simple enforcement of userprescribed zones, Deck [13] summarizes numerous developments under the term Zonal DES (ZDES). However, as with the embedded approaches, the definition of the LES zones requires prior knowledge of the separated regions. This is not trivial in some cases, for example, for separation over a smooth surface, which is driven by an adverse pressure gradient. A remedy for this difficulty can be the algebraic hybrid RANS/ LES model, which is described in Sect. 3.3.

\section{Original Detached-Eddy Simulation - DES}

The original Detached-Eddy Simulation (DES) by Spalart et al. [77] was proposed as a hybrid RANS/LES model for local scale-resolving simulations of massively separated flows. In the RANS mode the turbulent length scale $l_{\text {RANS }}$ is determined by the RANS model, whereas in the LES mode the length scale is given by Eq. (1.40), where the constant is calibrated individually for each turbulence model. ${ }^{5}$ The idea is to turn an existing RANS model into a hybrid RANS/LES model by replacing $l_{\text {RANS }}$ with

$$
l_{\mathrm{DES}}=\min \left(l_{\mathrm{RANS}}, l_{\mathrm{LES}}\right) .
$$

To obtain the SA-based DES model, the occurrences of the wall distance $d_{w}$ in the wall destruction term in Eq. (1.26) and in the modified vorticity magnitude $\tilde{S}$ in Eq. (1.27) are replaced with $l_{\mathrm{DES}}$, with the DES constant $C_{\mathrm{DES}}=0.65$.

In the SST-based DES formulation, $l_{\mathrm{DES}}$ is introduced only in the dissipative term of the $k$-equation (1.23) to keep the modifications as simple as possible, according to Strelets [81]. Reformulated in terms of the RANS length scale, which is then replaced with $l_{\mathrm{DES}}$, the dissipative term reads

$$
\rho \beta^{*} k \omega=\rho \frac{k^{\frac{3}{2}}}{l_{\mathrm{RANS}}} \quad \text { with } \quad l_{\mathrm{RANS}}=\frac{\sqrt{k}}{\beta^{*} \omega} .
$$

For the SST-based DES model the two values $C_{\mathrm{DES}, 1}=0.78$ and $C_{\mathrm{DES}, 2}=0.61$ are blended using the model function $F_{1}$.

Near viscous walls, where $l_{\text {RANS }}<l_{\text {LES }}$, the DES model works in the RANS mode, whereas in regions of massively separated flow the detached eddies are resolved by an LES like sub-grid model. This situation is illustrated in Fig. 1.2.

\footnotetext{
${ }^{5}$ The calibration is usually based on the decay rate and the spectrum of decaying isotropic turbulence.
} 


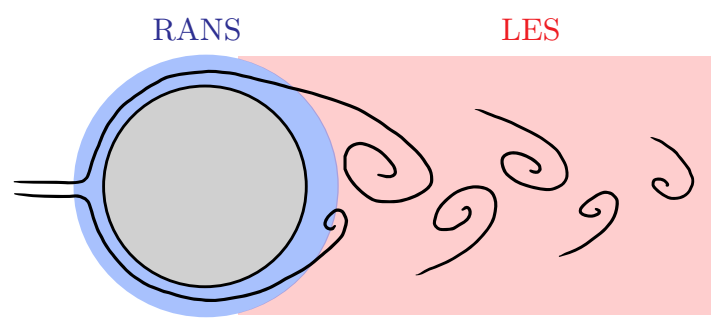

Fig. 1.2: RANS and LES regions in a DES simulation of the flow about a cylinder.

By construction, the location where the original DES switches from RANS to LES depends solely on the grid-cell size, while the actual flow behavior is not considered in the model. If the grid spacing near the wall is small, it can happen that the DES model already operates in the LES mode in the outer region of the boundary layer. As reported by Menter and Kuntz [46], this can lead to premature flow separation, which is therefore termed Grid-Induced Separation (GIS).

\section{Delayed Detached-Eddy Simulation - DDES}

As a remedy for the grid-induced separation, the Delayed Detached-Eddy Simulation (DDES) was developed by Spalart et al. [78]. The idea is to control the activation of the LES mode by introducing the delay function $f_{d}$, which is sensitized to the boundary layer based on the local eddy viscosity level, given by

$$
f_{d}=1-\tanh \left(\left(8 r_{d}\right)^{3}\right) \quad \text { with } \quad r_{d}=\frac{\nu+\nu_{t}}{\kappa^{2} d_{w}^{2} \max \left(\sqrt{\frac{\partial u_{i}}{\partial x_{j}} \frac{\partial u_{i}}{\partial x_{j}}} ; 10^{-10}\right)}
$$

The function $r_{d}$ is equal to one in the logarithmic region of zero-pressure-gradient boundary layers ${ }^{6}$ and goes to zero at the boundary-layer edge. With the factor of 8 in Eq. (1.47), the delay function is calibrated to be zero inside turbulent boundary layers and to smoothly approach one at the boundary-layer edge. By incorporating the function $f_{d}$ into the hybrid length scale in the DDES as

$$
l_{\mathrm{DDES}}=l_{\mathrm{RANS}}-f_{d} \max \left(0, l_{\mathrm{RANS}}-l_{\mathrm{LES}}\right),
$$

the attached boundary layer is shielded from the LES mode. The function $f_{d}$ is therefore also referred to as the shielding function. To prevent the accidental activation of the low-Reynolds terms of the SA model in LES regions, the function $\Psi$ is introduced in the definition of the LES length scale by Spalart et al. [78], which is given by

$$
l_{\mathrm{LES}}=C_{\mathrm{DES}} \Psi \Delta \quad \text { with } \quad \Psi=\min \left(100, \frac{1-\frac{c_{b 1}}{c_{w 1} \kappa^{2} f_{w}^{*}}\left(1-f_{t 2}\right) f_{v 2}}{f_{v 1} \max \left(10^{-10}, 1-f_{t 2}\right)}\right)
$$

This is not necessary for the SST-based hybrid RANS/LES approaches, as the model contains no low-Reynolds modifications.

\footnotetext{
${ }^{6}$ According to the mixing length hypothesis, the kinematic eddy viscosity in the logarithmic part of the boundary layer $\left(d_{w} / \delta<0.15\right.$ with boundary-layer thickness $\left.\delta\right)$ is given by $\nu_{t}=\kappa^{2} d_{w}^{2} \frac{\partial u}{\partial y}$
} 


\section{Improved Delayed Detached-Eddy Simulation - IDDES}

While the DES model was originally devised for massively separated flows, its field of application was quickly extended to flows with incipient separation. Moreover, test cases with growing complexity were considered. For example the behavior of hybrid RANS/LES approaches for multi-element airfoils is one major topic of the present thesis.

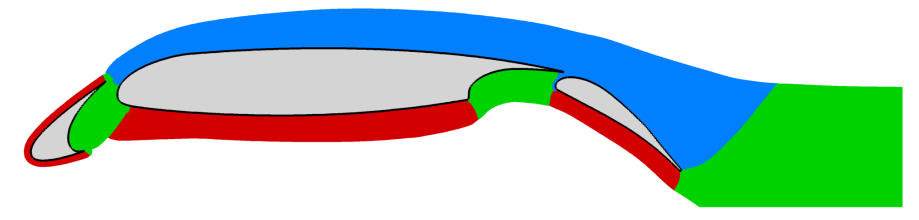

Fig. 1.3: Modeling regions around the DLR F15 three-element airfoil (red: attached flow, green: massively separated flow, blue: boundary layers with turbulent inflow).

Fig. 1.3, depicts the area around a three-element airfoil, which encloses several regions with different modeling requirements: in the green regions, the curbs of the slat and the main wing as well as downstream of the airfoil, the flow is massively separated and DES is a suited approach. In the red regions on the pressure sides of the main wing and flap, as well as at the slat, the flow is essentially fully attached; therefore, the RANS mode is appropriate. In the blue regions the boundary layers at the suction sides of the flap and the main wing are influenced by the flow upstream: as the shear layers in the wake of the slat and the main wing are treated in LES mode, resolved turbulent structures are convected into the attached boundary layers at the wing and the flap, respectively. In the present thesis wall-modelled LES is considered for the flow situation in the blue regions in Fig. 1.3.

Travin et al. [85] and Shur et al. [71] developed the Improved Delayed Detached-Eddy Simulation (IDDES) as a hybrid RANS/LES model that provides a wall-modeled LES mode. In comparison to the resolution requirements for a wall-resolved LES in Eq. (1.31), the number of grid points for a wall-modeled LES of a turbulent boundary layer is considerably reduced. Choi and Moin [10] estimate the required number of points at

$$
N_{\mathrm{wM}-\mathrm{LES}} \approx R e .
$$

The IDDES addresses a problem that was observed by Nikitin et al. [56], who enforce a wall-modeled LES behavior of the original DES model through their grid design. For the flow in a channel, the grid spacing is chosen so small that the model switches to LES in the logarithmic region of the boundary layer. The velocity in the inner region of the boundary layer is modeled by RANS, while in the outer region it is resolved by LES. At the interface a mismatch between the RANS and the LES solution is observed, which is termed the Log-Layer Mismatch (LLM). It occurs because the development of the resolved structures sets in only at a certain distance from the RANS/LES interface, while the production of modeled turbulence is stopped immediately at the interface.

In the wall-modeled LES mode of the IDDES, the activation of RANS and LES is controlled by the function $f_{B}$, which ensures the RANS mode in the inner region of the boundary layer close to the wall. It is given by

$$
f_{B}=\min \left(2 e^{-9 \alpha^{2}} ; 1\right),
$$


with $\alpha=0.25-\frac{d_{w}}{h_{\max }}$. When the wall distance $d_{w}$ becomes larger than the maximum grid-cell size $h_{\max }$, the function $f_{B}$ goes to zero. Given a sufficient grid resolution, this is usually the case within the logarithmic region of the boundary layer.

To prevent the log-layer mismatch the function $f_{e}$ is introduced, which takes values of zero almost everywhere, except, close to the RANS/LES interface

$$
f_{e}=\max \left(\left(f_{e 1}-1\right) ; 0\right) \Psi f_{e 2} .
$$

Through $f_{e}$ the level of the modeled eddy viscosity is elevated to compensate the lack of resolved turbulent structures in the LES region near the interface. Furthermore, in the IDDES a modified LES filter width is designed for the wall-modeled LES mode. It is given in Eq. (3.19) in Sect. 3.3.3.

If the resolution of the boundary layer is not sufficient for a wall-modeled LES, the IDDES falls back to the DDES model behavior and shields the whole boundary layer with a delay function. Similar to $f_{d}$ in Eq. (1.47), the delay function is defined as

$$
f_{d t}=1-\tanh \left(\left(8 r_{d t}\right)^{3}\right), \quad \text { with } \quad r_{d t}=\frac{\nu_{t}}{\kappa^{2} d_{w}^{2} \max \left(\sqrt{\frac{\partial u_{i}}{\partial x_{j}} \frac{\partial u_{i}}{\partial x_{j}}} ; 10^{-10}\right)} .
$$

If $r_{d t}$ was used in the formulation of the DDES switching function Eq. (1.47), $f_{d}$ would become 1 in a small region close to the wall, which would activate the LES mode. However, in the IDDES this gap is closed through the function $f_{B}$, which activates the RANS mode near the wall. The functions $f_{B}$ for the wall-modeled LES mode and $f_{d t}$ for the DDES mode are combined in the function $\tilde{f}_{d}$, which is given by

$$
\tilde{f}_{d}=\max \left(\left(1-f_{d t}\right), f_{B}\right) .
$$

With $\tilde{f}_{d}$, the final hybrid length scale in the IDDES is defined as

$$
l_{\mathrm{IDDES}}=\tilde{f}_{d}\left(1+f_{e}\right) l_{\mathrm{RANS}}+\left(1-\tilde{f}_{d}\right) l_{\mathrm{LES}} .
$$





\section{Chapter 2}

\section{Numerical method: DLR-TAU}

All simulations in the present thesis apply the DLR-TAU code, which mainly consists of an unstructured finite-volume flow solver, in the following referred to as TAU. For the spatial discretization the dual-grid approach is used, which is illustrated in Sect. 2.1. TAU's discretization methods for the Navier-Stokes equations are described in Sect. 2.2. Important optimizations of the spatial discretization for scale-resolving simulations are introduced in Sect. 2.3. The convergence of the iteration scheme and the statistical convergence of an unsteady simulation are discussed in Sect. 2.4. In order to realize moving grid topologies, the DLR-TAU code provides two approaches: the Chimera method, which operates on overset grids, and a grid-deformation tool. Both methods are described in Sect. 2.5.

\subsection{Discretization of the computational domain}

The computational domain $\Omega$ is discretized by a primary grid $\mathcal{X}$, consisting of hexahedral, pyramidal, tetrahedral, and prismatic elements, with structured layers near solid walls and unstructured elements in the farfield. The grid points ${ }^{1}$ are denoted as $\boldsymbol{x}_{I} \in \mathcal{X}$.

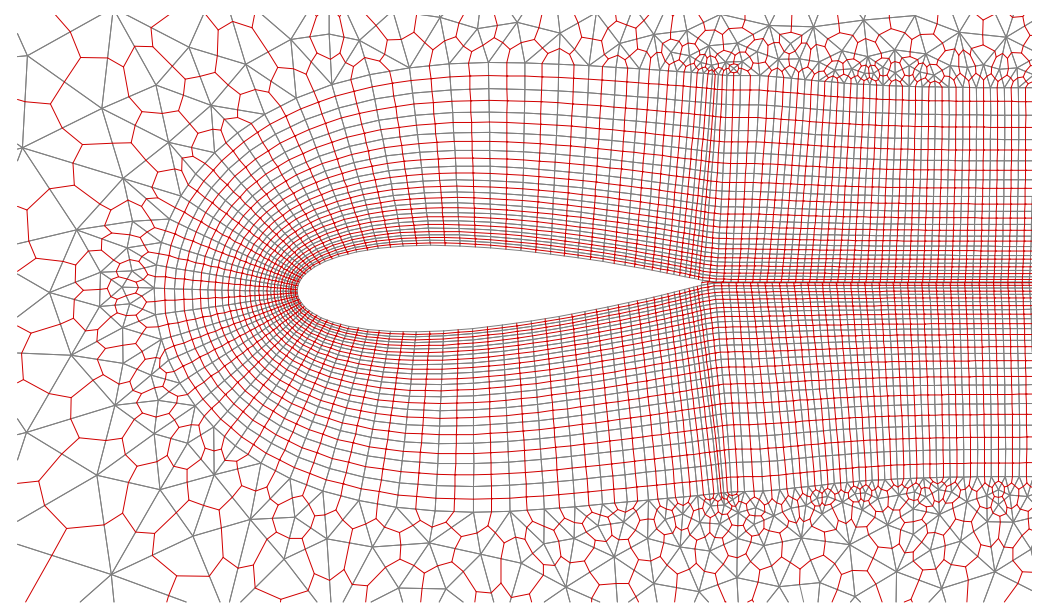

Fig. 2.1: Hybrid primary grid (black) and dual grid (red) around a NACA-0021 airfoil.

The dual grid $\widetilde{\mathcal{X}}$ is generated in a preprocessing step, where control volumes $V_{I} \in \widetilde{\mathcal{X}}$, called dual cells ${ }^{2}$, are constructed around the points $\boldsymbol{x}_{I}$. In Fig. 2.1 the dual-grid approach for the two-dimensional NACA-0021 airfoil is illustrated. The primary

\footnotetext{
${ }^{1}$ In the description of the spatial discretization capital letter are used as indices, in order to distinguish them from the indices that address the components of the vectors and tensors.

${ }^{2}$ The dual cells are constructed from facets that connect the barycenter of the edges, surfaces and volumes of the primary grid elements. The facets in the elements surrounding $\boldsymbol{x}_{I}$ build the surface of the dual cell.
} 
grid, consisting of quadrilateral and triangular primary elements, is drawn in black; the dual-grid cells are drawn in red. There is a direct correspondence between the primary grid points and the dual-grid control volumes. The union of all control volumes covers the closure of the domain

$$
\bar{\Omega}=\bigcup_{V_{I} \in \tilde{\mathcal{X}}} \overline{V_{I}} .
$$

The grid is stored in an unstructured data format, such that for each point only the neighbor points are directly accessible. The dual-grid approach has the advantage, that on the one hand the mixed element types in the primary grid offer a high flexibility during the grid generation, while on the other hand, the primary edges corresponding to dual-cell faces provide an efficient face-based data structure.

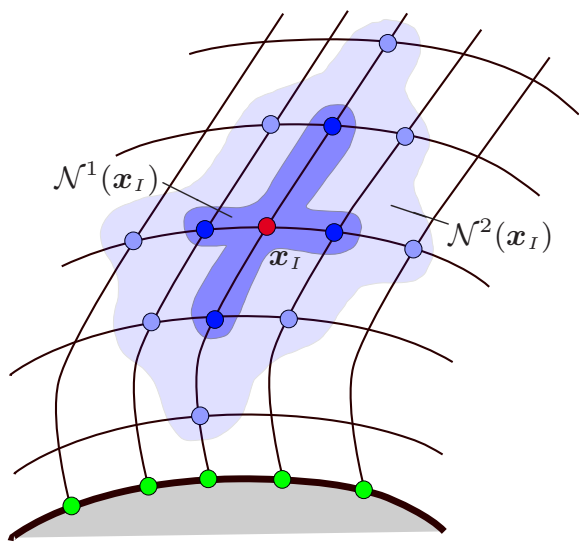

Fig. 2.2: Grid above a viscous wall.

Fig. 2.2 illustrates some terms, which are used in the following. The two-dimensional primary grid consists of quadrilateral elements, and the shaded region represents a viscous wall. The set of all grid points can be decomposed into the disjoint sets of wall points $\mathcal{X}_{\text {wall }}$ (green) and inner points $\mathcal{X}_{\text {inner }}=\mathcal{X} \backslash \mathcal{X}_{\text {wall }}$.

A unique nearest wall point is associated with every point $\boldsymbol{x}_{I} \in \mathcal{X}_{\text {inner }}$, which is the wall point with the minimal distance, denoted as

$$
f_{\mathrm{nwp}}\left(\boldsymbol{x}_{I}\right)=\min _{\boldsymbol{x}_{J} \in \mathcal{X}_{\mathrm{wall}}}\left(\left\|\boldsymbol{x}_{I}-\boldsymbol{x}_{J}\right\|\right) \text {. }
$$

The direct neighbors of the point $\boldsymbol{x}_{I}$ (red) are the points that are connected by a primary-grid edge (blue). The set of direct neighbors is denoted as $\mathcal{N}\left(\boldsymbol{x}_{I}\right)$ and is depicted by the inner shaded region. The second neighbors are the next neighbors of the direct neighbor points (light blue) in the outer shaded region, and the set of points is denoted as

$$
\mathcal{N}^{2}\left(\boldsymbol{x}_{I}\right)=\bigcup_{\boldsymbol{x}_{J} \in \mathcal{N}\left(\boldsymbol{x}_{I}\right)} \mathcal{N}\left(\boldsymbol{x}_{J}\right)
$$

Accordingly the set of third and fourth neighbors is defined.

In a local operation the evaluation of an expression is limited to the direct neighborhood. Additional communication effort is necessary to exchange information between points that are not connected by an edge, which means that a non-local operation is computationally more expensive.

\subsection{Discretization of the governing equations}

The finite-volume discretization is based on the conservative form of the NavierStokes equations. In TAU the method of lines is followed, which decouples the discretization of space and time. 


\section{Temporal discretization}

For the temporal discretization a dual-time-stepping scheme is used to solve the semi-discrete time dependent system of equations

$$
\frac{\partial \boldsymbol{w}}{\partial t}=-\mathcal{R}(\boldsymbol{w})
$$

with the vector of the conservative variables $\boldsymbol{w}=(\rho, \rho \boldsymbol{u}, \rho E)^{T}$ and the terms in the residual $\mathcal{R}(\boldsymbol{w})$ appropriately discretized in space. The implicit second-order Backward-Differencing Formula $(\mathrm{BDF}(2))$ is given by

$$
\frac{3}{2 \Delta t} \boldsymbol{w}^{n+1}-\frac{2}{\Delta t} \boldsymbol{w}^{n}+\frac{1}{2 \Delta t} \boldsymbol{w}^{n-1}=-\mathcal{R}\left(\boldsymbol{w}^{n+1}\right) .
$$

The steady-state solution $\boldsymbol{w}^{*}$ in pseudo time $t^{*}$ of the dual-time-stepping scheme

$$
\frac{\partial \boldsymbol{w}^{*}}{\partial t^{*}}+\frac{3}{2 \Delta t} \boldsymbol{w}^{*}-\frac{2}{\Delta t} \boldsymbol{w}^{n}+\frac{1}{2 \Delta t} \boldsymbol{w}^{n-1}=-\mathcal{R}\left(\boldsymbol{w}^{*}\right)
$$

also satisfies Eq. (2.5). To solve the equations in the pseudo time, the Lower-Upper Symmetric Gauss-Seidel (LU-SGS) iterative solver by Dwight [16] is used. An iteration on the LU-SGS solver is referred to as an inner iteration.

The convergence-acceleration methods for steady-state solutions can also be used with the dual-time-stepping scheme. Available acceleration techniques in TAU are locally varying time steps, residual smoothing, multi-grid methods using the full approximation scheme described by Brandt [5], and low-Mach number preconditioning. In the preconditioning the flux term is multiplied with the matrix $\boldsymbol{P}$ in order to reduce the disparity of the eigenvalues of the system, which allows the choice of larger time steps. According to Radespiel et al. [65], the preconditioning matrix for the primitive variables is given by

$$
\boldsymbol{P}=\left(\begin{array}{ccccc}
m^{2} g & 0 & 0 & 0 & -m^{2} \frac{\gamma p}{T} \delta \\
-\frac{\alpha u_{1} g}{\rho a^{2}} & 1 & 0 & 0 & \frac{\alpha u_{1}}{\rho a^{2}} \frac{\gamma p}{T} \delta \\
-\frac{\alpha u_{2} g}{\rho a^{2}} & 0 & 1 & 0 & \frac{\alpha u_{2}}{\rho a^{2}} \frac{\gamma p}{T} \delta \\
-\frac{\alpha u_{3} g}{\rho a^{2}} & 0 & 0 & 1 & \frac{\alpha u_{3}}{\rho a^{2}} \frac{\gamma p}{T} \delta \\
\frac{1}{\rho C_{p}}\left(m^{2} g-1\right) & 0 & 0 & 0 & 1-(\gamma-1) m^{2} \delta
\end{array}\right),
$$

with the free parameters $\alpha, \beta$, and $\delta$ and the derived variables $g=1+(\gamma-1) \delta$ and $m^{2}=\frac{\beta}{a^{2}}$, where $a$ is the speed of sound. The free parameter $\beta$ is chosen proportional to the local flow velocity; therefore, it approaches zero in stagnation regions. To prevent singularities of $\boldsymbol{P}$, a lower bound for $\beta$ is defined by using a cut-off factor $k_{\beta}$.

\section{Spatial discretization}

With the finite-volume discretization, the solution is piecewise constant within a control volume $V_{I}$ and represents the volume average as given in Eq. (1.39). The inviscid terms in the momentum Eq. (1.2), consisting of the convective and the pressure term, are the only relevant terms for the energy-preserving property of the discretization scheme. Only those are considered in the following. The gradient can be approximated by the Green-Gauss formula ${ }^{3}$

$$
\nabla(\rho \boldsymbol{u} \otimes \boldsymbol{u}+p \boldsymbol{I})=\frac{1}{|V|} \oint_{\delta V}(\rho \boldsymbol{u} \otimes \boldsymbol{u}+p \boldsymbol{I}) \boldsymbol{n} \mathrm{d} S,
$$

\footnotetext{
${ }^{3}$ The equations are given in vector notation to avoid double indexing. The outer product $a \otimes b$ is component-wise defined as $(a \otimes b)_{i j}=a_{i} b_{j}$
} 
with the unit matrix $\boldsymbol{I}$ and the face-normal vector $\boldsymbol{n}$. Within the control volume $V_{I}$ the discrete form of Eq. (2.8) is given by

$$
(\nabla(\rho \boldsymbol{u} \otimes \boldsymbol{u}+p \boldsymbol{I}))_{I}=\frac{1}{\left|V_{I}\right|} \sum_{\boldsymbol{x}_{J} \in \mathcal{N}\left(\boldsymbol{x}_{I}\right)}(\rho \boldsymbol{u} \otimes \boldsymbol{u}+p \boldsymbol{I})_{I J} \boldsymbol{n}_{I J}
$$

where $\boldsymbol{n}_{I J}$ is the area-weighted face-normal vector between the control volumes $V_{I}$ and $V_{J}$ as shown in Fig. 2.3. The flux tensor $(\rho \boldsymbol{u} \otimes \boldsymbol{u}+p \boldsymbol{I})_{I J}$ has to be reconstructed from the left and right states at the face.

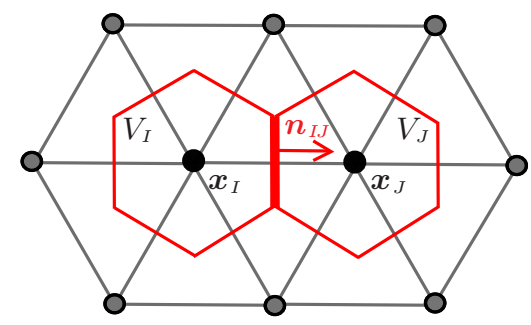

Fig. 2.3: Dual-cell face between the points $\boldsymbol{x}_{I}$ and $\boldsymbol{x}_{J}$.

For the approximation of the fluxes at the cell faces, the skew-symmetric form by $K o k[36]$ is used, which is constructed from the divergence form $D$ and the advection form $A$, given by

$$
D=\nabla(\rho \boldsymbol{u} \otimes \boldsymbol{u})+\nabla p \quad \text { and } \quad A=\rho \boldsymbol{u}(\nabla \otimes \boldsymbol{u})+\nabla p,
$$

which results in

$$
K=\frac{1}{2}(D+A)=\frac{1}{2} \nabla(\rho \boldsymbol{u} \otimes \boldsymbol{u})+\frac{1}{2} \rho \boldsymbol{u} \nabla \otimes \boldsymbol{u}+\nabla p .
$$

With this formulation the kinetic energy and the pressure are conserved both locally and globally in the computational domain. Therefore, the discretization of the convective term does not contribute to the physical dissipation, and spurious kinetic energy is neither produced nor dissipated. This is a significant improvement compared to the standard divergence form, especially in scale-resolving simulations.

Additionally, Kok [36] defines specific averaging rules for the reconstruction of the face values in the gradient operator, denoted by the symbol - . Only with this formulation the energy preserving property of the scheme can be ensured. With the skew-symmetric formulation the flux tensor at the face is approximated as

$$
(\overline{\rho \boldsymbol{u}} \otimes \overline{\boldsymbol{u}}+\bar{p} \boldsymbol{I})_{I J}=\frac{1}{2}\left(\boldsymbol{u}_{L}+\boldsymbol{u}_{R}\right) \otimes \frac{1}{2}\left(\rho_{L} \cdot \boldsymbol{u}_{L}+\rho_{R} \cdot \boldsymbol{u}_{R}\right)+\frac{1}{2}\left(p_{L}+p_{R}\right) \boldsymbol{I},
$$

where the values of $\boldsymbol{u}_{L}$ and $\boldsymbol{u}_{R}$ are reconstructed from the "left" control volume $V_{I}$ and the "right" control volume $V_{J}$, respectively. In a second-order central scheme, they are given by the respective cell values, i.e. $\boldsymbol{u}_{L}=\boldsymbol{u}_{I}$ and $\boldsymbol{u}_{R}=\boldsymbol{u}_{J}$. The same holds for the pressure and the density. The skew-symmetric formulation of the mass and energy equations is described by Löwe et al. [42] and in Probst et al. [H].

To stabilize the non-dissipative central discretization of the convective fluxes, a fourth-order matrix-valued artificial dissipation is added to damp high-frequency oscillations. The dissipative flux at the face between $V_{I}$ and $V_{J}$ is given by

$$
\boldsymbol{D}_{I J}^{(4)}=k^{(4)} \cdot\|\boldsymbol{P} \boldsymbol{A}\|_{I J} \cdot \psi_{I J} \cdot\left(\nabla^{2} \boldsymbol{u}_{I}-\nabla^{2} \boldsymbol{u}_{J}\right) .
$$


The value $k^{(4)}$ is the global scaling factor of the artificial dissipation. The cellstretching coefficient $\psi_{I J}$ takes into account the aspect ratio of the grid cells, because the computational stability is enhanced if the artificial dissipation is increased on anisotropic grids. The matrix $\boldsymbol{A}$ is the artificial dissipation operator, and $\boldsymbol{P}$ is the preconditioning matrix from Eq. (2.7). Radespiel et al. [65] show, that it is necessary to include $\boldsymbol{P}$ in Eq. (2.13), to ensure, that the solution of the compressible Navier-Stokes equations converges to the solution of the incompressible equations for $M a \rightarrow 0$.

Turkel [86] states that it is possible to use two different preconditioning matrices in the time discretization and in the artificial dissipation. Therefore, in the current simulations two different cut-off values $k_{\beta, 1}$ and $k_{\beta, 2}$ are used, where $k_{\beta, 1}$ is optimized to accelerate the convergence, while $k_{\beta, 2}$ is tuned for optimal accuracy.

\subsection{Optimization of the numerical scheme for scale- resolving simulations}

Being originally developed as a RANS solver for industrially relevant applications, the primary focus of TAU has been on stability in complex flow problems. For the purpose of scale-resolving simulations, it is necessary to re-assess the accuracy of the numerical scheme.

\section{Low-dissipation properties}

In an unstructured finite-volume solver, the application of discretization schemes with higher than second order is difficult. Therefore, in Probst and Reuß [G] the influence of the artificial dissipation on scale-resolving simulation is investigated in combination with the skew-symmetric second-order discretization, according to Eq.(2.11). A termby-term calibration of the artificial dissipation in Eq. (2.13) is presented to optimize the settings for scale-resolving simulations.

The test case of a fully-developed turbulent channel flow is used, which is presented in Sect. 4.2. To minimize the interference with the turbulence model, the calibration simulations are performed as wall-resolved LES using the Smagorinsky and the WALE sub-grid models, described in Sect. 1.3.2. It is confirmed that a certain amount of artificial dissipation is necessary to stabilize the simulation. In simulations with $k^{(4)}=0$, odd-even decoupling of the solution in the span-wise direction is observed. If the value is chosen too high, the dissipation damps the resolved structures and corrupts the results. A value of $k^{(4)}=\frac{1}{1024}$ is found to be optimal with the implementation of Eq. (2.13) in TAU.

The cell-stretching coefficient $\psi_{I J}$ is supposed to increase the artificial dissipation in highly stretched cells, which are typically used very close to the wall in RANS simulations. For isotropic cells the influence of $\psi_{I J}$ vanishes. However, if in scaleresolving simulations the grid cells are anisotropic in the LES regions, the amount of artificial dissipation might be overly increased. All presented simulations ran stable with $\psi_{I J}=1$, which is the most accurate choice for scale-resolving simulations.

The optimal cut-off value for the preconditioning of the artificial dissipation is found to be $k_{\beta, 2}=0.3$. The set of optimized values for all factors in Eq. (2.13) with respect to low dissipation is termed the LD settings in the following. 


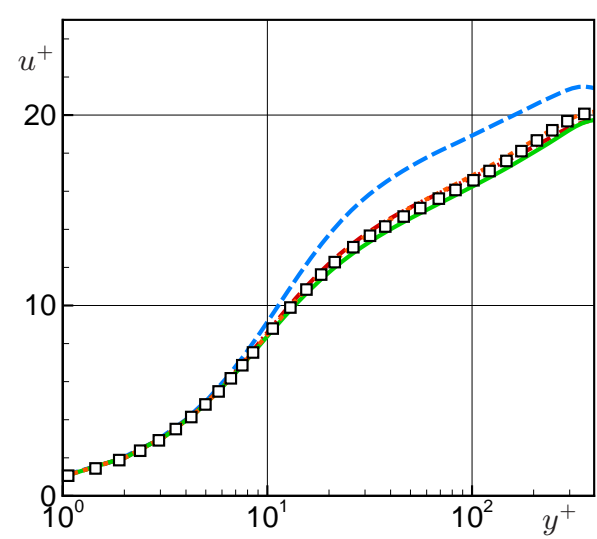

(a) Velocity $u^{+}\left(y^{+}\right)$

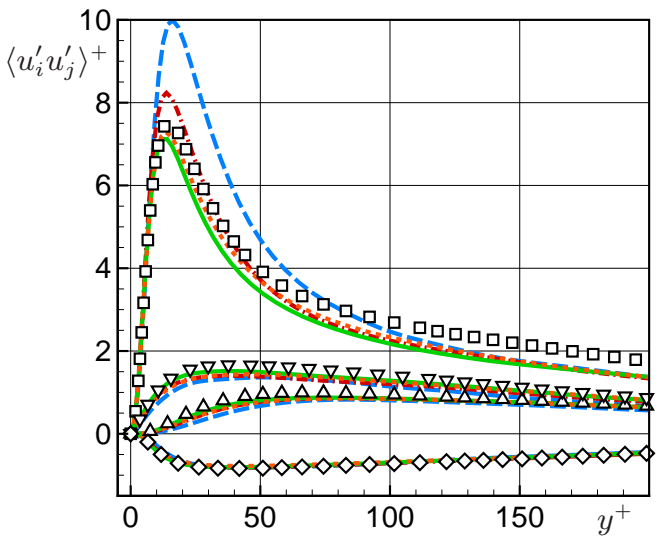

(b) Reynolds stresses $\left\langle u_{i}^{\prime} u_{j}^{\prime}\right\rangle$

Fig. 2.4: Wall-resolved LES of a turbulent channel flow at $R e_{\tau}=395$, reproduced from Probst et al. $[\mathrm{H}]:$ ( - - Reference numerics (WALE); (-.-.-) LD scheme (WALE); (—) LD2 scheme (WALE); (- - -) LD2 scheme (Smagorinsky) $(\square)$ DNS data.

In Fig. 2.4 the velocity profile and the Reynolds shear stresses in the plane-channel flow at $R e_{\tau}=395$, which are obtained in wall-resolved LES simulations, are shown. The LD settings lead to a much better agreement with DNS results by Moser et al. [52] than the standard TAU settings. The effect of the LD settings on the wallmodeled LES capabilities of TAU is assessed in Probst and Reuß [G], and some results are presented in Sect. 4.2.

\section{Low-dispersion properties}

Besides numerical dissipation the discretization scheme is also afflicted with a dispersion error. While the dissipation error is an error in the amplitude, the dispersion error leads to an error in the wave length. Löwe et al. [42] present a new formulation for the central discretization of the convection term that aims at minimizing the dispersion error. To achieve this, the discretization stencil is increased by including the gradients in the neighboring cells. In Eq. (2.12) the states $\boldsymbol{u}_{L}$ and $\boldsymbol{u}_{R}$ are reconstructed as

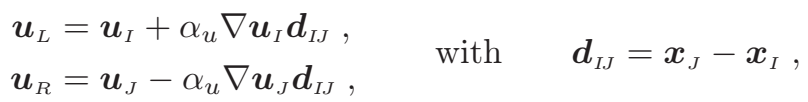

where $\nabla \boldsymbol{u}_{I}$ and $\nabla \boldsymbol{u}_{J}$ are given by the Green-Gauss gradients in the control volumes $V_{I}$ and $V_{J}$, respectively. With $\alpha_{u}=0$ the standard second-order central scheme is obtained, whereas $\alpha_{u}=1 / 3$ gives a fourth-order scheme on smooth isotropic grids. The idea of the low-dispersion formulation is to use the additional degree of freedom to optimize the dispersion property, rather than increasing the order of the scheme. A numerical investigation by Löwe et al. [42] shows that a value of $\alpha_{u}=0.36$ leads to a scheme that is optimized with respect to the dispersion. They also answer the question for which variables Eq. (2.14) should be applied.

Together with the LD settings, which were presented above, this combined lowdissipation low-dispersion scheme is termed the LD2 scheme. Löwe et al. [42] compare 
the LD2 scheme with the standard second-order scheme for the convection of an isentropic vortex on a highly deformed grid and for the Taylor-Green Vortex. In both cases the LD2 scheme is superior to the standard scheme in preserving the vortex dynamics. In Fig. 2.4 also results for the LD2 scheme are shown. For the higher-order statistics, represented by the Reynolds stresses, the LD2 scheme leads to improved results for both, the Smagorinsky and the WALE model, in comparison to the low-dissipation settings alone.

Probst et al. $[\mathrm{H}]$ apply the LD2 scheme to further test cases, and in most cases the results are improved significantly. They also propose a hybrid blending of the standard settings for RANS and the LD2 scheme for LES, in order to prevent instabilities in the RANS regions that occur using the LD2 scheme in hybrid RANS/LES simulations of the DLR F15 three-element airfoil. Since an optimal blending scheme has still been under investigation, the LD2 scheme is not used during the preparation of the target application in Chap. 4. Instead, most of the presented simulations apply the low-dissipation settings.

\subsection{Convergence criteria and averaging}

In the dual-time-stepping scheme, Eq. (2.6), for each physical time step a pseudo steady state with $\mathcal{R}(\boldsymbol{w})=\mathbf{0}$ has to be obtained within the inner iterations. In practice the iterations are stopped if $\|\mathcal{R}(\boldsymbol{w})\|<\epsilon$, where the smallest reachable $\epsilon$ is determined by the machine accuracy; however, in realistic complex geometries machine accuracy is hardly ever reached. In Reuß et al. [C] (see Appx.) the number of inner iterations was fixed, and it had to be chosen very high, to ensure a reduction of the residual by at least three orders of magnitude in all physical time steps. Since in most of the steps a smaller number would have been sufficient, it is desirable to provide suitable convergence criteria for unsteady flows that terminate the inner iterations automatically.

In TAU the Cauchy criterion is available to measure the convergence of a variable $\phi$. With $\phi^{(n)}$ denoting the value in the $n$-th inner iteration and the user-defined tolerance threshold $\epsilon_{\text {conv }}$, convergence is reached if in $N$ consecutive inner iterations the relative changes in $\phi$ fulfill

$$
\frac{\left|\phi^{(n)}-\phi^{(n-k)}\right|}{\left|\phi^{(n)}\right|} \leq \epsilon_{\mathrm{conv}}, \quad \forall k=1, \ldots,(N-1) .
$$

Several variables can be chosen for the convergence evaluation, and it is possible to activate more than one, so that each variable must fulfill the Cauchy criterion before the iterations are stopped.

In RANS simulations the lift coefficient $C_{l}$ and the drag coefficients $C_{d}$ are typically chosen to evaluate the convergence. In the hybrid RANS/LES simulations in the present thesis, the attached flow close to the wall is mainly treated in the RANS mode and is rather stable in these regions, whereas the LES regions farther away from the wall have smaller influence on the surface forces, which are represented by $C_{l}$ and $C_{d}$. For these cases the Cauchy criteria were extended to variables that reflect the modeled or the resolved turbulence, so that they are better suited to assess the convergence in LES regions. The values $\phi$ are obtained either as the volume-weighted 
average or as the maximum over all grid points

$$
\phi_{\mathrm{avg}}=\frac{1}{\sum_{\boldsymbol{x}_{I} \in \mathcal{X}}\left|V_{I}\right|} \sum_{\boldsymbol{x}_{I} \in \mathcal{X}}\left|V_{I}\right| \phi_{I} \quad \text { or } \quad \phi_{\max }=\max _{\boldsymbol{x}_{I} \in \mathcal{X}} \phi_{I} .
$$

In the applications in Chap. 4 the mean resolved turbulent kinetic energy $k_{\text {avg }}^{\prime}$, the mean magnitude of the vorticity $\|\boldsymbol{\omega}\|_{\text {avg }}$, and the maximum eddy viscosity $\mu_{t, \max }$ are used simultaneously.

While the Cauchy criterion reflects the convergence of the pseudo-steady inner iterations, in scale-resolving simulations the statistical convergence in the physical time is of importance, too. The physical time is specified in convective time units $t_{\text {ctu }}=\frac{l}{u_{\infty}}$, where $u_{\infty}$ is the free-stream velocity and $l$ is a characteristic dimension of the geometry. For an airfoil $t_{\text {ctu }}$ denotes the overflow time, for example. Starting from the initial flow field, it takes several convective time units until the resolved structures are fully developed. This phase of the simulation is called the initial transient phase. Only after the transient phase is passed, meaningful averages can be obtained for the statistical evaluation of the simulation and for the comparison with experimental references, which often consist of temporally averaged data only.

In macroscopically stationary processes, the mean value in the $n$-th physical time step is computed as:

$$
\langle\phi\rangle_{t_{n}}=\langle\phi\rangle_{t_{n-1}}+w \cdot\left(\phi\left(t_{n}\right)-\langle u\rangle_{t_{n-1}}\right), \quad \text { with } \quad w=\frac{1}{n} .
$$

For relevant mean values, the number of samples has to be sufficient. It was found that at least five convective time units are necessary for the first and second order moments to converge within a given tolerance. In Reuß et al. [B] (see Appx.) up to $40 t_{\text {ctu }}$ were computed until statistical convergence was reached. In order to obtain mean values for macroscopically non-stationary processes in experiments, ensemble averages or, for periodic flows, phase averages are usually used. Because of the large computational effort of even one single simulation, these approaches are unfeasible for hybrid RANS/LES simulations.

To obtain an approximation of the mean values that is able to represent the macroscopic dynamics of the system, a weighted moving-average formulation is used. To this end, the weighting factor $w$ in Eq. (2.17) is replaced by $w=\frac{2}{s+1}$, with a constant user-defined value $s$, which lets the influence of the old mean values decay exponentially. This procedure is used in Reuß et al. [E] (see Appx.) to obtain mean values for an airfoil-generated vortex, which is convected through a wind tunnel. In order to improve the second-order statistical moments, the homogeneity of the flow in the span-wise direction is exploited: the mean values in Eq. (2.17) are additionally averaged in the span-wise direction before the variances are computed. As a consequence, the effective number of samples is increased.

\subsection{Chimera method and deformation tool}

In practical aeronautical applications the whole geometry or parts of it can be in motion relative to a fixed reference system. To realize this situation in a flow simulation, TAU provides two different approaches: with the Chimera technique, implemented in TAU by Madrane et al. [44], the geometry is discretized using overlapping grids, which can be in translatory or rotational motion relative to each other. With the 
deformation technique, implemented in TAU by Heinrich et al. [25], radial basis functions are used to modify the grid. In the present thesis both approaches were tested to identify their strengths and weaknesses in the considered applications. To illustrate the differences between the two approaches, Fig. 2.5 shows how both methods are utilized to realize the rotation of a NACA-0021 airfoil in a wind tunnel.

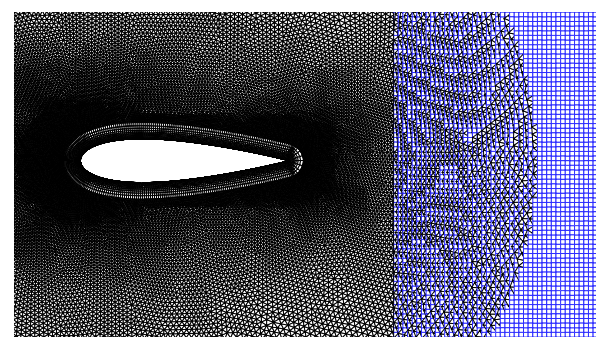

(a) Chimera at $\alpha=0^{\circ}$

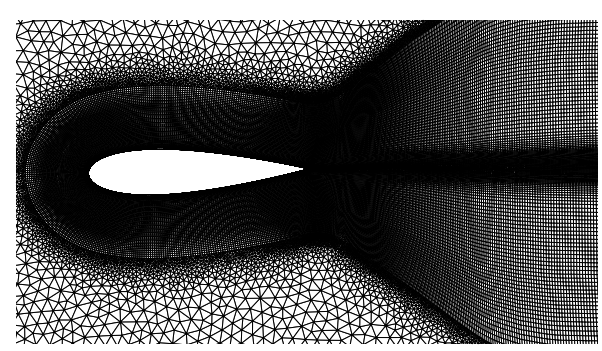

(c) Deformation at $\alpha=0^{\circ}$

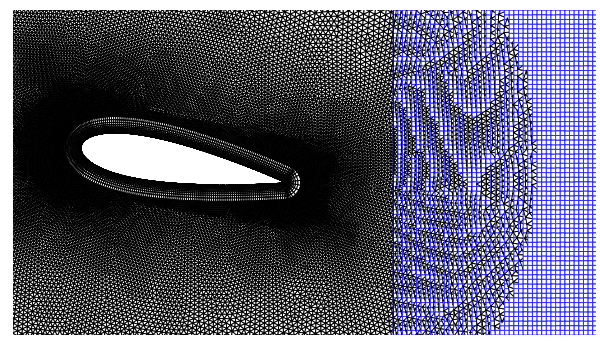

(b) Chimera at at $\alpha=10^{\circ}$.

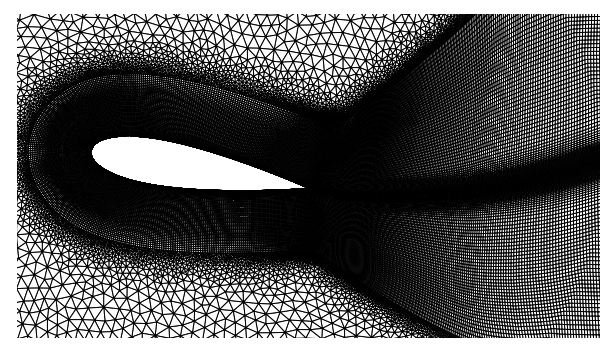

(d) Deformation at at $\alpha=10^{\circ}$.

Fig. 2.5: Comparison of the Chimera approach and grid deformation for the rotation of a NACA-0021 airfoil.

With the Chimera approach, utilized in Reuß et al. [A] (see Appx.), the computational domain $\Omega$ is decomposed into overlapping sub-domains $\Omega_{i}$. The equations are solved independently on each grid block, such that $\boldsymbol{w}_{i}$ is the solution of

$$
\frac{\partial}{\partial t} \boldsymbol{w}_{i}+\mathcal{R}\left(\boldsymbol{w}_{i}\right)=0 \quad \text { in } \Omega_{i}
$$

The solutions $\boldsymbol{w}_{i}$ and $\boldsymbol{w}_{j}$ in two overlapping blocks $\Omega_{i}$ and $\Omega_{j}$ are coupled through boundary conditions at the borders of the overlap region $\Omega_{i} \cap \Omega_{j}$, which are denoted as $\Omega_{1 \leftarrow 2, i p}$ and $\Omega_{2 \leftarrow 1, i p}$. For each point $\boldsymbol{x}_{1}$ in the interpolation region $\Omega_{1 \leftarrow 2, i p}$, belonging to the grid $\mathcal{X}_{1}$, an appropriate donor element in the primary grid $\mathcal{X}_{2}$ is determined with an Alternating-Digital-Tree (ADT) search algorithm. From the corner points of the respective element, the value in $\boldsymbol{x}_{1}$ is obtained from a linear, bilinear or trilinear interpolation, depending on the element type. In Figs. 2.5(a) and (b) two grid blocks are shown in black and blue, respectively.

If the blocks are in motion relative to each other, the points where the coupling conditions are applied have to be recomputed after each time step. On the other hand the grid metric is preserved during the motion and the control volumes and point distances remain unchanged. Disadvantages of the Chimera approach are the increased computational costs for the interpolation algorithm and the additional interpolation error. In order to keep the error as small as possible, the grid cells in both blocks in the overlap region should be of similar size.

In Reuß et al. [A] (see Appx.) the Chimera approach is successfully applied to simulate the interaction of an airfoil-generated vortex with a high-lift airfoil. However, 
Wawrzinek et al. [87] observed in similar simulations that the interpolation error had a similarly large effect as the disturbance under investigation. Therefore, in all further simulations that were performed during the present thesis the deformation tool was used to realize the grid motion.

With the deformation tool, which is applied in Reuß et al. [F] (see Appx.), the grid points in the original grid are moved, such that the deformed grid reflects the new position of the geometry. To this end, the deflection of a set of $N$ scattered data points with the coordinate triples $\left(x_{i}, y_{i}, z_{i}\right)$ is computed. Then the deflection is interpolated to the grid points with the coordinates $(x, y, z)$ using the radial basis function

$\Delta x(x, y, z)=\alpha_{1}+\alpha_{2} x+\alpha_{3} y+\alpha_{4} z+\sum_{i=1}^{N} \beta_{i} \sqrt{\left(x-x_{i}\right)^{2}+\left(x-x_{i}\right)^{2}+\left(x-x_{i}\right)^{2}}$,

and the interpolation coefficients $\alpha_{1}, \alpha_{2}, \alpha_{3}, \alpha_{4}$, and $\beta_{i}$. The procedure for the wall-normal and span-wise deflections $\Delta y$ and $\Delta z$ is similar.

One disadvantage of the deformation approach is the increased computational effort, because after each deformation step a new dual grid must be generated to update the metric information, such as dual-cell volumes and wall distances. However, since the motion of the grid points is taken into account as an additional grid-induced contribution to the flow velocity, see Heinrich et al. [25], no additional effort is necessary during the solver step. 


\section{Chapter 3}

\section{A grid-adaptive algebraic hybrid RANS/LES model}

In this chapter the central achievements of the present thesis are presented, and implementation details are described. In Sect. 3.1 the status of the hybrid RANS/LES approaches in TAU at the beginning of the work is recapitulated and the motivation for further improvements of the methodology is pointed out. In Sect. 3.2 the issue of grid resolution in scale-resolving simulations is addressed, with particular focus on the local refinement of the grid. In Sect. 3.3 a detailed description of the new developments in the algebraic hybrid RANS/LES model is given.

\subsection{Motivation}

One major obstacle for the applicability of the scale-resolving approaches is the long computing time, which is determined by the available hardware. The estimations that are presented in Reuß et al. [C] (see Appx.) reach values up to the order of years for the wall-modeled LES of a full aircraft configuration, for example. It is therefore very important to make a sensible use of the available resources by adjusting the grid point distribution to the flow problem. Spalart [74] formulates guidelines for the grid design for a DES by identifying regions with different resolution requirements. While this helps with the initial grid design, a sufficient grid resolution is not guaranteed. The grid resolution must be sufficient to minimize the numerical discretization error. Beyond that, the grid-cell size also determines the turbulent length scale of the LES model, as described in Sect. 1.3.2. Therefore, the grid must be suited to resolve the relevant turbulent scales. To eliminate the discretization error, a grid convergence study can be performed by refining the grid. This is done, for example, in Reu $\beta$ et al. [A] (see Appx.) for RANS simulations of the transport of a generic vortex. But the high computational costs of the scale-resolving simulations require means to assess the grid resolution using a single-computation approach. The present thesis aims at providing a procedure that does not only assess the grid, but also performs a local grid refinement if necessary. To this end, a resolution indicator is proposed, which can be used as input for the DLR-TAU grid-adaptation tool.

Furthermore, uncertainties arise from the hybrid RANS/LES models themselves. Probst et al. [63] report severe deficiencies of DDES and IDDES, both based on the SA model: for the test case of the HGR01 airfoil with incipient trailing-edge separation the authors observe massive grid-induced separation for both hybrid RANS/LES models. They conclude that the shielding functions $f_{d}$ from Eq. (1.47) and $f_{d t}$ from Eq. (1.53), respectively, fail to detect the boundary-layer edge correctly in regions of large adverse pressure gradients. By increasing the constant factor from 8 to 16 in Eq. (1.47), the modified DDES shielding function $f_{d}$ works reliably again, so that grid-induced separation is prevented. But with this approach, termed DDES 16 , the separation is fully treated in RANS mode, such that essentially a RANS result is 
obtained. The results are shown in Fig. 3.1, with permission of the original authors. The experimental data are taken from Wokoeck et al. [91].

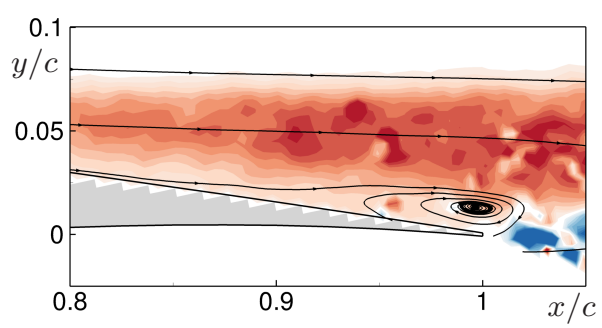

(a) PIV

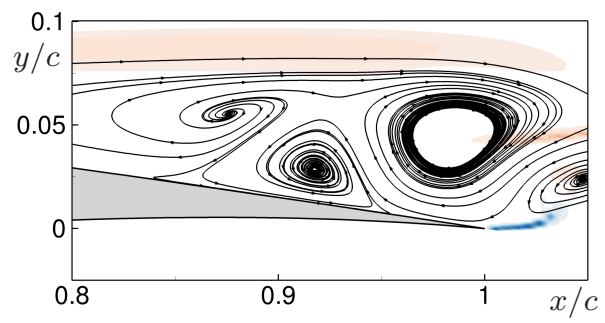

(c) SA-DDES

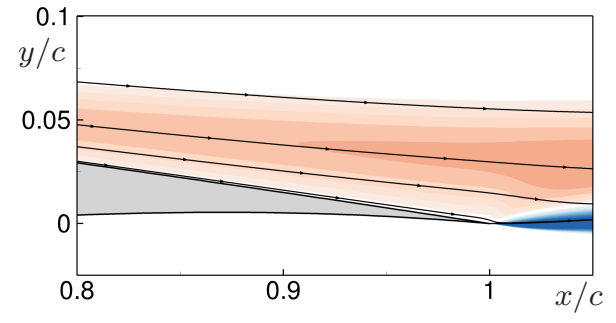

(b) SA-RANS

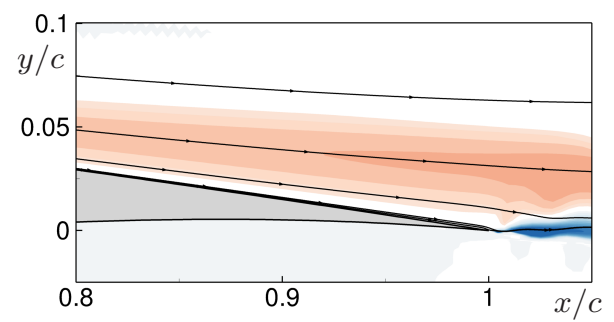

(d) SA-DDES 16

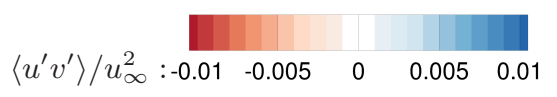

Fig. 3.1: Streamlines and Reynolds shear stress at the trailing edge of the HGR01 airfoil at $R e=0.65 \times 10^{6}, \alpha=12^{\circ}$.

The findings led to the development of the Algebraic Delayed DES (ADDES), which was for the first time applied by Knopp and Probst [34]. Including and extending the original idea of the ADDES, the following requirements provide the foundation of the present thesis:

(R1) Grid-induced separation should be prevented.

(R2) Separated regions should be treated in the LES mode.

(R3) The grey-area at the transition from RANS to LES should be reduced.

(R4) The transition from LES to RANS needs special treatment.

The turbulence model should detect the different states of the flow automatically and adapt the model behavior. As stated above, the detection must go beyond the functionality that is provided by the shielding functions in DDES and IDDES. Therefore, in the ADDES the boundary-layer velocity profiles are evaluated to provide algebraic sensors. By using the sensors to modify the shielding functions, the desired model behavior is ensured. In Fig. 3.2 the regions that are addressed by the requirements for the ADDES are exemplarily marked for the flow over a wall-mounted hump.

Concerning requirement (R1) two different flow situations can be distinguished: attached boundary layers without turbulent content in the inflow and attached boundary layers with turbulent content in the inflow. In attached boundary layers without turbulent inflow, the RANS mode should be active in the whole boundary layer, 


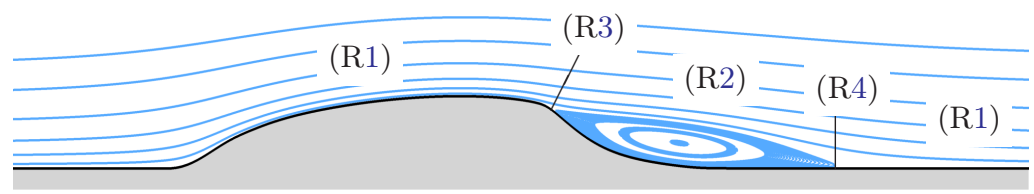

Fig. 3.2: Mean streamlines for the flow over a wall mounted hump. The regions that lead to the requirements for the ADDES are marked.

which is the purpose of the shielding functions. To support the shielding functions, in the original implementation of the ADDES a sensor is implemented that detects the boundary-layer edge and enforces the RANS mode up to the edge of the boundary layer. For attached boundary layers with turbulent content in the inflow, the wall-modeled LES mode was introduced in the IDDES. To include this mode in the ADDES, it is essential to couple the algebraic sensors with the IDDES. To distinguish the two situations, a sensor for wall-modeled LES has been added to the original ADDES formulation, see Sect 3.3.2. Another sensor of the original ADDES is designed to detect separated flow regions, in order to meet requirement (R2).

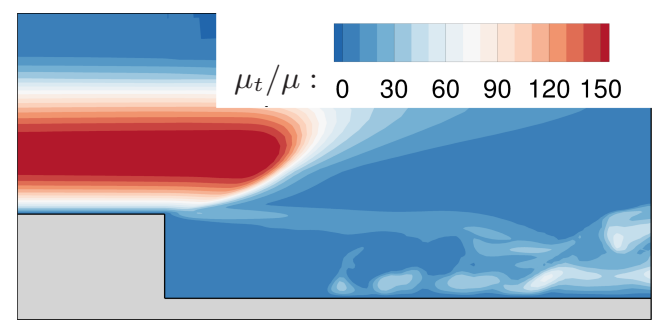

Fig. 3.3: Eddy-viscosity distribution for a SA-based DDES of the backward-facing step flow.

In requirement (R3) the grey-area problem is addressed, which is inherent in the hybrid RANS/LES approaches. In Fig. 3.3 it is illustrated with the help of the flow over a backward-facing step: the boundary layer on the step is treated in the RANS mode, and the flow is characterized by a high level of eddy viscosity $\mu_{t}$. At the step the model switches from RANS to LES, but the modeled $\mu_{t}$ is convected from the RANS region into the LES region. There it decreases and is gradually replaced by resolved turbulent structures. The transition region is termed the grey area, because the model behavior lies "between" RANS and LES. Without further means, the development of resolved structures starts with a delay, which may have an unfavorable effect on the solution as shown in Sect. 4.3.

The problem is approached from two directions. In the first approach the switching of the model is adjusted: to allow for a rapid generation of resolved LES content, an improved length scale is developed. In the second approach synthetic turbulent fluctuations are introduced in the flow in order to foster the generation of resolved LES content. The synthetic turbulence is a field of research on its own, which is, in the framework of the ADDES, investigated by François and Radespiel [17].

Requirement (R4) arises from situations, where the grid provides only localized LES resolution. In the example in Fig. 3.2, the grid resolution downstream of the reattachment point might not be sufficient for a wall-modeled LES. In this case the model should automatically switch to the RANS mode in the reattached flow. But the resolved turbulent structures from the recirculation region are continuously convected into the attached boundary layer. Therefore, in the ADDES special sponge 
sources have been tested, which transfer the turbulent kinetic energy from the resolved structures into modeled turbulent kinetic energy in the RANS region, see Sect 3.3.5.

Another important requirement is also addressed in the current implementation of the ADDES: the algorithm has to run efficiently and fully parallelized on multiple CPU cores to allow for large application cases. For example the target application in Sect. 4.7 took 50 days of computing time on 290 cores.

\subsection{Grid adaptation}

To minimize the discretization error, clustering of the grid points is necessary in regions of steep gradients and vortical structures. However, in complex flow situations the distribution of the gradients is not known in advance; therefore, the grid adaptation tool of the DLR-TAU code by Alrutz and Orlt [4] can be used to refine the grid locally. The refined regions are controlled by a solution-based indicator function, which is evaluated at each grid point.
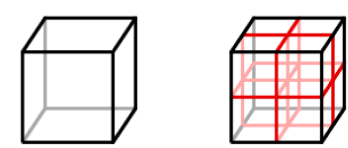

regular subdivision

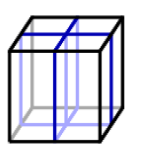

semi-regular subdivision

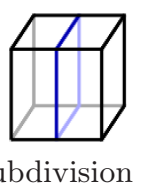

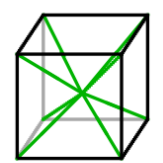

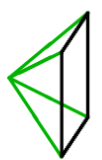

non-regular pyramidal subdivision

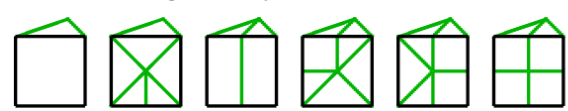

further subdivision of pyramidal parts

Fig. 3.4: Bridging elements in the refinement of the hexahedral elements.

Based on the distribution of the indicator values, the edges of the grid elements are marked for bisection. Based on this marker, the grid elements are decomposed into smaller elements, where the applicable refinement case depends on the number of bisected edges. In the example in Fig. 3.4, regular and non-regular hexahedral subdivisions are depicted. For the regular and semi-regular subdivisions the grid structure is maintained as the hexahedron is decomposed into smaller hexahedra. In the non-regular subdivision, additional unstructured pyramidal and tetrahedral elements are inserted into the grid. Corresponding subdivision cases exist for the prismatic, pyramidal, and tetrahedral elements.

\subsubsection{Span-wise grid adaption}

In the present thesis almost all geometries under study are quasi two-dimensional, which means that they are invariant under translations in the span-wise direction. For these special geometries a three-dimensional grid can be generated by stacking two-dimensional grids in the third, i.e. span-wise, direction. The number of points in the span-wise direction $N_{z}$ is determined by the resolution requirement in the focus LES region, while the total number of points in the grid grows linearly with $N_{z}$. To reduce the computational costs, the adaptation tool has been customized in order 
to restrict the span-wise refinement to user-defined regions, that should fully enclose the expected LES region including a safety margin.

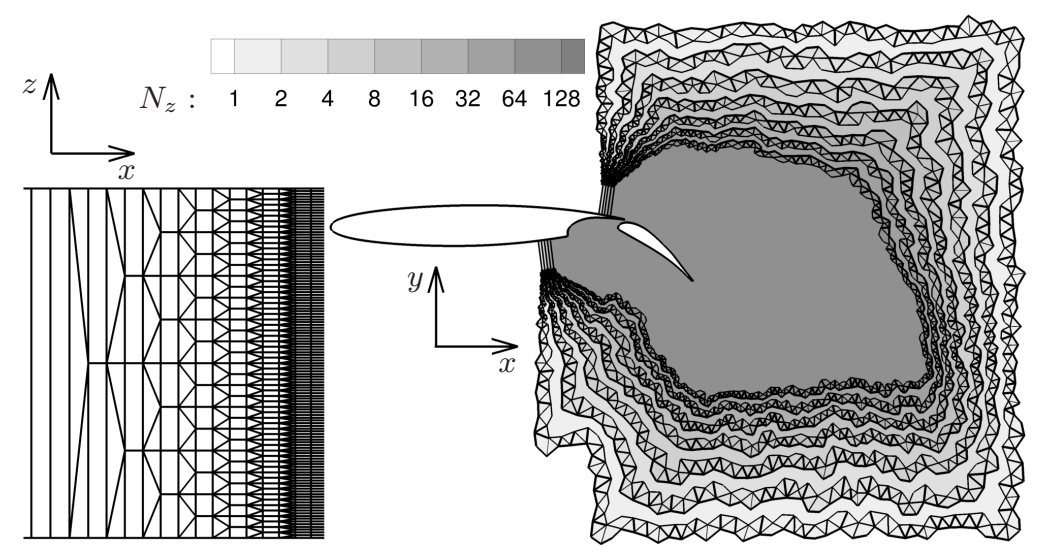

(a) Surface refinement (top view)

(b) Cut through grid

Fig. 3.5: Span-wise grid adaptation for the DLR F15 two-element airfoil to locally increase the number of grid points in the LES region.

In Fig. 3.5 the application of the span-wise adaptation for the DLR F15 two-element airfoil is shown. On the main wing the attached boundary layer is treated in RANS mode and one cell in the span-wise direction, $N_{z}=1$, is sufficient to resolve the two-dimensional solution. Close to the trailing edge of the main-wing element the resolution is increased to $N_{z}=128$ equidistant cells and transition elements are introduced. In Fig. 3.5 (b) the transition elements in the refinement region are outlined in a span-wise cut. The number of cells in the span-wise direction is indicated by the gray shades. In Fig. 3.5 (a) the transition elements on the surface of the main wing are shown. Within seven refinement steps the number of cells in the span-wise direction is increased from one to 128, and the quadrilateral elements on the surface are split into bridging triangles. To moderate the growth rate of the cell sizes, one layer of unrefined elements is inserted between two consecutive refinement steps. In the locally span-wise adapted grid $35 \%$ of points are saved compared to a grid that globally provides the high span-wise resolution without loss of numerical accuracy.

The span-wise adaptation has successfully been applied in Reuß et al. [F] (see Appx.) to refine the transport region of the lateral vortex, see also Sect. 4.6. It was also used during precursor simulations of the flow over a backward-facing step in Reu $\beta$ et al. [D] (see Appx.). However, in this case it was discarded because of difficulties at the LES-to-RANS interface, which are discussed in Sect. 3.3.5.

\subsubsection{Grid-resolution sensors}

The original TAU adaptation indicators are suited to adapt the grid to the mean flow features in steady RANS simulations. However, for scale-resolving simulations the grid must be adapted to represent the unsteady resolved scales, instead. The new points have to be introduced in regions where the LES model is active and small turbulent structures exist, which are not sufficiently resolved. The detection of the regions that are to be refined should be obtained in a single-simulation approach, in order to minimize the computational effort. 
Some preliminary approaches for the assessment of the grid resolution are presented in Reuß et al. [C] (see Appx.): the RANS length scale of a precursor RANS simulation is used to obtain an a-priori estimation of the expected turbulent length scales. The boundary-layer resolution is assessed by the ratio of the resolved Reynolds shear stress to the modeled Reynolds shear stress. The ratio of the velocity fluctuations in stream-wise and wall-normal direction is also used as an indicator of the boundarylayer resolution.

Pope [60] states that the resolved turbulent kinetic energy $k_{\text {res }}$ should amount to at least $80 \%$ of the total turbulent kinetic energy $k_{\text {tot }}$. In the context of LES the total turbulent kinetic energy $k_{\text {tot }}$ is the sum of the resolved part $k_{\text {res }}$ and the sub-grid part $k_{\text {sgs }}$, stemming from the LES sub-grid model. Thus, a grid-resolution sensor can be defined as the ratio

$$
S=\frac{k_{\mathrm{res}}}{k_{\mathrm{tot}}}=\frac{k_{\mathrm{res}}}{k_{\mathrm{res}}+k_{\mathrm{sgs}}} .
$$

With the mean velocity $\langle\boldsymbol{u}\rangle$ according to Eq. (2.17), the resolved turbulent kinetic energy can be directly computed as

$$
k_{\text {res }}=\frac{1}{2}\langle\boldsymbol{u}-\langle\boldsymbol{u}\rangle\rangle^{2} .
$$

However, for the calculation of $k_{\mathrm{sgs}}$ an appropriate model is required. In a test version in Reuß et al. $[\mathrm{C}]$ (see Appx.) the modeled $k$ is taken from a precursor RANS computation. In the present thesis several approaches to obtain $k_{\mathrm{sgs}}$ from the scaleresolving simulation are tested and compared. If the turbulence model provides a transport equation for $k$, like the $k-\omega$ SST model, one possibility is to use the averaged value $\langle k\rangle$. Alternatively, Lilly [41] gives the following relation between the eddy viscosity $\nu_{t}$ and $k_{\text {sgs }}$

$$
k_{\mathrm{sgs}}=\left\langle\nu_{t}\right\rangle^{2} /(c \Delta)^{2}, \quad \text { with } \quad c=0.094 .
$$

Knopp et al. [35] propose to use the explicitly filtered velocity $\overline{\boldsymbol{u}}$ to obtain the sub-grid velocity $\boldsymbol{u}_{\text {sgs }}=\boldsymbol{u}-\overline{\boldsymbol{u}}$. Then $k_{\text {sgs }}$ can be estimated as

$$
k_{\mathrm{sgs}}=\frac{1}{2}\left\langle\boldsymbol{u}_{\mathrm{sgs}}\right\rangle^{2} .
$$

To obtain the explicitly filtered velocity $\overline{\boldsymbol{u}}$, the solution is smoothed within the direct neighborhood $\mathcal{N}^{1}\left(\boldsymbol{x}_{I}\right)$ of the point $\boldsymbol{x}_{I}$ using a top-hat filter. In order to increase the support of the filter, the smoothing is also performed for the set of second neighbors $\mathcal{N}^{2}\left(\boldsymbol{x}_{I}\right)$, third neighbors $\mathcal{N}^{3}\left(\boldsymbol{x}_{I}\right)$, and fourth neighbors $\mathcal{N}^{4}\left(\boldsymbol{x}_{I}\right)$ (cf. Sect. 2.1). It is given by

$$
\overline{\boldsymbol{u}}^{k}\left(\boldsymbol{x}_{I}\right)=\frac{1}{\left|\mathcal{N}^{k}\left(\boldsymbol{x}_{I}\right)\right|} \sum_{\boldsymbol{x}_{J} \in \mathcal{N}^{k}\left(\boldsymbol{x}_{I}\right)} \boldsymbol{u}\left(\boldsymbol{x}_{I}\right),
$$

where $\left|\mathcal{N}^{k}\left(\boldsymbol{x}_{I}\right)\right|$ denotes the number of points in the neighborhood. This method has the disadvantage that the additional computational effort grows with $\left|\mathcal{N}^{k}\left(\boldsymbol{x}_{I}\right)\right|$; therefore, in the second variant the velocity field is smoothed recursively

$$
\overline{\boldsymbol{u}}^{k}\left(\boldsymbol{x}_{I}\right)=\frac{1}{\left|\mathcal{N}^{1}\left(\boldsymbol{x}_{I}\right)\right|} \sum_{\boldsymbol{x}_{J} \in \mathcal{N}^{1}\left(\boldsymbol{x}_{I}\right)} \overline{\boldsymbol{u}}^{k-1}\left(\boldsymbol{x}_{I}\right),
$$

with $\overline{\boldsymbol{u}}^{0}\left(\boldsymbol{x}_{I}\right)=\boldsymbol{u}\left(\boldsymbol{x}_{I}\right)$. This formulation results in a bell-shaped filter function, where the influence of $\boldsymbol{u}\left(\boldsymbol{x}_{J}\right)$ decreases with growing distance from $\boldsymbol{x}_{I}$. 
Another idea is to use the approximate deconvolution approach by Chow et al. [11] and Stolz et al. [80], to obtain another reconstruction method for the sub-grid velocity. An approximation of the inverse filter function $\mathcal{G}^{-1}$ is constructed using the consecutively filtered velocities $\overline{\boldsymbol{u}}^{k}$. With the unfiltered velocity given by $\boldsymbol{u}_{\mathrm{DNS}}=\mathcal{G}^{-1} * \boldsymbol{u}_{\mathrm{LES}}$, where $\boldsymbol{u}_{\mathrm{LES}}$ is given by the hybrid RANS/LES solution, and the decomposition $\boldsymbol{u}_{\mathrm{DNS}}=\boldsymbol{u}_{\mathrm{LES}}+\boldsymbol{u}_{\mathrm{sgs}}$, the sub-grid scale velocity can be computed as

$$
\boldsymbol{u}_{\mathrm{sgs}}=\left(1-\mathcal{G}^{-1}\right) * \boldsymbol{u} .
$$

In Reuß et al. [D] (see Appx.) the described options to compute $\boldsymbol{u}_{\text {sgs }}$ are assessed for the test case of isotropic turbulence. Moreover, the flow over a backward-facing step is considered, as this case contains the relevant flow phenomena of a shear layer near a viscous wall, while the limited complexity allows for a global grid refinement. Additionally, the sensor $S_{1}$ based on the modeled sub-grid velocity $\overline{\boldsymbol{u}}^{4}\left(\boldsymbol{x}_{I}\right)$ with recursive filtering is selected as input for a local grid adaptation in the backward-facing step case. Some results are shown in Sect. 4.3.

\subsection{Algebraic Delayed DES}

Besides the grid resolution, another source of uncertainty arises from the behavior of the hybrid RANS/LES model. The algebraic sensors in the ADDES are designed to minimize these uncertainties.

To calculate the sensor values, the boundarylayer velocity profiles are evaluated along approximate wall-normal lines. The principle is depicted in Fig. 3.6. Starting point of the line search is the wall point $\boldsymbol{x}_{w}$, and the search ends either when no more valid points are found, or when the line reaches a prescribed length. The line that starts at the wall point $\boldsymbol{x}_{w}$ is denoted by $\mathcal{L}\left(\boldsymbol{x}_{w}\right)$.

During the search the surface-normal vector $\boldsymbol{n}$ of the boundary face is passed along the line. Let $\boldsymbol{x}_{u}$ be the last point added to $\mathcal{L}\left(\boldsymbol{x}_{w}\right)$, i.e. the

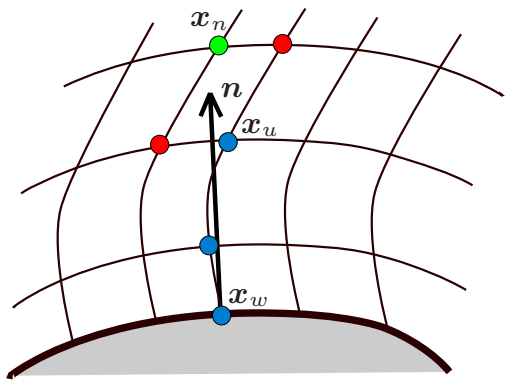

Fig. 3.6: Wall-normal line detection. uppermost point in the wall line. The next point is chosen from the set of the second neighbors $\boldsymbol{x}_{I} \in \mathcal{N}^{2}\left(\boldsymbol{x}_{u}\right)$. The angle $\theta$ is enclosed between $\boldsymbol{n}$ and the connection vector $\boldsymbol{x}_{I}-\boldsymbol{x}_{w}$. The next point $\boldsymbol{x}_{n}$ is determined by the minimal angle $\theta$

$$
\boldsymbol{x}_{n}=\max _{\boldsymbol{x}_{I} \in \mathcal{N}^{2}\left(\boldsymbol{x}_{u}\right)}\left(\frac{\boldsymbol{n} \cdot\left(\boldsymbol{x}_{I}-\boldsymbol{x}_{w}\right)}{\|\boldsymbol{n}\|\left\|\left(\boldsymbol{x}_{I}-\boldsymbol{x}_{w}\right)\right\|}\right)
$$

One important property of the line search is that the points in one line are not required to be direct neighbors in the grid. This provides maximal flexibility of the search algorithm in the case of curvilinear grids. In the example in Fig. 3.6, the gap between $\boldsymbol{x}_{u}$ and $\boldsymbol{x}_{n}$ enables the wall-normal progression of the line. 


\subsubsection{Parallel evaluation of wall-normal line data}

For an efficient evaluation of the boundary-layer profiles, the wall-normal lines are stored in a special parallelized data structure. In parallel computations, the search algorithm is able to detect the lines even if they are split by partition boundaries.

To this end, the line search for the wall point $\boldsymbol{x}_{w}$ is also stopped if a point $\boldsymbol{x}_{p}$ at a partition boundary is reached. The coordinates $\boldsymbol{x}_{w}$ and the surface-normal vector $\boldsymbol{n}$ of the corresponding wall point are communicated to the partition-boundary point $\boldsymbol{x}_{p}^{\prime}$ in the adjacent partition. Then the line search is restarted at the partition-boundary point $\boldsymbol{x}_{p}^{\prime}$ in the adjacent partition. The algorithm can follow the lines, even if they pass through several partitions. In Fig. 3.7 the lines in a partitioned grid are shown, where the partitions are indicated by different colors. The black lines start at wall points; the red lines, starting at partition boundaries, continue the black lines.

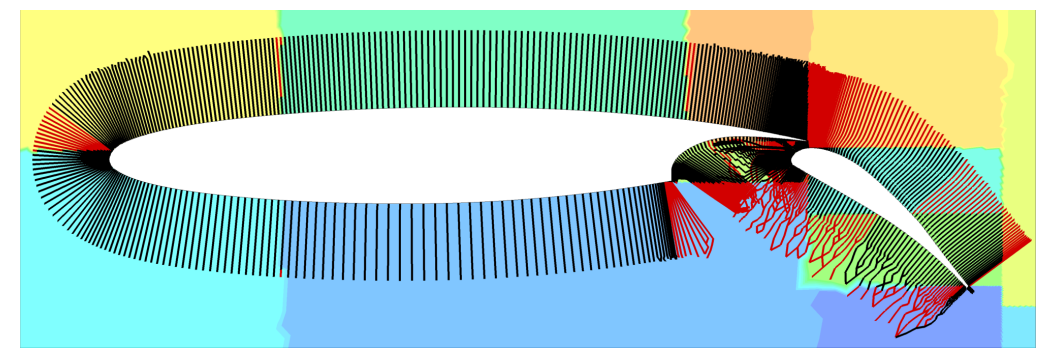

Fig. 3.7: Wall lines crossing the boundaries of the partitioned computational domain around a two-element airfoil.

The parallel evaluation of the boundary-layer profile data is a non-local operation, which means that additional communication routines must be provided. Two communication directions have been implemented in the ADDES. In the first direction, called the field-point-to-wall-point communication, the points in a line $\boldsymbol{x}_{I} \in \mathcal{L}\left(\boldsymbol{x}_{w}\right)$ send data to the starting wall point of the line $\boldsymbol{x}_{w}$. In the second direction, called the wall-point-to-field-point communication, the boundary points $\boldsymbol{x}_{w} \in \mathcal{X}_{\text {wall }}$ send data to the field points $\boldsymbol{x}_{I}$ with $f_{\mathrm{nwp}}\left(\boldsymbol{x}_{I}\right)=\boldsymbol{x}_{w}$, i.e. to all points that have $\boldsymbol{x}_{w}$ as their nearest wall point.

\subsubsection{Algebraic boundary-layer sensors}

The algebraic sensors control the switching between the RANS mode and the LES mode to fulfill the requirements in Sect. 3.1. Through the evaluation of the sensors, the following flow states are discerned for each point $\boldsymbol{x}_{I} \in \mathcal{X}$ :

(S1): The point $\boldsymbol{x}_{I}$ is located within the boundary layer.

(S2): The point $\boldsymbol{x}_{I}$ is located within a separated region.

(S3): The point $\boldsymbol{x}_{I}$ is treated in wall-modeled LES mode.

The sensor values are obtained by evaluating the boundary-layer velocity profiles. In the first step the tangential velocity ${ }^{1} u_{\|}=\left\|\boldsymbol{u}_{\|}\right\|$is computed for all points in

\footnotetext{
${ }^{1}$ The tangential velocity vector is computed as $\boldsymbol{u}_{\|}=\boldsymbol{u}-(\boldsymbol{u} \cdot \boldsymbol{n}) \boldsymbol{u}$, with the unit surface normal vector $\boldsymbol{n}$ in the corresponding wall point.
} 
the line $\boldsymbol{x}_{I} \in \mathcal{L}\left(\boldsymbol{x}_{w}\right)$ to obtain the sensor value for each boundary point $\boldsymbol{x}_{w}$. The points in the line $\mathcal{L}\left(\boldsymbol{x}_{w}\right)$ send the necessary data through the field-point-to-wallpoint communication. Afterwards, the wall-point-to-field-point communication is employed to distribute the sensor values to all field points where they are used to adjust the shielding function of the hybrid model.
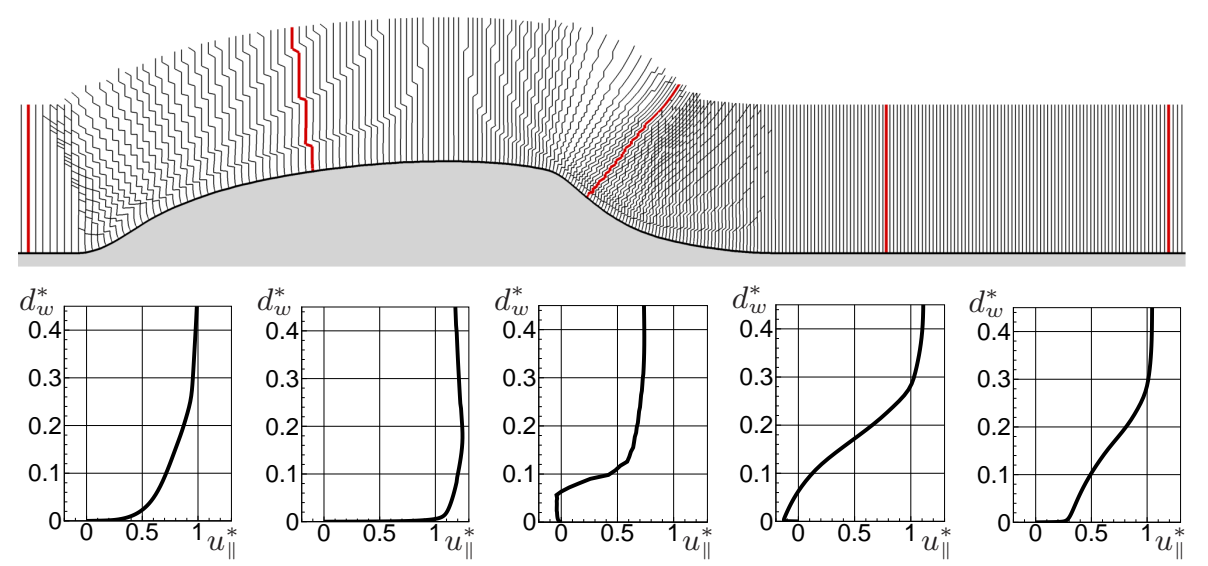

Fig. 3.8: Wall-normal lines and velocity profiles for the flow over a wall-mounted hump, with $d_{w}^{*}=d_{w} / h$ and $u_{\|}^{*}=u_{\|} / u_{\infty}$.

In the example in Fig. 3.8, the wall-normal lines for the wall-mounted hump and the tangential velocity profiles that correspond to the thick red lines are shown. For a detailed test-case description see Sect. 4.3.

For the sensor (S1) the boundary-layer thickness ${ }^{2} \delta$ of the nearest wall point $\boldsymbol{x}_{w}=f_{\text {nwp }}\left(\boldsymbol{x}_{I}\right)$ is needed in each point $\boldsymbol{x}_{I}$. If the wall distance is smaller than the boundary-layer thickness, $d_{w}\left(\boldsymbol{x}_{I}\right)<\delta\left(\boldsymbol{x}_{w}\right)$, the point is located inside in the boundary layer. The following choices are available to compute $\delta$ :

$\boldsymbol{\delta}_{\mathbf{9 9}}$ : The boundary-layer thickness $\delta$ of a wall point $\boldsymbol{x}_{w}$ is given by

$$
\delta=\min \left\{d_{w}\left(\boldsymbol{x}_{I}\right): \boldsymbol{x}_{I} \in \mathcal{L}\left(\boldsymbol{x}_{w}\right) \wedge\left\|\boldsymbol{u}_{\|}\left(\boldsymbol{x}_{I}\right)\right\| \geq 0.99 u_{\delta}\left(\boldsymbol{x}_{w}\right)\right\} .
$$

Since in flows with pressure gradients $u_{\delta}$ does not necessarily correspond to the global free-stream velocity, it must be approximated locally. With the compressible Bernoulli equation, the velocities $u_{1}=\left\|\boldsymbol{u}\left(\boldsymbol{x}_{1}\right)\right\|$ and $u_{2}=\left\|\boldsymbol{u}\left(\boldsymbol{x}_{2}\right)\right\|$ as well as the speeds of sound $a_{1}$ and $a_{2}$ in the two points $\boldsymbol{x}_{1}$ and $\boldsymbol{x}_{2}$ are related by

$$
\frac{\gamma-1}{2} u_{1}^{2}+a_{1}^{2}=\frac{\gamma-1}{2} u_{2}^{2}+a_{2}^{2} .
$$

Let point $\boldsymbol{x}_{1}$ be located at the wall, $\boldsymbol{x}_{1}=\boldsymbol{x}_{w}$ with $u_{w}=0$, and point $\boldsymbol{x}_{2}$ at the edge of the boundary layer, $\boldsymbol{x}_{2}=\boldsymbol{x}_{\delta}$. Then an equation for the boundary-layer edge velocity $u_{\delta}$ can be obtained. Keeping point $\boldsymbol{x}_{1}$ and moving point $\boldsymbol{x}_{2}$ to the farfield, $\boldsymbol{x}_{2}=\boldsymbol{x}_{\infty}$ with the free-stream velocity, an expression for the speed of sound at the wall is obtained. This expression can be substituted in the

\footnotetext{
${ }^{2}$ The boundary-layer thickness $\delta=\delta_{99}$ is defined as the minimal wall-distance where $u_{\|}$reaches $99 \%$ of the velocity at the boundary-layer edge $u_{\delta}$. In flows without any pressure gradient, $u_{\delta}$ corresponds to the global free-stream velocity $u_{\infty}$.
} 
equation for $u_{\delta}$. Using the relations $a=\sqrt{\gamma R T}, M a=\frac{u}{a}$, and $\frac{T_{\delta}}{T_{\infty}}=\left(\frac{p_{\delta}}{p_{\infty}}\right)^{\frac{\gamma-1}{\gamma}}$, the edge velocity $u_{\delta}$ can be estimated as

$$
u_{\delta}=u_{\infty} \sqrt{1+\frac{1-\left(\frac{p_{\delta}}{p_{\infty}}\right)^{\frac{\gamma-1}{\gamma}}}{\frac{\gamma-1}{2} M a_{\infty}^{2}}} .
$$

$\Xi_{\max }$ : In flows with a favorable pressure gradient, $u_{\|}$continues to grow outside the boundary-layer. For those cases an alternative detection of the boundary-layer thickness is available, according to Stock and Haase [79]. Following their approach, for each point $\boldsymbol{x}_{I} \in \mathcal{L}\left(\boldsymbol{x}_{w}\right)$ the value of $\Xi$ is evaluated based on the slope of the velocity profile as

$$
\Xi=y \frac{\partial u_{\|}}{\partial y},
$$

where $y$ corresponds to the wall distance $d_{w}$. Then the set of the local maxima $\Xi_{\max }$ is determined along the line, and the wall distance of the first local maximum is denoted as $d_{\max }=\min \left\{d_{w}\left(\boldsymbol{x}_{I}\right): \boldsymbol{x}_{I} \in \mathcal{L}\left(\boldsymbol{x}_{w}\right) \wedge \Xi\left(\boldsymbol{x}_{I}\right) \in \Xi_{\max }\right\}$. With a constant factor $C_{\Xi}=1.548$, recalibrated by Knopp and Probst [34], the boundary-layer thickness is given by

$$
\delta=C_{\Xi} d_{\max } .
$$

If according to sensor (S1) for a point $d_{w}\left(\boldsymbol{x}_{I}\right)<\delta\left(\boldsymbol{x}_{w}\right)$ holds, the shielding function of the hybrid RANS/LES model is modified to activate the RANS mode. In Fig. 3.9 this region is shaded in red for the wall-mounted hump. If only the sensor (S1) was used, the whole recirculation region in the wake of the hump would be treated in the RANS mode.

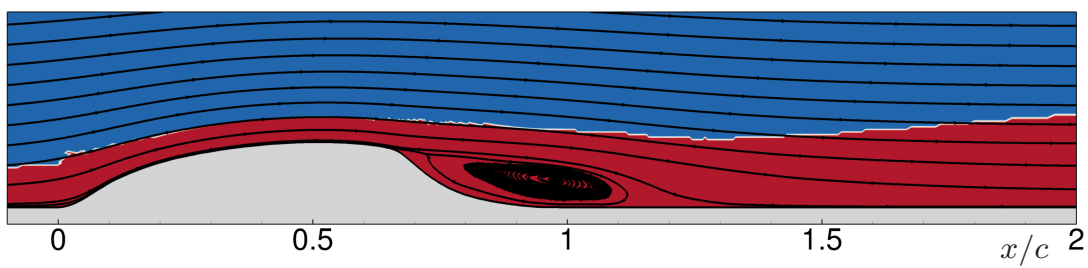

Fig. 3.9: Boundary layer in the wall-mounted hump flow as detected by the algebraic sensor (S1).

Therefore, the second sensor (S2) is designed to detect separated flow regions. According to White [88], the shape factor $H_{12}$ grows with growing values of the pressure gradient, where high values of $H_{12}$ indicate a strong adverse pressure gradient. In turbulent boundary layers, flow separation occurs if $H_{12}$ exceeds an empirical critical value of about 2.76, see Castillo et al. [6]. The shape factor is defined as $H_{12}=\delta_{1} / \delta_{2}$, where $\delta_{1}$ is the displacement thickness and $\delta_{2}$ is the momentum thickness

$$
\delta_{1}=\int_{0}^{\infty}\left(1-\frac{\rho\left(y^{\prime}\right) u_{\|}\left(y^{\prime}\right)}{\rho_{0} u_{0}}\right) \mathrm{d} y^{\prime}, \quad \delta_{2}=\int_{0}^{\infty} \frac{\rho\left(y^{\prime}\right) u_{\|}\left(y^{\prime}\right)}{\rho_{0} u_{0}}\left(1-\frac{u_{\|}\left(y^{\prime}\right)}{u_{0}}\right) \mathrm{d} y^{\prime} .
$$

The values are integrated along the line $\mathcal{L}\left(\boldsymbol{x}_{w}\right)$, and the upper integration boundary is replaced by the boundary-layer thickness that is obtained using the sensor (S1). In 
the ADDES the critical value that corresponds to the separation location has been calibrated for each turbulence model, resulting in $H_{12 \text {, crit }}=2.4$ for the SA model, see Knopp and Probst [34], $H_{12 \text {,crit }}=3.3$ for the SST model, see Geurts and Probst [21], and $H_{12 \text {,crit }}=3.2$ for the JHh-v2 RSM model, see Probst et al. [63]. In Fig. 3.10 the development of $H_{12}$ from a SA-RANS simulation of the wall-mounted hump is shown. The red line indicates $H_{12 \text {, crit }}=2.4$.

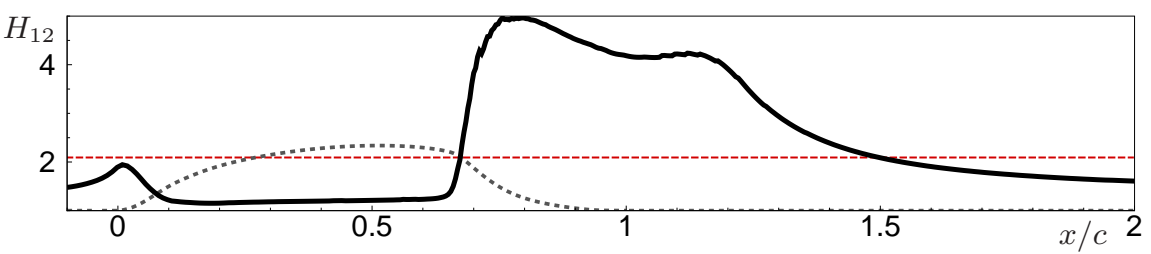

Fig. 3.10: Shape factor distribution along the wall-mounted hump.

If according to sensor (S2) a point is located in a region of separated flow, the shielding functions are modified again to activate the LES mode in this region. In Fig. 3.11 this region is shaded in red for the wall-mounted hump. If only the sensors (S1) and (S2) were used, the flow downstream of the reattachment point would be treated in the RANS mode.

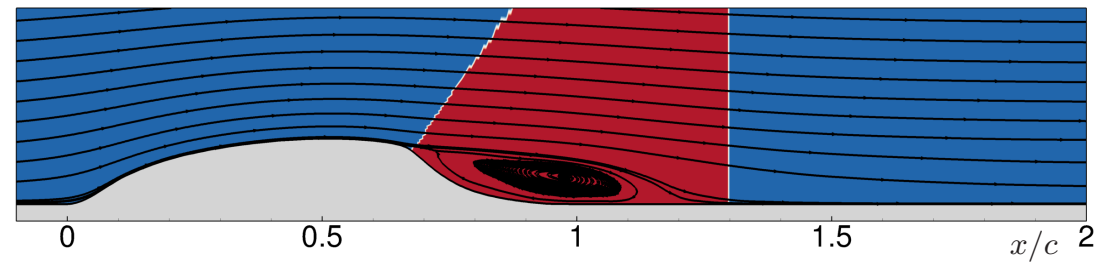

Fig. 3.11: Separated region in the wall-mounted hump flow as detected by the algebraic sensor (S2).

Therefore, the sensor (S3) is designed to detect the wall-modeled LES mode. It follows the IDDES approach, where the function $\tilde{f}_{d}$ in Eq. (1.54) is based on the values of $f_{d t}$ in Eq. (1.53) and $f_{B}$ in Eq. (1.51). A value of $\left(1-f_{d t}\right)=1$ activates the RANS model in the DDES mode of the IDDES, while $f_{B}=1$ ensures a small RANS region in the inner region of the boundary layer if it is treated in wall-modeled LES mode. The sensor compares the values of $f_{d t}$ and $f_{B}$ along the wall-lines. The wall distance where $\left(1-f_{d t}\right)$ drops below one is denoted as $\delta_{d t}$, while $\delta_{B}$ correspondingly denotes the distance where $f_{B}$ drops below one. If $\delta_{d t}<C_{\mathrm{wm}} \cdot \delta_{B}$, the boundary layer is considered to be in wall-modeled LES mode, and the shielding function is modified accordingly, i.e. $f_{d t}$ is set to zero. A calibration of the empirical weighting factor $C_{\mathrm{wm}}$ using the wall-mounted hump flow, yields $C_{\mathrm{wm}}=1.8$.

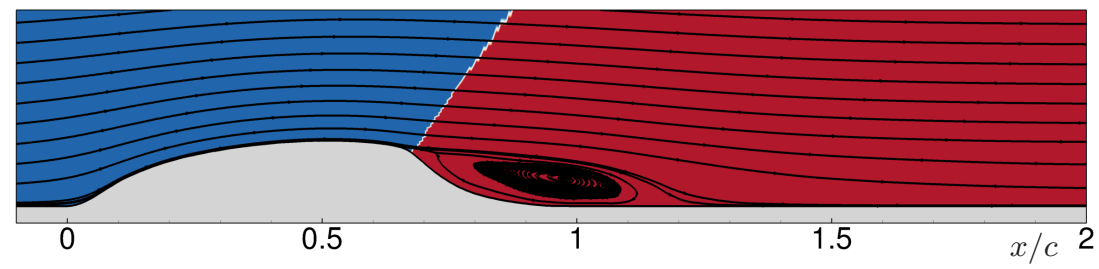

Fig. 3.12: Wall-modeled LES region in the wall-mounted hump flow as detected by the algebraic sensor (S3). 


\subsubsection{Improved LES filter width in the ADDES}

In the definition of the LES length scale in Eq. (1.40), the LES filter width $\Delta_{\text {LES }}$ is determined by the grid-cell size. To find a suited measure for $\Delta_{\mathrm{LES}}$, the cells types that can be typically found in grids for hybrid RANS/LES simulations are categorized following the nomenclature by Mockett et al. [49].

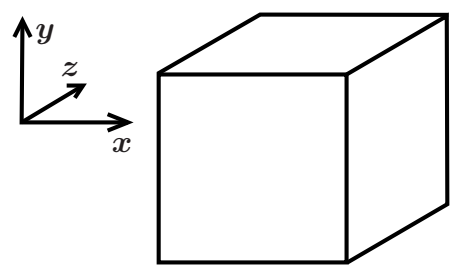

(a) box-shaped cell

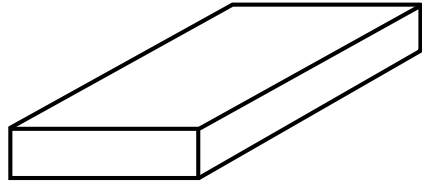

(b) book-shaped cell

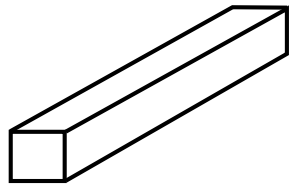

(c) pencil-shaped cell

Fig. 3.13: Categorization of grid-cell types depending on the aspect ratio of the edges.

Depending on the aspect ratio of the edge lengths $\Delta_{x}$ (stream-wise), $\Delta_{y}$ (wallnormal), and $\Delta_{z}$ (span-wise), three different cell types are depicted in Fig. 3.13: (a) box-shaped cells with $\Delta_{x} \approx \Delta_{y} \approx \Delta_{z}$, (b) book-shaped cells with $\Delta_{y}<\Delta_{x}<\Delta_{z}$, and (c) pencil-shaped cells with $\Delta_{x} \approx \Delta_{y} \ll \Delta_{z}$. LES regions are typically discretized with isotropic box-shaped cells; therefore, in LES models the filter width $\Delta_{\text {LES }}$ can for example be defined through the cell volume, see e.g. Fröhlich [19]

$$
\Delta_{\mathrm{vol}}=\sqrt[3]{\Delta_{x} \cdot \Delta_{y} \cdot \Delta_{z}}=\sqrt[3]{\left|V_{I}\right|},
$$

where the second expression is used in the dual-grid approach. The book-shaped cells are typically found in boundary-layer regions if the grid resolution is suited for a RANS model. To obtain a conservative formulation for hybrid RANS/LES grids with mixed box-shaped and book-shaped cells, Spalart et al. [77] propose to use the maximum edge length for the LES filter in the original DES

$$
\Delta_{\max }=\max \left(\Delta_{x}, \Delta_{y}, \Delta_{z}\right)=\max _{\boldsymbol{x}_{J} \in \mathcal{N}\left(\boldsymbol{x}_{I}\right)}\left|\boldsymbol{x}_{I}-\boldsymbol{x}_{J}\right| .
$$

For isotropic box-shaped cells the values coincide, i.e. $\Delta_{\mathrm{vol}}=\Delta_{\max }$.

As described in Sect. 3.2.1, a three-dimensional grid can be generated by stacking two-dimensional grids in the span-wise direction with equidistant span-wise cells. Near discontinuities in the surface slope this procedure typically leads to pencilshaped cells as depicted in Fig. 3.14 for the backward-facing step. For these cells the filter width $\Delta_{\max }$ yields a very conservative estimation. As a consequence the hybrid model switches from RANS to LES very slowly, leading to a large grey area where the development of resolved turbulent structures is considerably delayed.

To prevent this modeling uncertainty, Chauvet et al. [9] propose an LES filter width that is adapted to the local flow. The main rotation axis of the vortices is given by the vorticity vector $\boldsymbol{\omega}=\nabla \times \boldsymbol{u}$, and only the cell dimensions perpendicular to the rotation axis are taken into account. With the normalized vorticity vector, denoted by $\boldsymbol{N}=\left(N_{x}, N_{y}, N_{z}\right)^{t}$, the filter width in regular structured cells is defined as

$$
\Delta_{\omega, \text { orig }}=\sqrt{N_{x}^{2} \Delta_{y} \Delta_{z}+N_{y}^{2} \Delta_{x} \Delta_{z+} N_{z}^{2} \Delta_{x} \Delta_{y}} .
$$

Deck [13] generalizes this formulation for unstructured tetrahedral cells. Another approach that combines the idea of $\Delta_{\max }$ and $\Delta_{\omega}$ is proposed by Mockett et al. [49]. 


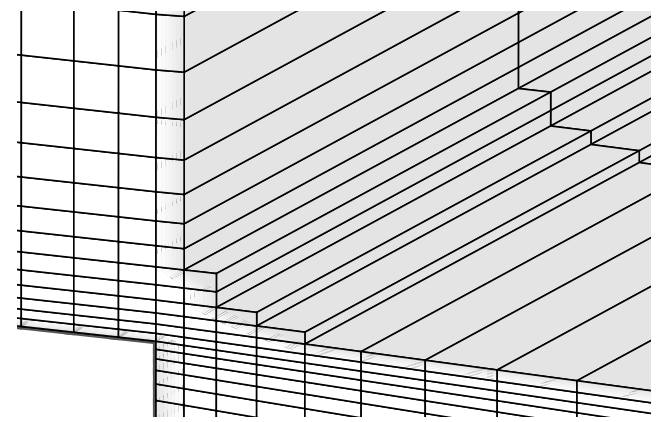

Fig. 3.14: Cut through the backward-facing step grid: pencil-shaped cells at the sharp edge.

Since both, the formulation in Eq. (3.17) and its generalization by Deck [13], are difficult to adopt for the unstructured dual cell approach of TAU, a purely facebased approximation of the vorticity-based filter width was developed in the present thesis, given by

$$
\Delta_{\omega}=\sqrt{\frac{1}{2} \sum_{\boldsymbol{x}_{J} \in \mathcal{N}\left(\boldsymbol{x}_{I}\right)} \boldsymbol{N}_{I} \cdot \boldsymbol{n}_{I J}}
$$

where $\boldsymbol{n}_{I J}$ is the area-weighted face-normal vector. This form can be efficiently evaluated in each physical time step of a TAU simulation. For its combination with the IDDES, consider the definition of the filter width in IDDES according to Travin et al. [85]

$$
\Delta_{\mathrm{IDDES}}=\min \left(\max \left(C_{w} d_{w}, C_{w} \Delta_{\max }, h_{w n}\right), \Delta_{\max }\right),
$$

with the wall-normal cell size $h_{w n}$. A lower limit of this filter width is given by $C_{w} d_{w}$, with $C_{w}=0.15$. Between this lower limit and $\Delta_{\max }$ the shape of the curve depends on the growth rate of the cells in the wall-normal direction: if it is larger than $1.15, h_{w n}$ grows faster than the weighted wall distance $C_{w} d_{w}$. This adjustment is primarily effective within the boundary layer and is calibrated for the wall-modeled LES mode.

In the combination of Eq. (3.19) and the vorticity-based LES filter, the wall-modeled LES mode should be unaltered; therefore, only the second occurrence of $\Delta_{\max }$ is substituted, and the modified IDDES filter width reads

$$
\Delta_{\text {IDDES }}=\min \left(\max \left(C_{w} d_{w}, C_{w} \Delta_{\max }, h_{w n}\right), \Delta_{\omega}\right) .
$$

In Probst and Reuß [G] this formulation is applied to simulate a turbulent channel flow, and it is shown that the wall-modeled LES capability of the IDDES can be retained. Some results are summarized in Sect. 4.2, together with new results that consider higher Reynolds numbers. Results of precursor studies of the flow over a backward-facing step are shown in Sect. 4.3, where the IDDES filter widths based on $\Delta_{\omega}$ and $\Delta_{\max }$, Eqs. (3.19) and (3.20), are compared. Since $\Delta_{\omega}$ is found to reduce the grey area significantly, it is used in Reuß et al. [D] (see Appx.) as well.

\subsubsection{Synthetic turbulence at the RANS-to-LES interface}

The sensor (S2) enables a precise activation of the LES mode inside the boundary layer at the location of flow separation, as shown in Fig. 3.11 for the flow over a 
wall-mounted hump. At the interface where the model switches from RANS to LES it is subjected to the grey-area problem. Besides the improved LES filter width $\Delta_{\omega}$, further means are desirable to accelerate the RANS-LES transition in rather stable weakly separated flows.

To force the generation of resolved turbulence in the LES region, artificial disturbances can be fed into the flow as described in Eq. (1.44) for the embedded LES approaches. A simple method is the stochastic forcing by Kok and van der Ven [37] where the eddy viscosity is multiplied by a normally distributed random variable $\phi$, with zero mean and unit variance

$$
\mu_{t, \mathrm{stoch}}=\phi \mu_{t} .
$$

The value of $\phi$ is recomputed for each grid point in each physical time step. In the case of a non-separated turbulent channel flow, such "white-noise" fluctuations are damped rapidly as shown by Pamiès et al. [57]; however, in less stable flow situations stochastic forcing can be useful. For example, it is successfully applied in Reuß et al. [F] (see Appx.) to destabilize the free shear layer in the wake of a NACA-0021 airfoil. Some of the results are summarized in Sect. 4.6.

In order to introduce more realistic artificial structures, it is necessary to maintain the spatial and temporal coherence characteristics of realistic turbulence. In the synthetic turbulence generator by Adamian and Travin [3], the matrix $\boldsymbol{A}$ that is obtained by a Cholesky decomposition of the Reynolds stress tensor, $\boldsymbol{\tau}_{\mathrm{RANS}}=\boldsymbol{A}^{T} \boldsymbol{A}$, and the auxiliary velocity field $v_{i}^{\prime}$ are used to define synthetic velocity fluctuations as

$$
\boldsymbol{u}^{\prime}=\boldsymbol{A} \boldsymbol{v}^{\prime} \quad \text { with } \quad\left\langle\boldsymbol{v}^{\prime}\right\rangle=\mathbf{0} \quad \text { and } \quad\left\langle\boldsymbol{v}^{\prime} \otimes \boldsymbol{v}^{\prime}\right\rangle=\boldsymbol{I},
$$

with the unity matrix $\boldsymbol{I}$. By this construction the second moment tensor of the synthetic fluctuations equals the Reynolds stress tensor, $\rho\left\langle u_{i}^{\prime} u_{j}^{\prime}\right\rangle=\tau_{\text {RANS }, i j}$. The auxiliary velocity fluctuations are generated by superposing $N$ weighted Fourier modes, with the wave number $k$ and the normalized amplitude $q$. The global time scale $\tau$ is obtained from the length scale of the turbulence model and a characteristic velocity. Several random values are used to generate the stochastic Fourier modes. The random wave vector $\boldsymbol{d}$ is uniformly distributed over the unit sphere, and the random vector $\boldsymbol{\sigma}$ is defined perpendicular to $\boldsymbol{d}$, such that the angle that is enclosed between $\boldsymbol{d}$ and $\boldsymbol{\sigma}$ is uniformly distributed. The phase $\phi \in[0,2 \pi)$ is an uniformly distributed random number and the frequency $s$ is a random number with Gaussian distribution. With this, the synthetic velocity fluctuations are given by

$$
\boldsymbol{v}^{\prime}(\boldsymbol{x}, t)=\sqrt{6} \sum_{n=0}^{N} \sqrt{q^{n}}\left(\boldsymbol{\sigma}^{n} \cos \left(k^{n} \boldsymbol{d}^{n} \cdot \boldsymbol{x}+\phi^{n}+s^{n} \frac{t}{\tau}\right)\right) .
$$

François and Radespiel [17] implemented this approach in TAU. They extended it to a divergence-free formulation with $\frac{\partial v_{i}^{\prime}}{\partial x_{i}}=0$, and they accelerated the development of realistic turbulence substantially, by introducing the artificial fluctuations within a volume, rather than in a plane. The algebraic sensors of the ADDES allow the automatic detection of the forcing regions and the regions of the reference points. Part of the present thesis was to provide a flexible infrastructure, so that the synthetic turbulence can be introduced in several locations.

In François et al. [J] further applications of the method are presented. In Sect. 4.2 and Sect. 4.5 the synthetic turbulence is utilized in the present thesis. As an SSTbased approach is used, the Reynolds stress tensor is not directly available, so that the approximate Reynolds stress tensor is computed from the Boussinesq assumption. 


\subsubsection{Sponge layer at the LES-to-RANS interface}

In DDES and IDDES simulations of the DLR F15 three-element airfoil in Reuß et al. $[\mathrm{B}]$ (see Appx.) and Reuß et al. $[\mathrm{C}]$ (see Appx.), resolved structures from the turbulent shear layer in the slat wake penetrate into the shielded boundary layer on the main wing, see Sect. 4.1. These structures deteriorate the flow prediction downstream, and as a consequence the separation on the flap is suppressed. Even if the RANS mode is enforced on the main wing, the resolved structures are partly maintained. A similar observation was made in precursor simulations for the flow over the backward-facing step, which are presented in Sect. 4.3: resolved structures from the recirculation region are transported into the attached boundary layer downstream of the reattachment point. Only the wall-modeled LES mode of IDDES is found to correctly predict the flow in this region, whereas the DDES result shows larger deviations from the experimental data. Therefore, in Reuß et al. [D] (see Appx.) the IDDES approach is used. However, if the required resolution for a wall-modeled LES can not be provided, the RANS mode is the only option.

In this case additional effort is necessary to prevent the negative influence of the resolved turbulent structures on the RANS solution. For this purpose, source terms have been tested in combination with the ADDES. They are added to the transport equations in the RANS region, in order to damp the resolved fluctuations and transfer the extracted kinetic energy into modeled turbulent kinetic energy. In the momentum equation (1.20), the source term is given by the difference between the target velocity $\boldsymbol{u}_{\text {target }}$ and the current velocity $\boldsymbol{u}$

$$
\frac{\Delta \boldsymbol{u}}{\tau}, \quad \text { with } \quad \Delta \boldsymbol{u}=\boldsymbol{u}_{\text {target }}-\boldsymbol{u} \quad \text { and } \quad \tau=1000 \frac{\mu}{\rho\|\boldsymbol{u}\|^{2}},
$$

where is $\tau$ the turbulent time scale. In the $k$-equation Eq. (1.23) of the SST turbulence model, the source term is given by the difference between the target turbulent kinetic energy $k_{\text {target }}$ and the current modeled turbulent kinetic energy $k$

$$
\frac{\Delta k}{\tau}, \quad \text { with } \quad \Delta k=k_{\text {target }}-k .
$$

The local value of the mean velocity is used as the target value for the control mechanism, $\boldsymbol{u}_{\text {target }}=\langle\boldsymbol{u}\rangle$, and the target turbulent kinetic energy is obtained as the sum of the mean modeled turbulent kinetic energy and the mean velocity fluctuations $k_{\text {target }}=\langle k\rangle+\left\langle\boldsymbol{u}^{\prime 2}\right\rangle$. The mean values are computed as running averages, according to Eq. (2.17).

In the target application of the present thesis the sponge terms play no role, but the implementation is assessed for a turbulent channel flow in Sect. 4.2. 



\section{Chapter 4}

\section{Application of the grid-adaptive algebraic hybrid RANS/LES model}

The developments in the present thesis aim to provide a hybrid RANS/LES simulation strategy for the numerical investigation of the stall behavior of high-lift airfoils under the influence of turbulent inflow. In the scenario under investigation the turbulent inflow is represented by a reproducible lateral vortex, which is generated by a pitching airfoil. The setup is depicted in Fig. 4.1. Experimental data for the validation of the simulation method are provided by Hahn et al. [24] and Klein et al. [32].

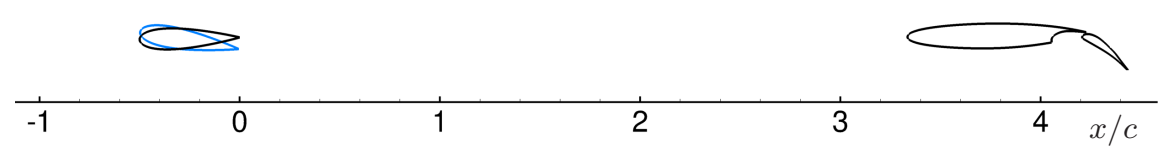

Fig. 4.1: Setup of the test case with the DLR F15 two-element airfoil and the vortexgenerator airfoil NACA-0021.

In a systematic approach, the problem is split into subtasks, which are described in detail in the next sections:

1) In Sect. 4.1 the initial state of the hybrid RANS/LES methods in TAU at the beginning of the work is documented using the example of the flow about a threeelement high-lift airfoil.

2) In Sect. 4.2-4.4 the grid-adaptive algebraic hybrid RANS/LES simulation strategy is applied to basic model test cases.

3) In Sect. 4.5 the findings from step 2) are reassessed for the two-element high-lift airfoil from the target application.

4) In Sect. 4.6 a suitable simulation strategy for the generation and transport of the lateral vortex from the target application is presented.

5) The interaction of the vortex with the two-element high-lift airfoil is investigated in Sect. 4.7.

In step 2) the new method is tested for generic test cases, which represent different regions of the flow about the high-lift airfoil. This approach isolates the problems to specifically investigate the effects of the model variations. Moreover, the computational effort for the basic test cases is considerably smaller. Fig. 4.2 illustrates the relations between the basic cases and the target application. In the picture a three-element airfoil is shown, whereas in the target application a two-element airfoil is considered. However, in both cases the attached boundary layer at the main wing 


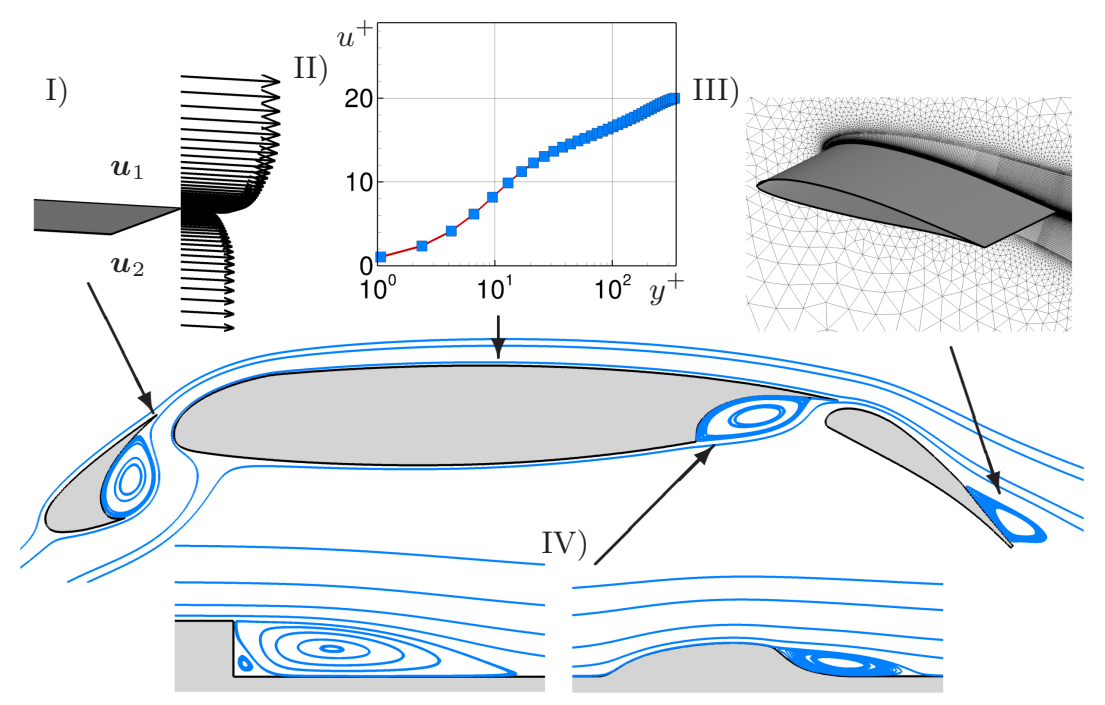

Fig. 4.2: Relevance of basic test cases for the flow about a high-lift airfoil.

is subjected to turbulent onflow: in Fig. 4.2 the turbulence is generated in the wake of the slat, while in the target application the turbulence is generated by the airfoil further upstream.

I) The flow downstream of the trailing edges of the slat and the main wing is represented by the plane shear layer, which is a very challenging test case, see e.g. Mockett et al. [49]. A similar situation is found downstream of the NACA0021 vortex-generator airfoil in the target application. In Reuß et al. $[\mathrm{F}]$ (see Appx.) a stochastic forcing was required to destabilize the wake flow, in order to excite the generation of resolved LES structures.

II) The turbulent slat wake impinges upon the attached boundary layer on the main wing element. To investigate the behavior of the hybrid RANS/LES models in this situation, the turbulent channel flow is a representative model case, which is addressed in Sect. 4.2.

III) To investigate the separation prediction on the flap, the flow about a singleelement airfoil is a suitable generic test case. As a matter of fact, the singleelement airfoil HGR01 is one of the examples, which motivated the developments in the present thesis, see Sect. 3.1. François and Radespiel [17] present hybrid RANS/LES results with synthetic turbulence for this case.

IV) The attached boundary layer on the lower side of the wing separates at the surface discontinuity at the cove edge and reattaches farther downstream in the cove. The flow over a backward-facing step represents this situation of a geometry-driven separation with reattachment, see Sect. 4.3. The wall-mounted hump, which is considered in Sect. 4.4, provides a similar flow topology, but in this case the flow separates over a smooth surface.

In the description of the test cases the following nomenclature of the directions is used: the $x$-coordinate denotes the stream-wise direction, $y$ corresponds to the wallnormal direction and the $z$-coordinate represents the span-wise direction. 


\subsection{Precursor simulations of a three-element airfoil}

In the initial phase of the present thesis, the basic hybrid RANS/LES models Delayed DES and Improved Delayed DES, as described in Sect. 1.3.3, were assessed for highlift applications. In Reuß et al. [B] (see Appx.) and Reuß et al. [C] (see Appx.) SA-based DDES and IDDES simulations for the DLR F15 three-element airfoil are compared. Here, the essential results and conclusions are briefly summarized.

The DLR F15 airfoil represents a cut section at 51.1\% span through the FNG wing ("Flügel neuer Generation"), designed by Dargel et al. [12]. Wild et al. [90] performed variations of the high-lift slat and flap to reduce the noise emission and provide experimental reference data for several angles of attack. As for high angles of attack the wind-tunnel side walls have a considerable effect on the flow in the center of the tunnel, the simulations consider a moderate incidence of $\alpha=6^{\circ}$.

\section{Computational setup}

The computational grid in the $x y$-plane is generated using the Centaur grid generator. An O-type grid topology is used to discretize the blunt trailing edges. The boundary layers are resolved with 45 layers of quadrilateral elements, while farther away from the wall flexible unstructured triangular elements are used.

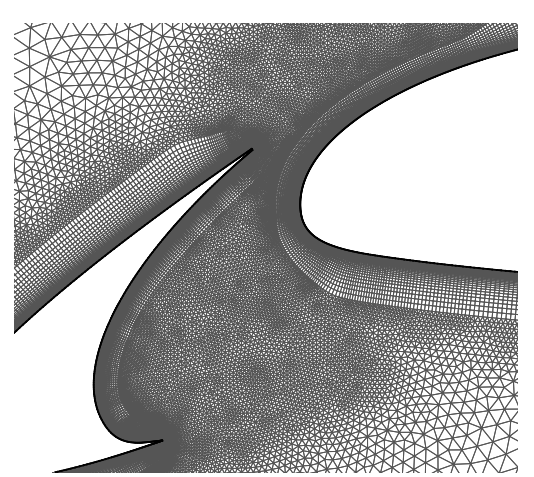

(a) Slat-cove region

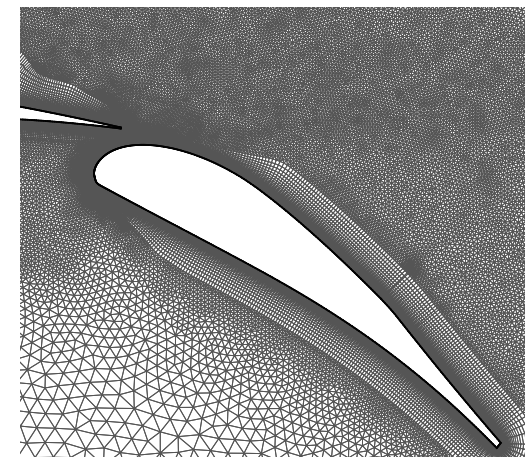

(b) Flap region

Fig. 4.3: Grid for the precursor hybrid RANS/LES simulations of the DLR F15 threeelement airfoil.

Fig. 4.3 shows the grid in the slat-cove region and the flap region. The computational domain in the span-wise direction extends over $9 \%$ of the retracted chord length $c$ and is resolved with 64 grid layers. In total, the grid contains $12.8 \times 10^{6}$ points. One convective time unit is resolved with 600 physical time steps. The Reynolds number of the flow is $R e=1.9 \times 10^{6}$, and the Mach number is $M a=0.15$.

In the experiments the position where the flow transitions from a laminar to a turbulent state is not fixed. In order to obtain comparable numerical results, the transition locations that are prescribed in the simulations are calibrated using experimental data. This procedure is described in Reuß et al. [B] (see Appx.). 


\section{Results}

In the initial DDES and IDDES simulations of the DLR F15 three-element airfoil, deficiencies of the model behavior in the confluent slat wake and the boundary layer on the main wing element were observed.

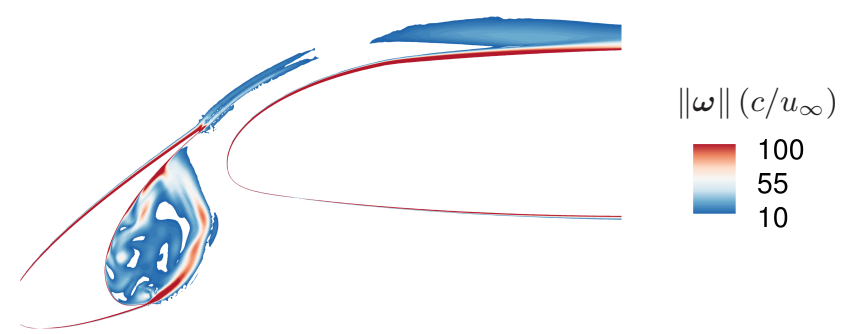

(a) SA-DDES

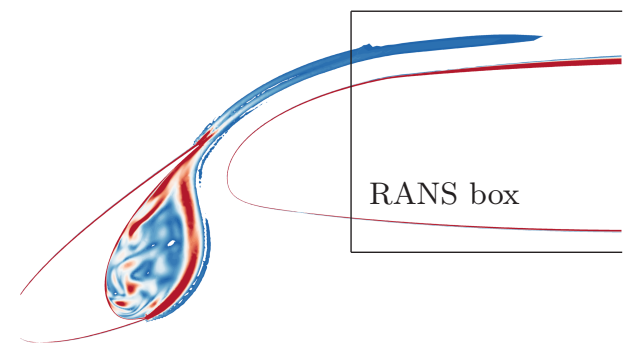

(b) SA-DDES with RANS box

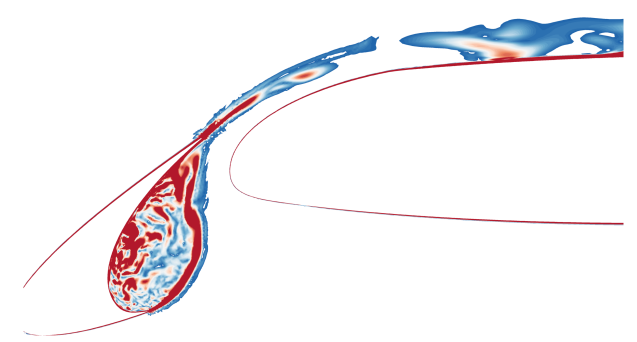

(c) SA-IDDES

Fig. 4.4: Vorticity snapshots in the confluent slat wake and the boundary layer from the initial hybrid RANS/LES simulations of the DLR F15 three-element airfoil.

Fig. 4 from Reuß et al. [B] (see Appx.) shows the resolved turbulent structures in the wake of the slat. A close-up of the slat region is reproduced in Fig. 4.4. With standard DDES in (a) the structures penetrate into the near-wall region on the main wing. Because the grid resolution is only suited for the RANS mode in this region, the resolved structures are not properly resolved. Those underresolved structures interfere with the modeled turbulence in the boundary layer. Two further approaches were tested to prevent this problem. On the one hand, the resolved structures are damped by enforcing the RANS mode in a modified, zonal DDES approach in (b). On the other hand, in (c) a refined grid is used to provide sufficient resolution to maintain the resolved structures in the wall-modeled LES mode of IDDES.

In all three simulations the influence of the slat wake on the boundary layer on the main wing is so strong, that the downstream behavior of the flow is massively disturbed. Therefore, with the tested approaches the separation on the flap is underpredicted or even completely suppressed.

According to later investigations, the following additional uncertainties can be identified in retrospect:

1. The numerical settings are not optimized for scale-resolving simulations.

2. No means are taken to enhance the development of resolved turbulent structures downstream of the trailing edges and thereby mitigate the grey-area problem.

3. The grid design is not optimal: 
(a) The O-type grid topology at the trailing edges leads to larger grid cells in the wake.

(b) The separated region above the flap is not fully covered by the structured part of the grid.

All these observations motivated the work in the present thesis to provide a reliable hybrid RANS/LES prediction method for high-lift airfoils. The numerical optimizations that are addressed by the first point are presented in Probst and Reuß [G], and they are also described in Sect. 2.3. The alternative LES filter definitions from Sect. 3.3.3 were developed to improve the second point. The third point is considered in the current best-practice guide lines for the grid generation in hybrid RANS/LES simulations, which are incorporated in the grid design for the two-element airfoil in Sect. 4.5 .

\subsection{Turbulent channel flow}

Because of its geometrical simplicity, the plane channel is well suited for the basic validation of simulation methods for wall-bounded flows. Moreover, DNS results are available as reference data for several Reynolds numbers. The fully-developed solution is self similar in the stream-wise and the span-wise direction. To reduce the computational effort, these symmetries can be exploited by prescribing periodic boundary conditions. Furthermore, the solution can be averaged in the directions of symmetry in a post-processing step. If periodic boundary conditions are used in the stream-wise direction, an additional uniform source term is necessary to drive the flow in the absence of a pressure gradient. This source term is given by

$$
f_{x}=\frac{u_{\text {bulk }}-u_{\text {bulk,DNS }}}{\tau} \quad \text { with } \quad \tau=1000 \frac{\mu}{\rho\left|u_{\text {bulk }}\right|^{2}}
$$

In this test case all length scales are given in terms of the half channel height $\delta$. Two Reynolds numbers are used to describe the flow and the accuracy of the numerical prediction: $R e_{\delta}$, based on the bulk velocity $u_{\text {bulk }}$, and $R e_{\tau}$, based on the friction velocity $u_{\tau}$, according to Eq. (1.29), which read

$$
R e_{\delta}=\frac{u_{\mathrm{bulk}} \delta}{\nu} \quad \text { with } \quad u_{\mathrm{bulk}}=\frac{1}{\delta} \int_{0}^{\delta}\left\langle u_{x}\right\rangle \mathrm{d} y, \quad \text { and } \quad R e_{\tau}=\frac{u_{\tau} \delta}{\nu}=\frac{\delta}{\delta_{\nu}}
$$

The viscous length scale $\delta_{\nu}$ is defined as

$$
\delta_{\nu}=\nu \sqrt{\frac{\rho}{\tau_{w}}}=\frac{\nu}{u_{\tau}} .
$$

To show the self-similarity of the velocity profiles, the non-dimensional velocity $u^{+}=u / u_{\tau}$ is plotted against the non-dimensional wall distance $y^{+}=d_{w} / \delta_{\nu}$.

\section{Computational setup}

The computational domain has a height of $2 \delta$ and a width of $3.2 \delta$. The length is $6.4 \delta$ for the simulations with stream-wise periodic boundary conditions, while it is extended to $51.2 \delta$ for the simulations with standard inflow and outflow boundary conditions. 
Three Reynolds numbers are considered for this case. DNS data are available for $R e_{\tau}=395$ from Moser et al. [52] and for $R e_{\tau}=4200$ from Lozano-Durán and Jiménez [43]. For the third Reynolds number, $R e_{\tau}=1100$, no DNS data are available. A grid resolution study is performed for the Reynolds number $R e_{\tau}=395$, using the grids $G_{1}, G_{2}$ and $G_{3}$. For the higher Reynolds numbers the wall-normal distribution of the grid points in the grid $G_{2}$ is adapted: for $R e_{\tau}=1100$ and $R e_{\tau}=4200$ the grids $G_{2}^{\prime}$ and $G_{2}^{\prime \prime}$ are used, respectively. The grid $G_{2}^{\prime \prime \prime}$ is used for the simulations with inflow and outflow boundary conditions at $R e_{\tau}=395$. To keep the resolution constant, the number of grid points in the stream-wise direction is adjusted. Details about the grids are given in Tab. 4.1.

\begin{tabular}{rrrrrrrrrr}
\hline Grid & $R e$ & $L_{x}$ & $\Delta_{x}^{+}$ & $\Delta_{y}^{+}$ & $\Delta_{z}^{+}$ & $N_{x}$ & $N_{y}$ & $N_{z}$ & $N_{\text {tot }}$ \\
\hline \hline$G_{1}$ & 395 & 6.4 & 31 & 1 & 13 & 81 & 97 & 97 & 762,000 \\
\hline$G_{2}$ & 395 & 6.4 & 41 & 0.8 & 19.5 & 62 & 65 & 65 & 262,000 \\
\hline$G_{3}$ & 395 & 6.4 & 120 & 0.8 & 37 & 21 & 65 & 43 & 46,000 \\
\hline$G_{2}^{\prime}$ & 1100 & 6.4 & 109 & 0.8 & 54 & 65 & 81 & 65 & 342,000 \\
\hline$G_{2}^{\prime \prime}$ & 4200 & 6.4 & 416 & 0.8 & 206 & 65 & 101 & 65 & 427,000 \\
\hline$G_{2}^{\prime \prime \prime}$ & 395 & 51.2 & 41 & 0.8 & 19.5 & 489 & 65 & 65 & $2,066,000$ \\
\hline
\end{tabular}

Tab. 4.1: Dimensions of the grids for the turbulent channel flow.

The simulations with stream-wise periodic boundary conditions are initialized with a solution, which contains artificially generated turbulent structures.

\section{Results}

In Probst and Reuß [G] and Probst et al. $[\mathrm{H}]$ the optimized numerical settings for low dissipation (LD) and the low-dissipation low-dispersion scheme (LD2), which are both described in Sect. 2.3, are utilized in wall-modeled LES simulations of the turbulent channel flow. It is shown that with both, LD and LD2, the results are considerably improved, compared to the standard scheme in TAU. The results of a grid study by Probst and Reuß [G] for the SA-based IDDES are shown in Fig. 4.5. The LD scheme is used, and the IDDES filter width is based on the LES filter $\Delta_{\max }$. The log-layer mismatch at the interface between RANS and LES, which can be observed for the solution on grid $G_{3}$, indicates that this grid is too coarse for a wall-modeled LES.

Besides the investigation of the numerical scheme, another aim is the validation of the vorticity-based LES filter $\Delta_{\omega}$ in combination with IDDES, according to Eq. (3.20). In Probst and Reuß [G] consistent results are obtained with $\Delta_{\max }$ and $\Delta_{\omega}$ for SAbased IDDES simulations on the three grids at $R e_{\tau}=395$ using the LD scheme. A recent investigation of the influence of the vorticity-based LES filter $\Delta_{\omega}$ is presented in Fig. 4.6. In the SST-based IDDES simulations the Reynolds numbers $R e_{\tau}=1100$ and $R e_{\tau}=4200$ are considered. The velocity profile $u^{+}$and the ratio of the modeled eddy viscosity to the laminar viscosity are compared for $\Delta_{\max }$ and $\Delta_{\omega}$. For both, the LD scheme and the LD2 scheme, the results are consistent, respectively. Only in the outer region of the boundary layer where the LES mode is active the different filter-width definitions have an influence on the eddy viscosity. 


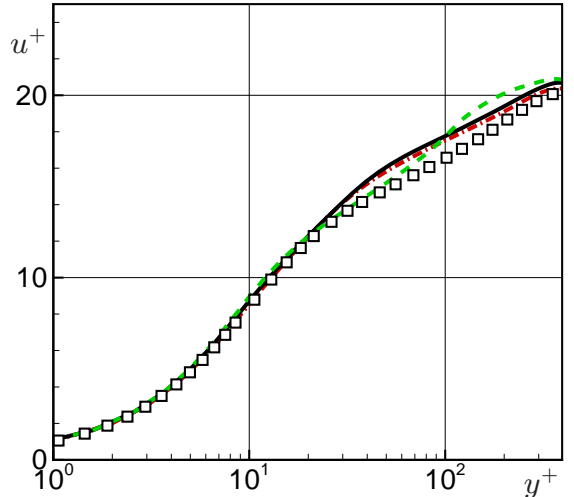

(a) Velocity

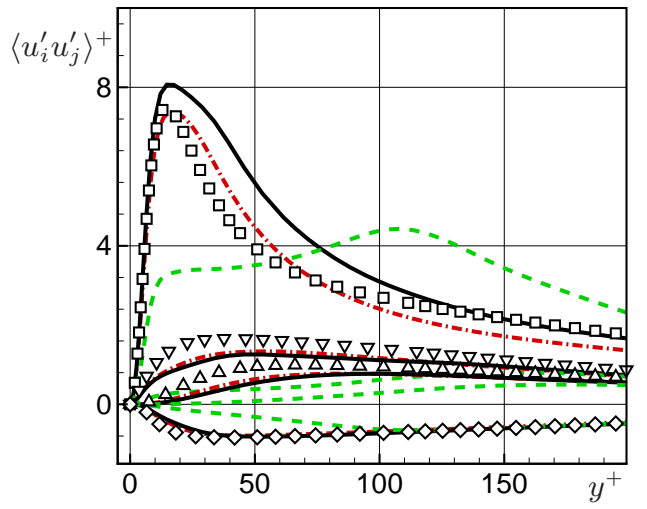

(b) Reynolds stresses

Fig. 4.5: SA-based IDDES grid study of the turbulent channel flow at $R e_{\tau}=395$, reproduced from Probst and Reuß [G]: (-.-.-) $G_{1} ;(-) G_{2} ;(---) G_{3} ;(\square)$ DNS.

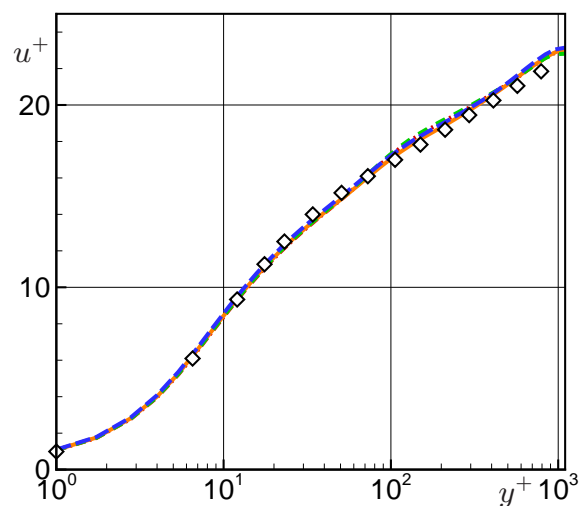

(a) Velocity at $R e_{\tau}=1100$

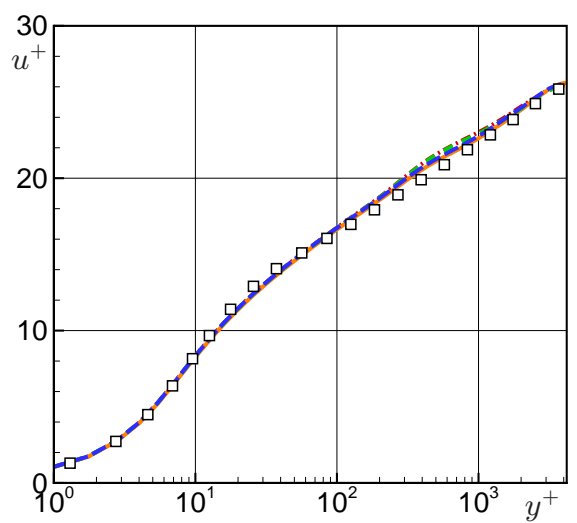

(c) Velocity at $R e_{\tau}=4200$

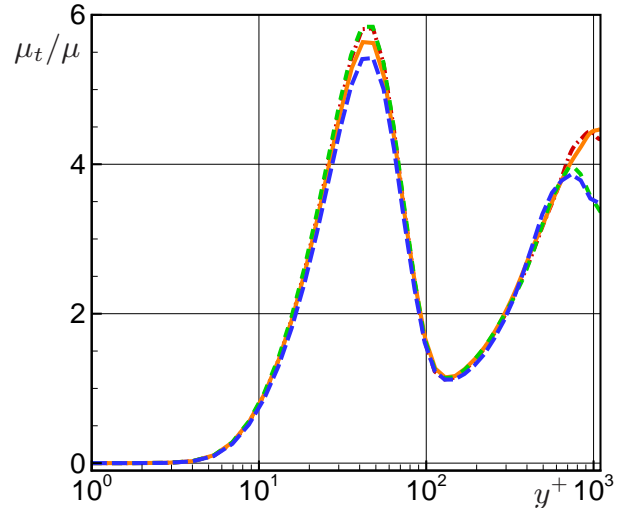

(b) Normalized eddy viscosity at $\operatorname{Re}_{\tau}=1100$

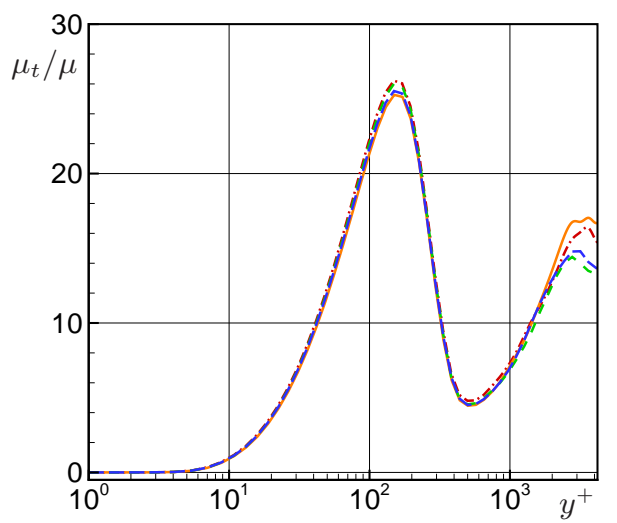

(d) Normalized eddy viscosity at $R e_{\tau}=4200$

Fig. 4.6: Influence of the LES filter width and the numerical scheme on SST-based IDDES simulations of the channel flow at $R e_{\tau}=1100$ and $R e_{\tau}=4200$ : (-.-.-) $\Delta_{\max }$ and LD; (-) $\Delta_{\max }$ and LD2; (- - - ) $\Delta_{\omega}$ and LD; ( - - $\Delta_{\omega}$ and LD2; $(\diamond)$ Reichards law; $(\square)$ DNS. 


\section{Basic assessment of the sponge terms}

In order to test the sponge terms introduced in Sect. 3.3.5, simulations of the turbulent channel flow at $R e_{\tau}=395$ are conducted using non-periodic inflow and outflow boundary conditions and predefined model regions. The simulations are initialized with a steady RANS solution and employ the LD scheme as well as the vorticity-based filter width $\Delta_{\omega}$. Close to the inflow boundary, synthetic turbulence is introduced in the flow, c.f. Sect. 3.3.4.

The computational domain is subdivided into three sections: an inflow region, $x / \delta \in$ $(0,5)$, where the RANS mode is prescribed, a wall-modeled LES region, $x / \delta \in(5,32)$, and another RANS region, $x / \delta \in(32,51.2)$. The synthetic turbulent structures are introduced at the RANS-to-LES interface, marked by the light green area in Fig. $4.7(\mathrm{a})$ at $x / \delta \in(5,5.5)$. The reference values for the turbulence generator are taken from the region $x / \delta \in(4,4.5)$, indicated by the dark green area. The resolved structures are visualized by an isosurface of the $Q$-criterion ${ }^{1}$, colored with the spanwise velocity. The grayscale visualizes the level of the modeled turbulent kinetic energy $k$ from the underlying SST-RANS model. While the synthetic turbulent structures are generated in the forcing region, the level of $k$ decreases automatically.
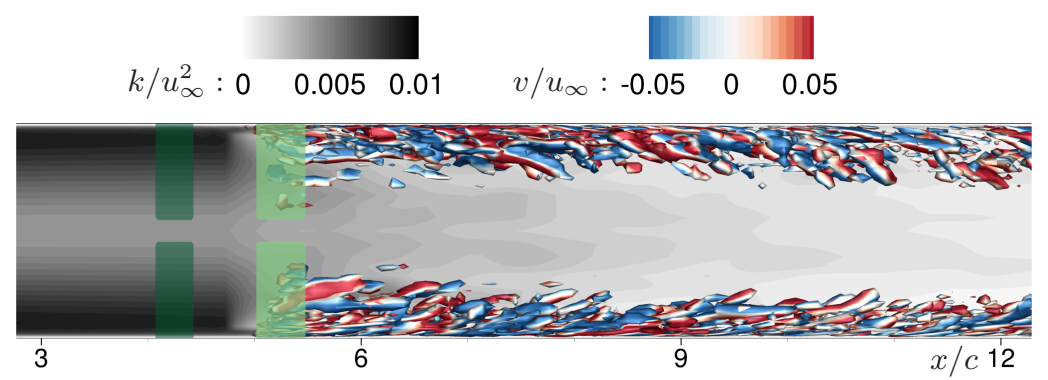

(a) RANS-to-LES interface: generation of synthetic turbulent structures

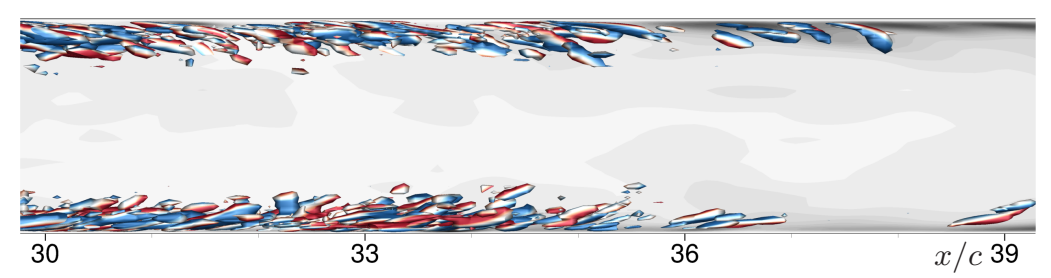

(b) LES-to-RANS interface: no sponge term

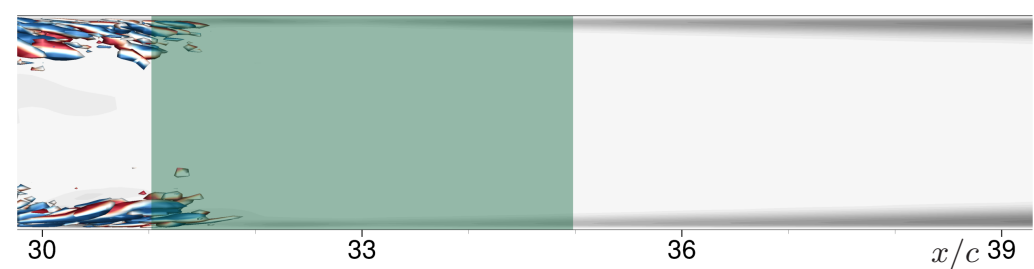

(c) LES-to-RANS interface: sponge term in momentum equation

Fig. 4.7: Generation and damping of resolved turbulent structures in an SST-based ADDES of the turbulent channel flow using synthetic turbulence and sponge terms.

\footnotetext{
${ }^{1}$ The $Q$-criterion is given by $Q=\frac{1}{2}\left(\|\boldsymbol{\Omega}\|^{2}-\|\boldsymbol{S}\|^{2}\right)$, with the vorticity tensor $\boldsymbol{\Omega}$ and the strain rate tensor $\boldsymbol{S}$, according to Hunt et al. [27].
} 
Figs. 4.7(b) and (c) depict the LES-to-RANS interfaces from two different simulations. In (b) no sponge terms are applied and the resolved turbulent structures are preserved over a length of several boundary layer thicknesses in the RANS region. This ambiguous behavior of the turbulence model is as undesirable as the grey area at the RANS-to-LES interface. Therefore, in (c) a sponge term is incorporated in the momentum equation, c.f. Eq. (3.24). The sponge region, $x / \delta \in(31,34)$, is indicated by the green area. With the help of the sponge term the resolved structures dissolve immediately. However, the level of modeled $k$ recovers only slowly in the RANS region. The sponge term in the $k$-equation, according to Eq. (3.25), is intended as a solution, but in the present test applications it was found that the current implementation is not suited: since local mean values are used as target values for the source terms, the sponge is active in the reference region itself. While this is not critical for the mean velocities used in the momentum sponge terms, the velocity correlations are massively distorted. To obtain better reference values for the $k$-sponge term, they should be taken from farther upstream in the future.

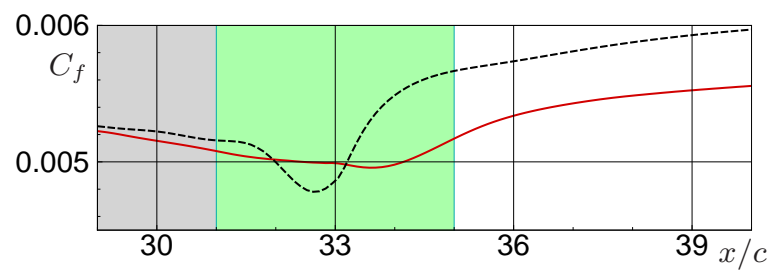

Fig. 4.8: Influence of the sponge terms on the distribution of the skin friction $C_{f}$ along the channel flow computed with SST-ADDES and with synthetic turbulence: $(\mathbf{-}-\mathbf{-})$ no sponge; (—) sponge term in momentum equation.

In Fig. 4.8 the influence of the sponge term on the skin friction $C_{f}$ at the LESto-RANS interface is investigated. The green area indicates where the sponge is active. Without the sponge terms, a strong disturbance in the $C_{f}$ distribution can be observed downstream of the interface, whereas with the activated sponge term the curve shape is considerably smoother.

\subsection{Backward-facing step flow}

The flow over a backward-facing step is a generic test case for a geometry driven separation at a discontinuity in the surface slope. Fig. 4.9 illustrates the streamlines: upstream of the step the turbulent boundary layer is attached. The flow separates from the surface as it passes the step, and a recirculation region forms in the wake. Farther downstream the flow reattaches at the lower wall. The Reynolds number based on the step height $h$ is $R e=37,500$ and the Mach number is $M a=0.13$. Experimental reference data for these flow conditions are available from Driver and Seegmiller [14].

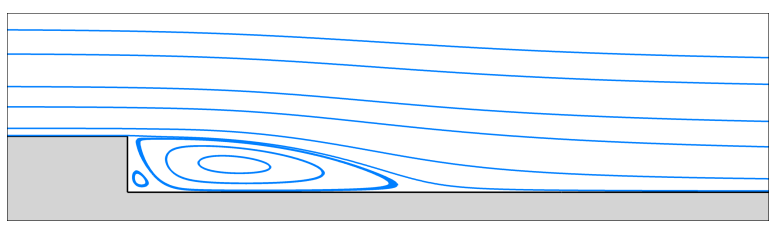

Fig. 4.9: Streamlines for the flow over a backward-facing step. 


\section{Computational setup}

The computational domain and the family of grids that are utilized in the investigations of the backward-facing step are described in Reuß et al. [D] (see Appx.). The characteristic length scale in this test case is the step height $h$. At the inlet, a Dirichlet boundary condition is utilized to prescribe a turbulent boundary-layer profile, which is obtained from experimental data.

The algorithm for the flexible detection of wall-normal lines, described in Sect. 3.3, is generally able to work with intersecting lines. This is the case for the lines that start on the vertical and horizontal walls near the lower corner. However, the assignment of the nearest wall point to the field points, according to Eq. (2.2), is discontinuous over the angle bisector of the corner. This might cause discontinuities in the field distribution of the computed algebraic boundary-layer quantities $\delta_{99}$ and $H_{12}$ and, as a result, disturbances of the flow in the LES region. For this reason, the algebraic sensors are only used to detect the boundary layer on the upper wall, whereas downstream of the step the standard delay functions of the DDES or IDDES are used, respectively.

\section{Results}

In Reuß et al. [D] (see Appx.) the IDDES approach in combination with the filter scale $\Delta_{\omega}$ is applied. Results from precursor investigations, which motivated this choice, are shown in the following.

Fig. 4.10 illustrates the influence of the filter-scale formulation on IDDES simulations of the backward-facing step. The vorticity-based formulation using $\Delta_{\omega}$, according to Eq. (3.20), is compared to the original formulation using $\Delta_{\max }$, according to Eq. (3.19). The resolved Reynolds shear stress $\left\langle u^{\prime} v^{\prime}\right\rangle$ visualizes the generation of resolved turbulent structures.

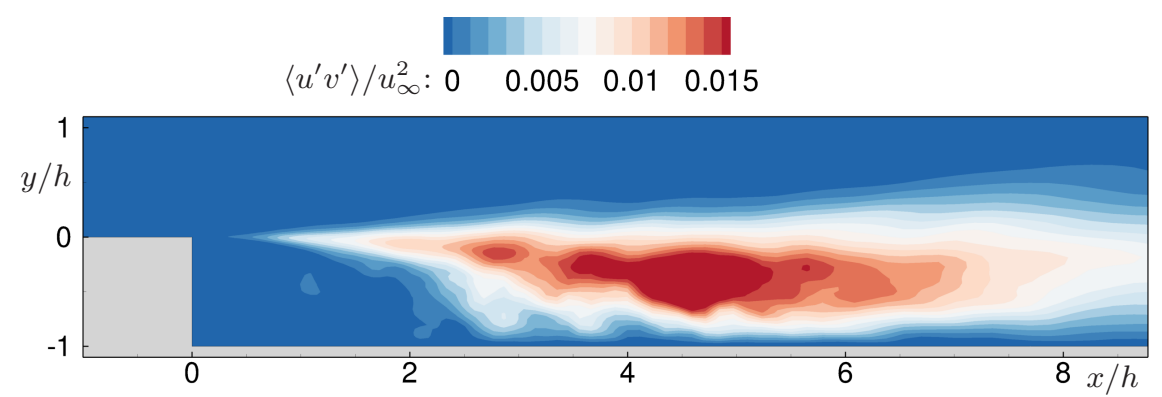

(a) $\Delta_{\omega}$

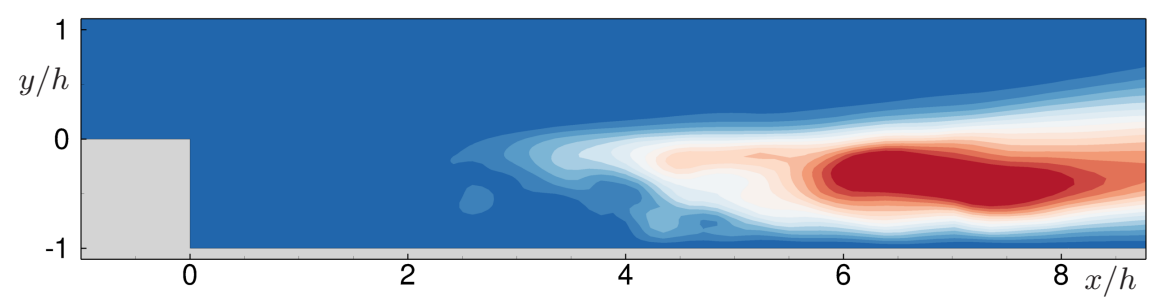

(b) $\Delta_{\max }$

Fig. 4.10: Influence of the LES filter width on the generation of resolved turbulence downstream of the backward-facing step, visualized by the Reynolds shear stress. 
With the vorticity-based LES filter $\Delta_{\omega}$, resolved turbulent structures develop close to the step, see Fig. 4.10(a). With $\Delta_{\max }$ in (b), the generation of resolved turbulent structures is considerably delayed. Therefore, the shear layer is subjected to less turbulent mixing, which has a stabilizing effect on the flow and leads to a delayed reattachment. This is confirmed by the distribution of the skin friction $C_{f}$ in Fig. 4.11. The length of the recirculation region, which is characterized by negative values of $C_{f}$, is overpredicted using $\Delta_{\max }$, while the results of the DDES and the IDDES based on $\Delta_{\omega}$ are both in better agreement with the experimental data in the recirculation region.

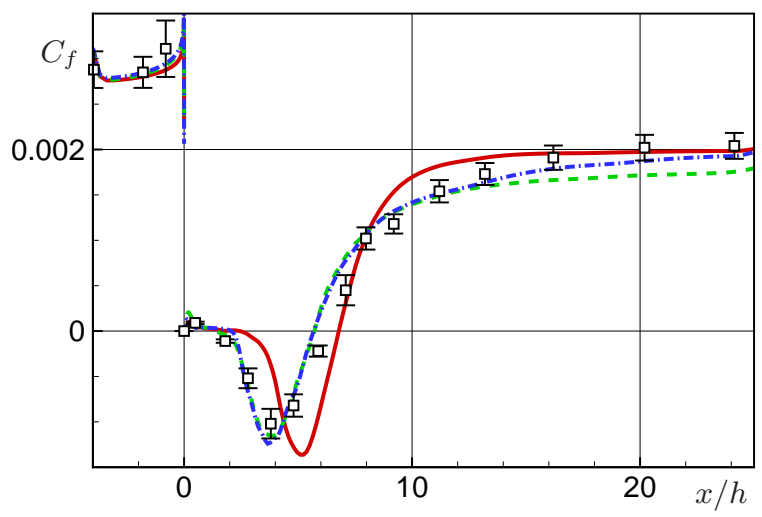

Fig. 4.11: Skin friction distribution for the backward-facing step: (- - SST-DDES with $\Delta_{\max } ;(---)$ SST-DDES with $\Delta_{\omega} ;(-\cdot-\cdot-)$ SST-IDDES with $\Delta_{\omega}$.

However, Fig. 4.11 illustrates another sensitivity of the hybrid RANS/LES modeling approach: the results depend on the treatment of the reattached flow. Resolved structures from the recirculation region are convected downstream of the reattachment point. With the DDES approach the reattached boundary layer is modeled with RANS, as the $f_{d}$ function detects the boundary-layer edge without considering the resolved turbulence. The resolved structures interfere with the RANS model and deteriorate the solution. Opposed to that, in the IDDES approach the resolved structures activate the wall-modeled LES mode in the reattached boundary layer. Therefore, the IDDES result shows the best agreement with the experimental data in Fig. 4.11.

In Reuß et al. [D] (see Appx.) the grid-resolution sensors $S$ from Sect. 3.2.2 are evaluated for a family of grids for the flow over the backward-facing step. The sensor values are used as input for the DLR-TAU grid-adaptation tool to perform a local grid refinement. In Fig. 4.12, results computed on these locally refined grids are compared to reference results from globally refined grids. The locally refined regions in the grids $G 2 A$ and $G 3 A$ are outlined in black. In the refined region the grid $G 2 A$ provides the same resolution as the globally refined grid $G 1$. Likewise, the grids $G 3 A$ and $G 2$ provide the same resolution in the outlined region. The color contour illustrates the distribution of the sensor values, which are recomputed on the locally refined grids. For the pair of grids $G 1$ and $G 2 A$ similar values are obtained, indicating that the sensor correctly detects a similar LES resolution capability. The same holds for the grids $G 3 A$ and $G 2$. As sensor values $S<0.8$ indicate underresolved LES regions (c.f. Sect. 3.2.2), it can be concluded that only the finest grids $G 1$ and $G 2 A$ provide sufficient resolution for a reliable LES. The comparison shows, that the resolution sensor successfully detects the underresolved region in the grid $G 2$, and that the local refinement resulting in grid $G 2 A$ enhances the reliability of the solution. 
G1 - SST IDDES $\triangle$

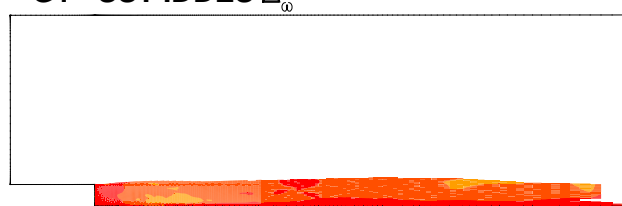

G2 - SST IDDES $\triangle$

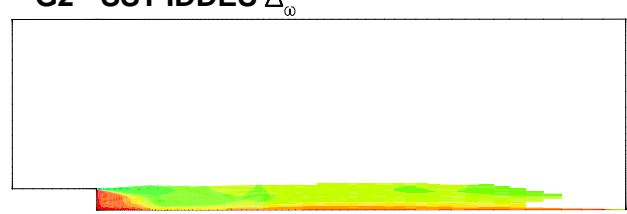

G3 - SST IDDES $\triangle$

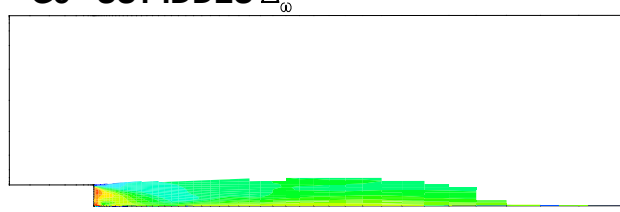

\section{G2A - SST IDDES}

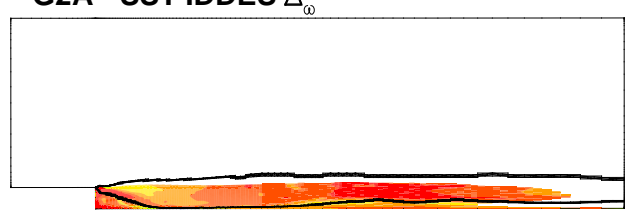

G3A - SST IDDES

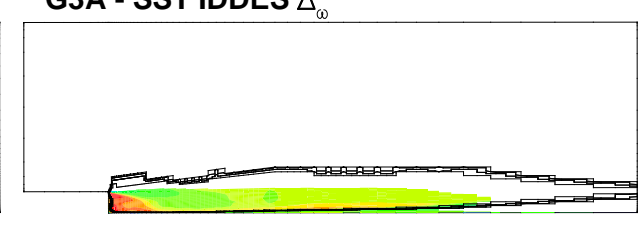

$\mathrm{S}=\mathrm{k}_{\mathrm{res}} / \mathrm{k}_{\text {tot }}: \quad \begin{array}{llll}0.6 & 0.7 & 0.8 & 0.9\end{array}$

Fig. 4.12: Comparison of the grid sensor values on a family of grids. The values are blanked if the resolved turbulence intensity is smaller than $T_{0}=0.1$. The globally refined grids are shown on the left, the locally adapted grids are shown on the right.

\subsection{Wall-mounted hump flow}

The test case of the flow over a wall-mounted hump was designed with the focus on turbulence model validation, see Greenblatt et al. [23]. The first challenge for turbulence models is the accurate prediction of the location of the pressure-induced separation from the smooth surface. The second challenge is the correct prediction of the reattachment point. Experimental data are available from Naughton et al. [53].

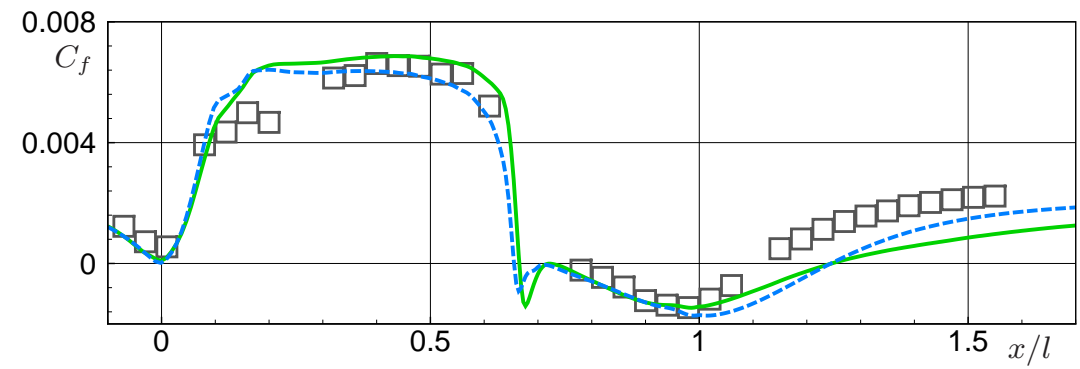

Fig. 4.13: Comparison of skin friction distribution on the wall-mounted hump: ( - ) SA-RANS; (- - -) SST-RANS; ( $\square$ ) measurements.

Fig. 4.13 shows TAU results of the skin friction distribution $C_{f}$ for SA-RANS and SST-RANS. Both RANS models overestimate the length of the recirculation region, characterized by negative values of $C_{f}$, and predict the reattachment point too far downstream. 


\section{Computational setup}

The geometry is described in terms of the hump length $l$. A section of the $\operatorname{grid}^{2}$ in the region of the hump is shown in Fig. 4.14. In the stream-wise direction the computational domain extends from $x / l=-2.14$ to $x / l=4$ and is resolved with 511 points. The height of the domain is $0.912 l$, resolved with 127 points, and the first point above the wall is located at $y^{+}=1$. In the span-wise direction the computational domain extends over $0.4 l$ and is discretized with 81 points, which yields mostly isotropic cells in the separation region.

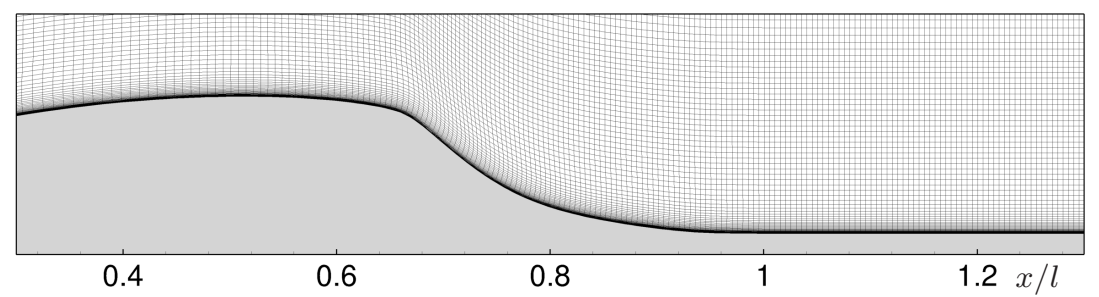

Fig. 4.14: Computational grid for the wall-mounted Hump.

The upper wall is considered as inviscid, i.e. only the velocity component perpendicular to the wall is suppressed. Above the hump, the upper wall is drawn downwards in order to take into account the blockage effects in the experiments. At the inlet, a Dirichlet boundary condition is applied to prescribe a turbulent inflow profile. The Reynolds number based on the hump length $l$ is $R e=936,000$ and the Mach number is $M a=0.1$.

\section{Results}

In Sect. 3.3.2 the algebraic sensors of the ADDES were introduced by reference to the flow over the wall-mounted hump. Fig. 4.15 depicts the ADDES shielding function $\tilde{f}_{d, \mathrm{ADDES}}$, which results from the automatic modifications by the algebraic sensors. The blue region is computed in RANS mode, while in the red region the wall-modeled LES mode is active. The dashed line indicates the shape of the original IDDES shielding function $\tilde{f}_{d}$ for reference.

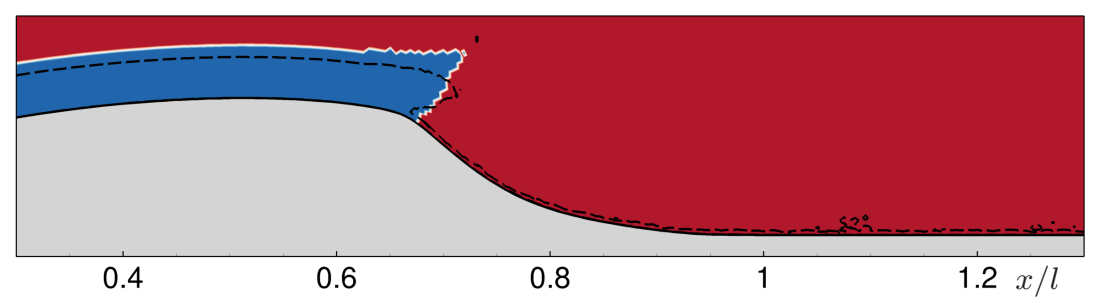

Fig. 4.15: Shielding function $\tilde{f}_{d, \mathrm{ADDES}}$, modified through the ADDES sensors, for the wallmounted hump.

Fig. 4.16 compares the skin friction coefficient $C_{f}$ from the ADDES approach with a reference SST-RANS result. In the ADDES, the LES filter width $\Delta_{\omega}$ is applied. While in the ADDES the predicted length of the recirculation region is in better

\footnotetext{
${ }^{2}$ The grid and the settings for this test case are taken from the EU project Go4Hybrid, c.f. Mockett et al. [50].
} 
agreement with the experiment than in the RANS approach, the result is still not satisfactory.

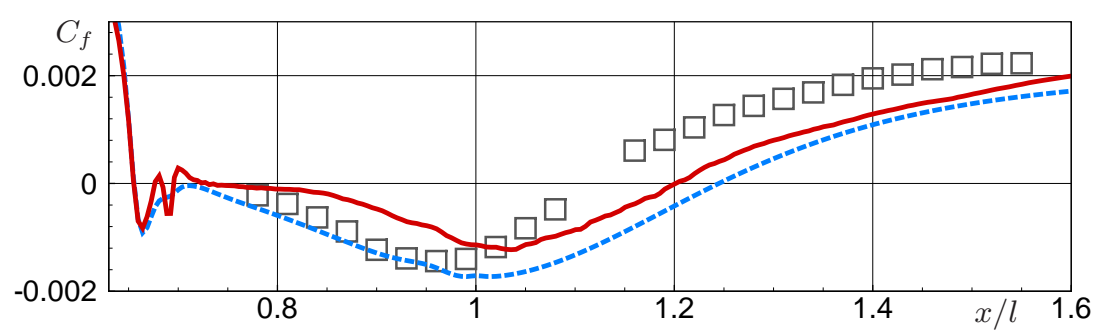

Fig. 4.16: Skin friction distribution on the wall-mounted hump: ( - - -) SST-RANS; $(-)$ SST-IDDES with $\Delta_{\omega} ;(\square)$ reference from experiments.

Fig. 4.17 presents the ratio of $\Delta_{\omega}$ to $\Delta_{\max }$ in the recirculation region downstream of the wall-mounted hump. This comparison illustrates a limitation of the vorticitybased LES filter $\Delta_{\omega}$ : while for high-aspect-ratio cells the values of $\Delta_{\omega}$ are considerably smaller than the more conservative $\Delta_{\max }$, this is not the case for nearly isotropic cells. On the contrary, for isotropic cells $\Delta_{\omega}$ may yield even slightly larger values than $\Delta_{\max }$. In the present grid the recirculation region is resolved with nearly isotropic cells, and in Fig. 4.17 the ratio is close to one in this region. Therefore, it can be stated that $\Delta_{\omega}$ mainly suppresses the unfavorable effect of anisotropic grid cells in LES regions, e.g. close to the surface discontinuity of the backward-facing step in Sect. 4.3. However, in LES regions with isotropic cells, only little positive effects can be expected from the vorticity-based filter $\Delta_{\omega}$. Precursor simulations, which are not shown here, indicate that it is necessary to add synthetic turbulence in order to obtain further improvements in this case.

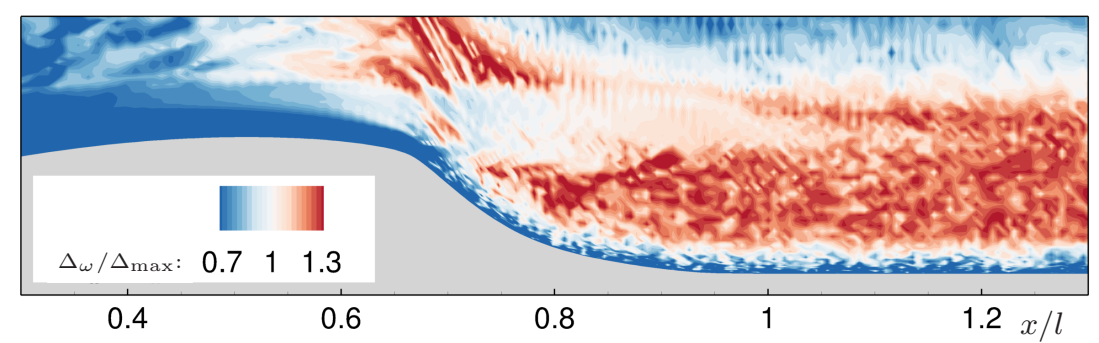

Fig. 4.17: Ratio of $\Delta_{\omega}$ to $\Delta_{\max }$ in the recirculation region of the wall-mounted hump.

\subsection{Two-element airfoil with undisturbed onflow}

This test case is considered as the first preparation step towards the target application, where a lateral vortex interacts with a high-lift airfoil. The DLR F15 two-element airfoil corresponds to the geometry of the three-element configuration in Sect. 4.1, but with a retracted slat and a modified flap position. Variations of the flap position were investigated by Scholz et al. [69]. In the experiments the model airfoil is equipped with droop noses at the junction with the wind tunnel, which reduce the influence of the side-wall boundary layers on the flow over the airfoil. With these devices, the flow in the centerline section of the wind tunnel remains essentially undisturbed, see Hahn et al. [24]. 


\section{Computational setup}

Details about the grids that are used in the preparatory RANS simulations can be found in Reuß et al. [E] (see Appx.). In the RANS simulations two incidence angles of the high-lift airfoil are considered: at $\alpha=0^{\circ}$ the flow is separated on the flap, while it is attached at $\alpha=6^{\circ}$.

In the hybrid RANS/LES simulations, which are presented here for the first time, the incidence angle of $\alpha=0^{\circ}$ is considered. The vortex-generator airfoil from the target application is included in the computational setup. The purpose is to use the results from the precursor investigation as initial solution for the target application. The span-wise grid adaptation, described in Sect. 3.2.1, is applied to provide a high local resolution for the hybrid RANS/LES approach in the region around the flap. With this approach, the number of points in the grid is reduced to $26.6 \times 10^{6}$ compared to $47.4 \times 10^{6}$ points in a globally refined grid.

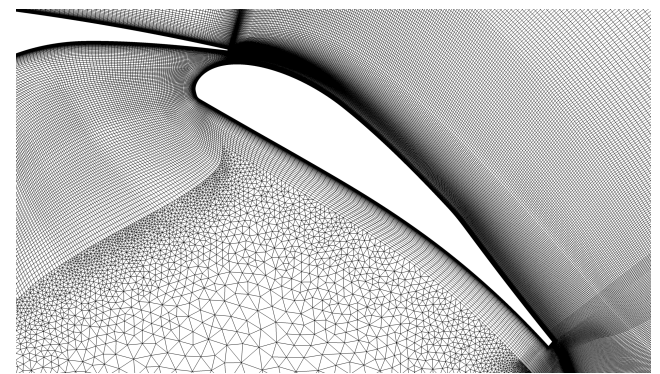

Fig. 4.18: Structured region of the grid near the flap of the DLR F15 two-element airfoil.

Fig. 4.18 presents the structured region of the grid for the two-element airfoil. In contrast to the grid for the three-element airfoil, see Fig. 4.3, a H-type grid topology is used at the trailing edges, and the structured layers cover the whole recirculation region above the flap. The flow is determined by a Reynolds number of $R e=1.9 \times 10^{6}$ based on the retracted chord length $c$ and a Mach number of $M a=0.15$.

\section{Results}

In Reuß et al. [E] (see Appx.) a suitable simulation strategy is presented, which gives results that are comparable to the experiments. RANS simulations are used to investigate if the whole wind-tunnel setup must be considered, including the side walls and the drooped noses of the airfoil model. To this end, two approaches are compared: in the first approach the whole setup is included in the three-dimensional grid, while in the second approach the grid is restricted to the center-line section of the wind tunnel. The solutions in the center-line section agree well for the two approaches, which confirms that the computational domain can be restricted to the center-line section of the wind tunnel.

Moreover, Reuß et al. [E] (see Appx.) compare RANS simulations using the SST model with simulations using the JHh-v2 Reynolds stress model. It is found that the RSM does not yield a stable solution; instead the flow exhibits periodic vortex shedding from the cove region, which was not observed in the experiments. To eliminate this uncertainty from the investigations, all hybrid RANS/LES applications in the present thesis are based on the SST model. 
In the following, results from three SST-based hybrid RANS/LES simulations are presented. In the reference simulation, $\mathrm{HRLM}_{r e f}$, the standard numerical settings of TAU, the LES filter $\Delta_{\max }$, and the original IDDES delay function $f_{d t}$, according to Eq. (1.53), are used. In the optimized simulation, $\mathrm{HRLM}_{\text {opt }}$, the low-dissipation (LD) scheme and the LES filter $\Delta_{\omega}$ are applied. Moreover, the delay function is modified by the algebraic sensors, which detect the edge of the boundary layer with the $\delta_{99}$-criterion according to Eq. (3.11). In the $\mathrm{HRLM}_{o p t+S T}$ the same settings as in HRLM $_{\text {opt }}$ are used, but in addition synthetic turbulence is fed into the flow, see Sect. 3.3.4.

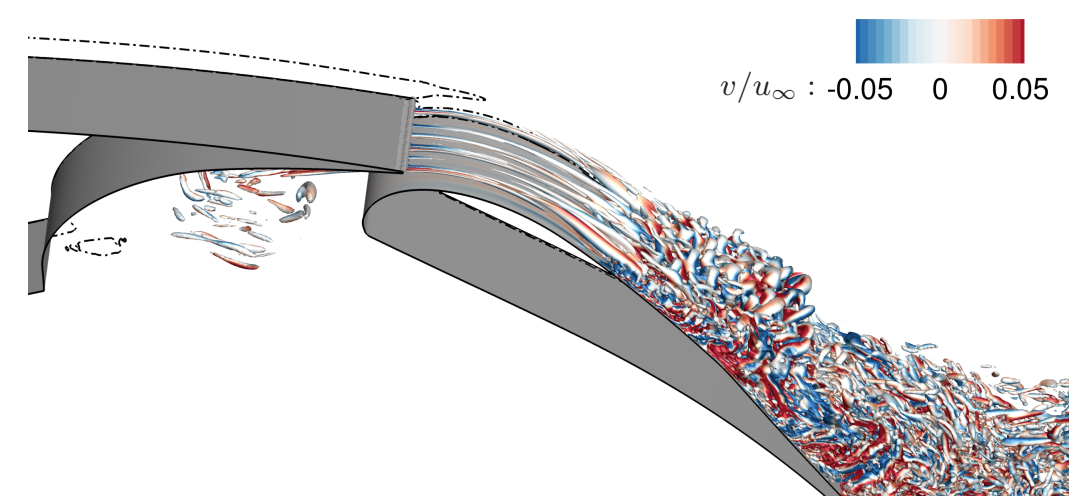

(a) Reference model/numerics

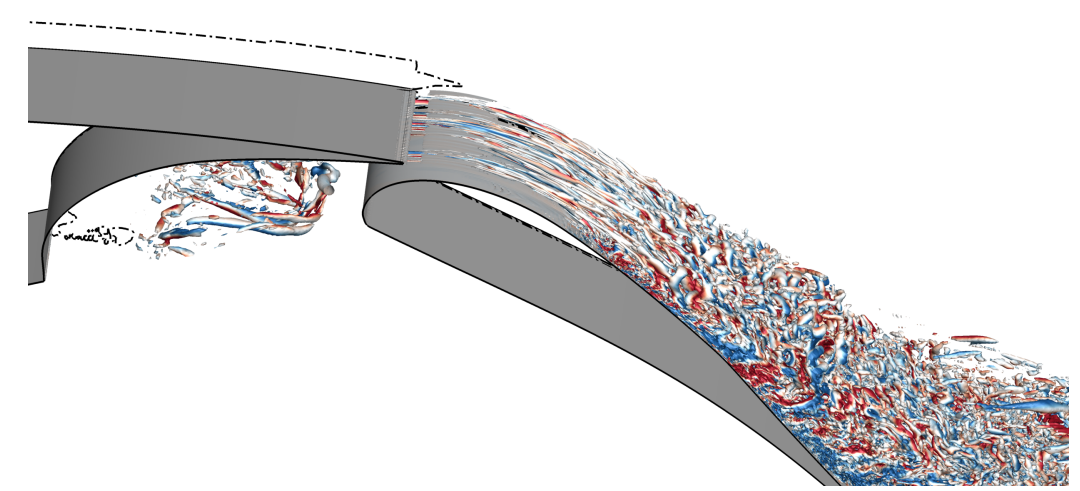

(b) Optimized model/numerics

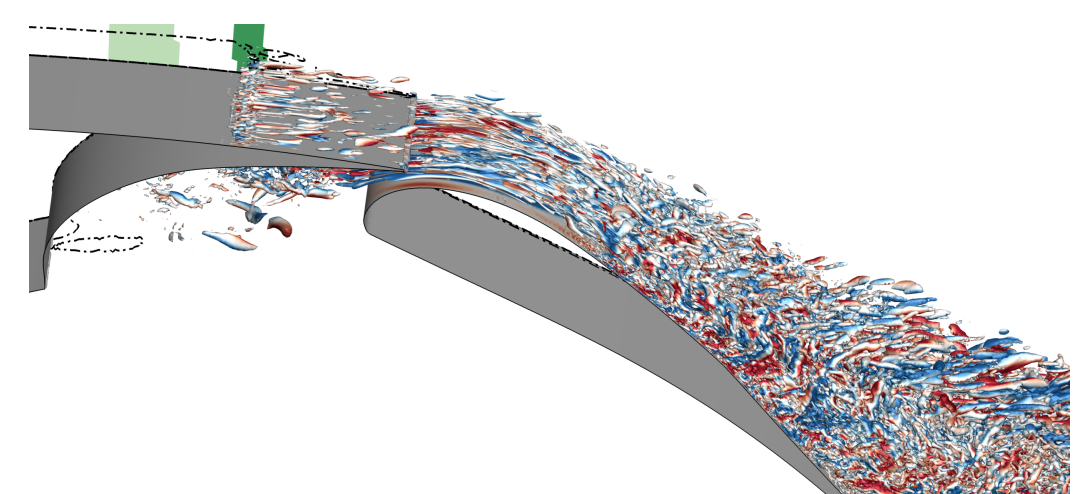

(c) Optimized model/numerics and synthetic turbulence

Fig. 4.19: Resolved turbulent structures above the flap of the two-element airfoil visualized by an isosurface of the $Q$-criterion, colored by the span-wise velocity. 
In Fig. 4.19 the $Q$-criterion visualizes the turbulent structures in the region above the flap. The dashed lines denote the interfaces between RANS and LES, detected by the switching function. The forcing region in the $\mathrm{HRLM}_{o p t+S T}$ simulation is located at an user-defined position of $x / c=0.83$ near the main-wing trailing edge, and the separation sensor is modified to switch to wall-modeled LES at this location. The synthetic turbulent structures are excited over a length of one boundary layer thickness, while the reference values for the synthetic turbulence generator are taken from farther upstream. In Fig. 4.19(c) the reference region and the forcing region are indicated by the dark green and light green areas, respectively.

In comparison to the reference simulation HRLM $_{r e f}$ in Fig. 4.19(a), much finer structures are resolved in both simulations that use the optimized settings, i.e. the $\mathrm{HRLM}_{\text {opt }}$ in (b) and the HRLM $\mathrm{H}_{\text {opt }+S T}$ in (c). In (a) and (b) the wake downstream of the main-element trailing edge is quite stable, and the generation of the resolved turbulent structures is delayed. This shows that the effect of $\Delta_{\omega}$ is not sufficient to trigger the breakup of the shear layer. In contrast, the resolved turbulent structures introduced by the synthetic forcing lead to an early breakup of the shear layer in (c).

In Fig. 4.20 the normalized mean-velocity vectors of the HRLM $\mathrm{H}_{\text {opt }}$ are compared with PIV data from Klein et al. [32]. The color contour indicates the magnitude of the mean velocity. The general flow topology and the velocity distribution are predicted in good agreement with the experiment.

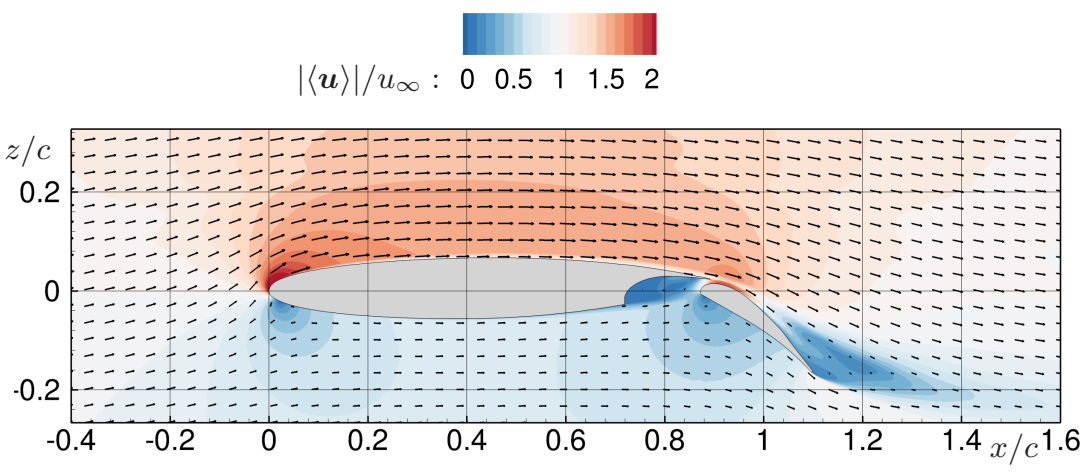

(a) HRLM $_{o p t}$

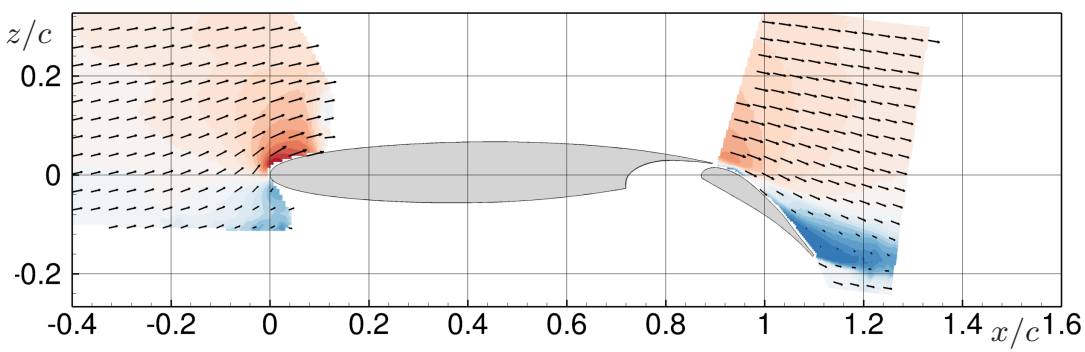

(b) PIV

Fig. 4.20: Mean-velocity vectors in the flow about the two-element high-lift airfoil at undisturbed inflow.

Fig. 4.21 shows details of the flow about the flap using the stream-wise velocity component $u$. In the depicted region the wake of the main wing, the flow through the gap, and the boundary layer on the flap conflate leading to a complex mutual 
interaction. An apparent difference between the experiment and the simulations is the flow separation on the flap. In the PIV data in (a), the separation is clearly illustrated by the streamlines. While the two-dimensional reference RANS simulation in (b) reproduces the separation in general, the size of the recirculation region is overpredicted. Opposed to that, in all hybrid RANS/LES simulations the flow on the flap is fully attached, which is exemplarily shown for the $\mathrm{HRLM}_{o p t+S T}$ in (c).

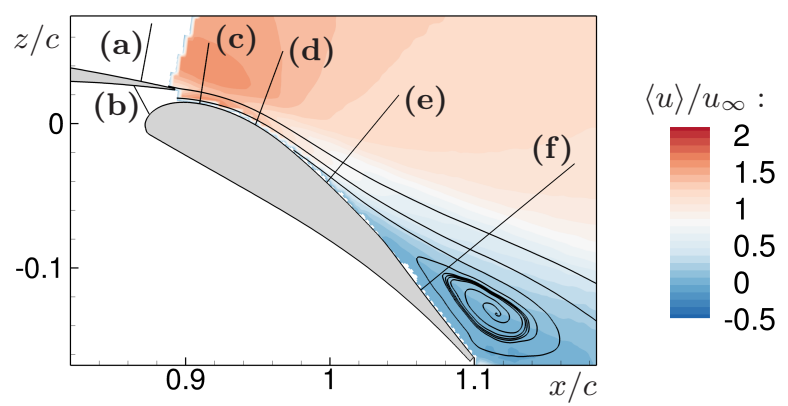

(a) PIV

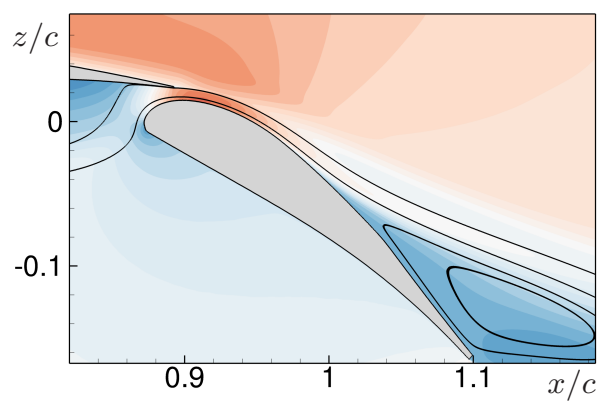

(b) RANS

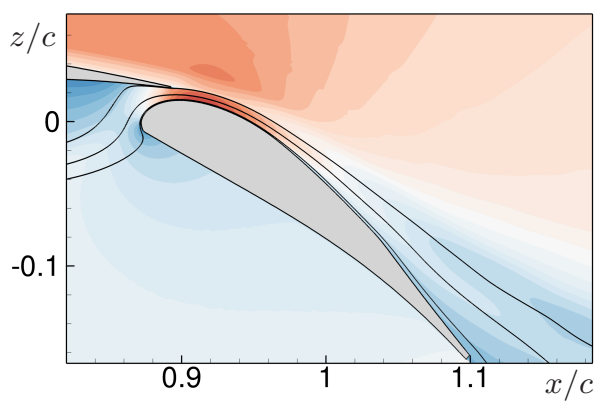

(c) HRLM $_{o p t+S T}$

Fig. 4.21: Mean stream-wise velocity component for the flow about the flap of the twoelement high-lift airfoil at undisturbed inflow.

To study the confluent shear layers in detail, Fig. 4.22 depicts the velocity profiles along the lines indicated in Fig. 4.21(a). For the boundary layer near the trailing edge of the main wing, shown in 4.22(a), all simulations predict the attached flow in good agreement, but no experimental reference data are available. Profile (b) compares the flow through the gap between the main wing and the flap, again without reference PIV data. The position $\Delta z / c=0$ is located on the main wing and $\Delta z / c=-0.012$ is located on the flap. With the RANS approach the velocity magnitude in the center of the gap is smaller, and this difference is maintained in the following profiles (c) and (d). While the PIV data near the wall are afflicted with larger uncertainties, at least the trend towards a lower gap-flow velocity is considered correct, as it additionally increases the separation tendency of the flow.

In the profiles (c) and (d) the main-wing wake appears as sharp kink separating the boundary layer on the upper side of the main wing from the gap flow. In profile (c) the kink is much more pronounced in all simulations than in the PIV data. In profile (d) the severity of the kink is reduced by turbulent mixing: while the turbulence model provides high values of eddy viscosity in the RANS approach, in the hybrid RANS/LES simulations the resolved structures are mainly responsible for the mixing, as the sub-grid model produces little modeled turbulence in LES mode. Therefore, in 


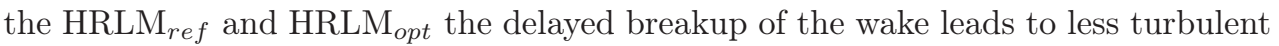
mixing, whereas the resolved turbulent structures which are excited by the synthetic forcing in the HRLM $\mathrm{H}_{o p t+S T}$, smoothen the kink slightly.

Despite the delayed breakup of the main-wing wake, in $\mathrm{HRLM}_{\text {opt }}$ the effect of the resolved turbulence has increased to a level comparable to the $\mathrm{HRLM}_{o p t+S T}$ and the shape of profile (e) is similar for both simulations. The main differences between the simulations must be contributed to the different separation behavior which leads to different paths of the main-wing wake. In profile (f) the wake is essentially smoothened out in the PIV data, while it is preserved in all simulations, including the RANS simulation, where the flow is separated.

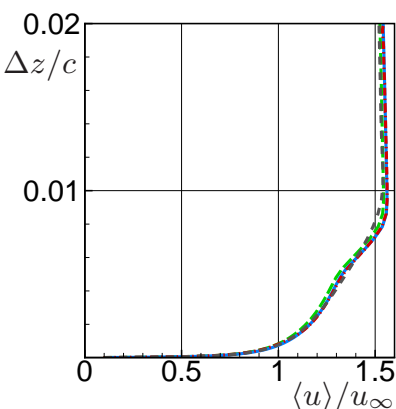

(a)

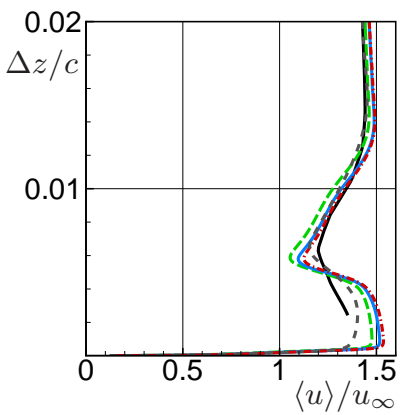

(d)

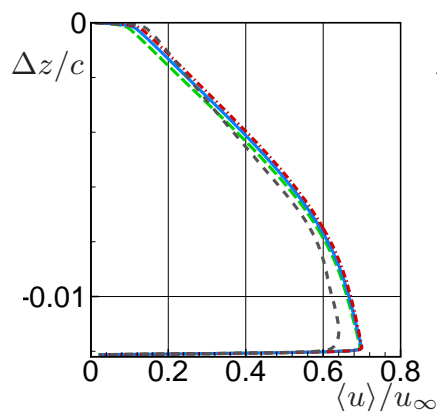

(b)

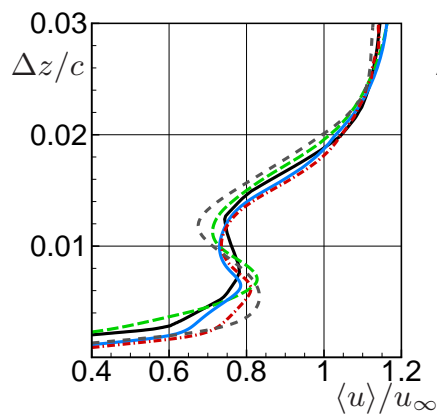

(e)

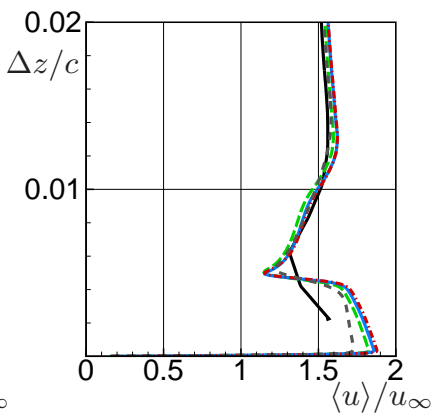

(c)

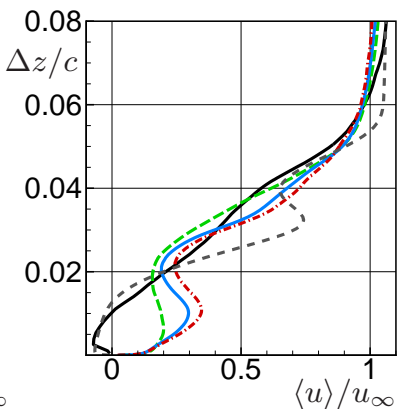

(f)

Fig. 4.22: Velocity profiles in several positions near the flap of the two-element airfoil (indicated in Fig. 4.21(a)): (- - HRLM $_{r e f}$; (- HRLM $_{\text {opt }} ;(-\cdot-\cdot-)$ HRLM $_{o p t+S T} ;(-\mathbf{-})$ two-dimensional RANS;

) PIV data.

Fig. 4.23 illustrates one uncertainty in the simulations: the influence of the transition modeling. In the experiment transition was tripped at $x / c_{\text {flap }}=0.31$, which is adopted to prescribe transition in the simulations ${ }^{3}$. Fig. 4.23(a) depicts the skin friction $C_{f}$ on the flap, where negative values indicate flow separation. In (b) the eddy viscosity illustrates the influence of the transition prescription on the flow, where the green line indicates the transition location. In the hybrid RANS/LES approaches, represented by the $\mathrm{HRLM}_{o p t+S T}$, the level of the eddy viscosity is low, and a laminar boundary-layer profile is obtained in the region specified as laminar. In contrast, a high level of eddy viscosity is transported from the turbulent flow in the main-wing cove into the outer boundary layer in the laminar region in the RANS simulation. As a consequence the boundary-layer profile exhibits a premature

\footnotetext{
${ }^{3}$ This leads to the kink in the $C_{f}$ curve.
} 
tendency towards a turbulent shape, which manifests in increased values of $C_{f}$ in the laminar region. Downstream of the transition location, the production term of the turbulence model reacts stronger to the laminar flow in the hybrid RANS/LES approaches, and the eddy viscosity grows more rapidly than in the RANS simulation, leading to higher values of $C_{f}$.

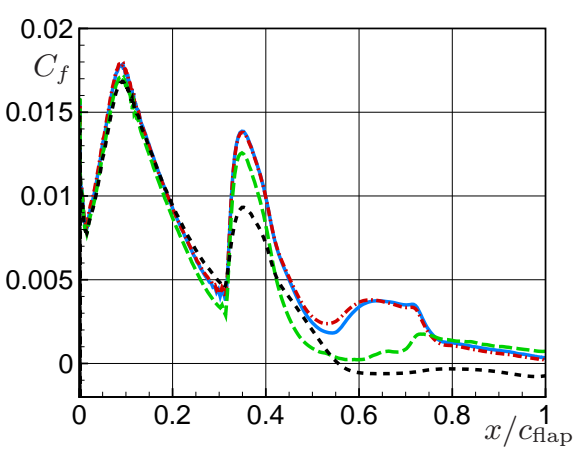

(a) $C_{f}$

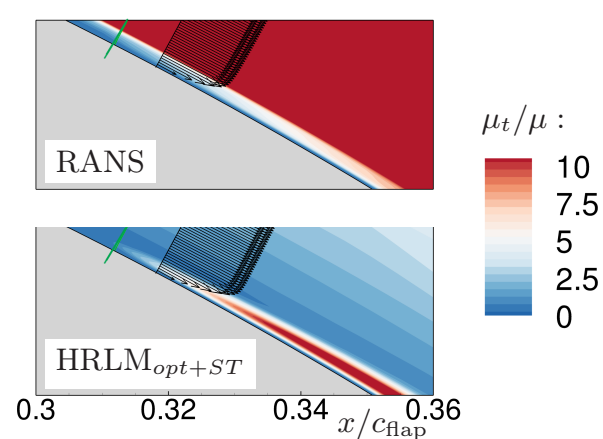

(b) Eddy viscosity

Fig. 4.23: Distribution of the skin-friction $C_{f}$ along the upper side of the flap in (a) (see Fig. 4.22 for the legend); distribution of the eddy viscosity along the flap in (b).

The general question arises, how laminar regions should be treated in scale-resolving simulations, especially in the case where the laminar region is subjected to massive outer turbulence. This source of uncertainty in the simulation could not be resolved in the scope of the present thesis. The limited computational resources did not allow to study the influence of the transition prescription on hybrid RANS/LES simulations. The same problem arises in Reuß et al. [B] (see Appx.) for the flow over the three-element airfoil.

To find an explanation for the pronounced of $C_{f}$-maxima at the rear end of the flap around $x / c_{\text {flap }} \approx 0.55$ in the $\mathrm{HRLM}_{\text {opt }}$ and $\mathrm{HRLM}_{\text {opt }+S T}$ simulations, the vorticity is shown in Fig. 4.24 to illustrate the wake of the main wing. At $x / c_{\text {flap }} \approx 0.55$ the main-wing wake merges with the boundary layer on the flap and which locally increases the turbulent momentum transport. It seems, that mainly the small resolved structures in the HRLM $\mathrm{Hpt}_{\text {ond }}$ and $\mathrm{HRLM}_{\text {opt }+S T}$ contribute to the increased level of $C_{f}$.

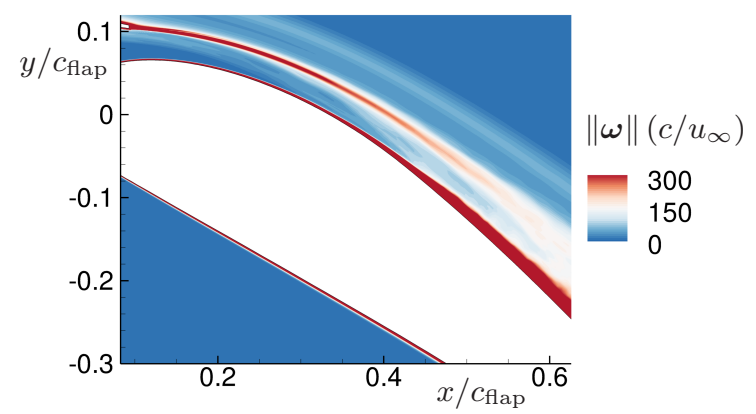

Fig. 4.24: Vorticity above the flap of the high-lift airfoil in the HRLM opt $_{\text {simulation. }}$

Fig. 4.25 depicts the pressure coefficient $C_{p}$ on both elements. In all simulations the major part of the flow about the main element is computed in RANS mode. 
Therefore, on the main wing in (a) $C_{p}$ essentially reflects the RANS solution, and the difference between the three hybrid RANS/LES solutions, the two-dimensional reference RANS, and the experiment is negligible.

According to Fig. 4.25(b), there is a wider spread of the pressure distributions on the flap. Near the suction peak, the HRLM ref $_{\text {agrees with the RANS, while the HRLM }}$ opt and the HRLM $\mathrm{H}_{o p t+S T}$ agree better with the experimental data. This shows the influence of the grey area in $\mathrm{HRLM}_{r e f}$, which is reduced through the improved length scale $\Delta_{\omega}$ in the other scale-resolving simulations. However, in the rear section of the flap, starting at $x / c \approx 0.6$, the results of the three hybrid RANS/LES simulations nearly coincide. The grid cells in this region are almost isotropic, and it was already observed for the flow over the wall-mounted hump, that the effect of $\Delta_{\omega}$ vanishes for isotropic cells. Furthermore, the smaller turbulent structures that are resolved with the low-dissipation scheme in Fig. 4.19, show little effect on $C_{p}$.

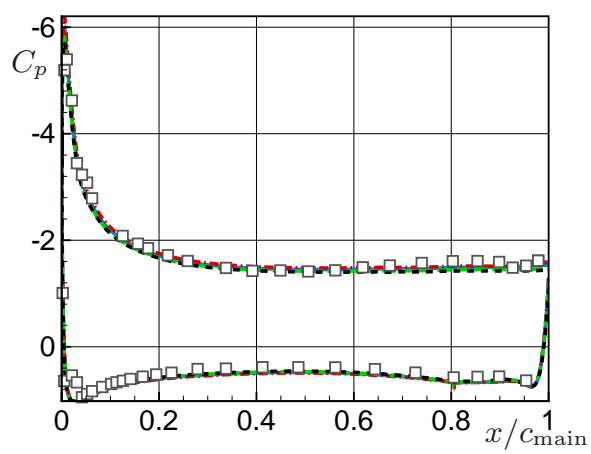

(a) $C_{p}$ on the main wing

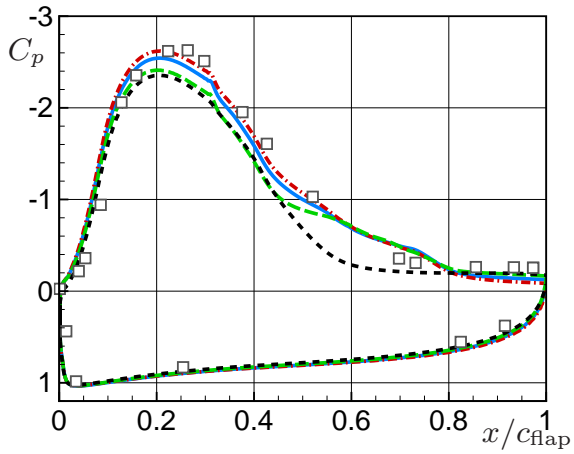

(b) $C_{p}$ on the flap

Fig. 4.25: Pressure distribution $C_{p}$ on the two-element airfoil at undisturbed onflow conditions: (- - $\operatorname{HRLM}_{r e f} ;(\longrightarrow) \operatorname{HRLM}_{o p t} ;(-\cdot-\cdot-) \operatorname{HRLM}_{o p t+S T} ;(-\mathbf{-})$ two-dimensional RANS; $(\square)$ reference from experiment.

For an approximate assessment of the grid resolution, Fig. 4.26 depicts the distribution of the LES grid-resolution sensor $S$, based on Eq. (3.7). The sensor can only provide meaningful results if a certain amount of turbulence is resolved. Therefore, the evaluation is restricted to regions where the resolved turbulence intensity $T_{u}=1 / u_{\text {ref }} \cdot \sqrt{2 / 3\left(\left\langle u^{\prime 2}\right\rangle+\left\langle v^{\prime 2}\right\rangle+\left\langle w^{\prime 2}\right\rangle\right)}$ exceeds a given threshold of $T_{0}=0.1$, the same value as in Reuß et al. [D] (see Appx.).

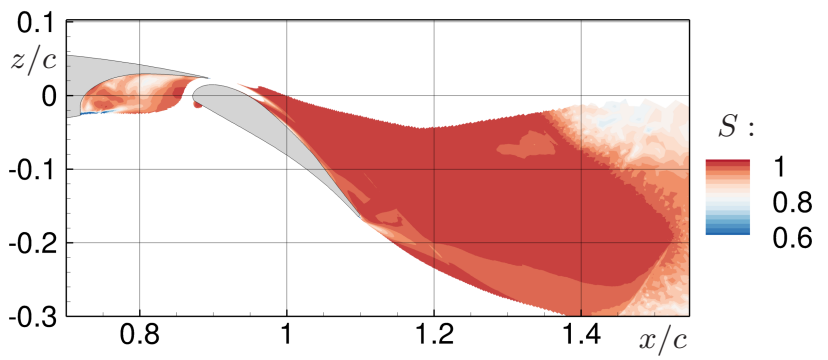

Fig. 4.26: Distribution of the grid-resolution sensor $S$ in the HRLM $_{o p t}$ simulation of the two-element high-lift airfoil. 
In the entire region above the flap, the values of the sensor are larger than $S=0.8$, which confirms sufficient grid resolution for LES. The sensor values drop abruptly in the region $x / c=1.4-1.5$, at the interface between the structured grid layers and the unstructured grid region where the growth rate of the grid cells suddenly increases. Only in a small region of the shear layer in the wing cove, the sensor indicates underresolved flow with values smaller than $S=0.8$. However, this is considered to be a consequence of the grey area, which should be addressed by introducing synthetic turbulence upstream of the edge, rather than refining the grid.

\subsection{Transport of a lateral vortex}

In the target application of the present thesis, an airfoil-generated vortex interacts with a DLR F15 two-element high-lift airfoil. To prepare this simulation, Reuß et al. $[\mathrm{F}]$ (see Appx.) present a suitable numerical representation of the vortex transport. The results are briefly summarized here. Since in the target application the high-lift airfoil is positioned at a distance of 3.33 chord lengths downstream of the vortex generator, the outcome of this investigation has a major influence on the computational effort required for the target application. Experimental reference data are provided by Klein et al. [32].

\section{Computational setup}

In the computational setup the isolated NACA-0021 vortex-generator airfoil is positioned in the wind tunnel. The deflection of the airfoil is achieved with the help of the grid-deformation approach, described in Sect. 2.5 where Figs. 2.5(c) and (d) display the grid before and after the deflection. Two different simulation approaches are compared: on the one hand, the vortex transport is modeled in the LES mode, while on the other hand, a RANS model is used in the transport region. Details about the grid and the simulation strategy are presented in Reuß et al. $[\mathrm{F}]$ (see Appx.).

\section{Results}

Fig. 4.27 presents the resolved turbulent structures in the wake of the deflected vortex-generator airfoil. The bend in the wake illustrates the position of the lateral gust, generated during the airfoil deflection and convected with the mean flow. This represents the vortex, which is considered in the target application. The structures are visualized by an isosurface of the $Q$-criterion, and the color contour displays the span-wise velocity.

Two hybrid RANS/LES simulations are compared, which both apply the vorticitybased LES filter $\Delta_{\omega}$ and the low-dissipation settings. In (a) the quasi two-dimensional rollers in the wake are very stable, and no resolved turbulent structures develop. Therefore, in (b) the generation of turbulent structures is triggered by local stochastic forcing of the flow near the trailing edge.

To investigate the effect of the passing vortex, the induced angle of attack $\alpha_{\text {ind }}$ and the disturbance velocity $u_{\text {ind }}$ are considered in Reuß et al. [F] (see Appx.). The results of the RANS simulation and the hybrid RANS/LES approaches are compared with experimental data in order to investigate the influence of the resolved turbulent scales on the overall representation of the vortex. To evaluate the mean quantities 


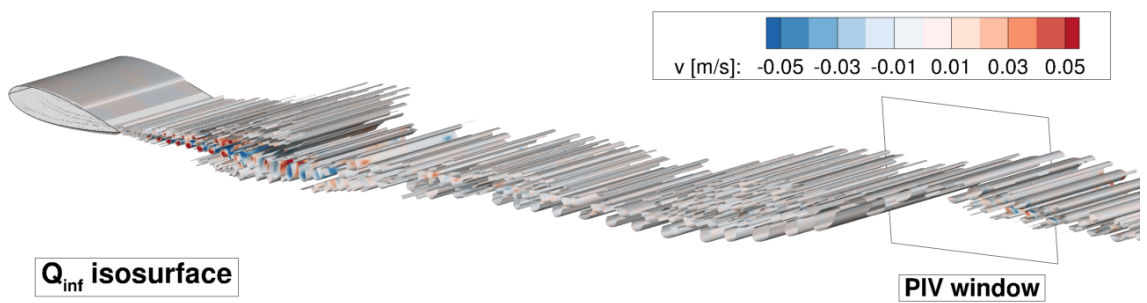

(a) hybrid RANS/LES without forcing

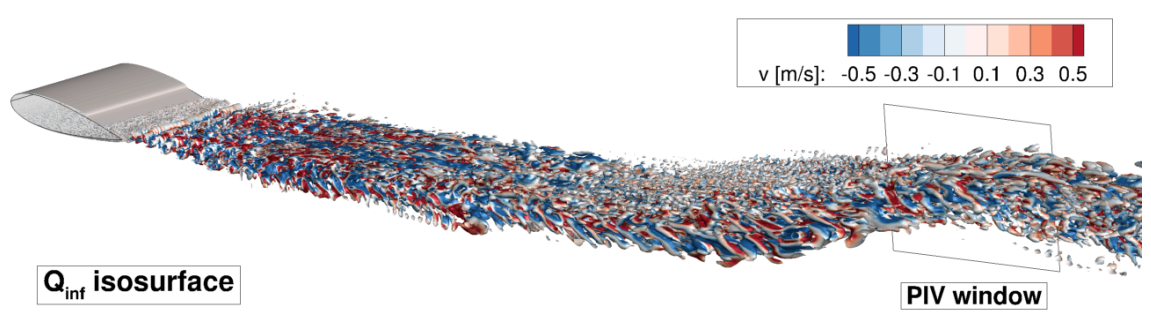

(b) hybrid RANS/LES with forcing

Fig. 4.27: Resolved turbulent structures in the wake of the deflected NACA-0021 airfoil.

in such a time-dependent flow problem, the moving-average formulation is used, see Sect. 2.4. In all simulations in Reuß et al. [F] (see Appx.), similar mean characteristics of the induced disturbance are obtained, which confirms that the main influence of the vortex is represented satisfactorily by the RANS mode. Therefore, the RANS mode is prescribed in the vortex transport region of the target application, while the scale-resolving mode is restricted to the region of the two-element airfoil.

\subsection{Two-element airfoil with vortex interaction}

In order to simulate the interaction of an airfoil-generated vortex with the DLR F15 two-element airfoil, the following strategy is applied: through the rapid deflection of a NACA-0021 airfoil a two-dimensional lateral vortex is generated in the wake, which is convected downstream towards the two-element airfoil at a distance of 3.33 chord lengths. The focus of the investigation lies on the influence of the disturbance on the high-lift airfoil. This case represents the target application for the simulation methods developed in the present thesis. Reference validation data originate from experimental measuring campaigns by Hahn et al. [24] and Klein et al. [32].

\section{Computational setup}

The simulation of the target application utilizes the same grid as the hybrid simulations in Sect. 4.5. The result of the $\mathrm{HRLM}_{\text {opt }}$ simulation serves as initial solution for the target application. In Reuß et al. [E] (see Appx.) it was confirmed that the computational domain can be restricted to the center-line section of the wind tunnel, while Reuß et al. [F] (see Appx.) showed that the RANS method is sufficient to predict the mean characteristics of the transported vortex. The resulting simulation setup is depicted in Fig. 4.28. 


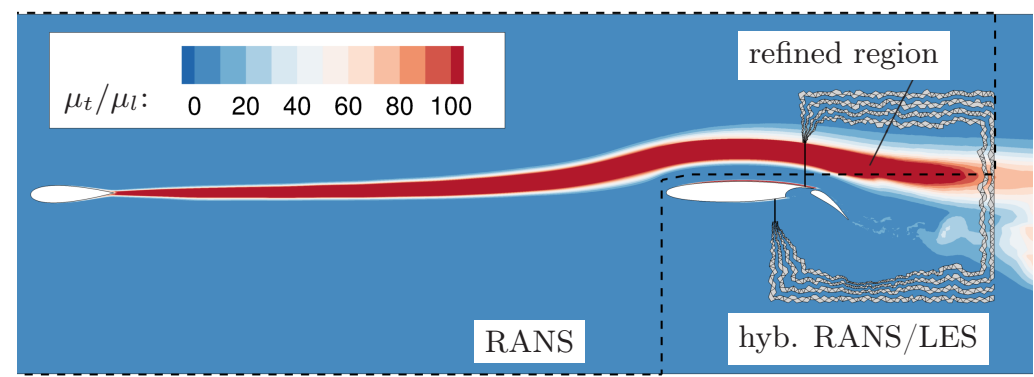

Fig. 4.28: Setup of target application. The RANS region is outlined by the dashed line.

The dashed line outlines the region where RANS mode is prescribed for the vortex transport. The region with a high span-wise resolution, obtained through a local grid refinement, is indicated by the transition elements, see Sect. 3.2.1. Fig. 4.28 depicts the ratio of the modeled eddy viscosity to the laminar viscosity, to illustrate where the RANS model is active.

\section{Results}

The simulation process is described in terms of the non-dimensional time $t^{*}$, which is normalized with the reference time $t_{r e f}=c / u_{\infty}$, i.e. the vortex is convected over the distance of $\Delta x / c=1$ within the time $\Delta t^{*}=1$ in undisturbed flow. The rotation of the NACA0021 airfoil is started at $t^{*}=0$, and full deflection is reached at $t^{*}=2.5$. Fig. 4.29 illustrates the position of the vortex during its transport along the high-lift airfoil, where the image sections visualize different time instances $t^{*}$. The position of the vortex can be identified with the help of the velocity difference vectors. They are computed from the mean velocity field $\langle\boldsymbol{u}\rangle$ at the respective time step and the reference velocity field $\boldsymbol{u}_{\alpha=10^{\circ}}$, obtained with a static, fully deflected vortex-generator airfoil. In the region above the flap, the turbulent flow yields large difference velocities. The color contour depicts the mean-flow velocity.

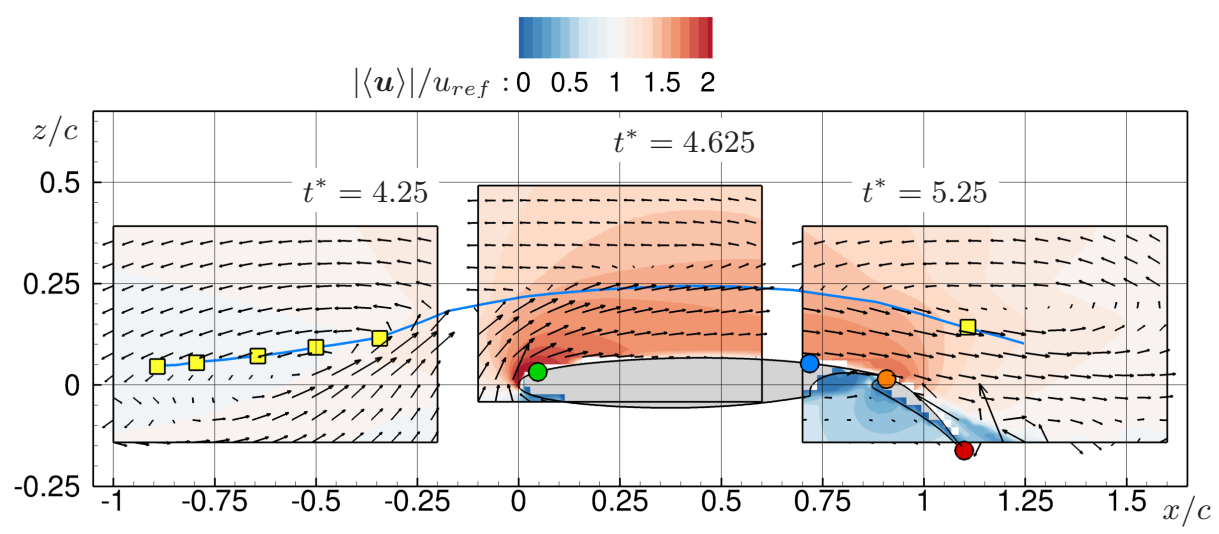

Fig. 4.29: Path of the vortex during its transport along the high-lift airfoil visualized through velocity difference vectors. $\square$ : vortex core extracted from PIV measurements; $\longrightarrow$ extrapolated path.

Upstream of the high-lift airfoil the flow is decelerated through the blockage effect, while the circulation around the high-lift airfoil accelerates the flow above the main 
wing. In Fig. 4.29 the distortion vortex reaches the first position, $x / c \approx-0.5$, upstream of the leading edge at $t^{*}=4.25$. In the second image section, the circulation around the high-lift airfoil stretches the vortex, such that the position of the vortex, $x / c \approx 0.3$, is less sharply defined at $t^{*}=4.625$. In the accelerated flow the vortex is convected over the distance of $\Delta x / c=1.75$ in the time $\Delta t^{*}=1$, such that it reaches the third position, $x / c \approx 1.25$, above the trailing edge of the flap at $t^{*}=5.25$.

The yellow symbols denote the positions of the vortex core as extracted from the PIV measurements by Klein et al. [32]. The blue line indicates the extrapolated path of the vortex core in the region that is not covered by the PIV measurements. In the first position the vortex core in the simulation is located slightly below the blue path, while in the other positions the vortex position is predicted a little farther away from the high-lift airfoil. This can be explained by the findings of Sect. 4.5, where it was shown that no separation at the flap is predicted in the undisturbed case, corresponding to $t^{*}=0$. This leads to a different circulation around the airfoil and consequently to different vortex paths in the simulation and the experiment. However, in principle the simulation method is able to reproduce the vortex transport with satisfactory accuracy, as shown below.

To assess the effect of the vortical disturbance on the aerodynamic performance of the high-lift airfoil, its influence on the pressure distribution is studied in Fig. 4.30.

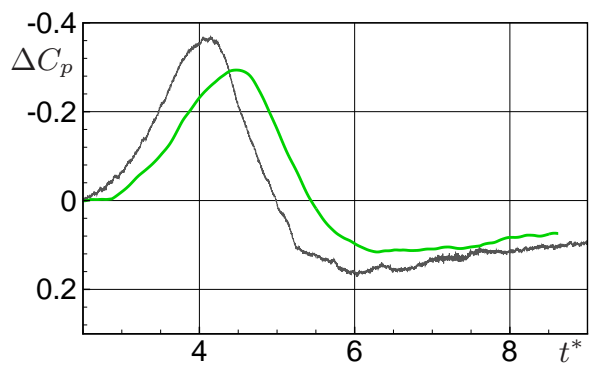

(a) Leading edge of main element

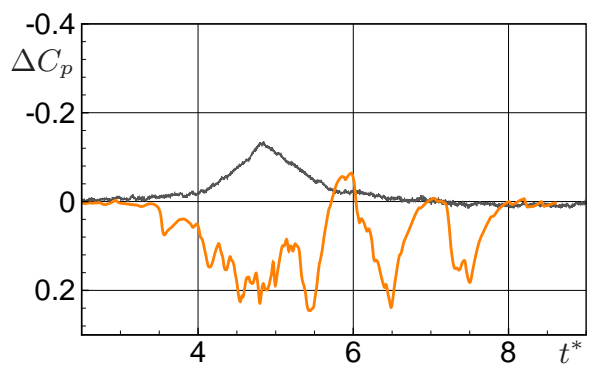

(c) Leading edge of high-lift flap

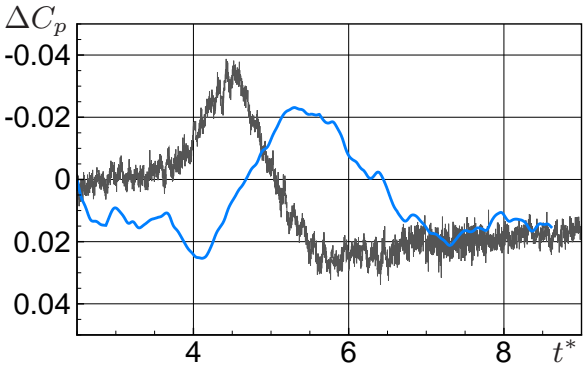

(b) Trailing edge of main element

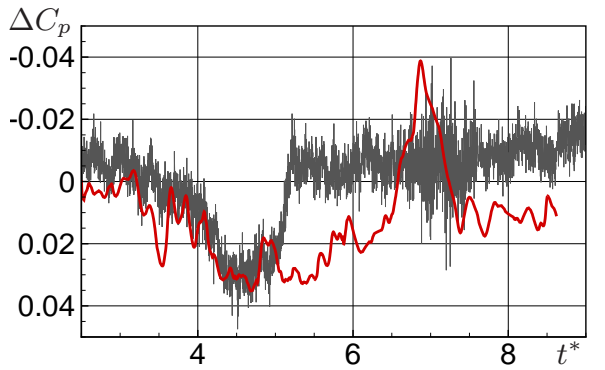

(d) Trailing edge of high-lift flap

Fig. 4.30: Time resolved pressure coefficient $C_{p}$. Comparison of experimental data (black) and simulation results (colored). Note the different scalings of the $y$-axes.

In the experiments, time-resolving signals of the surface pressure were measured at several positions along the main element and the high-lift flap. The colored circles close to the leading and trailing edges of the two elements in Fig. 4.29 indicate four selected measuring positions, in which the numerical results and the experimental data are compared. In Fig. 4.30 the time series of the pressure differences $\Delta C_{p}=C_{p}-$ $C_{p, 0}$ are compared, where $C_{p, 0}$ is the pressure coefficient at $t^{*}=0$. The experimental 


\section{Application of the grid-adaptive algebraic hybrid RANS/LES model}

data represent the ensemble average of more than 80 pitch cycles, while the pressure data from the simulation are obtained with the moving-average formulation, see Sect. 2.4. Note the different scalings of the $y$-axes.

Fig. 4.30 shows that the maximum impact of the passing vortex on the main-element leading edge is predicted at $t^{*}=4.5$, a little later than the measured $t^{*}=4.1$. Similarly, near the main-element trailing edge a maximum is predicted at $t^{*}=5.3$, while it is measured at $t^{*}=4.5$. This may partly be explained by three-dimensional wind-tunnel effects. While Reuß et al. [E] (see Appx.) confirm that the flow about the high-lift airfoil can be considered as two-dimensional, the side walls may influence the transport velocity of the vortex. However, the shape and strength of the induced surface pressure distortion is in reasonable agreement with the experimental data in both measuring stations at the main wing. At the sensor stations on the flap, the surface pressure is influenced by turbulent structures, which are convected from the main-element cove, and the window averaging is not sufficient to eliminate the pressure fluctuations. Therefore, the predicted pressure signal shows a large deviation from the experimental data. It is however not affordable to perform many simulations with slightly different initial flow states, in order to obtain ensemble-averaged data.

In Fig. 4.31 the power spectrum densities of the four time signals are evaluated, to investigate the different scales of the distortion, which occur at the high-lift airfoil. No experimental data are available for comparison. At the two measuring stations at the main wing the influence of the resolved turbulent scales is small and the energy decays quickly. However, at the flap the small structures in the turbulent flow lead to increased energy in the higher frequencies in the red and orange curves.

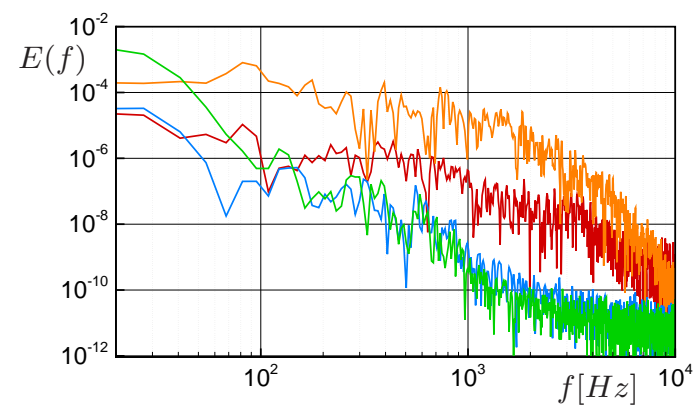

Fig. 4.31: Power spectrum density of the pressure coefficient in the positions of the pressure sensors: (- ${ }^{-}$main-element leading edge; (- - ) main-element trailing

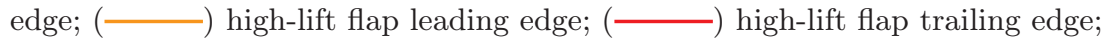

The impact of the vortex on the magnitude of the surface-pressure fluctuations is presented in Fig. 4.32. For this purpose, the difference $\Delta\left\langle C_{p}^{\prime 2}\right\rangle^{1 / 2}=\left\langle C_{p}^{\prime 2}\right\rangle_{t^{*}}^{1 / 2}-$ $\left\langle C_{p}^{\prime 2}\right\rangle_{0}^{1 / 2}$ is computed from the variance of the pressure coefficient $C_{p}$, where $\left\langle C_{p}^{\prime 2}\right\rangle_{0}^{1 / 2}$ denotes the value at $t^{*}=0$. No reference data are available from the experiment. The mean values are computed with the moving-average formulation, c.f. Sect. 2.4. Therefore, the time dependence of the mean values is preserved, and the influence of the passing vortex decays after a while.

The red curve in Fig. 4.32 represents the flow near the maximum impact of the vortex on main-wing leading edge at the time $t^{*}=4.25$. In (a) it can be clearly seen, that the vortex increases the pressure fluctuations at the leading edge of the main wing. The blue curve represents the flow near the maximum impact of the vortex 


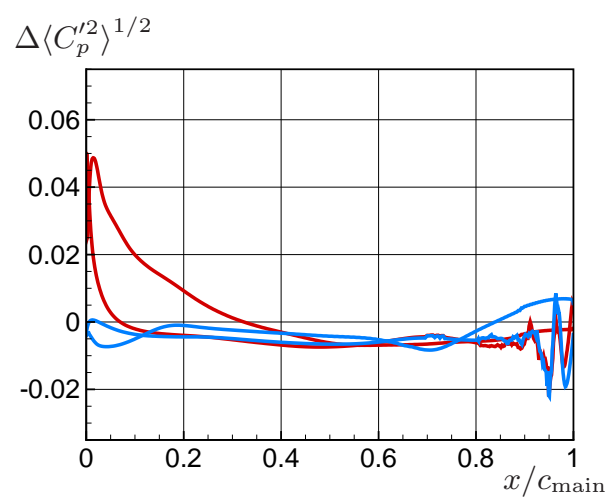

(a) Main wing

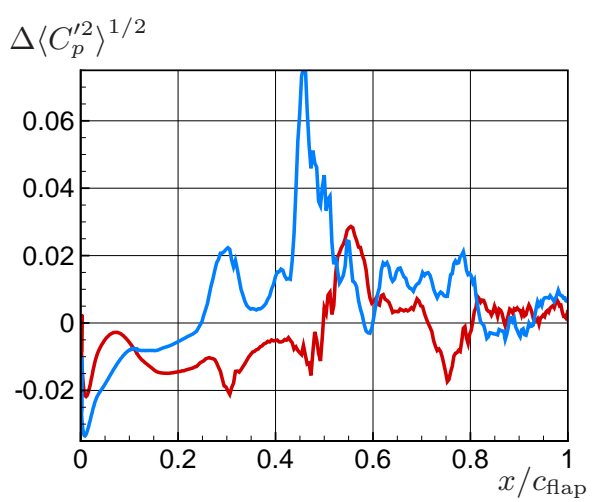

(b) High-lift flap

Fig. 4.32: Impact of the passing vortex on the pressure fluctuations at the two-element airfoil: $t^{*}=4.25$ $t^{*}=5.25$.

on the flap leading edge at the time $t^{*}=5.25$. At this time instance the pressure fluctuations at the main wing have decayed, and in (b) a maximum of the pressure fluctuations can be observed at the flap.

In summary, the computation of the target application of the present thesis confirms that the presented simulation strategy is able to reproduce the experimental data with satisfactory results. The vortex is preserved during the transport towards the high-lift airfoil, and the essential effects are well predicted. The computed impact of the vortical disturbance on the surface pressure broadly agrees with that seen in the measurements. 



\section{Conclusion}

The present thesis aims to provide a reliable simulation strategy for high-lift applications under disturbed inflow conditions. As a consequence of the known weaknesses of RANS turbulence models in flows with massive separation, a hybrid RANS/LES method was chosen as base-line approach: the Algebraic Delayed DES (ADDES), first introduced by Knopp et al. [35]. In the original version of the ADDES, algebraic sensors evaluate the boundary-layer velocity profiles to assess the state of the flow and adjust the model behavior. In the present thesis the ADDES was extended, improved, and validated for several fundamental flow cases and application challenges.

To start with, the wall-modeling capabilities of the IDDES were included in the ADDES. For its automatic activation, another algebraic sensor was developed, which is able to detect wall-modeled LES mode. The algebraic sensors of the ADDES were successfully utilized in simulations of the flow over a wall-mounted hump and the flow over a two-element airfoil.

Moreover, the present thesis addresses the grey-area problem, which occurs at RANSto-LES model interfaces. In order to reduce the amount of modeled turbulent kinetic energy, an improved vorticity-based LES filter width by Chauvet et al. [9] was adopted and reformulated for the unstructured dual-grid approach. Additionally, a combination of the vorticity-based filter with the IDDES was proposed. The improved filter was compared to the standard filter for wall-modeled LES simulations of a turbulent channel flow, and consistent results were obtained. Furthermore, for the flow over a backward-facing step the generation of resolved turbulent structures was accelerated and the grey area was strongly reduced. For the flow over a two-element high-lift airfoil, considerably smaller turbulent structures were resolved with the vorticity-based filter width.

An optimized numerical scheme with low-dissipation properties, see Probst and Reuß [D], and low-dispersion properties, see Löwe et al. [42], was utilized to improve the results of the hybrid RANS/LES simulations. This scheme was considered in combination with wall-modeled LES of turbulent channel flows at the Reynolds numbers of $R e_{\tau}=1100$ and $R e_{\tau}=4200$, and consistent results were obtained.

To enable a smooth transition from LES to RANS regions, sponge sources, which are able to transfer resolved turbulent structures into modeled turbulence, were assessed for a turbulent channel flow. Near the inflow boundary, synthetic resolved structures were introduced using the STG method by Adamian and Travin [3], which was implemented and improved in the framework of the ADDES by François and Radespiel [17]. The synthetic turbulence together with the sponge terms were shown to allow a rapid transition between RANS and LES in both directions. However, the implementation should be extended to consider reference values from farther upstream for the sponge terms. Besides, synthetic turbulence was applied to the test case of a two-element airfoil, where it lead to the desired rapid breakup the wake flow.

An important issue in scale-resolving approaches is the grid resolution. In order to obtain reliable results, it is necessary to ensure a sufficient resolution of the turbulent structures in the LES regions. Therefore, a grid-resolution sensor was developed, which evaluates the capability of the grid to resolve the relevant turbulent length scales. This sensor can be used as input for an automatic grid refinement of the insufficiently resolved regions. For the test case of the backward facing step the sensor was successfully evaluated and the results were used for a local grid refinement. For the test case of the two-element airfoil, the sensors was evaluated and confirmed that 
the grid was sufficiently fine in the LES region.

In the target application of the present thesis, the interaction of a generic airfoilgenerated vortex with a two-element high-lift airfoil was investigated. To simulate the generation of the onflow disturbances, the grid deformation method of the DLRTAU code was chosen. Furthermore, a simulation strategy to transport the vortex over the large distance between the vortex-generator airfoil and the high-lift airfoil was presented: it was demonstrated, that the RANS mode is able to accurately represent the relevant scales and mean-flow characteristics of the vortex. Finally, in the target application all numerical and modeling improvements of the simulation method were combined. The numerical results were in reasonable agreement with the experimental data and the mean influence of the disturbance on the high-lift airfoil was successfully reproduced. However, in the simulations the flow at the flap was attached, while the experiments indicate separation on the flap. This problem should be scrutinized in future applications, e.g. synthetic turbulence could be introduced in the boundary layer on the flap. During the development of the improved simulation strategy, intermediate results were published at several work stages. The publications are attached in the appendix of this thesis.

In the presented applications, only little use was made of the automatization capabilities of the ADDES. For example, the location of the inflow region for the synthetic turbulent structures was fixed in advance. However, one of the strengths of the algebraic sensors in the ADDES is the possibility to detect different flow states: the flexible data structure of the wall-normal lines allows the parallel real-time evaluation of boundary-layer velocity profiles even for complex flow cases, which comprise three-dimensional flow phenomena. This means on the one hand, that the boundary layer sensors are able to adjust the model behavior to automatically select the suited simulation approach, i.e. RANS, wall-modeled LES, or LES, respectively. On the other hand, the grid resolution can be adapted, as well: during the entire simulation, the grid is assessed through the grid-resolution sensor, and if an underresolved flow region is identified, the grid will be automatically refined in this region. This automatization should be exploited and carefully assessed in future applications. 


\section{References}

[1] M. Abate. Gust boundary condition for the TAU-Code. Technical report, IB 124-2007/9, DLR, Braunschweig, Germany, 2007.

[2] A. Abbas-Bayoumi and K. Becker. An industrial view on numerical simulation for aircraft aerodynamic design. Journal of Mathematics in Industry, 1(10), 2011.

[3] D. Y. Adamian and A. K. Travin. An Efficient Generator of Synthetic Turbulence at RANS-LES Interface in Embedded LES of Wall-Bounded and Free Shear Flows. In A. Kuzmin (ed.): Computational Fluid Dynamics 2010, pp. 739-744. Springer, 2011.

[4] T. Alrutz and M. Orlt. Parallel Dynamic Grid Refinement for Industrial Applications. In P. Wesseling et al. (eds.): European Conference on Computational Fluid Dynamics (ECCOMAS CFD), TU Delft, The Netherlands, 2006.

[5] A. Brandt. Multigrid Techniques: 1984 Guide with Applications to Fluid Dynamics. VKI Lecture series 1984-04, 1984.

[6] L. Castillo, X. Wang, and W. K. George. Separation Criterion for Turbulent Boundary Layers Via Similarity. Journal of Fluids Engineering, 126(3):297-304, 2004.

[7] R.-D. Cécora, R. Radespiel, B. Eisfeld, and A. Probst. Differential ReynoldsStress Modeling for Aeronautics. AIAA Journal, 53(3):739-755, 2015.

[8] I. B. Celik, Z. N. Cehreli, and I. Yavuz. Index of Resolution Quality for Large Eddy Simulations. Journal of Fluids Engineering, 127(5):949-958, 2005.

[9] N. Chauvet, S. Deck, and L. Jacquin. Zonal Detached Eddy Simulation of a Controlled Propulsive Jet. AIAA Journal, 45(10):2458-2473, 2007.

[10] H. Choi and P. Moin. Grid-Point Requirements for large eddy simulation: Chapman's estimates revisited. Center for Turbulence Research, Annual Research Briefs, pp. 31-36, 2011.

[11] F. K. Chow, R. L. Street, M. Xue, and J. H. Ferziger. Explicit Filtering and Reconstruction Turbulence Modeling for Large-Eddy Simulation of Neutral Boundary Layer Flow. Journal of Atmospheric sciences, 62:2058-2077, 2005.

[12] G. Dargel, H. Hansen, J. Wild, T. Streit, H. Rosemann, and K. Richter. Aerodynamische Flügelauslegung mit multifunktionalen Steuerflächen. In DGLR Jahrbuch 2002. DGLR, vol. 1, pp. 1605-1615, ISSN 00704083, 2002.

[13] S. Deck. Recent improvements in the Zonal Detached Eddy Simulation (ZDES) formulation. Theoretical and Computational Fluid Dynamics, 26(6):523-550, 2011.

[14] D. M. Driver and H. L. Seegmiller. Features of a Reattaching Turbulent Shear Layer in Divergent Channel Flow. AIAA Journal, 23(2):163-171, 1985.

[15] F. Ducros, F. Nicoud, and T. Poinsot. Wall-Adapting Local Eddy-Viscosity models for simulations in complex geometries. In M. Baines (ed.): Numerical Methods in Fluid Dynamics IV. Oxford, UK, 1998. 
[16] R. P. Dwight. Time-Accurate Navier-Stokes Calculations with Approximately Factored Implicit Schemes. In C. Groth et al. (eds.): Computational Fluid Dynamics 2004, pp. 211-217. Springer, 2004.

[17] D. G. François and R. Radespiel. Airfoil Stall Simulations with Algebraic Delayed DES and Physically Based Synthetic Turbulence for RANS-LES Transition. AIAA 2014-2574, 2014.

[18] D. G. François, R. Radespiel, S. Reuß, and A. Probst. Computations of Separated Flows with Hybrid RANS/LES Approaches. In R. Radespiel et al. (eds.): Advances in Simulation of Wing and Nacelle Stall, Notes on Numerical Fluid Mechanics and Multidisciplinary Design, Vol.131. Springer, 2015.

[19] J. Fröhlich. Large Eddy Simulation turbulenter Strömungen. Teubner, 2006.

[20] J. Fröhlich and D. von Terzi. Hybrid LES/RANS Methods for the Simulation of Turbulent Flows. Progress in Aerospace Sciences, 44(5):349-377, 2008.

[21] K. Geurts and A. Probst. Hybrid RANS-LES Models. In N. Kroll et al. (eds.): Computational Flight Testing, Notes on Numerical Fluid Mechanics and Multidisciplinary Design, Vol. 123. Springer, 2013.

[22] S. S. Girimaji. Partially-Averaged Navier-Stokes Model for Turbulence: A Reynolds-Averaged Navier-Stokes to Direct Numerical Simulation Bridging Method. Journal of Applied Mechanics, 73(3):413-421, 2006.

[23] D. Greenblatt, K. B. Paschal, C.-S. Yao, J. Harris, N. W. Schaeffler, and A. E. Washburn. A Separation Control CFD Validation Test Case Part 1: Baseline \& Steady Suction. AIAA 2004-2220, 2004.

[24] D. Hahn, P. Scholz, and R. Radespiel. Vortex generation in a low speed wind tunnel and vortex interactions with a high-lift airfoil. AIAA 2012-3024, 2012.

[25] R. Heinrich, L. Reimer, and A. Michler. Multidisciplinary simulation of maneuvering aircraft interacting with atmospheric effects using the DLR TAU code. In RTO AVT-189 Specialists' Meeting on Assessment of Stability and Control Prediction Methods for Air and Sea Vehicles, Oct. 12-14, Portsdown West, UK, 2011.

[26] C. Helmke, T. Auerswald, S. Raasch, and J. Bange. Comparison of two methods to provide highly resolved atmospheric turbulence data for simulations of wing and nacelle circulations. In Joint Symposium "Simulation of Wing and Nacelle Stall", Jun. 22-23, 2010, Braunschweig, Germany, 2010.

[27] J. C. R. Hunt, A. A. Wray, and P. Moin. Eddies, Streams, and Convergence Zones in Turbulent Flows. Center for Turbulence Research, Proceedings of the Summer Program, 1988.

[28] S. Jakirlić and K. Hanjalić. A new approach to modelling near-wall turbulence energy and stress dissipation. Journal of Fluid Mechanics, 459:139-166, 2002.

[29] N. Jarrin, S. Benhamadouche, D. Laurence, and R. Prosser. A synthetic-eddymethod for generating inflow conditions for large-eddy simulations. International Journal of Heat and Fluid Flow, 27:585-593, 2006.

[30] I. Kim, S. Elghobashi, and W. Sirignano. Unsteady Flow Interactions Between a Pair of Advected Vortex Tubes and a Rigid Sphere. International Journal of Multiphase Flow, 23(1):1-23, 1997. 
[31] M. Klein. An Attempt to Assess the Quality of Large Eddy Simulations in the Context of Implicit Filtering. Flow, Turbulence and Combustion, 75:131-147, 2005.

[32] S. Klein, D. Hoppmann, P. Scholz, and R. Radespiel. High-Lift Airfoil Interacting with a Vortical Disturbance: Wind-Tunnel Measurements. AIAA Journal, 53(6):1681-1692, 2015.

[33] C. Knigge. Development and improvement of two methods of different complexity to simulate atmospheric boundary layer turbulence for aircraft design studies. In R. Radespiel et al. (eds.): Advances in Simulation of Wing and Nacelle Stall, Notes on Numerical Fluid Mechanics and Multidisciplinary Design, Vol.131. Springer, 2015.

[34] T. Knopp and A. Probst. An Algebraic Sensor for the RANS-LES Switch in Delayed Detached-Eddy Simulation. In A. Dillmann et al. (eds.): New Results in Numerical and Experimental Fluid Mechanics VIII, Notes on Numerical Fluid Mechanics and Multidisciplinary Design, Vol. 121, pp. 457-464. Springer, 2013.

[35] T. Knopp, X. Zhang, R. Kessler, and G. Lube. Enhancement of an industrial finite-volume code for large-eddy-type simulation of incompressible high Reynolds number flow using near-wall modelling. Computer Methods in Applied Mechanics and Engineering, 199(13-16):890-902, 2010.

[36] J. C. Kok. A high-order low-dispersion symmetry-preserving finite-volume method for compressible flow on curvilinear grids. Journal of Computational Physics, 228(18):6811-6832, 2009.

[37] J. C. Kok and H. van der Ven. Destabilizing free shear layers in X-LES using a stochastic subgrid-scale model. In S.-H. Peng et al. (eds.): Progress in Hybrid RANS-LES Modelling, Notes on Numerical Fluid Mechanics and Multidisciplinary Design, Vol. 111, pp. 179-189. Springer, 2009.

[38] D. J. J. Leclercq and C. J. Doolan. The interaction of a bluff body with a vortex wake. Journal of Fluids and Structures, 25(5):867-888, 2009.

[39] M. Lee and R. D. Moser. Direct numerical simulation of turbulent channel flow up to $\operatorname{Re}_{\tau}=5200$. Journal of Fluid Mechanics, 774:395-415, 2015.

[40] M. Lesieur and O. Métais. New trends in large-eddy simulations of turbulence. Annual Review of Fluid Mechanics, 28:45-82, 1996.

[41] D. K. Lilly. The Representation of Small-Scale Turbulence in Numerical Simulation Experiments. NCAR Manuskript No. 281, 1966.

[42] J. Löwe, A. Probst, T. Knopp, and R. Kessler. A Low-Dissipation LowDispersion Second-Order Scheme for Unstructured Finite-Volume Flow Solvers. AIAA 2015-0815, 2015.

[43] A. Lozano-Durán and J. Jiménez. Effect of the computational domain on direct simulations of turbulent channels up to $\mathrm{Re}_{\tau}=4200$. Physics of Fluids, 26 (011702), 2014.

[44] A. Madrane, A. Raichle, and A. Stuermer. Parallel Implementation of a Dynamic Overset Unstructured Grid Approach. In P. Neittaanmäki et al. (eds.): European Conference on Computational Fluid Dynamics (ECCOMAS CFD), Jyväskylä, Finnland, 2004. 
[45] F. R. Menter. Zonal Two Equation k-w Turbulence Models for Aerodynamic Flows. AIAA 1993-2906, 1993.

[46] F. R. Menter and M. Kuntz. Adaptation of Eddy-Viscosity Turbulence Models to Unsteady Separated Flow Behind Vehicles. In R. McCallen et al. (eds.): The Aerodynamics of Heavy Vehicles: Trucks, Buses, and Trains, Lecture Notes in Applied and Computational Mechanics. Springer, 2004.

[47] F. R. Menter, M. Kuntz, and R. Bender. A Scale-Adaptive Simulation Model for Turbulent Flow Predictions. AIAA 2003-0767, 2003.

[48] F. R. Menter, M. Kuntz, and R. Langtry. Ten Years of Industrial Experience with the SST Turbulence Model. In K. Hanjalić et al. (eds.): Turbulence, Heat and Mass Transfer 4, pp. 625-632. Begell House, 2003.

[49] C. R. Mockett, M. Fuchs, A. V. Garbaruk, M. L. Shur, P. R. Spalart, M. K. Strelets, F. Thiele, and A. K. Travin. Two Non-zonal Approaches to Accelerate RANS to LES Transition of Free Shear Layers in DES. In S. Girimaji et al. (eds.): Progress in Hybrid RANS-LES Modelling, Notes on Numerical Fluid Mechanics and Multidisciplinary Design, Vol. 130, pp. 187-201. Springer, 2015.

[50] C. R. Mockett, W. Haase, and F. Thiele. Go4Hybrid: A European Initiative for Improved Hybrid RANS-LES Modelling. In S. Girimaji et al. (eds.): Progress in Hybrid RANS-LES Modelling, Notes on Numerical Fluid Mechanics and Multidisciplinary Design, Vol. 130, pp. 299-303. Springer, 2015.

[51] P. Moin and K. Mahesh. Direct Numerical Simulation: A Tool in Turbulence Research. Annual Review of Fluid Mechanics, 30:539-578, 1998.

[52] R. D. Moser, J. Kim, and N. N. Mansour. Direct numerical simulation of turbulent channel flow up to $\operatorname{Re}_{\tau}=590$. Physics of Fluids, 11(4):943-945, 1999.

[53] J. W. Naughton, S. Viken, and D. Greenblatt. Skin-Friction Measurements on the NASA Hump Model. AIAA Journal, 44(6):1255-1265, 2006.

[54] F. Nicoud and F. Ducros. Subgrid-scale stress modelling based on the square of the velocity gradient tensor. Flow, Turbulence and Combustion, 62(3):183-200, 1999.

[55] F. Nicoud, H. Baya Toda, O. Cabrit, S. Bose, and J. Lee. Using singular values to build a subgrid-scale model for Large Eddy Simulations. Physics of Fluids, 23(8):085106, 2011.

[56] N. V. Nikitin, F. Nicoud, B. Wasistho, K. D. Squires, and P. R. Spalart. An approach to wall modeling in large-eddy simulations. Physics of Fluids, 12(7): $1629-1632,2000$.

[57] M. Pamiès, P.-E. Weiss, E. Garnier, S. Deck, and P. Sagaut. Generation of synthetic turbulent inflow data for large eddy simulation of spatially evolving wall-bounded flows. Physics of Fluids, 21(4):045103, 2009.

[58] U. Piomelli and J. R. Chasnov. Large-Eddy Simulations: Theory and Applications. In M. Hallbäck et al. (eds.): Turbulence and Transition Modelling, ERCOFTAC Series, pp. 263-336. Springer, 1996.

[59] U. Piomelli, P. Moin, and J. H. Ferziger. Model consistency in large eddy simulation of turbulent channel flows. Physics of Fluids, 31(7):1884-1891, 1988. 
[60] S. B. Pope. Turbulent Flows. Cambridge University Press, 2000.

[61] A. Probst. Reynoldsspannungsmodellierung für das Überziehen in der Flugzeugaerodynamik. PhD thesis, TU Braunschweig, 2013.

[62] A. Probst and S. Reuß. Scale-Resolving Simulations of Wall-Bounded Flows with an Unstructured Compressible Flow Solver. In S. Girimaji et al. (eds.): Progress in Hybrid RANS-LES Modelling, Notes on Numerical Fluid Mechanics and Multidisciplinary Design, Vol. 130, pp. 481-491. Springer, 2015.

[63] A. Probst, R. Radespiel, C. Wolf, T. Knopp, and D. Schwamborn. A Comparison of Detached-Eddy Simulation and Reynolds-Stress Modelling Applied to the Flow over a Backward-Facing Step and an Airfoil at Stall. AIAA 2010-0920, 2010.

[64] A. Probst, J. Löwe, S. Reuß, T. Knopp, and R. Kessler. Scale-Resolving Simulations with a Low-Dissipation Low-Dispersion Second-Order Scheme for Unstructured Finite-Volume Flow Solvers. AIAA 2015-0816, 2015.

[65] R. Radespiel, E. Turkel, and N. Kroll. Assessment of Preconditioning Methods. Technical report, FB 95-29, DLR, Braunschweig, Germany, 1995.

[66] C. L. Rumsey and S. X. Ying. Prediction of high lift: review of present CFD capability. Progress in Aerospace Sciences, 38(2):145-180, 2002.

[67] P. Schlatter, Q. Li, G. Brethouwer, A. V. Johansson, and D. S. Henningson. Simulations of spatially evolving turbulent boundary layers up to $\operatorname{Re}_{\theta}=4300$. International Journal of Heat and Fluid Flow, 31(3):251-261, 2010.

[68] H. Schlichting and K. Gersten. Grenzschicht-Theorie. Springer, 10th edition, 2006 .

[69] P. Scholz, C. J. Kähler, R. Radespiel, J. Wild, and G. Wichmann. Active Control of Leading-Edge Separation within the German Flow Control Network. AIAA 2009-0529, 2009.

[70] D. Schwamborn, T. Gerhold, and R. Heinrich. The DLR TAU-Code: Recent Applications in Reaserach and Industry. In P. Wesseling et al. (eds.): European Conference on Computational Fluid Dynamics (ECCOMAS CFD), TU Delft, The Netherlands, 2006.

[71] M. L. Shur, P. R. Spalart, M. K. Strelets, and A. K. Travin. A hybrid RANS-LES approach with delayed-DES and wall-modelled LES capabilities. International Journal of Heat and Fluid Flow, 29(6):1638-1649, 2008.

[72] M. L. Shur, P. R. Spalart, M. K. Strelets, and A. K. Travin. A Rapid and Accurate Switch from RANS to LES in Boundary Layers Using an Overlap Region. Flow, Turbulence and Combustion, 86:179-206, 2011.

[73] J. Smagorinsky. General Circulation Experimets with the Primitive Equations. Monthly weather review, 91(3):99-164, 1963.

[74] P. R. Spalart. Young-Person's Guide to Detached-Eddy Simulation Grids. Technical report, CR-2001-211032, NASA Langley Research Center, 2001.

[75] P. R. Spalart. Detached-Eddy Simulation. Annual Review of Fluid Mechanics, 41:181-202, 2009. 
[76] P. R. Spalart and S. R. Allmaras. A One-Equatlon Turbulence Model for Aerodynamic Flows. AIAA 1992-0439, 1992.

[77] P. R. Spalart, W. H. Jou, M. K. Strelets, and S. R. Allmaras. Comments on the Feasibility of LES for Wings, and on a Hybrid RANS/LES Approach. In C. Liu et al. (eds.): Advances in DNS/LES, pp. 137-147. Greyden Press, 1997.

[78] P. R. Spalart, S. Deck, M. L. Shur, K. D. Squires, M. K. Strelets, and A. K. Travin. A new version of detached-eddy simulation, resistant to ambiguous grid densities. Theoretical and Computational Fluid Dynamics, 20:181-195, 2006.

[79] H. W. Stock and W. Haase. The Determination of Turbulent Length Scales in Algebraic Turbulence Models for Attached and Slightly Separated Flows Using Navier-Stokes Methods. AIAA 1987-1302, 1987.

[80] S. Stolz, N. A. Adams, and L. Kleiser. An approximate deconvolution model for large-eddy simulation with application to incompressible wall-bounded flows. Physics of Fluids, 13(4):997-1015, 2001.

[81] M. K. Strelets. Detached Eddy Simulation of Massively Separated Flows. AIAA 2001-0879, 2001.

[82] M. Svärd, J. Lundberg, and J. Nordström. A computational study of vortexairfoil interaction using high-order finite difference methods. Computers \&f Fluids, 39(8):1267-1274, 2010.

[83] G. R. Tabor and M. H. Baba-Ahmadi. Inlet conditions for large eddy simulation: A review. Computers \& Fluids, 39(4):553-567, 2010.

[84] H. Tennekes and J. L. Lumley. A First Course in Turbulence. The MIT Press, 1972 .

[85] A. K. Travin, M. L. Shur, P. R. Spalart, and M. K. Strelets. Improvement of Delayed Detached-Eddy Simulation for LES with Wall Modelling. In P. Wesseling et al. (eds.): European Conference on Computational Fluid Dynamics (ECCOMAS CFD), TU Delft, The Netherlands, 2006.

[86] E. Turkel. Preconditioning-Squared Methods for Multidimensional Aerodynamics. AIAA 1997-2025, 1997.

[87] K. P. Wawrzinek, T. Lutz, and E. Krämer. Numerical Studies of Turbulent Flow Influence on a Two-Element Airfoil. In R. Radespiel et al. (eds.): Advances in Simulation of Wing and Nacelle Stall, Notes on Numerical Fluid Mechanics and Multidisciplinary Design, Vol.131. Springer, 2015.

[88] F. M. White. Fluid Mechanics. McGraw Hill, 1998.

[89] D. C. Wilcox. Turbulence Modeling for CFD. DCW Industries, 1994.

[90] J. Wild, M. Pott-Pollenske, and B. Nagel. An integrated design approach for low noise exposing high-lift devices. AIAA 2005-2843, 2005.

[91] R. Wokoeck, N. Krimmelbein, J. Ortmanns, V. Ciobaca, R. Radespiel, and A. Krumbein. RANS Simulation and Experiments on the Stall Behaviour of an Airfoil with Laminar Separation Bubbles. AIAA 2006-0244, 2006.

[92] C. Wolf. A Chimera Simulation Method and Detached Eddy Simulation for Vortex-Airfoil Interactions. PhD thesis, Georg-August-Universität Göttingen, 2010. 
[93] C. Wolf, A. Raichle, T. Knopp, and D. Schwamborn. Chimera Simulations of Transported Large-Scale Vortices and their Interaction with Airfoils. In A. Dillmann et al. (eds.): New Results in Numerical and Experimental Fluid Mechanics VII, Notes on Numerical Fluid Mechanics and Multidisciplinary Design, Vol. 112, pp. 117-124. Springer, 2010. 



\title{
Chimera technique for transporting disturbances
}

\author{
S. Reuß ${ }^{1}$, C. Wolf ${ }^{1}$, T. Knopp ${ }^{1}$, A. Raichle ${ }^{2}$, D. Schwamborn ${ }^{1}$ \\ ${ }^{1}$ Institute of Aerodynamics and Flow Technology, \\ DLR (German Aerospace Center) Göttingen, Germany \\ ${ }^{2}$ Institute of Aerodynamics and Flow Technology, \\ DLR (German Aerospace Center) Braunschweig, Germany
}

Published in:

International Journal for Numerical Methods in Fluids, Volume 70, Issue 12, pp. 1558-1572, 2012

\begin{abstract}
The Chimera technique for moving grids is used to take into account nonhomogeneous unsteady inflow conditions in the simulation of aerodynamic flows. The method is applied to simulate the transport of a large-scale vortex by a mean velocity field over a large distance, where it finally interacts with an airfoil. The Chimera approach allows one to resolve the vortex on a fine grid, whereas the unstructured background grid covering most of the computational domain can be much coarser. This method shows the same low numerical dissipation as a simulation on a globally fine grid. Several precursor tests are performed with a finite modified analytical Lamb-Oseen type vortex, to study the influence of spatial and temporal resolution and the employed numerical scheme. Then the interaction of an analytical vortex with a NACA0012 airfoil and with an ONERA-A airfoil near stall is studied. Finally a realistic vortex is generated by a ramping airfoil and is transported on a moving Chimera block and then interacts with a two-element airfoil, which allows one to simulate a typical setup for a gust generator in aerodynamic facilities.
\end{abstract}

\section{Introduction}

The weather is one reason for airplane accidents. Of particular interest are nonhomogeneous unsteady onflow conditions, consisting of large-scale vortices due to 
atmospheric conditions. The focus is on the influence of the disturbance in the onflow on the flow field around the body and on the corresponding aerodynamic coefficients of lift, drag and moment. The prediction of the impact of a discrete symmetrical vertical and lateral gust is part of the current certification process of an airplane. According to the certification specification EASA CS 25.341 the shape of the gust must be taken as

$$
U=\frac{U_{d s}}{2}\left[1-\cos \left(\frac{\pi s}{H}\right)\right] \quad \text { for } \quad 0 \leq 2 s \leq H,
$$

where $s$ is the distance penetrated into the gust, $U_{d s}$ is the design gust velocity and $H$ is the gust gradient.

The size of atmospheric vortices is typically more than one order of magnitude larger than the size of the aerodynamic body. Many studies of vortex body interactions have been performed, but often the vortices are of the same size as the body that is subjected to them. In [1] the interaction of a tail rotor blade of a helicopter with a vortex generated by the main rotor is investigated experimentally and numerically. The interaction of a vortex wake with a bluff body is investigated in [2]. Additionally to the effect on the aerodynamic forces the noise caused by the collision is studied.

In aerodynamic simulations the farfield boundary is typically at a distance of one hundred chord lengths from the aerodynamic body, e.g., an airfoil, a wing or an airplane. Large-scale vortices in the inflow conditions need to be brought into the flow field at a sufficiently large distance from the body in order to avoid a disturbance of the flow around the body due to elliptic propagation of information. The vortex needs to be resolved on a sufficiently fine mesh all the way to the airfoil when it is convected with the onflow velocity. This method has been used in [3] where a boundary condition is described that allows one to introduce a sinusoidal disturbance, as described in equation (1), to a flow field. It is applied to predict the impact on a NACA0012 airfoil. Otherwise, if the grid is not fine enough, the shape and strength of the vortex can be altered in an undesired way due to numerical dissipation. In [4] higher-order methods are used to preserve the vortex during the interaction with a NACA0012 airfoil. However, industrial CFD solvers are usually based on second order methods on unstructured meshes. A global mesh refinement is often unfeasible due to the very large additional numerical costs. A second approach is a timeaccurate local grid refinement and derefinement [5]. This has the potential drawback that poorly shaped nonisotropic elements are introduced which can lead to an increase in numerical dissipation and hence to a detoriation of the vortex.

As a remedy, this paper presents a Chimera-based approach, that was first used in $[6,7]$ and later in $[8,9,10]$.

The idea of the present work is to transport the vortex on a fine grid which is moving at the same speed as the vortex, whereas the mesh around the body is fine only in the vicinity of the body and becomes much coarser with increasing distance from the body. The Chimera mesh for the vortex is built of hexahedral elements which ensures a low numerical dissipation. In case that the vortex interacts with a body, the Chimera grid is stopped at a certain distance from the body where the grid around the body is fine enough. Then the vortex is convected from the Chimera mesh onto the aerodynamic body mesh and the vortex interacts with the flow around the body.

In the present paper we use analytical and generic vortices in the inflow conditions. In future work, we plan to consider atmospherically realistic turbulence in the onflow.

International Journal for Numerical Methods in Fluids, 70(12) pp. 1558-1572, 2012 
The paper is organized as follows: In section 2 the DLR TAU-code is described, the details of the implementation of the Chimera technique are given in section 3 . In the subsequent section 4 first the required numerical scheme, grid resolution and time step size to maintain a finite analytical vortex at rest and during the transport with a moving Chimera block are determined. Then the interaction of an analytical vortex with an NACA0012 and an ONERA-A profile is studied. Finally a vortex is generated by a rapidly deflected NACA0012 profile and the interaction with a two-element airfoil is investigated. A conclusion is given in section 5 .

\section{The DLR TAU-code}

The DLR TAU-code [11] is an unstructured finite volume solver for the compressible Navier-Stokes equations (NSE). We consider the computational domain $\Omega \subset \mathbb{R}^{d}$, $d=2,3$, discretized with a grid. The control volumes $V_{i}$, the so called dual mesh cells, are associated with every grid node and are constructed during the preprocessing. This allows the use of mixed cell types in the primary grid, and thus of structured as well as unstructured meshes and hybrid meshes, that comprise structured and unstructured regions. The dual mesh can be interpreted as $\bar{\Omega}=\cup_{i} \overline{V_{i}}$, where the overline denotes the closure of a domain. We seek the density $\rho(\mathbf{x}, t)$, the velocity vector $\mathbf{u}(\mathbf{x}, t)$ and the temperature $T(\mathbf{x}, t)$ with $(\mathbf{x}, t) \in \Omega \times(0, \infty)$. The cell averages $\mathbf{W}_{i}$ of the conservative variables are stored in the centers of the dual cells:

$$
\mathbf{W}_{i}=\frac{1}{\operatorname{vol}\left(V_{i}\right)} \int_{V_{i}} \mathbf{w}_{i} d V \quad \text { and } \quad \mathbf{w}_{i}=\left(\begin{array}{c}
\rho \\
\rho \mathbf{u} \\
\rho E
\end{array}\right),
$$

where $E=c_{v} T+\frac{1}{2} \mathbf{u}^{2}$ is the total energy with $c_{v}$ the specific heat at constant volume. The convective and viscous fluxes $\mathbf{F}_{i}^{c}$ and $\mathbf{F}_{i}^{v}$ are given by

$$
\mathbf{F}_{i}^{c}=\left(\begin{array}{c}
\rho \mathbf{u} \\
\rho \mathbf{u} \otimes \mathbf{u}+p \mathbb{I} \\
\rho E \mathbf{u}+p \mathbf{u}
\end{array}\right) \quad \text { and } \quad \mathbf{F}_{i}^{v}=\left(\begin{array}{c}
0 \\
2 \mu \mathbb{T} \\
\mathbf{u} 2 \mu \mathbb{T}+\kappa \nabla T
\end{array}\right)
$$

where $\mathbb{I} \in \mathbb{R}^{3}$ is the identity matrix, $\mu$ is the molecular viscosity, $\kappa$ the thermal conductivity and $\mathbb{T}$ is the strain rate tensor

$$
\mathbb{T}=\mathbb{S}-\frac{1}{3} \nabla \mathbf{u} \mathbb{I} \quad \text { with } \quad \mathbb{S}=\frac{1}{2}\left(\nabla \mathbf{u}+(\nabla \mathbf{u})^{T}\right) .
$$

Using the notation for the residual $\mathcal{R}\left(\mathbf{W}_{i}\right)$

$$
\mathcal{R}\left(\mathbf{W}_{i}\right)=\frac{1}{\operatorname{vol}\left(V_{i}\right)} \int_{\partial V_{i}} \mathbf{F}_{i}^{c} \cdot \mathbf{n} d S-\frac{1}{\operatorname{vol}\left(V_{i}\right)} \int_{\partial V_{i}} \mathbf{F}_{i}^{v} \cdot \mathbf{n} d S,
$$

where $\mathbf{n}$ is the normal vector of the boundary $\partial V_{i}$ of the control volume $V_{i}$, the NSE is given by

$$
\frac{\partial}{\partial t} \mathbf{W}_{i}+\mathcal{R}\left(\mathbf{W}_{i}\right)=0 \quad \text { in } V_{i} \quad \forall V_{i} \subset \Omega,
$$

with boundary conditions on $\Gamma=\partial \Omega$. Here we confine ourselves to farfield boundaries $\Gamma_{\text {farfield }}$ and optionally a viscous wall $\Gamma_{\mathrm{w}}$.

International Journal for Numerical Methods in Fluids, 70(12) pp. 1558-1572, 2012 
To account for turbulence, several turbulence models ranging from one-equation to Reynolds-stress-models are implemented. In the presented examples the $k-\omega$ linearized explicit algebraic stress (LEA) model [12] and the Menter-SST model [13] are employed. Regarding the numerical scheme, the spatial derivatives are approximated with a second order central difference scheme with artificial dissipation. The artificial dissipation stabilizes the scheme and can be either of scalar [14] or matrix type [15]. The influence of the two different schemes is examined in the first example in section 4.1. For the propagation in time a backward differentiation formula of second order is given by:

$$
\frac{3}{2 \Delta t} \mathbf{W}_{i}^{n+1}-\frac{4}{2 \Delta t} \mathbf{W}_{i}^{n}+\frac{1}{2 \Delta t} \mathbf{W}_{i}^{n-1}+\mathcal{R}\left(\mathbf{W}_{i}^{n+1}\right)=0 .
$$

Here $\mathbf{W}_{i}^{n+1}$ denotes the solution at the current physical time step $t_{n+1}$, the two previous steps are denoted by $\mathbf{W}_{i}^{n}$ and $\mathbf{W}_{i}^{n-1}$. This nonlinear steady-state problem is solved at each time step using the following dual-time stepping scheme in pseudo time $t^{*}$ :

$$
\frac{\partial}{\partial t^{*}} \mathbf{W}_{\mathbf{i}}^{\mathbf{n}+\mathbf{1}}+\mathcal{R}^{D T S}\left(\mathbf{W}_{i}^{n+1}\right)=0,
$$

with

$$
\mathcal{R}^{D T S}\left(\mathbf{W}_{i}^{\nu}\right)=\mathcal{R}\left(\mathbf{W}_{i}^{\nu}\right)+\frac{3}{2 \Delta t} \mathbf{W}_{i}^{\nu}-\frac{4}{2 \Delta t} \mathbf{W}_{i}^{n}+\frac{1}{2 \Delta t} \mathbf{W}_{i}^{n-1} .
$$

One implemented iterative solver is the explicit low-storage k-stage Runge-Kutta scheme [14]:

$$
\begin{aligned}
\mathbf{W}_{i}^{(0)} & =\mathbf{W}_{i}^{\nu} \\
\mathbf{W}_{i}^{(1)} & =\mathbf{W}_{i}^{(0)}-\alpha_{1} \Delta t^{*} \mathcal{R}\left(\mathbf{W}_{i}^{(0)}\right) \\
\vdots & \\
\mathbf{W}_{i}^{(k)} & =\mathbf{W}_{i}^{(0)}-\alpha_{k} \Delta t^{*} \mathcal{R}\left(\mathbf{W}_{i}^{(k-1)}\right) \\
\mathbf{W}_{i}^{\nu+1} & =\mathbf{W}_{i}^{(k)}
\end{aligned}
$$

where $\alpha_{1}, \ldots, \alpha_{k}$ are the Runge-Kutta coefficients. Several cycles of this scheme are executed to converge the solution $\mathbf{W}_{i}^{\nu}$ to a steady state in pseudo time. The time step $\Delta t^{*}$ is determined by the CFL-number. The simulation can be accelerated using residual smoothing, multi-grid technique and pre-conditioning [16].

\section{Chimera technique in the DLR TAU-code}

Details of the implementation of the Chimera technique in the DLR TAU-code can be found in [17]. A simulation with Chimera can be interpreted as a domain decomposition problem with overlap. For the sake of simplicity of the presentation, we introduce one background grid $\Omega_{\mathrm{bg}}$ that covers the whole computational domain $\Omega$ and include only one Chimera block $\Omega_{\mathrm{cg}}$, where $\bar{\Omega}_{\mathrm{cg}} \subset \Omega_{\mathrm{bg}}$. Denote $\Omega_{\text {hole }}$ with boundary $\Gamma_{\text {hole }}$ the so called hole-definition grid on a null-homotopic domain (loosely speaking a domain without holes) with $\bar{\Omega}_{\text {hole }} \subset \Omega_{\mathrm{cg}}$, that is associated with $\Omega_{\mathrm{cg}}$. Denote $\mathbf{W}^{\mathrm{bg}}$ the solution in $\Omega_{\mathrm{bg}} \backslash \bar{\Omega}_{\mathrm{hole}}$ and $\mathbf{W}^{\mathrm{cg}}$ the solution in $\Omega_{\mathrm{cg}}$. Moreover denote

International Journal for Numerical Methods in Fluids, 70(12) pp. 1558-1572, 2012 
$\Omega_{\mathrm{bg} \leftarrow \text { cg,ip }} \subset \Omega_{\mathrm{bg}}$ an interpolation region from $\Omega_{\mathrm{cg}}$ onto $\Omega_{\mathrm{bg}}$, which is located adjacent to $\Gamma_{\text {hole }}$. Similarly, denote $\Omega_{\mathrm{cg} \leftarrow \mathrm{bg}, \mathrm{ip}} \subset \Omega_{\mathrm{cg}}$ an interpolation region from $\Omega_{\mathrm{bg}}$ onto $\Omega_{\mathrm{cg}}$, located adjacent to $\Gamma_{\mathrm{cg}}=\partial \Omega_{\mathrm{cg}}$. The situation is shown in figure 1 .

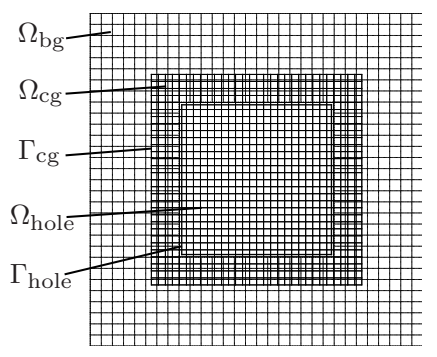

(a)

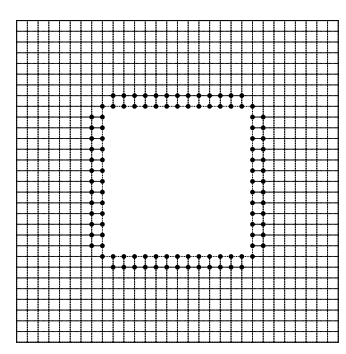

(b)

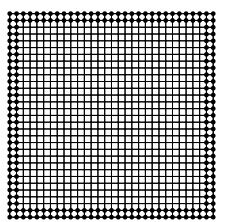

(c)

Fig. 1: (a) Background grid $\Omega_{\mathrm{bg}}$ with Chimera grid $\Omega_{\mathrm{cg}}$ and hole definition grid $\Omega_{\text {hole }}$ outlined by thick lines; (b) $\Omega_{\mathrm{bg}}$ : points in $\bar{\Omega}_{\text {hole }}$ are blanked out, points in $\Omega_{\mathrm{bg} \leftarrow \mathrm{cg} \text {,ip }}$ are marked; (c) $\Omega_{\mathrm{cg}}$ : points in $\Omega_{\mathrm{cg} \leftarrow \mathrm{bg}, \mathrm{ip}}$ are marked

Then we seek the solution $\mathbf{W}_{i}^{\mathrm{bg}}$ such that

$$
\frac{\partial \mathbf{W}_{i}^{\mathrm{bg}}}{\partial t}-\mathcal{R}\left(\mathbf{W}_{i}^{\mathrm{bg}}\right)=0 \text { in } V_{i} \quad \forall V_{i} \subset \Omega_{\mathrm{bg}} \backslash \bar{\Omega}_{\text {hole }}
$$

with the original boundary conditions on $\Gamma_{\mathrm{bg}} \backslash \bar{\Omega}_{\mathrm{hole}}$ and $\mathbf{W}^{\mathrm{bg}}=\mathbf{W}^{\mathrm{cg}}$ in $\Omega_{\mathrm{bg} \leftarrow \mathrm{cg} \text {,ip }}$ by interpolation.

Moreover we seek the solution on the Chimera grid $\mathbf{W}_{i}^{\mathrm{cg}}$ such that

$$
\frac{\partial \mathbf{W}_{i}^{\mathrm{cg}}}{\partial t}-\mathcal{R}\left(\mathbf{W}_{i}^{\mathrm{cg}}\right)=0 \quad \forall V_{i} \subset \Omega_{\mathrm{cg}}
$$

with $\mathbf{W}^{\mathrm{cg}}=\mathbf{W}^{\mathrm{bg}}$ in $\Omega_{\mathrm{cg} \leftarrow \mathrm{bg}, \text { ip }}$ by interpolation.

The interpolation domains $\Omega_{\mathrm{bg} \leftarrow \mathrm{cg}, \text { ip }}$ and $\Omega_{\mathrm{cg} \leftarrow \mathrm{bg} \text {,ip }}$ consist each of two layers of grid points, as each interpolation domain is built up of the set of grid points for which the spatial discretization operator would assess a node not belonging to $\Omega_{\mathrm{bg}} \backslash \bar{\Omega}_{\text {hole }}$ resp. to $\Omega_{\mathrm{cg}}$. The values of all conservative variables are interpolated using a trilinear interpolation (in a 3D computation). Since the overlap region does not decrease with mesh refinement trilinear interpolation is sufficient for maintaining the second order accuracy of the scheme in space according to [18]. This approach is in accordance to $\mathrm{Wu}$ [19], who showed that the interpolation of the numerical fluxes instead of the conservative variables may lead to a nonunique steady-state solution of problem (7) and (8).

For all dual cells an array $I B L A N K\left[V_{i}\right]$ is stored, with

$$
I B L A N K\left[V_{i}\right]= \begin{cases}0 & \text { if } V_{i} \in \Omega_{\mathrm{bg}} \text { and } V_{i} \subset \bar{\Omega}_{\text {hole }} \\ 1 & \text { else }\end{cases}
$$

The Runge-Kutta scheme in equation (6) is modified:

$$
\mathbf{W}_{i}^{(j)}=\mathbf{W}_{i}^{(0)}-I B L A N K\left[V_{i}\right] \alpha_{j} \Delta t^{*} \mathcal{R}\left(\mathbf{W}_{i}^{(j-1)}\right) \text { for } j=1, \cdots, k,
$$

International Journal for Numerical Methods in Fluids, 70(12) pp. 1558-1572, 2012 
ensuring that in the blanked points no update of the solution is computed. Instead an update of the conservative variables in the interpolation regions is performed by interpolation in each step.

$\Omega_{\mathrm{cg}}$ can be in motion relative to $\Omega_{\mathrm{bg}}$. The TAU-code allows one to prescribe translatory, rotational and composed trajectories, that are described as time dependent Fourier or polynomial series. In this case $\Omega_{\text {hole }}$ remains attached to $\Omega_{\mathrm{cg}}$. This requires an update of the interpolation regions $\Omega_{\mathrm{bg} \leftarrow \mathrm{cg} \text {,ip }}$ and $\Omega_{\mathrm{cg} \leftarrow \mathrm{bg} \text {,ip }}$ in each physical time step. A fast way to find the respective donor cells for the interpolation is the alternating digital tree [20], that reduces the complexity of the search to $\log _{2} \mathrm{~N}$ operations. The search result is a cartesian box which comprises the possible donor cells. For each cell an enveloping element with planar faces is constructed and it is checked if the receiving point $\mathbf{x}_{r}$ is located in the interior. If so, the following nonlinear system of equations is solved using a Newton-method:

$$
\sum_{i} f_{i}(\xi, \eta, \mu) \mathbf{x}_{i}=\mathbf{x}_{r}
$$

where $\mathbf{x}_{i}$ are the corners of the element, $\xi, \eta$ and $\mu$ are the local coordinates in the reference finite element and the $f_{i}$ are the weights of the linear, bilinear or trilinear ansatz functions for the respective element type with following property:

$$
f_{i}\left(\mathbf{x}_{j}\right)= \begin{cases}1 & \text { if } i=j \\ 0 & \text { if } i \neq j\end{cases}
$$

The $f_{i}$ take values $0 \leq f_{i}\left(\mathbf{x}_{r}\right) \leq 1$ if the point $\mathbf{x}_{r}$ lies inside the element and they are the interpolation weights of the trilinear interpolation. For a valid donor cell an additional requirement is that none of the corners is blanked. For this reason the overlap region must be sufficiently large. If more than two grids overlap, the search starts at the first block and proceeds until an appropriate donor cell is found. So if the background grid is the first block in the common grid, the solution is interpolated from there, even if its resolution is very coarse and thus afflicted with a higher discretization error. This must be payed attention for when the user chooses the sequence of the Chimera blocks. For a second order time discretization, like in equation (5), the solutions of the two previous time steps are required. Due to a moving block it can happen that a point in $\Omega_{\mathrm{bg}} \backslash \bar{\Omega}_{\text {hole }}$ that was blanked in one of the two previous time steps becomes active. In this case the values of the old time steps are reconstructed by interpolation of the respective values on $\Omega_{\mathrm{cg}}$. Since a different movement can be assigned to each Chimera block, vectorial values like coordinates and velocities must be suitably transformed.

The functionality of the TAU-code can be controlled via Python scripts. These enable to couple different types of motion and thus to automate the process of the grid movement from the viewpoint of the technical CFD-code usage. The type of motion and the duration of the single steps still must be defined by the user in advance.

The TAU-code can be used in parallel mode. For this purpose the grid is split into smaller sub-grids and each is treated by one processor. The values at the sub grid boundaries are communicated to the other domains. One important feature of this domain decomposition is a balanced distribution of work. With the Chimera approach it is not a priori known how much points are active on each domain due to the blanking of points. This may lead to a certain imbalance. Details of the efficiency

International Journal for Numerical Methods in Fluids, 70(12) pp. 1558-1572, 2012 
of the Chimera method in the DLR TAU-code can be found in [21]. If multi-grid is used to accelerate the convergence, the holes must also be defined on the coarse grids: All coarse grid cells that contain at least one blanked cell are marked as blanked. The solution is interpolated from the finer grid level to the coarser grid level to get values for the receiving points.

\section{$4 \quad$ Numerical test cases}

Several analytical vortex models like the potential vortex, the Rankine vortex, the Lamb-Oseen vortex and others can be found in literature, which are all of infinite size. Therefore a new finite vortex model is introduced, that consists of four spatial zones, separated by the radii $0<r_{c}<r_{m}<r_{o}$, and used within the numerical examples. Zone 1 and 2 are of Rankine-type, whereas the idea of damping $v_{\phi}$ by a function $\beta$ in zone 3 is taken from the Lamb-Oseen vortex. At $r_{o}$ the velocity $v_{\phi}$ is sufficiently small to be cut off, thus ensuring the finite size. The tangential velocity is defined as:

$$
v_{\phi}(r)=\left\{\begin{array}{ll}
\frac{\Gamma_{0} \cdot r}{2 \cdot \pi \cdot r_{c}^{2}} & \text { if } r<r_{c} \\
\frac{\Gamma_{0}}{2 \cdot \pi \cdot r} & \text { if } r_{c} \leq r<r_{m} \\
\frac{\Gamma_{0}}{2 \cdot \pi \cdot r} \cdot \beta & \text { if } r_{m} \leq r<r_{o} \\
0 & \text { if } r_{o}<r
\end{array}, \quad \text { with } \quad \beta=e^{\frac{\left(r-r_{m}\right)^{2}}{\delta}} .\right.
$$

In the following examples a molecular viscosity of $\mu=1.72 \times 10^{-5} \mathrm{~kg} /(\mathrm{ms})$ and a density of $\rho=1.294 \mathrm{~kg} / \mathrm{m}^{3}$ are used. To take into account the effects of turbulence, in (2) the molecular viscosity is replaced by the effective viscosity $\mu_{\mathrm{eff}}=\mu+\mu_{\mathrm{t}}$. The so called turbulent viscosity $\mu_{\mathrm{t}}$ is computed using the LEA-turbulence model in 4.1-4.4 and the Menter-SST turbulence model in example 4.5.

\subsection{Preservation of a finite vortex in time}

The aim of this section is to specify the settings for the numerical scheme and the time and grid resolution required for a single vortex on a fine mesh which will become the Chimera grid in the later test cases. It will become clear in section 4.2 that we are interested in the situation, that the vortex is at rest relatively to the (Chimera) grid, hence $v_{\infty}=0 \frac{\mathrm{m}}{\mathrm{s}}$. The vortex, as defined in (12), is described by $r_{c}=2 \mathrm{~m}, r_{m}=4 \mathrm{~m}$, $r_{o}=10 \mathrm{~m}$ and $\Gamma_{0}=60$ and $\delta=10 \mathrm{~m}^{2}$. The computational domain is chosen as $100 \mathrm{~m} \times 100 \mathrm{~m}$. Three different structured grids are used: a coarse grid consisting of $101 \times 101$ points, a fine grid with $201 \times 201$ points and a very fine grid with $301 \times 301$ points. The points are clustered in the center of the grid, where the origin of the vortex is located. For example in the fine grid $100 \times 100$ points reside in the area of $(-10 \mathrm{~m}, 10 \mathrm{~m}) \times(-10 \mathrm{~m}, 10 \mathrm{~m})$, which is covered by the outer radius $r_{o}$. The physical time step sizes of $\Delta t=10^{-1} \mathrm{~s}, \Delta t=10^{-2} \mathrm{~s}, \Delta t=10^{-3} \mathrm{~s}$ and $\Delta t=10^{-4} \mathrm{~s}$ are tested. The viscous fluxes are discretized with a second order central scheme with artificial dissipation, on the one hand with a scalar formulation [14] and on the other hand with a matrix formulation [15]. The calculations are performed with and without pre-conditioning[16].

Figure 2 illustrates the development of the tangential velocity on the coarse and fine grid with scalar and matrix dissipation schemes. Here the time step size is chosen as

International Journal for Numerical Methods in Fluids, 70(12) pp. 1558-1572, 2012 


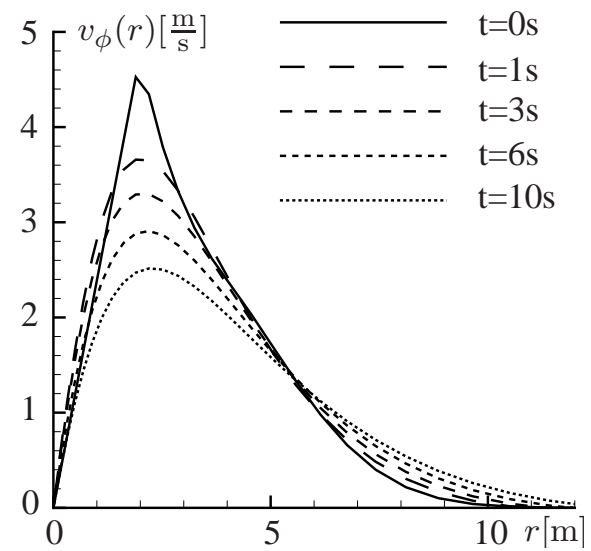

(a)

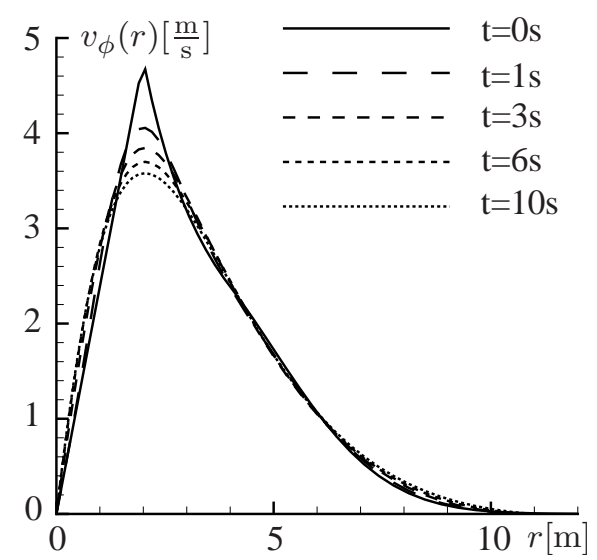

(c)

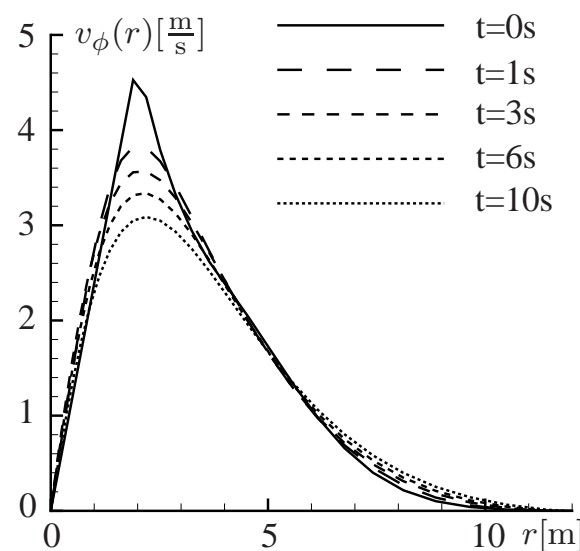

(b)

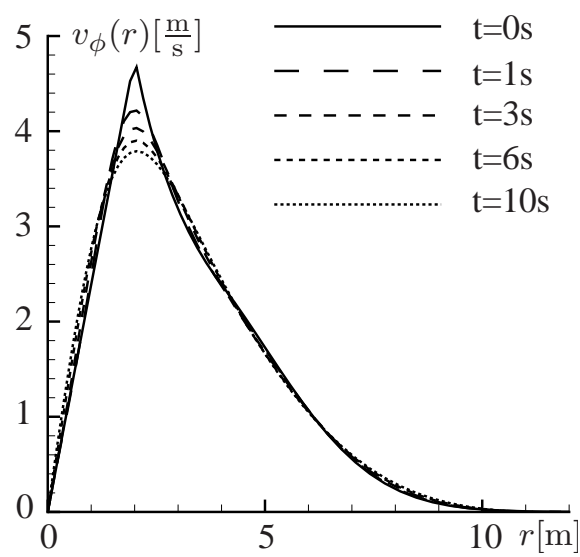

(d)

Fig. 2: Development of tangential velocity $v_{\phi}$, using $\Delta t=10^{-2}$ s and pre-conditioning: (a) $101 \times 101$ points and scalar dissipation; (b) $101 \times 101$ points and matrix dissipation; (c) $201 \times 201$ points and scalar dissipation; (d) $201 \times 201$ points and matrix dissipation

$\Delta t=10^{-2}$ s and pre-conditioning is used. The numerical dissipation on the coarse grid is too high to preserve the vortex in time. Independent of the chosen artificial dissipation, the velocity decays discernibly during the simulation time. On the fine grid the vortex is preserved much better, with matrix dissipation only the unphysical peak at $r_{c}$ is damped. Further simulations on the very fine grid did not improve the results significantly, hence the resolution of the fine grid is taken as sufficient.

Regarding the study of the time step size, the simulation became unstable and crashed after $5 \mathrm{~s}$ of simulation time with the largest time step of $\Delta t=10^{-1} \mathrm{~s}$. The differences between the other three time steps were marginally, such that a time step of $\Delta t=10^{-2} \mathrm{~s}$ is used. As the results show, matrix dissipation helps reducing the dissipation and the vortex is better preserved. The results without pre-conditioning show much higher dissipation, such that pre-conditioning is considered crucial and is used in the following examples.

International Journal for Numerical Methods in Fluids, 70(12) pp. 1558-1572, 2012 


\subsection{Transport of a finite vortex}

Now that the optimal settings on the Chimera grid are found, the influence of the Chimera technique and the exchange of boundary data with the much coarser background grid is studied. For this purpose a background grid on a rectangular computational domain of $(-50 \mathrm{~m}, 150 \mathrm{~m}) \times(-50 \mathrm{~m}, 50 \mathrm{~m})$ is defined. Two different resolutions are compared for the background grid. The fine grid contains $601 \times 201$ equidistant points, the coarse grid contains $101 \times 51$ points. In both calculations the Chimera block containing the vortex covers a computational domain of $25 \mathrm{~m} \times 25 \mathrm{~m}$. It has $201 \times 201$ points, with $130 \times 130$ points clustered in the area of $(-10 \mathrm{~m}, 10 \mathrm{~m}) \times(-10 \mathrm{~m}, 10 \mathrm{~m})$. The hole definition grid is of size $23 \mathrm{~m} \times 23 \mathrm{~m}$ for the fine background grid and $18 \mathrm{~m} \times 18 \mathrm{~m}$ for the coarse background grid. These different hole definitions guarantee a sufficiently large overlap region. While with the fine background grid the size of the elements in the overlap region resembles that in the vortex grid, this is not the case for the coarse background grid. The vortex is again defined by $r_{c}=2 \mathrm{~m}, r_{m}=4 \mathrm{~m}, r_{o}=10 \mathrm{~m}$ and $\Gamma_{0}=60$ and $\delta=10 \mathrm{~m}^{2}$ and is located at the center of the vortex Chimera block. The outer velocity is prescribed as $v_{\infty, x}=100 \frac{\mathrm{m}}{\mathrm{s}}$ and the same velocity is used for the movement of the vortex grid.

In figure 3 the initial setup and the position of the Chimera block after $2 \mathrm{~s}$ of simulation time are shown. In figure 4 the results of both tested settings can be seen. There is no noticeable difference between them, showing that the coarse background grid does not have noticeable dissipative effects on the vortex on the Chimera grid.

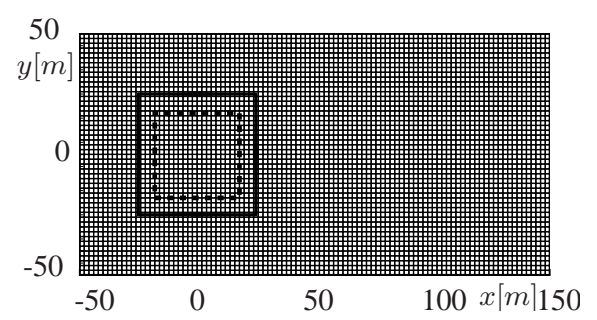

(a)

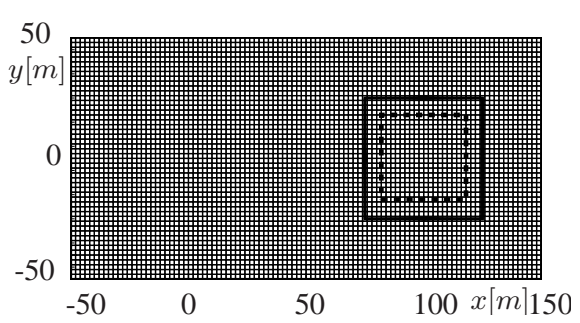

(b)

Fig. 3: Position of the vortex transport block, outlined by thick line, and hole definition grid, outlined by thick dashed line: (a) at simulation start and (b) after $2 \mathrm{~s}$ of simulation time

\subsection{Interaction of a finite vortex with a NACA0012 airfoil}

The interaction of a finite vortex with an airfoil is studied. The hybrid background grid is built around the contour of the NACA0012 airfoil and covers a circular computational domain with a radius of $100 \mathrm{~m}$. It consists of approximately 55000 points, with about 400 points on the surface and 27 structured layers. The chord length of the airfoil is $c=1 \mathrm{~m}$ and the angle of attack is $\alpha=0^{\circ}$. The Reynolds number is $R e=3.8 \times 10^{6}$, the Mach number is $M a=0.15$ and thus the free stream velocity is $v_{\infty, x}=50 \frac{\mathrm{m}}{\mathrm{s}}$. The same velocity is chosen for the movement of the structured vortex transport Chimera block. This block covers a computational domain of $30 \mathrm{~m} \times 30 \mathrm{~m}$ and is discretized with $151 \times 151$ equidistant points. The equidistant spacing is chosen because during the simulation the Chimera block will be halted and the vortex

International Journal for Numerical Methods in Fluids, 70(12) pp. 1558-1572, 2012 


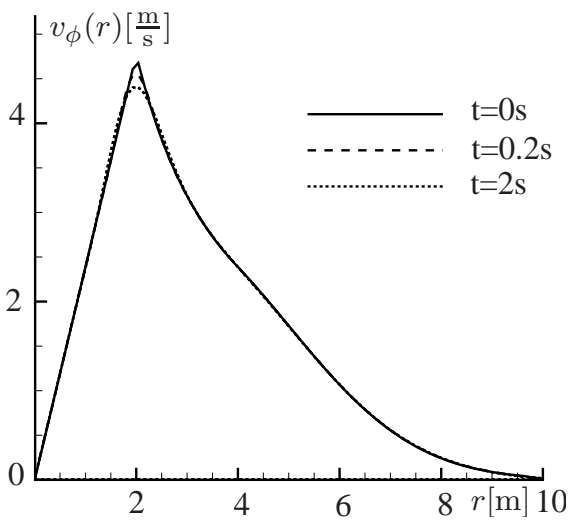

(a)

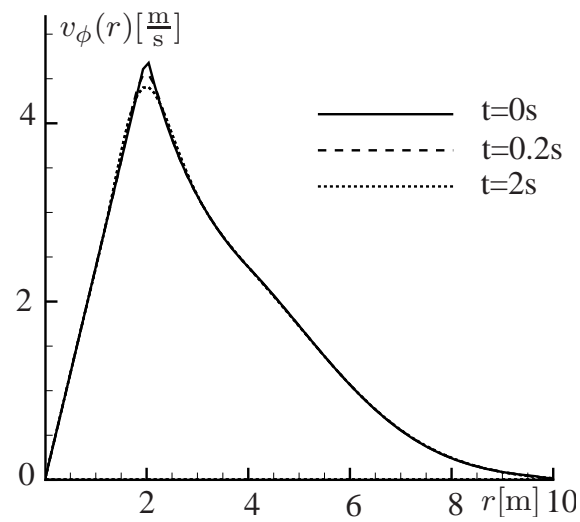

(b)

Fig. 4: Time evolution of the local tangential velocity of a finite vortex transported on a fine Chimera mesh: (a) with a fine and (b) with a coarse background grid, see also figure 3

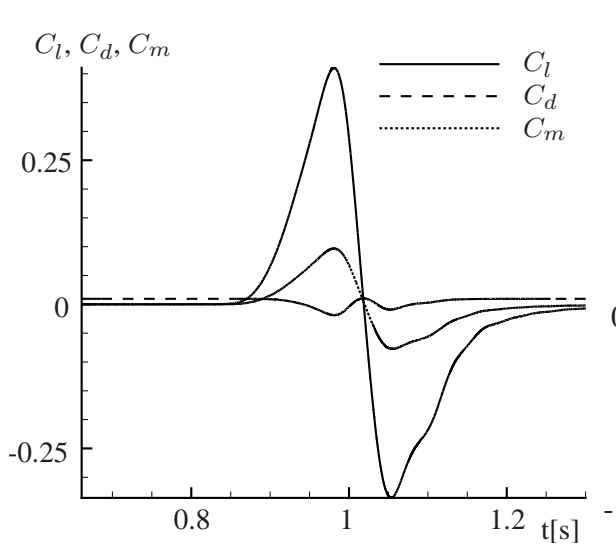

(a)

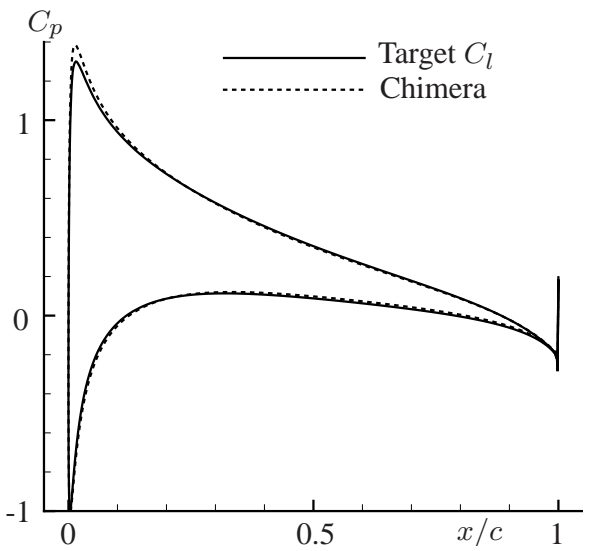

(b)

Fig. 5: (a) Curve of lift, drag and moment coefficient during the interaction of a finite analytical vortex with a NACA0012 airfoil; (b) comparison of Chimera simulation at maximum lift and corresponding undisturbed simulation at the same target lift at $\alpha=3.7^{\circ}$

will travel through the Chimera grid onto the background grid and thus must be adequately resolved everywhere.

The Chimera block is initially located at $x=-50 \mathrm{~m}$ upstream of the airfoil, with respect to its center. This distance is sufficiently large to prevent an influence of the vortex on the flow around the airfoil. The vortex is defined by $r_{c}=1.5 \mathrm{~m}, r_{m}=3 \mathrm{~m}$, $r_{o}=7 \mathrm{~m}$ and $\Gamma_{0}=60$ and $\delta=5 \mathrm{~m}^{2}$ and is located at the center of the vortex Chimera block.

To initialize the simulation at $t_{0}=0 \mathrm{~s}$ a converged solution on the background grid without the Chimera block is computed. Now the Chimera block with the initial 

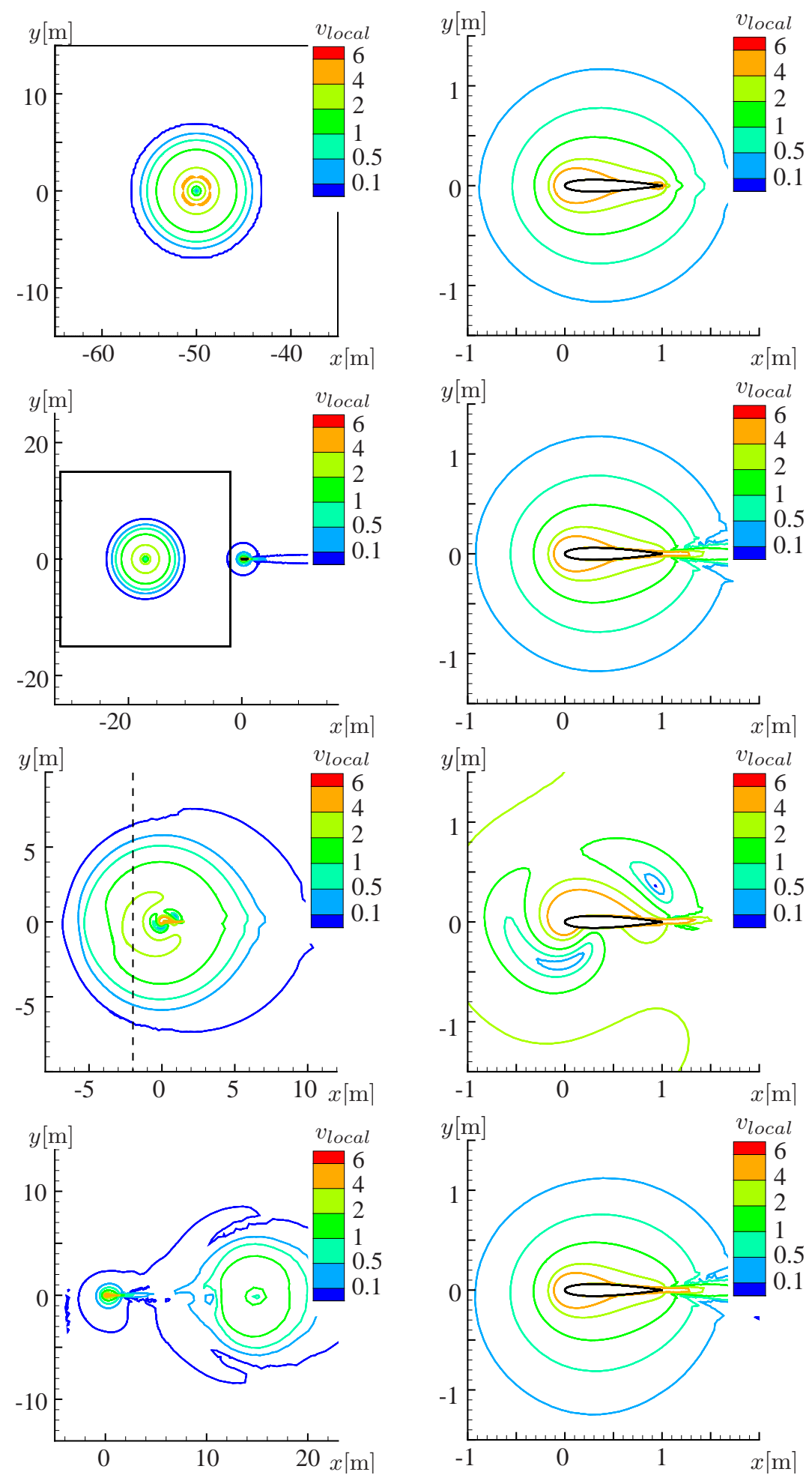

Fig. 6: Interaction of an analytical vortex with the NACA0012 airfoil at $\alpha=0^{\circ}$. Contours of velocity at different time steps: from top to bottom $t_{0}=0 \mathrm{~s}, t_{1}=0.66 \mathrm{~s}, t_{2}=1 \mathrm{~s}$ and $t_{3}=1.3 \mathrm{~s}$

International Journal for Numerical Methods in Fluids, 70(12) pp. 1558-1572, 2012 
values of the vortex is introduced into the background grid. Then the vortex grid is set into motion and the vortex is transported towards the airfoil. After $t_{1}=0.66 \mathrm{~s}$ the right interpolation boundary of the Chimera block has traveled to a distance of $2 \mathrm{~m}$ upstream of the airfoil. On the one hand the Chimera block must not intersect the airfoil, since inside the airfoil contour no adequate interpolation points are defined. On the other hand the background grid is very fine in the vicinity of the airfoil, such that the preservation of the vortex on the background grid can be assumed. Therefore at $t_{1}$ the movement of the Chimera block is stopped. The vortex passes the boundary of the Chimera block and is then transported on the background grid. At $t_{2}=1 \mathrm{~s}$ the center of the vortex has reached the center of the background grid. It has divided into two parts, of which one travels above the upper side of the airfoil and the other one below the lower side. At $t_{3}=1.3 \mathrm{~s}$ the vortex has passed the airfoil. At this time step the simulation is stopped, because the background grid is not fine enough to preserve the vortex any longer.

The influence of the interaction with the vortex on the integral coefficients is shown in figure 5 (left). This interaction acts like a change in the effective angle of attack. First the effective $\alpha$ is increased, which results in positive values of $C_{l}$ and $C_{m}$, then $\alpha$ effectively decreases, leading to negative values. The maximal value of $C_{l}=$ 0.41 is reached at $t_{m}=0.98 \mathrm{~s}$ with a corresponding moment coefficient of $C_{m}=$ 0.096. A reference simulation without vortex interaction with target lift $C_{l}=0.41$ is performed. It yields an angle of attack of $\alpha=3.7^{\circ}$ and a moment coefficient $C_{m}=$ 0.101 that is in good agreement. The comparison of the pressure distributions of the Chimera simulation and the reference undisturbed simulation is shown 5 (right). In figure 6 the contour lines of the velocity at different instances in time are shown.

\subsection{Interaction of a finite vortex with an ONERA-A airfoil near stall}

The interaction of a finite vortex with the ONERA-A airfoil near stall is investigated at $R e=2 \times 10^{6}$ and $M a=0.15$. The chord length of the airfoil is $c=1 \mathrm{~m}$ and the angle of attack is $\alpha=13.3^{\circ}$. At this angle of attack the airfoil is already close to stall at undisturbed onflow conditions. The subsequent interaction with a vortex is therefore expected to result in stall. An outer velocity of $v_{\infty, x}=51.5 \frac{\mathrm{m}}{\mathrm{s}}$ is prescribed and the time step is chosen as $\Delta t=2.5 \times 10^{-4} \mathrm{~s}$. The finite vortex is defined by the same properties as in the last example and the vortex Chimera block is also equidistant. The hybrid background grid is built around the airfoil and consists of about 39000 points, with 530 points on the surface and 33 points in the structured layers.

It was checked that placing the vortex into the flow field at a distance of $12 \mathrm{~m}$ from the airfoil did not change the flow at the airfoil. However this shortened distance reduces the computational effort, since the overall simulation time reduces to $0.42 \mathrm{~s}$.

The simulation is again initialized at $t_{0}=0 \mathrm{~s}$ with a converged solution on the background grid without disturbance and the Chimera vortex block is initially located at $x=-12 \mathrm{~m}$ upstream of the airfoil. At $t_{1}=0.16 \mathrm{~s}$ the movement of the Chimera block is stopped and the vortex passes the Chimera boundary and is further transported on the background grid. At $t_{2}=0.3 \mathrm{~s}$ the vortex has been mostly convected onto the background grid and its core has reached the airfoil where a strong interaction occurs. Finally it has traveled past the airfoil at $t_{3}=0.42 \mathrm{~s}$.

International Journal for Numerical Methods in Fluids, 70(12) pp. 1558-1572, 2012 


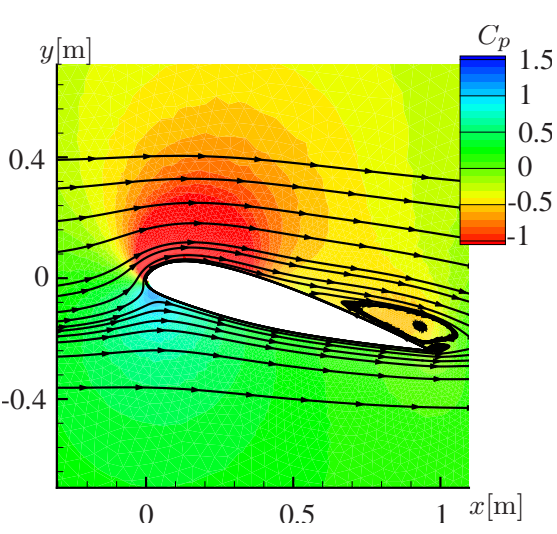

(a)

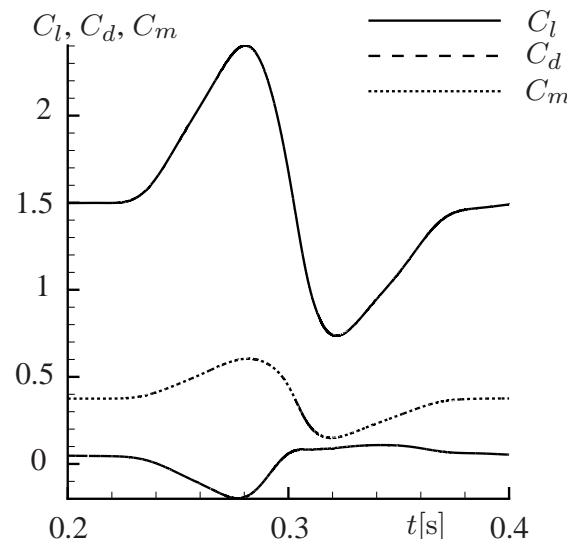

(b)

Fig. 7: (a) Pressure distribution and streamlines during the interaction of the vortex with the ONERA-A airfoil at $t_{2}=0.3 \mathrm{~s}$; (b) the curves of the integral coefficients

Figure 7 (left) shows the pressure distribution at the airfoil during the interaction with the vortex at $t_{2}=0.3 \mathrm{~s}$. Flow separation occurs at the trailing edge of the airfoil. The separation region is much larger than for the undisturbed onflow conditions. On the right side the time dependent behavior of the integral coefficients is shown. Again the interaction of the vortex with the airfoil acts like a change of the effective angle of attack. In figure 8 the contour levels of the velocity during the course of the simulation are shown. It can be seen, that the vortex is well preserved during the transport. The interaction with ONERA-A airfoil leads to a decrease of the strength of the vortex. Here it becomes apparent that also the airfoil has an effect on the vortex.

\subsection{Interaction of a vortex generated by a ramping airfoil with a two-element airfoil}

Now as a last example a realistic vortex is generated, transported and then interacts with a two-element airfoil at $R e=2 \times 10^{6}, M a=0.145$ and thus $v_{\infty, x}=50 \frac{\mathrm{m}}{\mathrm{s}}$. The geometry of the airfoil is very close to an industrial geometry by airbus and therefore is confined. The setup is typical for a gust generator in aerodynamic research facilities. It is embedded in a wind tunnel, whose dimensions are $6 \mathrm{~m} \times 1.3 \mathrm{~m}$. The tunnel is discretized with a coarse mesh of 12000 points. It is used as background grid $B G$ in which three Chimera blocks are contained. The initial setup is shown in figure 9.

The first block $C G_{1}$ is a hybrid grid with a diameter of $0.5 \mathrm{~m}$ around a NACA0012 airfoil with a chord length of $c=0.3 \mathrm{~m}$ at $\alpha_{0}=0^{\circ}$. The number of grid points is 33000 , with 280 surface points and 34 points in the structured layers. The second grid $C G_{2}$, an equidistant structured grid with $151 \times 151$ points, covers an area of $1 \mathrm{~m} \times 1 \mathrm{~m}$. The third block $C G_{3}$ is a hybrid grid with a diameter of $0.6 \mathrm{~m}$ around a two-element airfoil, the FNG ("Flügel neuer Generation", new generation airfoil). The retracted chord length is $c=0.6 \mathrm{~m}$, in this case the flap is deployed. The grid consists of about 100000 points, with 1000 surface points and 53 points in the structured layers.

International Journal for Numerical Methods in Fluids, 70(12) pp. 1558-1572, 2012 


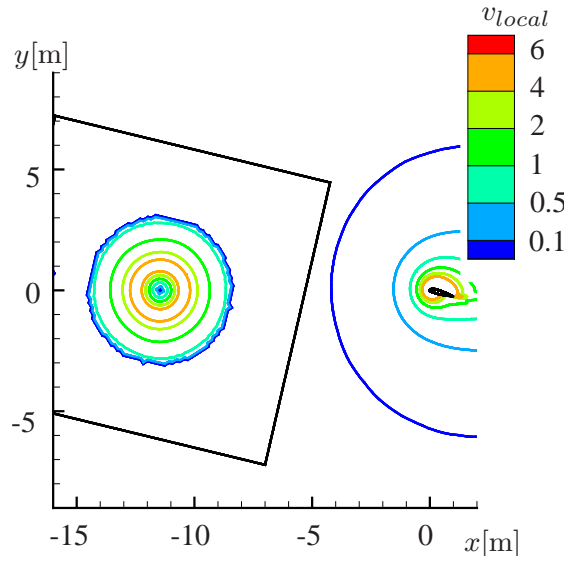

(a)

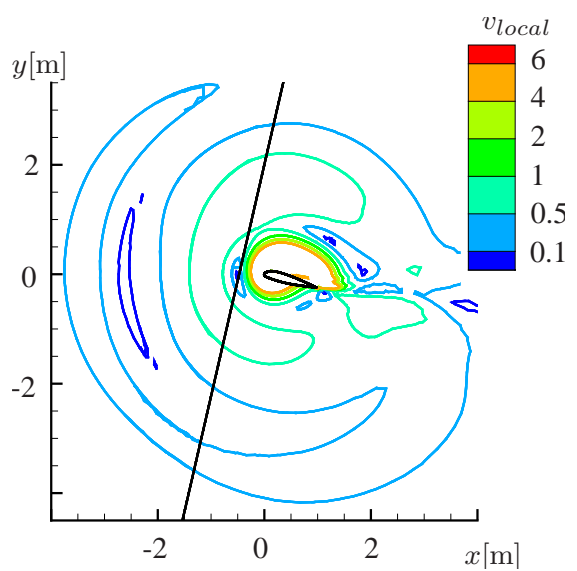

(c)

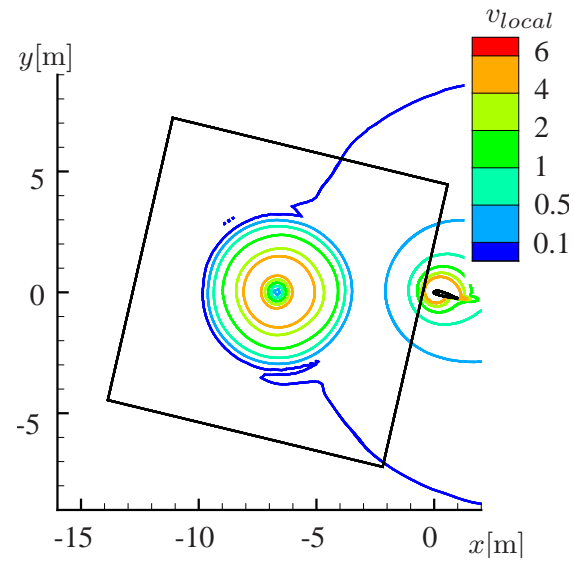

(b)

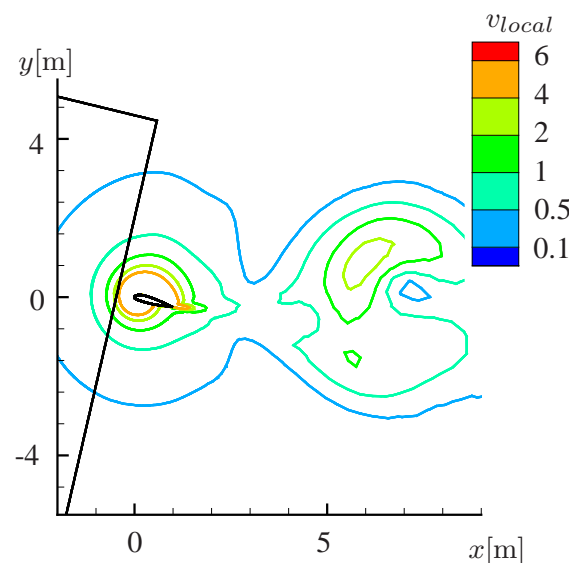

(d)

Fig. 8: Interaction of an analytical vortex with the ONERA-A airfoil at $\alpha=13.3^{\circ}$. Contours of velocity at $t_{0}=0 \mathrm{~s}(\mathrm{a}), t_{1}=0.16 \mathrm{~s}(\mathrm{~b}), t_{2}=0.3 \mathrm{~s}(\mathrm{c})$ and $t_{3}=0.42 \mathrm{~s}(\mathrm{~d})$

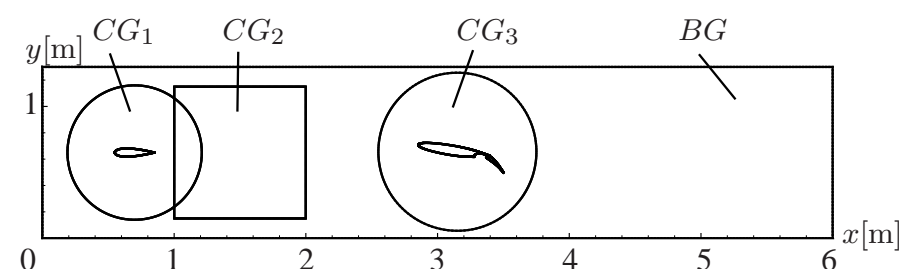

Fig. 9: Initial setup of the test case

This example demonstrates the advantage of the Chimera method: the second grid $C G_{2}$ is used to preserve the vortex while it travels towards the second airfoil. Without this grid it would be necessary to globally refined the wind tunnel grid $B G$. The cell size in the vortex transport grid $C G_{2}$ is four times smaller than in the background grid $B G$, therefore, the number of points in the background grid would increase by a factor of 16 if the same resolution was used. The number of additional points, and 
of computational resources respectively, would be even higher in a three dimensional calculation. This gain of performance dominates the additional cost in CPU-time that is introduced due to the Chimera interpolation by far.

A precursor unsteady RANS simulation including all Chimera blocks without movement of any block is performed until the integral lift coefficient is converged to a constant value of $C_{l}=2.03$. This solution is used to initialize the simulation at $t_{0}=0 \mathrm{~s}$. Then the generation of the vortex is started, by rotating the NACA0012 airfoil Chimera block $C G_{1}$ by $\alpha=10^{\circ}$ within $1.5 \times 10^{-2}$ s. During this pitching motion a realistic vortex is formed at the trailing edge. This vortex separates and is convected downstream. At $t_{1}=1.5 \times 10^{-2} \mathrm{~s}$ the rotation of the NACA0012 profile is stopped and its deflection angle stays at constant $\alpha=10^{\circ}$ for the rest of the simulation time. Now the vortex, that is convected with the free stream velocity, travels onto the second Chimera block $C G_{2}$ for the vortex transport. Once it is completely contained in $C G_{2}$, at $t_{2}=1.95 \times 10^{-2} \mathrm{~s}$, this block is set in motion with the same prescribed velocity as the free stream velocity. The vortex transport Chimera block $C G_{2}$ is moved downstream until it reaches the FNG airfoil and its Chimera block $C G_{3}$ at $t_{3}=3.3 \times 10^{-2} \mathrm{~s}$. Then the vortex transport block $C G_{2}$ is stopped and the vortex passes onto the FNG Chimera block $C G_{3}$. It travels further past the airfoil and the interaction of the vortex with the FNG airfoil takes place. At $t_{4}=4.44 \times 10^{-2} \mathrm{~s}$ the lift reaches its maximal value of $C_{l}=2.137$. Finally at $t_{5}=3.3 \times 10^{-2} \mathrm{~s}$ the vortex has passed the airfoil. The time-dependent behavior of the lift coefficient at the FNG

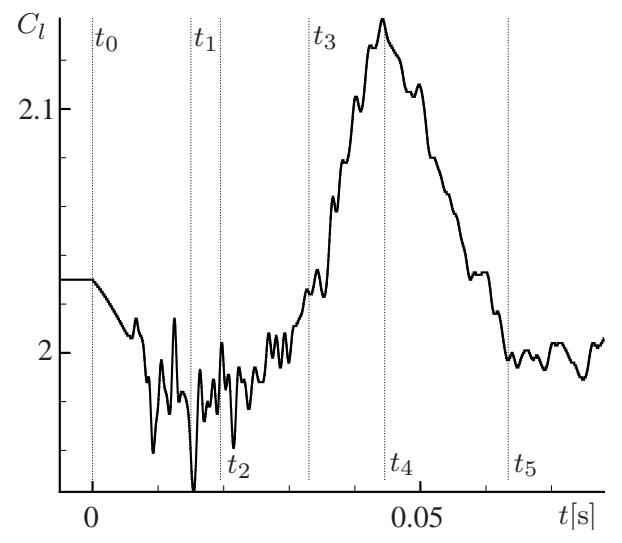

(a)

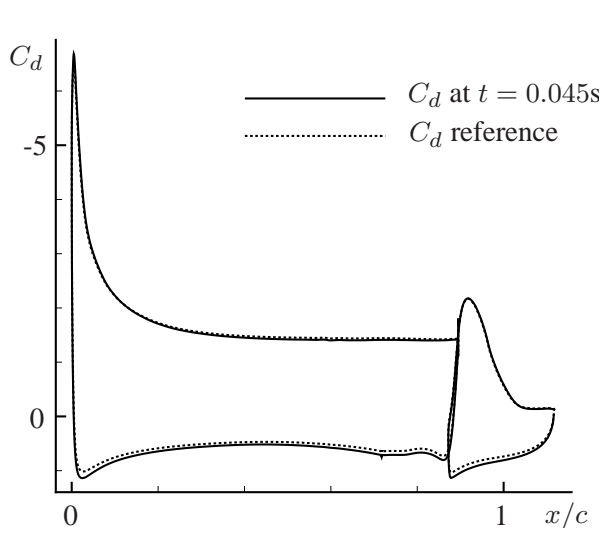

(b)

Fig. 10: (a) Curve of the lift coefficient during the simulation; (b) comparison of the pressure distribution at $t=0.045 \mathrm{~s}$ and at $\alpha=1.0$

airfoil is shown in figure 10 (left). Due to the increased blockage of the wind tunnel, the lift coefficient decreases during the rotation of the NACA0012 profile. As soon as the vortex reaches the FNG airfoil, the effect on $C_{l}$ is again the same as an increase of the effective angle of attack.

In a reference simulation of the FNG-airfoil with undisturbed onflow conditions without the NACA0012 and vortex transport Chimera blocks, the same lift of $C_{l}=2.137$ is reached at an angle of attack of $\alpha=1.0^{\circ}$. The comparison of the pressure distribution at the maximal lift at $t_{4}$ with the undisturbed simulation at $\alpha=1.0^{\circ}$ is shown in figure 10 (right). A plot of the vorticity at the described physical time steps is shown in figure 11.

International Journal for Numerical Methods in Fluids, 70(12) pp. 1558-1572, 2012 

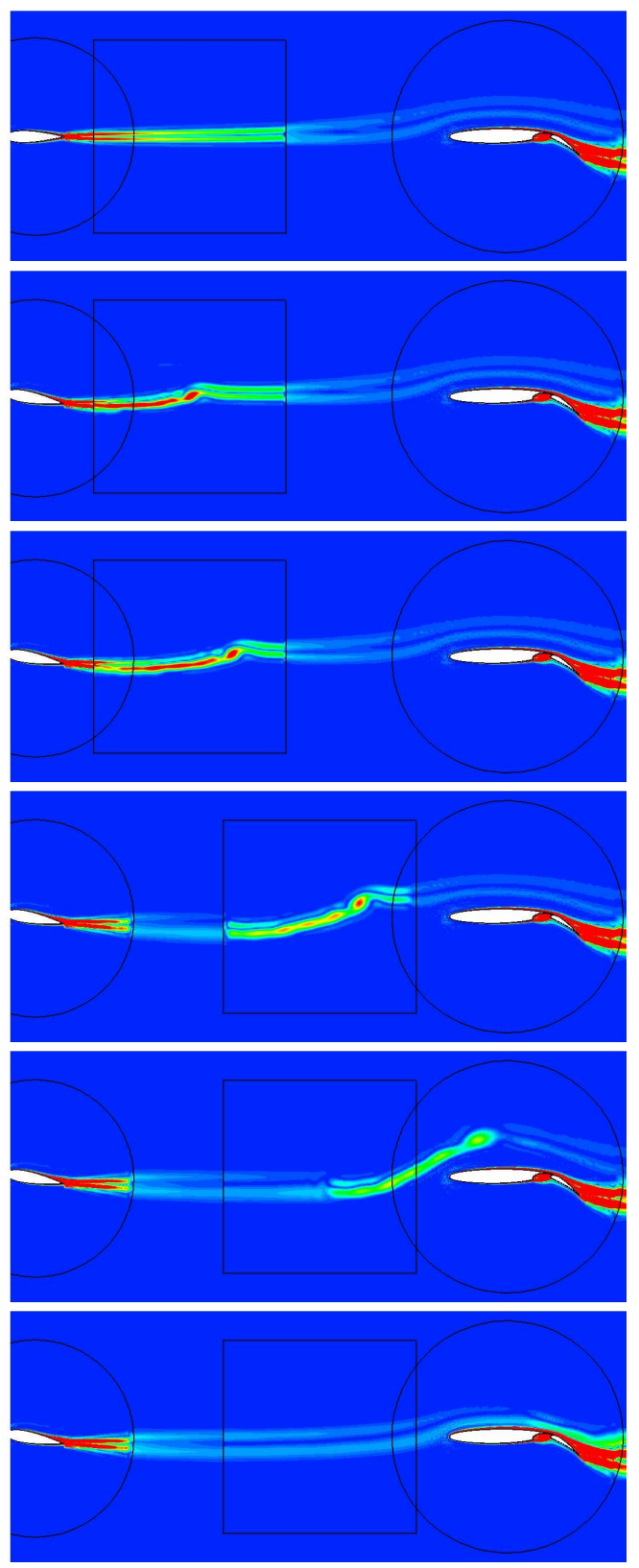

Fig. 11: Contour of vorticity during the generation, transport and interaction of a vortex with the FNG airfoil, from top to bottom: the initial flow field at $t_{0}=0 \mathrm{~s}$; after the rotation of the NACA0012 profile at $t_{1}=1.5 \times 10^{-2} \mathrm{~s}$; the vortex is completely convected onto the vortex transport grid at $t_{2}=1.95 \times 10^{-2} \mathrm{~s}$; the vortex transport grid reaches the FNG two-element airfoil at $t_{3}=3.3 \times 10^{-2} \mathrm{~s}$; the maximal lift is reached at $t_{4}=4.44 \times 10^{-2} \mathrm{~s}$ and the vortex has passed the FNG airfoil at $t_{5}=3.3 \times 10^{-2} \mathrm{~s}$.

International Journal for Numerical Methods in Fluids, 70(12) pp. 1558-1572, 2012 


\section{Conclusion}

In this paper we presented a Chimera based method for the simulation of the generation and transport of a vortex and its interaction with an airfoil. An analytical vortex of finite extent has been defined. The prerequisite numerical scheme as well as the spatial and temporal resolution for maintaining a vortex during the course of a simulation have been examined. It has been demonstrated, that the Chimera technique can be applied to transport a vortex on a fine grid block while using a coarse background grid without significantly altering it due to numerical dissipation. Then the Chimera approach has been used to simulate the interaction between an analytical vortex and an airfoil. Moreover, stall due to the interaction of a vortex with an airfoil at an angle of attack near stall has been predicted. Finally the Chimera technique was successfully used to generate a vortex by a ramping airfoil, to transport this vortex towards a two-element airfoil and to predict the interaction. Thus it is possible to reproduce the operating principle of a gust generator as it is often used in wind tunnels in aerodynamic research facilities.

At the present stage the user needs to specify the design of the vortex transport grid (i.e. the spacing and the initial position of the mesh), the movement of the mesh with the inflow velocity and a position sufficiently upstream of the airfoil where the mesh is finally stopped. From a perspective of industrial usage an automatization of these steps is desirable. For this a method to detect the vortex core and the proper resolution is needed, which is seen as a long term research goal.

Now, that the applicability of the Chimera technique to transport disturbances in the onflow conditions with a fine transport grid on a coarse background grid has been demonstrated, further applications will include more realistic disturbances. These will include atmospheric disturbances, as in [22], where a comparison study between synthetically generated turbulent wind fields and Large-Eddy Simulations (LES) is presented. Furthermore it is planned to apply a turbulence resolving model like LES or a hybrid RANS/LES approach to investigate the influence of different scales of the flow structures in the disturbance.

\section{Acknowledgment}

The authors gratefully acknowledge the support by the "Deutsche Forschungsgemeinschaft DFG" (German Research Foundation) that funded this research within the DFG FOR 1066 group. Moreover the valuable discussions with R. Radespiel, A. Probst, P. Scholz, D. Hahn (all Technical University Braunschweig) and T. Schwarz and R. Heinrich (both DLR Braunschweig) are gratefully acknowledged. Finally we are very grateful to the comments and suggestions by the reviewer which helped to significantly improve the manuscript.

\section{References}

[1] Coton FN, Marshall JS, Galbraith RAM, Green RB. Helicopter tail rotor orthogonal blade vortex interaction. Progress in Aerospace Sciences 2004; 40(7):453-

International Journal for Numerical Methods in Fluids, 70(12) pp. 1558-1572, 2012 
486, doi:10.1016/j.paerosci.2004.11.001.

[2] Leclercq D, Doolan C. The interaction of a bluff body with a vortex wake. Journal of Fluids and Structures 2009; 25(5):867-888, doi:10.1016/j.jfluidstructs. 2009.02.005.

[3] Abate M. Gust boundary condition for the DLR TAU-code. Technical Report IB 124-2007/9, DLR, Braunschweig, Germany 2007.

[4] Svärd M, Lundberg J, Nordström J. A computational study of vortex-airfoil interaction using high-order finite difference methods. Computers and Fluids 2010; 39(8):1267-1274.

[5] Alrutz T, Orlt M. Parallel dynamic grid refinement for industrial applications. ECCOMAS CFD 2006, Wesseling, Oñate, Périaux (eds.), TU Delft, 2006.

[6] Volkov EA. A finite difference method for finite and infinite regions with piecewise smooth boundaries. Doklady 1966; 168(5):744-747.

[7] Volkov EA. The method of composite meshes for finite and infinite regions with piecewise smooth boundaries. Proc. Steklov Inst. Math. 1968; 96:145-185.

[8] Starius G. On composite mesh difference methods for hyperbolic differential equations. Numer. Math. 1980; 35:241-255.

[9] Benek J, Dougherty F. A flexible grid embedding technique with application to the Euler equations. AIAA-83-1944, 1983.

[10] Benek J, Buning P, Steger G. A 3-D chimera grid embedding technique with application to the Euler equations. AIAA-1985-1523, 1985.

[11] Schwamborn D, Gerhold T, Heinrich R. The DLR Tau-Code: Recent Applications in Research and Industry. ECCOMAS CFD 2006, Wesseling, Oñate, Périaux (eds.), TU Delft, 2006.

[12] Rung T, Lübcke H, Franke M, Xue L, Thiele F, Fu S. Assessment of Explicit Algebraic Stress Models in Transonic Flows. Proc. of 4 th Symp. on Engineering Turbulence Modelling and Measurements, France, Elsevier: Amsterdam, 1999; 659-668, doi:doi:10.1016/B978-008043328-8/50063-1.

[13] Menter F. Zonal two-equation $\mathrm{k}-\omega$ turbulence model for aerodynamic flows. AIAA-93-2906, 1993.

[14] Jameson A, Schmidt W, Turkel E. Numerical solution of the Euler equations by finite volume methods using Runge-Kutta time-stepping schemes. AIAA-19811259, 1981.

[15] Swanson RC, Radespiel R, Turkel E. On some numerical dissipation schemes. J. Comput. Phys. 1998; 147:518-544, doi:http://dx.doi.org/10.1006/jcph.1998. 6100 .

[16] Birken P, Meister A. Stability of preconditioned finite volume schemes at low mach numbers. BIT Numerical Mathematics 2005; 45:463-480. 10.1007/s10543005-0009-0.

International Journal for Numerical Methods in Fluids, 70(12) pp. 1558-1572, 2012 
[17] Schwarz T. Ein blockstrukturiertes Verfahren zur Simulation der Umströmung komplexer Konfigurationen. Technical Report DLR-FB 2005-20, DLR, Braunschweig, Germany 2005.

[18] Chesshire G, Henshaw WD. Composite overlapping meshes for the solution of partial differential equations. Journal of computational physics 1990; 90:1-64, doi:10.1016/0021-9991(90)90196-8.

[19] Wu ZN. Uniqueness of steady-state solutions for difference equations on overlapping grids. SIAM J. Numer. Anal. 1996; 33:1336-1357, doi:10.1137/ S003614299325609X.

[20] Bonet J, Peraire J. An alternating digital tree (ADT) algorithm for 3D geometric searching and intersection problems. International Journal for numerical methods in engineering 1991; 31:1-17.

[21] Madrane A, Raichle A, Stuermer A. Parallel Implementation of a Dynamic Overset Unstructured Grid Approach. ECCOMAS 2004, Jyväskylä (fi), 24.-28.0\%., 2004.

[22] Auerswald T, Weinreis C, Bange J, Raasch S, Radespiel R. Comparison of high resolution large-eddy simulations and synthetic turbulent wind fields. AIAA2010-1253, 2010. 



\title{
[B]
}

\section{Hybrid RANS/LES simulations of a three-element airfoil}

\author{
S. Reuß, T. Knopp, D. Schwamborn \\ Institute of Aerodynamics and Flow Technology, \\ DLR (German Aerospace Center) Göttingen, Germany
}

\author{
Published in: \\ Fu, S. Haase, W. Peng, S.-H. Schwamborn, D. (Eds.): \\ Progress in Hybrid RANS-LES Modelling, Notes on Numerical Fluid \\ Mechanics and Multidisciplinary Design Vol. 117, pp. 357-367, \\ Springer, 2012
}

\begin{abstract}
In this paper the Spalart-Allmaras based Delayed Detached Eddy Simulation (DDES [1]) and Improved Delayed Detached Eddy Simulation (IDDES [2]) are used to simulate the flow about an industrially relevant airfoil-configuration with deployed high-lift devices. Here, the potential advantage of the computationally very challenging hybrid approaches over pure RANS simulations in the case of incipient separation is investigated.
\end{abstract}

\section{Test case description}

The investigated DLR F15 three-element airfoil was developed in the DLR project LEISA as a two-dimensional cut through a generic aircraft wing [3]. It is one of the so-called application challenge test cases in the EU project ATAAC ${ }^{4}$. with a different point of focus this test case also considered, in other projects like the national German research project ComFliTe (Computational Flight Testing), the Garteur

${ }^{4}$ http://cfd.mace.manchester.ac.uk/ATAAC/WebHome 
Action Group $49^{5}$ and the German Research Council (DFG) project FOR1066 ${ }^{6}$.

Here, experimental data at a moderate angle of attack of $7^{\circ}$ are used, as the separation at the wind tunnel sidewalls is considered minor for low angles, and the flow can be regarded as almost two-dimensional. Measurements were performed in the low-speed wind-tunnel NWB at DLR Braunschweig at a Reynolds-number of $\mathrm{Re}=2 * 10^{6}$ based on the retracted chord length and a Mach-number of $\mathrm{Ma}=0.15$. The wind tunnel model has a chord length of $c=0.6 \mathrm{~m}\left(c_{\text {slat }}=20 \% c\right)$ and a span width of $2.8 \mathrm{~m}$. The experimental data used for validation consist of the mean pressure distribution in three spanwise sections and infrared measurements for the detection of the transition location. However, there are no experimental data available for mean velocity or Reynolds stresses, yet.

The geometry consists of a main wing element with deployed slat and flap. The inclination angles are $28.814^{\circ}$ for the slat and $38.296^{\circ}$ for the flap. The trailing edges of all elements except the lower slat edge are blunt. The main physical phenomena at the Reynolds-number considered are flow-generated airframe noise generated at the slat trailing edge and the separation on the flap at around $45 \%$ flap chord. The test case comprises several challenges to the numerical simulation: (1) to predict the noise that is generated in the slat cove, those turbulent structures must be accurately predicted that form in the shear layer between the recirculation region in the cove and the flow through the gap between slat and main wing ${ }^{7}$. (2) Behind the blunt trailing edge of the slat another shear layer is generated, which must be carefully captured to prevent unphysical interaction with the boundary layer on the main wing element. (3) Behind the trailing edge of the main wing element another shear layer develops. This shear layer together with the one stemming from the slat trailing edge influences the behavior of the flow over the flap. (4) Driven by an adverse pressure gradient, the flow separates at the flap, which turned out to be the major hurdle in the application of the hybrid RANS/LES approaches.

\section{Numerical approach}

The DLR TAU code [4] employed for this investigation is a finite volume solver for unstructured meshes providing several turbulence models, which have been heavily validated for many configurations, e.g. [5, 6, 7, 8]. In all simulations presented the one-equation Spalart-Allmaras RANS model was used as the basis. The SA based DDES-approach has been successfully applied to other testcases [9]. The equations are discretized with a central scheme with matrix dissipation. The time accurate computations are performed with dual time stepping and a three-stage low-storage Runge-Kutta scheme within each dual time step, employing three-level multi-grid to accelerate the convergence. Further speedup is gained through the use of parallel computations. Due to the large grid size in this test case a good parallel speedup could be obtained up to 2048 cores in the presented computations. This large number of cores was available for some time, when access to the supercomputer JUROPA of the "Hochleistungsrechenzentrum (HLRS) Jülich" was granted in a collaboration. These massively parallel computations reduced the computational time to a feasible

\footnotetext{
${ }^{5}$ http://www.garteur.org/Action\%20Group\%20posters/AD_AG_49\%20Poster\%20110320.pdf

${ }^{6}$ http://www.tu-braunschweig.de/ism/forschung/ag-sbsm/projekte/for 1066

${ }^{7}$ Currently ongoing PIV measurements will give further insight into these structures as well as about the point, where the shear layer impinges on the slat.
}

Fu et al. (Eds.): Progress in Hybrid RANS-LES Modelling, NNFM 117, pp. 357-367, Springer, 2012 
amount.

In [10] the coherence of the perturbation pressure over the spanwise extent is analysed. There, the authors write that the spanwise extent of the computational domain should be at least $80 \%$ of the slat chord length $c_{\text {slat }}$ in order to allow the solution in the slat cove and on the main wing element nose to become completely decorrelated if periodic boundary conditions are used. Here, with $c_{\text {slat }} 20 \%$ of $c$, resulting in a recommended span wise extent of $16 \% c$. On the other hand using twice the height of the separation region on the flap (2.5\% $c$ in the RANS simulations) is considered sufficient for the resolution of the separation.

The three-dimensional grid employed here was obtained by stacking a number of identical two-dimensional grids with constant step size in the spanwise direction (ydirection). This grid has been constructed with the hybrid grid generator CENTAUR and consists of about 200000 points in the $x z$-plane with 2000 points on the surfaces of all three elements and 45 cells normal to the wall in the structured layers. The first node above solid walls is located at a distance of about one in plus units. The cell size in the focus region, i.e. the slat cove and in the separation region above the flap, is about $0.14 \% c$. Using 64 layers of this grid in spanwise direction over a width of $9 \% c$ leads to a total number of $12.8 * 10^{6}$ points. With a retracted chord length of $0.6 \mathrm{~m}$ and a free-stream velocity of $50 \mathrm{~m} / \mathrm{s}$ one Convective Time Unit is $t_{\mathrm{CTU}}=0.012 \mathrm{~s}$; resolving one CTU by 600 steps the time step in the simulation was chosen as $2 * 10^{-5} \mathrm{~s}$.

To account for three-dimensional influences of the flow in the wind tunnel the incidence angle had to be adjusted in the present simulations. In a thorough precursor RANS study, the inflow conditions, in particular the angle of attack and the transition locations were adjusted to represent the experimental situation and to account for the wind tunnel wall effects. This study revealed the transition locations as a very sensitive parameter to the separation on the flap. Prescribing transition at the experimentally detected positions leads to an unphysical massive separation region on the flap, therefore transition locations were obtained through numerical prediction using the $e^{\mathrm{N}}$-method from the transition prediction module in the TAU code [11]. In figure 1, the pressure distributions from several SA-RANS simulations are compared. The green line shows the result from a fully turbulent RANS computation, i.e. no laminar regions are prescribed. The distribution is in good agreement with the experimental data, but the plateau in the pressure distribution is not quite captured. The streamlines show a clear separation region. The pressure distributions from two simulations with transition prescribed for all elements at angles of attack of $\alpha=5.0^{\circ}$ and $\alpha=6.0^{\circ}$ are given by the orange and red lines, respectively. It becomes obvious that a variation of the incidence angle can change the suction peaks on the slat and main wing element, but has no significant influence on the flap. In both cases no pressure plateau in the rear part of the flap can be observed. The distribution of the streamwise skin friction shows that the separation point on the flap is the same for both incidence angles. The streamlines indicate that prescribing transition on the flap leads to almost attached flow. The blue line shows the pressure distribution with transition prescribed on the slat and main wing only and a fully turbulent flap. Here the best agreement with the experimental data is achieved. The skin friction distribution shows that the separation is more upstream than in the fully turbulent simulation. Therefore, the hybrid calculations are performed with transition prescribed only on the slat and main wing at an angle of attack of $\alpha=6.0^{\circ}$. It is, however, not clear whether this optimization of the settings transfers directly

Fu et al. (Eds.): Progress in Hybrid RANS-LES Modelling, NNFM 117, pp. 357-367, Springer, 2012 

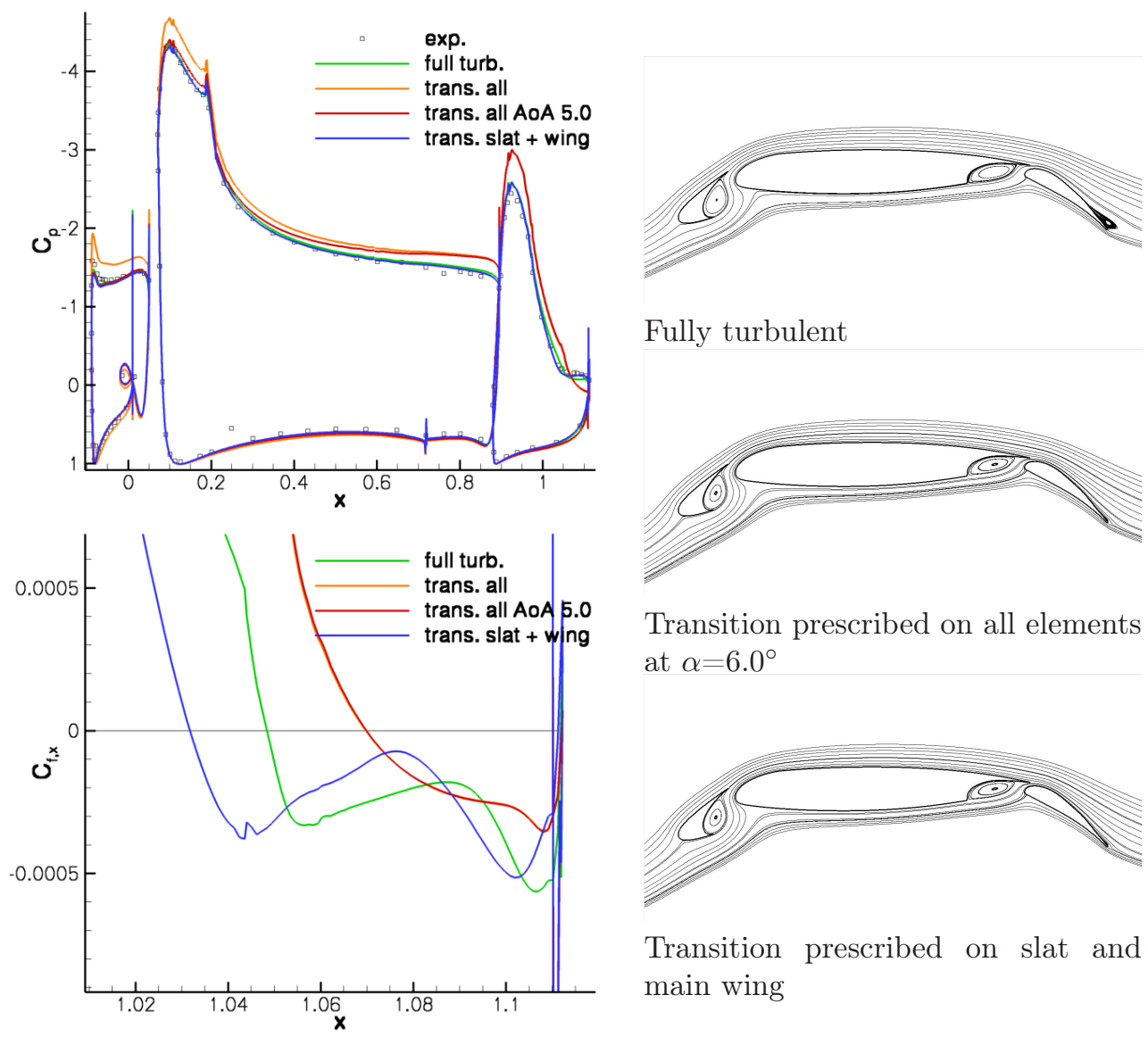

Fully turbulent

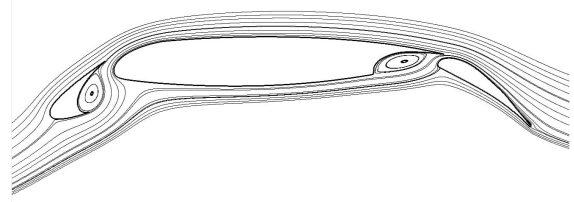

Transition prescribed on all elements at $\alpha=6.0^{\circ}$

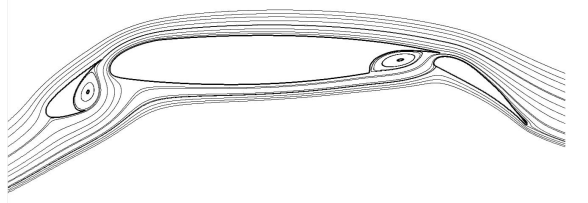

Transition prescribed on slat and main wing

Fig. 1: Pressure distribution, skin friction and streamlines for different transition settings obtained in precursor RANS simulations. Discussion of results

to the hybrid models, but it is too expensive to perform these studies using a hybrid RANS/LES model.

Several hybrid approaches were tested. The pressure distributions from all approaches are compared with RANS results with transition on all elements and with transition only on the slat and the main wing and with experimental data in figure 1 (left). On the right the streamwise component of the skin friction is shown. In table 1 the loads on the single elements in the presented simulations are compared. In the experimental data only a few pressure probes are located on each element, so these values are afflicted with a large error, as direct force measurements are not available.

In a first simulation a Spalart-Allmaras based DDES [1] was used [12]. The calculation was carried out for about $22 t_{\mathrm{CTU}}$. Figure 3 (left) shows the streamlines of the mean velocity field near the flap: with this approach the flow stayed attached. This can also be seen from the pressure distribution and the skin friction (fig. 2). Here, the suction peaks on all elements are too small and the pressure distribution deviates significantly from the experimental data and the RANS simulations. In the following we try to find an explanation for these observations.

Fu et al. (Eds.): Progress in Hybrid RANS-LES Modelling, NNFM 117, pp. 357-367, Springer, 2012 

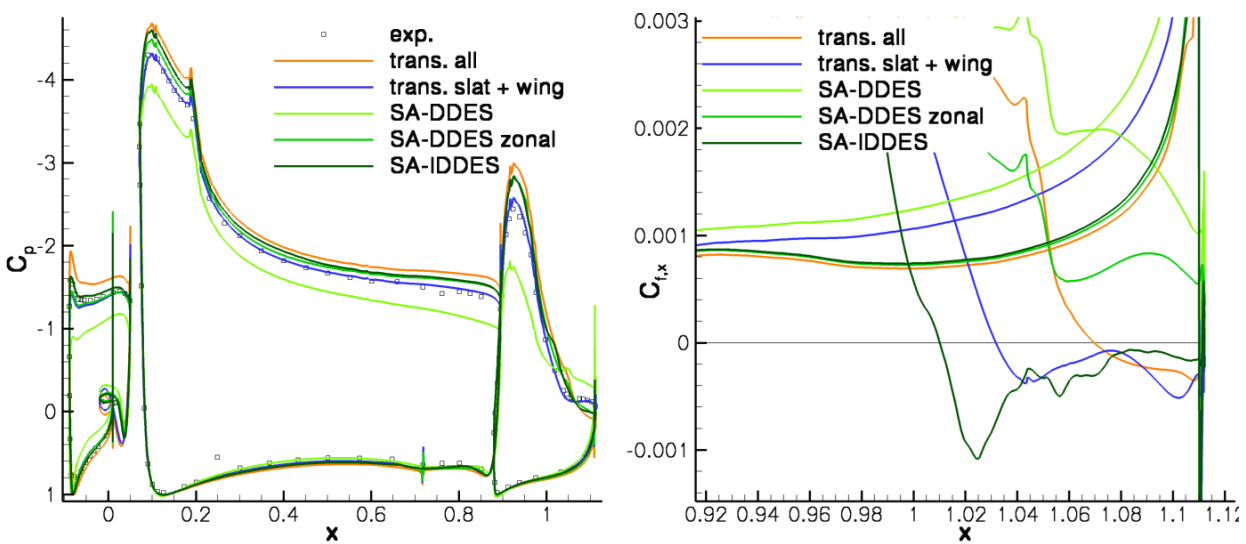

Fig. 2: Comparison of the pressure distribution from precursor RANS simulations with SADDES, zonal SA-DDES and SA-IDDES, all at a corrected angle of attack of $6^{\circ}$

\begin{tabular}{|l|l|l|l|l|l|l|}
\hline & EXP & RANS & $\begin{array}{l}\text { trans. all } \\
\text { RANS }\end{array}$ & $\begin{array}{l}\text { trans. } \\
\text { s+w } \\
\text { DDES }\end{array}$ & $\begin{array}{l}\text { Zonal } \\
\text { DDES }\end{array}$ & IDDES \\
\hline Slat & 0,202 & 0,251 & 0,210 & 0,207 & 0,225 & 0,221 \\
\hline Wing & 1,872 & 2,076 & 1,925 & 1,986 & 1,932 & 2,013 \\
\hline Flap & 0,316 & 0,374 & 0,325 & 0,354 & 0,402 & 0,351 \\
\hline$\sum$ & 2,391 & 2,701 & 2,459 & 2,547 & 2,559 & 2,585 \\
\hline
\end{tabular}

Tab. 1: Comparison of the loads on the single elements from precursor RANS simulations with SA-DDES, zonal SA-DDES and SA-IDDES, all at a corrected angle of attack of $6^{\circ}$

An instantaneous snapshot of the contours of vorticity is shown in figure 4 (top). With respect to the flow in the slat cove the vortical structures are quite coarse. Behind the slat trailing edge the free shear layer breaks up and the unsteady structures penetrate into the boundary layer on the main wing. This disturbance of the formal RANS layer is persistent all the way over the main wing element even very close to the wall, where a steady-state RANS solution would be expected.

Associated with these unsteady events, additional turbulent kinetic energy is transported into the boundary layer and at the same time it seems that the turbulence production of the RANS model is increased there, leading to an overly increased and
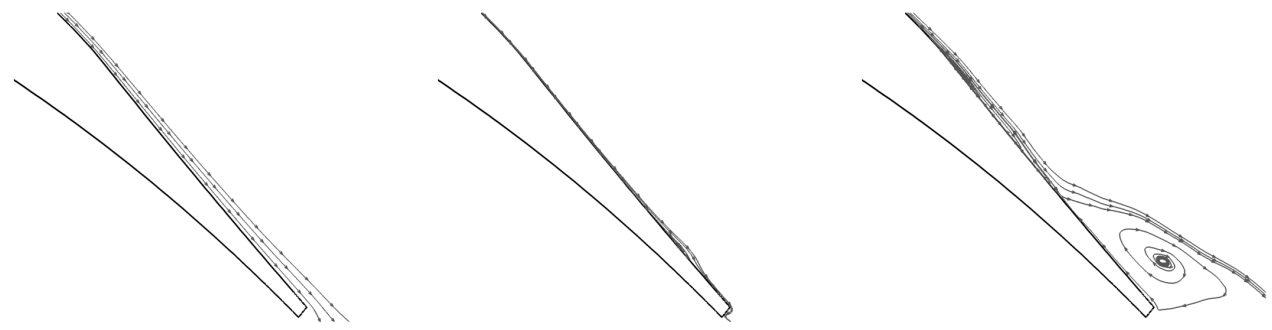

Fig. 3: Streamlines on the flap: SA-DDES (left), zonal SA-DDES (middle) and SA-IDDES

Fu et al. (Eds.): Progress in Hybrid RANS-LES Modelling, NNFM 117, pp. 357-367, Springer, 2012 


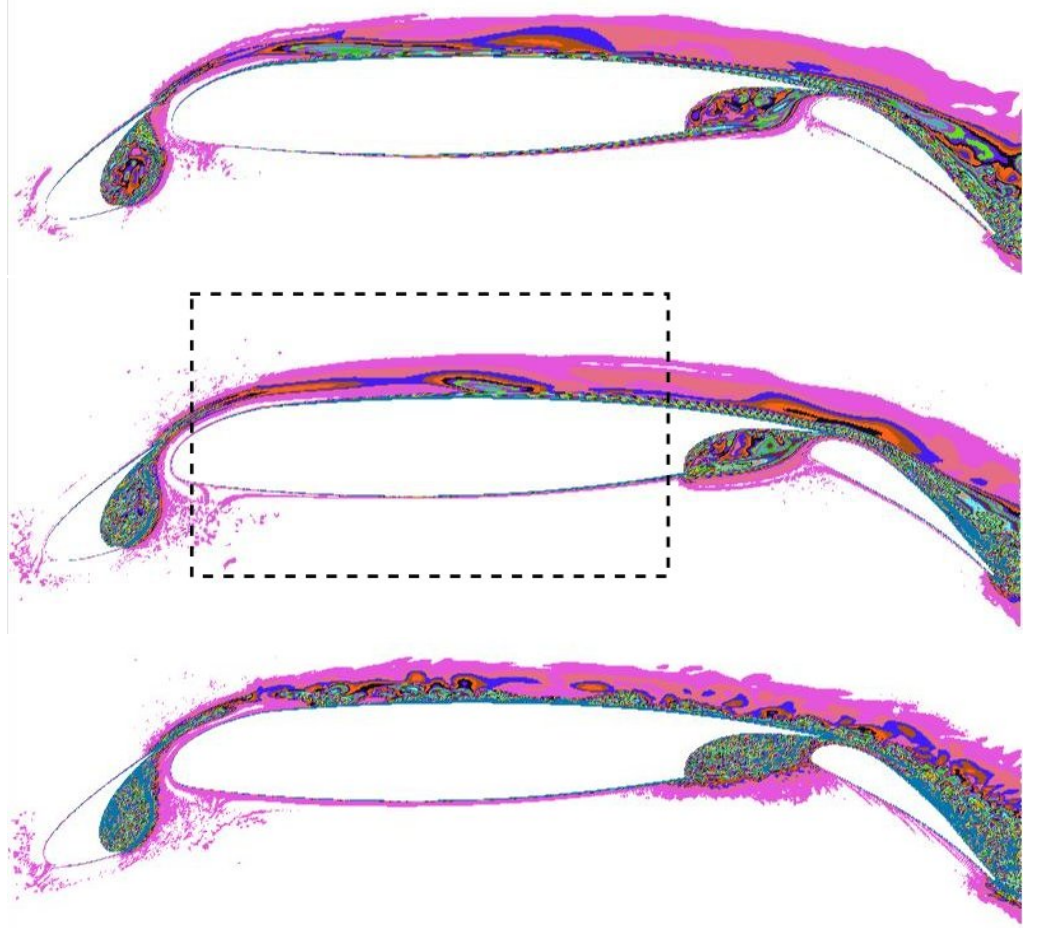

vorticity: $\quad 10002000300040005000600070008000$

Fig. 4: Instantaneous contours of vorticity: SA-DDES (top), zonal SA-DDES (middle) and SA-IDDES (bottom)

probably unphysical turbulent shear stress in the xz-plane. The total shear stress, given by

$$
\left(\tau_{x z}\right)_{\text {total }}^{\text {turb }}=\underbrace{\mu_{t}\left(\frac{d u}{d z}+\frac{d w}{d x}\right)}_{\left(\tau_{x z}\right)_{\text {modelled }}^{\text {turb }}}-\underbrace{\rho\left\langle u^{\prime} w^{\prime}\right\rangle}_{\left(\tau_{x z}\right)_{\text {resolved }}^{\text {turb }}},
$$

is responsible for the wall normal transport of momentum in the boundary layer. A formal evaluation of $\tau_{x z, t o t}$ was performed and the contours of the different approaches are compared in figure 5 (In the RANS computation the resolved part of the shear stress vanishes). In the SA-DDES the total turbulent shear stress on the rear part of the main wing and the flap is massively overpredicted compared to the RANS simulation. Since in the SA-DDES the modeled turbulent shear stress is, as expected, much lower than in the RANS computation (not shown here), the main contribution comes from the resolved turbulent shear stress. This increased total shear stress leads to a higher transport of momentum towards the wall over the flap, which prevents the separation on the flap.

Based on the first DDES results a redesign of the grid was performed before the following simulations were started. The total number of points in the xz-plane was not changed significantly, but more points were clustered in the slat cove region and

Fu et al. (Eds.): Progress in Hybrid RANS-LES Modelling, NNFM 117, pp. 357-367, Springer, 2012 


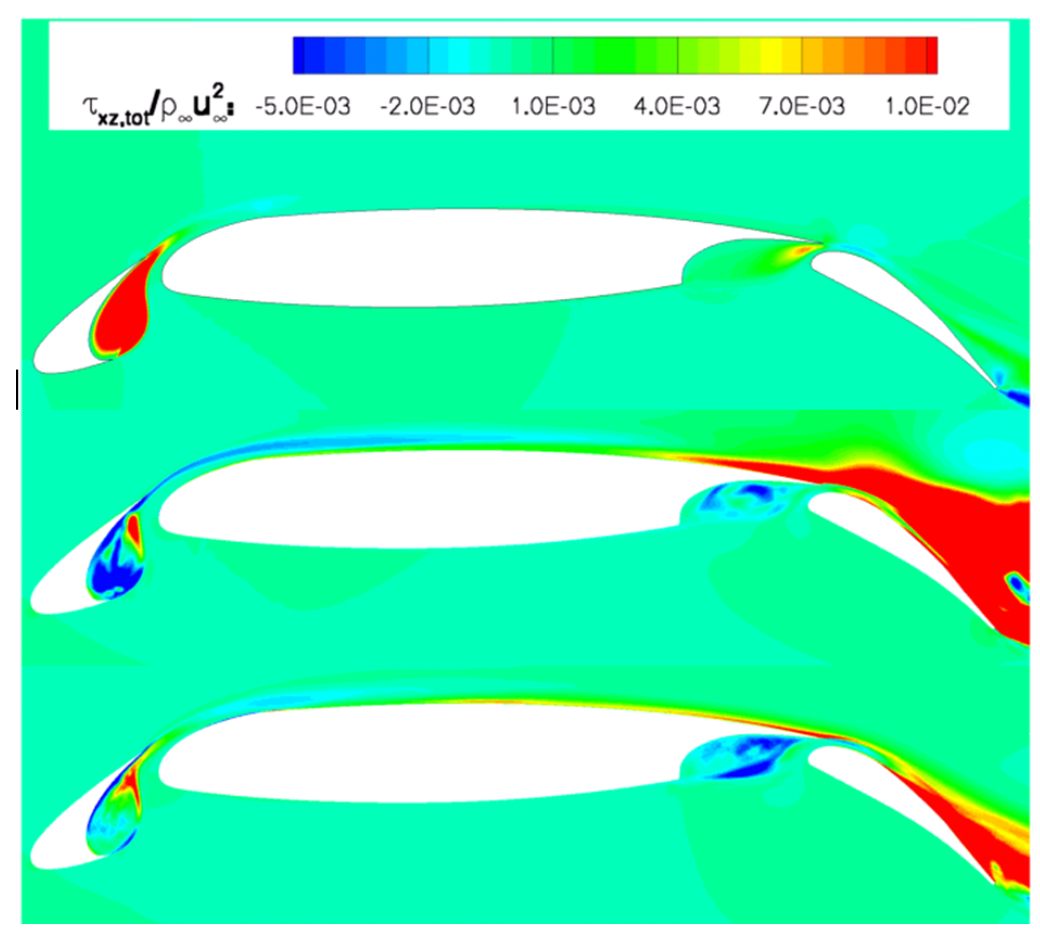

Fig. 5: Contour of turbulent shear stress in xy-plane: RANS (top), SA-DDES (middle) and SA-IDDES (bottom)

above the flap. To prevent the unphysical interaction of the slat wake with the boundary layer on the main wing, two strategies were followed: In a zonal approach the RANS mode was enforced around the main wing element. This simulation ran for about $30 t_{\mathrm{CTU}}$ and then was stopped as the situationwith regard the separation on the flap did not improve much. In figure 3 the streamlines for the zonal DDES show a very flat recirculation bubble in the instantaneous ${ }^{8}$ velocity field, after which the flow reattaches. The pressure distribution (fig. 2) is in better agreement with RANS and experiments than the non-zonal approach. It can be seen, that the missing separation on the flap leads to higher suction peaks on the slat and main wing. Because of the missing separation the plateau on the flap is not reproduced, accordingly, this simulation was stopped even before converged RMS values were available. Thus the turbulent shear stress was not evaluated for the zonal approach.

In the middle part of figure 4, the vorticity distribution for the zonal approach is shown, with dashed lines indicating the boundaries of the enforced RANS region. The redesigned grid has a positive effect on the resolved structures in the slat cove. Even though the situation is formally the same as in the non-zonal DDES up to the interface of the enforced RANS zone, the structures are much smaller than before. Once the free shear layer behind the slat trailing edge enters the RANS region, the vortical structures do not die out immediately. Even though the shear layer is preserved longer compared to the non-zonal DDES, it breaks up finally and again penetrates into the near wall region of the boundary layer, leading to a somewhat similar situation as in the standard DDES case on the less resolved grid.

${ }^{8}$ The averaging period was not long enough to provide statistically converged results

Fu et al. (Eds.): Progress in Hybrid RANS-LES Modelling, NNFM 117, pp. 357-367, Springer, 2012 
Secondly, we consider IDDES [2] as alternative approach. IDDES was designed to deal with unsteadiness in boundary layers and should as such be an ideal candidate for the interaction between a shear layer and an underlying boundary layer, sufficient resolution provided. This simulation was run for the longest time until statistical convergence was reached after about $100 t_{\mathrm{CTU}}$. The mean and RMS values were taken over the last $40 t_{\mathrm{CTU}}$. The suction peaks in the pressure distribution (fig. 2) are higher than in the zonal DDES simulation but a small plateau can be seen at the rear of the flap. This simulation is the first hybrid approach that results in separated flow on the flap as the streamlines of the mean velocity show (fig. 3 right). As in the zonal approach, first a flat recirculation bubble occurs, but behind that the flow separates, even though the separation region is smaller than that predicted in the RANS simulation. The beginning of the recirculation bubble is upstream of the experimentally expected position and also earlier than predicted by the RANS simulation, as the skin friction distribution illustrates.

The vorticity contours in figure 4 show again a break up of the shear layer behind the slat. The resolved structures in the slat cove as well as above the flap are much smaller than in the zonal approach. Also, the vortical structures that travel along the main wing element are much smaller and they are preserved all the way to the trailing edge. That this behavior is more physical might also be concluded from the turbulent shear stress, shown at the bottom of figure 5, which is much smaller than in the DDES. That it is still higher than in the RANS computation, might indicate that the grid is not fine enough to resolve the vortical structures in the boundary layer.

In figure 6 the Fourier transformation of the time signal of the lift coefficients of the zonal DDES and the IDDES are compared. In both cases the standard deviation is in the order of 0.02 which is about $1 \%$ of the mean value. Note, that the two spectra are not exactly comparable due to the much shorter signal length available for the DDES. Even though the lift value is integrated over the whole surface, the influence of the small scale structures can be seen. Both spectra show a peak near the frequency of $750 \mathrm{~Hz}$. In the zonal DDES this peak is slightly shifted to a lower frequency. In the spectrum of the IDDES more peaks can be observed. These are caused by the small scale structures in the wakes of the upstream elements that interact with the respective downstream elements.

In figure 7 (top), the resolved turbulent kinetic energy that is obtained in the IDDES simulation is compared with the modeled turbulent kinetic energy predicted with a $\mathrm{k} \omega$-RANS simulation. Above the flap the level of resolved energy is of the same order of magnitude. However, in the slat and wing coves, the resolved turbulent kinetic energy in the IDDES is higher than the modeled one in the RANS simulation. Figure 7 (bottom) presents the turbulent length scale based on the k $\omega$-RANS simulation as percentage of the chord length. The length scale in the slat cove is about $l=0.07 \% c$ and in the shear layer behind the slat even $l=0.04 \% c$. With a cell size of $0.14 \% c$ in the presented simulations the resolution is of the same order of magnitude. With the approach of generating the three-dimensional grid by stacking two-dimensional grids in spanwise direction, a higher resolution seems not feasible.

Fu et al. (Eds.): Progress in Hybrid RANS-LES Modelling, NNFM 117, pp. 357-367, Springer, 2012 


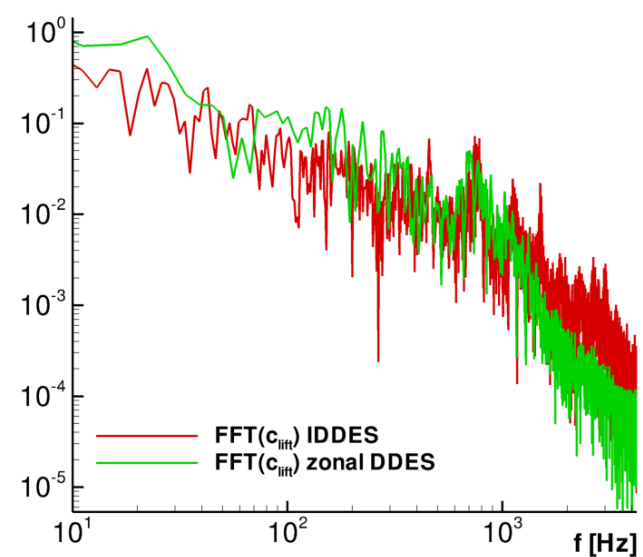

Fig. 6: fft-analysis of the lift coefficient time signal from the zonal DDES and the IDDES
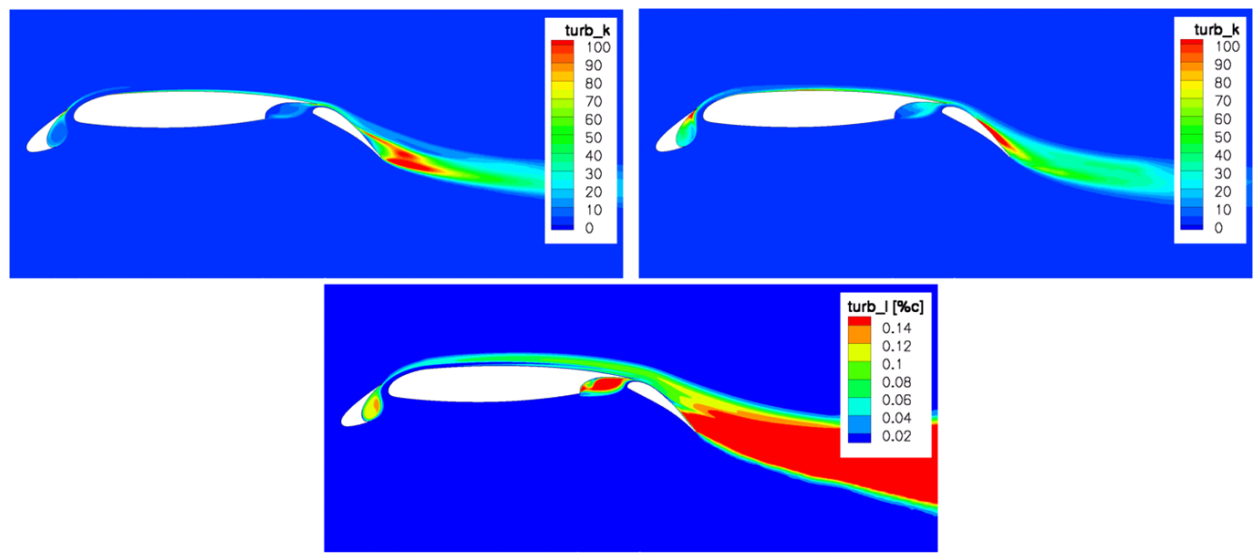

Fig. 7: Turbulent kinetic energy from $\mathrm{k} \omega$-RANS (top left) and from SA-IDDES computation (top right) and turbulent length scale $l$ (bottom)

\section{Conclusion}

In this paper three hybrid RANS/LES simulation were presented: a SA-DDES, a zonal SA-DDES and a SA-IDDES. Within the scope of this work, it was not possible, to achieve a statistically fully converged state within all simulations. In a comparison of the mean pressure distributions obtained in the hybrid calculations with the basic RANS results the latter coincide best with the experiments. This is however inherent in the standard procedure of calibrating the inflow conditions using RANS. To assess the differences observed in the three hybrid simulations streamlines and the instantaneous vorticity were compared. Further insight was gained by the evaluation of the total turbulent shear stress. In the SA-DDES an unphysical interaction of the slat-wake with the boundary layer on the main wing element can be observed. Disturbances that penetrate into the formal RANS area that is shielded by the delay function are neither damped correctly nor are they resolved. Thus, the

Fu et al. (Eds.): Progress in Hybrid RANS-LES Modelling, NNFM 117, pp. 357-367, Springer, 2012 
RANS-model produces an erroneous eddy viscosity that is convected downstream the main wing and leads to an increased wall normal shear stress in the region above the flap. This prevents the flow from separating at the flap. The DDES approach is therefore considered not to be able to deal with the confluence of shear layers with boundary layers. In order to prevent or resolve this interaction, respectively, a zonal SA-DDES and a SA-IDDES simulation were conducted on the same grid. It was shown, that enforcing the RANS mode where the interaction occurs, did not help to prevent unsteady structures from the shear layer penetrating into the boundary layer. This is probably because the numerical dissipation on the relatively fine grid (for RANS) does not dissipate the resolved eddies fast enough. The flow stayed attached on the flap in the zonal SA-DDES, assumedly due to the same error that is introduced in the non-zonal SA-DDES. With a much coarser grid in the enforced RANS region, a similar zonal approach is used successfully in [13], here the separation is predicted. Therefore it is concluded, that a zonal approach together with a grid that is adapted to match the prescribed RANS zones can be recommended from an engineering point of view if the focus of a simulation is on localized regions while unsteady features in other regions can remain unresolved. The SA-IDDES was the only one of the presented approaches that was able to predict the separation on the flap. This demonstrates that this approach is able to predict the interaction of the slat wake with the boundary layer on the main wing in a physically correct way. However, the evaluation of the data indicates that the flow was still underresolved. It is concluded that the IDDES approach is a very promising approach, but that a very highly resolved grid is needed. The observed problems in the presented hybrid RANS/LES simulations are further investigated. It seems, however, not feasible to achieve a globally much finer resolution. Therefore, (1) the noise generated in the slat cove and (2) the separation on the flap are now investigated separately, in order to reduce the computational effort and to distinguish clearly the effects. Two different grids are used with a very high resolution in the streamwise as well as in the spanwise direction, adequate for a wall-modeled LES, in the respective region where the point of focus lies. The rest of the computational domain is resolved with a much coarser grid, and there the RANS mode is enforced. The detailed understandings from these new simulations will help to reach the goal of hybrid simulations with RANS quality as a lower limit for industrially relevant testcases.

\section{References}

[1] P. R. Spalart, S. Deck, M.L. Shur, K.D. Squires, M.K. Strelets, and A. Travin. A New Version of Detached-eddy Simulation, Resistant to Ambiguous Grid Densities. Theoretical and Computational Fluid Dynamics, 20(3):181-195, 2006.

[2] M.L. Shur, P.R. Spalart, M.K. Strelets, and A. Travin. A hybrid RANS-LES approach with delayed-DES and wall-modelled LES capabilities. International Journal of Heat and Fluid Flow, 29(6):1638-1649, 2008.

[3] J. Wild and M. Pott-Pollenske. An integrated design approach for low noise exposing high-lift devices. AIAA 2009-3101, 2009.

[4] D. Schwamborn, T. Gerhold, and R. Heinrich. The DLR TAU-Code: recent applications in reaserach and industry. in proceedings of ECCOMAS CFD 2006, Wesseling, P. Oñate, E. Périaux, J. (eds.), The Netherlands, TU Delft, 2006.

Fu et al. (Eds.): Progress in Hybrid RANS-LES Modelling, NNFM 117, pp. 357-367, Springer, 2012 
[5] D. Schwamborn, A. Gardner H. von Geyr, A. Krumbein, H. Lüdeke, and A. Stürmer. Development of the TAU-Code for aerospace applications. in 50th NAL International Conference on Aerospace Science and Technology, 2008.

[6] S. Melber-Wilkending. Aerodynamics of the Wing/Fuselage Junction at an Transport Aircraft in High-lift Configuration. in 16th AG STAB/DGLR Symposium, 2008.

[7] R. Rudnik. Stall Behaviour of the EUROLIFT High Lift Configurations. AIAA 2008-836, 2008.

[8] S. Crippa, S. Melber-Wilkending, and R. Rudnik. DLR Contribution to the First High Lift Prediction Workshop. AIAA 2011-938, 2011.

[9] D. Schwamborn and M.K. Strelets. ATAAC - an EU-Project Dedicated to Hybrid RANS/LES Methods. Invited paper at 4 th Symposium on Hybrid RANS/LES Methods, Beijing, Sept. 2011, Notes on Numerical Fluid Mechanics and Multidisciplinary Design, Springe, 2011.

[10] D. P. Lockard and M.M. Choudhari. Noise Radiation from a Leading-Edge Slat. AIAA 2009-3101, 2009.

[11] A. Krumbein and N. Krimmelbein. Navier-Stokes High-Lift Airfoil Computations with Automatic Transition Prediction using the DLR TAU Code. in Notes on Numerical Fluid Mechanics and Multidisciplinary Design, Vol. 96, New Results in Numerical and Experimental Fluid Mechanics VI, Springer Verlag, Berlin-Heidelberg-New York, pp. 210-218, 2007.

[12] D. Jakubek. Parameterstudien für numerische RANS- und DES-Verfahren für ein Drei-Element-Profil in Hochauftriebskonfiguration mit dem DLR TAU-Code. DLR-IB 224-2010 A39, 2010.

[13] S. Deck. Zonal-Detached-Eddy Simulation of the Flow Around a High-Lift Configuration. AIAA Journal, 43(11), 2005.

Fu et al. (Eds.): Progress in Hybrid RANS-LES Modelling, NNFM 117, pp. 357-367, Springer, 2012 



\title{
$[\mathrm{C}]$
}

\section{Investigation of the Resolution Requirements for a Hybrid RANS/LES Simulation of a Multi-Element Airfoil}

\author{
S. Reuß, T. Knopp, D. Schwamborn \\ Deutsches Zentrum für Luft- und Raumfahrt, \\ Center for Computer Applications in AeroSpace Science and Engineering $\mathrm{C}^{2} \mathrm{~A}^{2} \mathrm{~S}^{2} \mathrm{E}$, \\ Institute of Aerodynamics and Flow Technology, \\ Bunsenstrasse 10, \\ 37073 Goettingen
}

\section{Published in:}

Kroll, N. Radespiel, R. v. d. Burg, J.W. Sørensen, K. (Eds.): Computational Flight Testing, Notes on Numerical Fluid Mechanics and Multidisciplinary Design Vol. 123, pp. 43-57, Springer, 2013

\begin{abstract}
This work is dedicated to the investigation of the resolution requirements for hybrid RANS/LES simulations for aerodynamic flows at high-lift. First, results of a local DDES for a limited section of a high-lift wing with deployed slats and flaps of a full-aircraft configuration are presented. Based on the resolution of this simulation the computational effort for a hybrid RANS/LES simulation of the complete high-lift wing is estimated. Since this estimate results in prohibitively high costs, the focus is shifted to the scale-resolving simulation of a quasi two-dimensional segment of a three-element wing for the further investigations. Three approaches, a zonal DDES, a global DDES and an IDDES, are presented and the last one is evaluated with respect to the resolution of the boundary layers as well as the free shear layers.
\end{abstract}




\section{Introduction}

The idea of hybrid RANS/LES models is to reduce the computational effort compared to a pure LES computation by treating attached boundary layers in RANS mode. RANS models are reliable if applied to attached flows with a small or moderate pressure gradient, while hybrid models like the Detached Eddy Simulation (DES) model [1] have been applied with great success to test cases with massive separation, fixed by the geometry. In these cases a clear superiority over RANS models has been demonstrated [2]. Flows with smaller separation, caused by an adverse pressure gradient, are still very challenging for the numerical simulation. The benefit of hybrid RANS/LES models for these cases is under investigation.

The reduction of airframe-noise caused by the high-lift devices is one important contribution to the reduction of the overall noise of an aircraft. The direct access to the instationary sources of aerodynamic noise is another motivation for a hybrid RANS/LES simulation.

When the ComFliTe project was started, the idea was to assess a local hybrid RAN$\mathrm{S} / \mathrm{LES}$ approach for a section of a high-lift wing of a full-aircraft in landing configuration near maximal lift $C_{l, \max }$. At this point there were several open questions:

1. What are the expected computational costs for such a simulation?

2. Which model from the DES family is best suited for high-lift cases?

3. Which requirements for a proper mesh resolution can be given?

The outline of this paper is as follows: In Sec. 2 the numerical method is shortly described. In Sec. 3 results from a local DDES of a section of a high-lift wing with a small spanwise extent embedded into a global RANS simulation of a full-aircraft configuration with focus on the aeroacoustics in the slat cove are presented. These are used in Sec. 4 to estimate the computational effort for a local DDES for the complete high-lift wing. With this estimate an answer to the first question could be given: it is not feasible to perform a zonal DDES for a complete high-lift wing of a full-aircraft configuration with the currently available computing resources and the budget within the ComFliTe project. Therefore, in order to find answers to the remaining open questions, the focus of the work was redefined and a three-element airfoil with periodic boundary conditions in the span-wise direction was chosen as the new test case. This configuration is described in Sec. 5. The results from several hybrid simulations are shown in Sec. 6 and the evaluation of the grid resolution is presented in Sec. 7. In the last section conclusions are drawn and an outlook is given.

\section{Numerical method}

All simulations were performed with the DLR TAU code [3], a compressible finite volume solver for the Navier-Stokes equations on unstructured meshes. The timeaccurate simulations employ a dual time-stepping scheme with a three-stage lowstorage Runge-Kutta scheme. The spatial discretization uses a central scheme with artificial dissipation of matrix type. The convergence is accelerated by a $3 \mathrm{w}$ multi-grid

Kroll et al. (Eds.): Computational Flight Testing, NNFM 123, pp. 43-57, Springer, 2013 
scheme. Different turbulence models are implemented for the closure of the ReynoldsAveraged NS-equations and based on these RANS models different variants of the hybrid RANS/LES models are available. The different variants can be distinguished by the function that switches from RANS to LES. In the original DES model [1] the switching is based on the size of the grid cells, whereas the Delayed DES (DDES) [4] variant provides a shielding function that is designed to ensure the RANS mode in the whole boundary layer independent of the grid. The Improved DDES (IDDES) [5] model additionally offers a mode that allows for wall modeled LES, resolving the outer part of the boundary layer.

All hybrid simulations that are presented here, are based on the Spalart-Allmaras turbulence model, utilizing the DDES as well as the IDDES model.

\section{Local DDES of a High-Lift Wing}

The full-aircraft configuration is a model of the A320 wing-body with nacelles and deployed slats and flaps. The wind-tunnel model has a half span width of $1.08 \mathrm{~m}$ and a mean chord length of $c_{\text {mean }}=0.224 \mathrm{~m}$. Experiments were performed at a Mach number of $M a=0.2$ and a Reynolds number of $R e=1.34 \times 10^{6}$ at an angle of attack of $\alpha=4^{\circ}$. The experimental data for this test case were obtained within the research project HICON [6]. One of the objectives of the project was to reduce the noise emissions of aircraft through innovative high-lift configurations. Therefore, the spectra emitted by the high-lift devices of a wind-tunnel model of an A320 were measured with microphone arrays. Additionally, the mean pressure distributions were taken in several span-wise positions.

The objective of the numerical investigation was to assess the influence of the model slat tracks on the emitted spectra. Therefore, a single model slat track, located at $60 \%$ span width, was included in the geometry. The aim of this work was to compare the noise that is generated by the model slat track to the noise that is emitted by a real slat track.

In order to obtain information about the instationary sources of noise, a local DDES was performed: the scale-resolving approach was used in the vicinity of the slat track while in the major part of the computational domain the RANS approach was used. To provide a mesh for this zonal approach, an appropriate hybrid grid for a RANS simulation with about $30 \times 10^{6}$ points was used as a basis. This basic mesh was refined in a region around the track of about $7 \%$ span-wise extent. The refined grid consisted of $80 \times 10^{6}$ points, with all additional $50 \times 10^{6}$ points located in the focus region, the wake of the slat track. A view of the region with the model slat track is presented in Fig. 1. The dark area on the main wing indicates the surface of the refined region for the zonal approach.

Spatial Resolution Requirements for the spatial resolution can be estimated by the measured frequencies: The acoustical data range from $f_{0}=2000 \mathrm{~Hz}$ to $f_{1}=$ $40,000 \mathrm{~Hz}$. With a speed of sound of about $a=343 \mathrm{~m} / \mathrm{s}$ at a temperature of $T=293 \mathrm{~K}$ and a free-stream velocity of $u_{\infty}=68 \mathrm{~m} / \mathrm{s}$ the distance that one acoustic wave travels per acoustic oszillation period is $\ell=u_{\infty}+a / f_{1} \approx 0.01 \mathrm{~m}$. To resolve this distance appropriately about 30 points are needed. Therefore, the cell size in the focus region should be about $\Delta_{x}=3.33 \times 10^{-4} \mathrm{~m}$.

Kroll et al. (Eds.): Computational Flight Testing, NNFM 123, pp. 43-57, Springer, 2013 


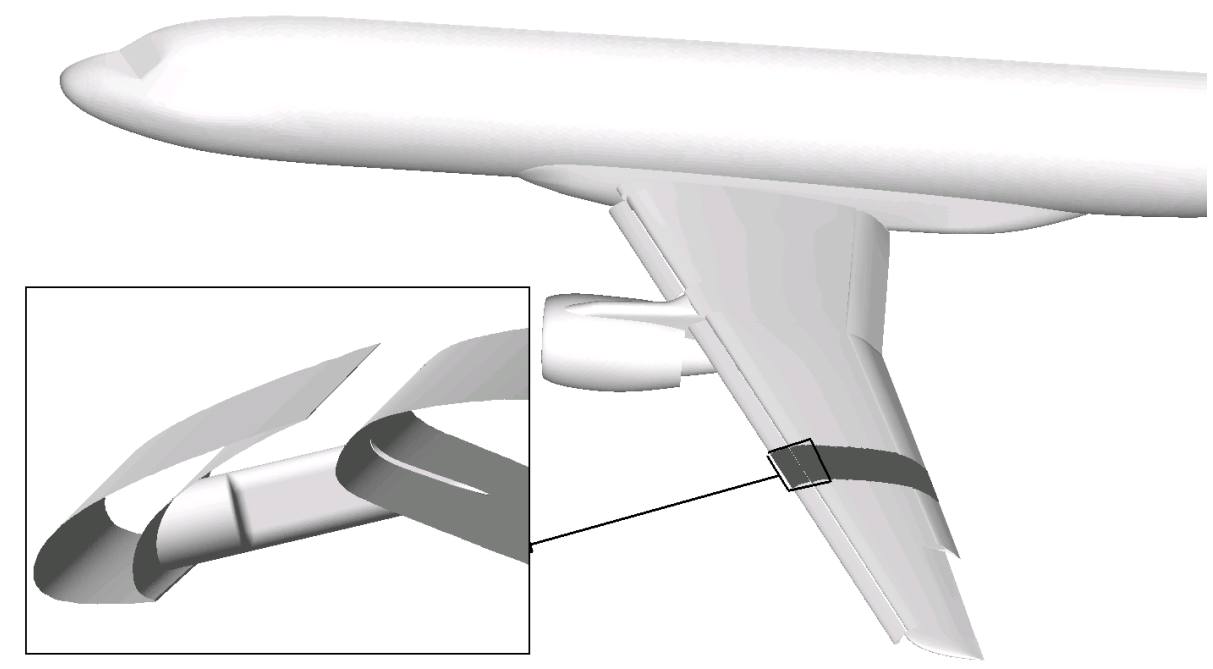

Fig. 1: Focus region of the local DDES of a full-aircraft configuration in high-lift with a single slat track

The refined grid was generated using the CENTAUR software. CENTAUR generates hybrid grids with hexahedral or prismatic cells in the near wall regions and tetrahedral cells in the farfield. The grid generation is controlled by sources that define the cell size in different regions. While on the one hand this process needs little interaction from the user, on the other hand no full controll over the shape of the elements is possible. The average cell size in the stream-wise and span-wise direction in the focus region is $\Delta_{x}=\Delta_{y}=3.6 \times 10^{-4} \mathrm{~m}$. The average number of surface points in a stream-wise cut through the wing is 2600 . In the wall-normal direction the number of points in the structured layers is 30 .

The generation of the refined grid was very time-consuming with one run lasting several days. Therefore, it was not possible to perform many iterations of the grid generation process, so that finally a compromise had to be made regarding the geometrical quality of the grid elements: unfavorable acute-angled and skewed grid cells could not be avoided completely. These can deteriorate the convergence rate of a simulation considerably, which was the case in the present simulations. So, in order to ensure an appropriate convergence per physical time step, the number of inner iterations had to be almost doubled compared to typical values from other hybrid RANS/LES simulations (e.g. those presented later on), leading to an increased computational effort for each physical time step.

Temporal Resolution The time step that is necessary for the resolution of the aeroacoustics can also be estimated from the measured frequencies. In order to resolve one period of the highest frequency of $f=40,000 \mathrm{~Hz}$ with about 30 time steps, the time-step size should be about $\Delta t_{\text {acous }} \approx 1 \times 10^{-6} \mathrm{~s}$. A similar estimate is obtained if the time is computed as the ratio between cell size $\Delta_{x}$ and sum of far field velocity $u_{\infty}$ and speed of sound $a$. However, if the focus is not on the aeroacoustics the estimate of the time step can be solely based on the free stream velocity $\Delta t=\Delta x / u_{\infty} \approx 5 \times 10^{-6} \mathrm{~s}$. In order to get physically meaningful spectra

Kroll et al. (Eds.): Computational Flight Testing, NNFM 123, pp. 43-57, Springer, 2013 
several periods of the lowest frequencies must be simulated. 100 periods of the $2000 \mathrm{~Hz}$ frequencies would result in $0.05 \mathrm{~s}$ simulation time. This would all together result in 50,000 physical time steps using $\Delta t_{\text {acous }}$. However, in order to get converged mean-flow and turbulent statistics a certain number of Convective Time Units (CTU), $t_{C T U}=\ell / u_{\infty}$, is needed as well, where $\ell$ is a characteristic length of the geometry, e.g. $\ell=0.253 \mathrm{~m}$ the averaged chord length of the airfoil in the refined region. Then $t_{C T U}=0.0037 \mathrm{~s}$ is the time that it takes the flow to pass the characteristic length. In order to get accurate second order statistics, $50 t_{C T U}$ are usually necessary which leads to 185,000 physical time steps using $\Delta t_{\text {acous }}$.

Parallel speedup With this large number of grid points the region of linear speedup extends to large numbers of processors. In the current case the computations were performed on 2048 cores of the DLR $\mathrm{C}^{2} \mathrm{~A}^{2} \mathrm{~S}^{2} \mathrm{E}$ cluster. At the time of the computations this was one third of the available resources of the cluster. The speed of the computation was almost doubled through doubling the number of processors.

With this massive parallel computational effort about 20 minutes of real time were needed to simulate one physical time step. Simulating 50,000 physical time steps would then have taken 694 days which was beyond the scope of the resources that could be acquired for this work. Therefore, it was decided to reduce the temporal resolution by a factor of 10 . With the reduced number of required time steps of 5,000 this still lead to a real simulation time of 69 days without interruption.

A scale-resolving hybrid RANS/LES simulation starts with an initial transient phase. In this phase the generation of turbulent content builds up. This phase must be overcome before reliable statistics can be computed and it is not clear a-priori how long it lasts, but at least several convective times $t_{C T U}$ are needed. An indicator for a fully developed turbulent flow are converged mean values.

During the simulations of the A320 some uncertainties aroused from other hybrid RANS/LES simulations of airfoil flows, that were conducted at the same time. These and limited computational resources led to the decision to stop the simulations prematurely. Even though the statistical evaluation of the results was not possible with the limited data available, still relevant insights into the computational effort of a such a scale-resolving simulation could be gained.

A representative qualitative result is presented in Fig. 2, depicting an iso-surface of the $Q_{i n v}$-criterion:

$$
Q_{i n v}=\frac{1}{2}\left[|S|^{2}+|\Omega|^{2}\right]>0
$$

with $S=\frac{1}{2}\left[\nabla \boldsymbol{u}+(\nabla \boldsymbol{u})^{T}\right]$ and $\Omega=\frac{1}{2}\left[\nabla \boldsymbol{u}-(\nabla \boldsymbol{u})^{T}\right]$, which is an indicator for a vortex, where the norm of the vorticity tensor dominates that of the rate of strain. The criterion can be used to visualize the small scale structures that form in the wake of the slat track. The span-wise velocity component $v$ is used to demonstrate the three-dimensional nature of the structures. It can be clearly seen that the major vortical structure over the main wing originates from the slat track, which is itself not visible in the picture.

Kroll et al. (Eds.): Computational Flight Testing, NNFM 123, pp. 43-57, Springer, 2013 


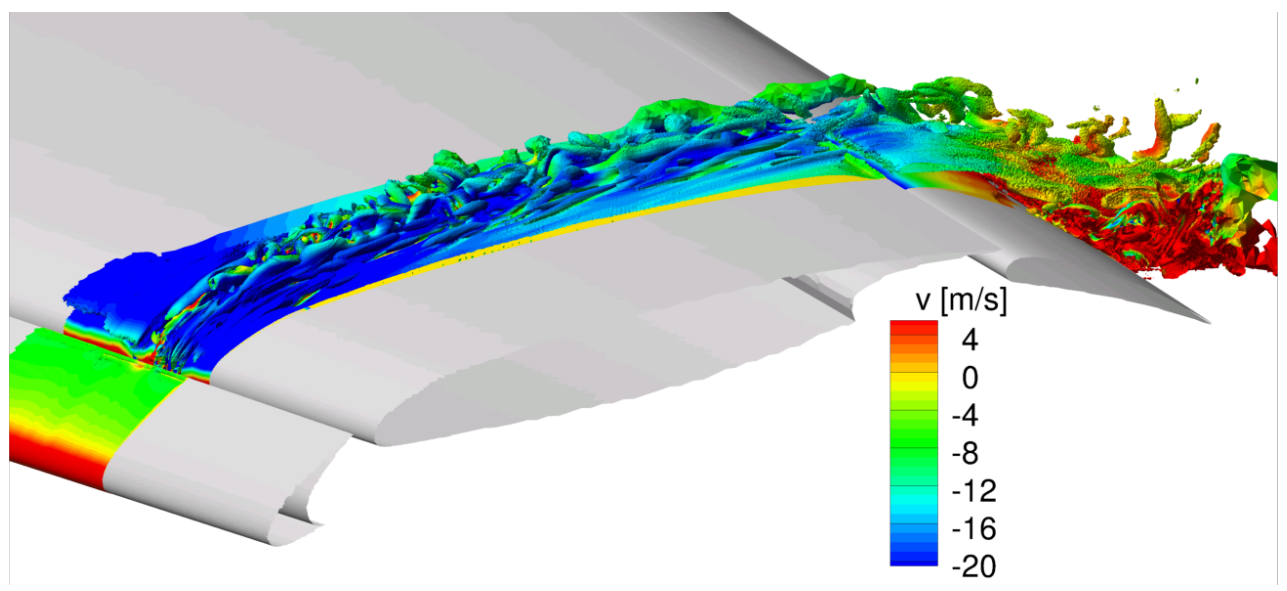

Fig. 2: Iso-surface of the $Q_{i n v}$-criterion to visualize the turbulent structures in the wake of the slat track, colored with the span-wise velocity component $v$.

\section{Estimation of the Resolution Requirements for a DDES for a Complete High-Lift Wing}

In order to be able to compare the resolution for different test cases, all scales are given in percentage of the retracted chord length. In the case of the A320 the average retracted chord length in the refined region around the slat track, $c=0.253 \mathrm{~m}$, is used. The span-wise extent of the refined mesh region is $L_{y}=26 \% c$. The average cell size in the stream-wise and span-wise direction in this region is $\Delta_{x}=\Delta_{y}=0.14 \% c$. Hereby, in the focus region, the number of points in span-wise direction can be roughly approximated as $L_{y} / \Delta_{y} \approx 200$. With about $50 \times 10^{6}$ points in total in the refined region this leaves about $N_{x, z}=250,000$ points in one plane normal to the span-wise direction.

The resolution of the limited zonal DDES is extrapolated in order to estimate the expected computational effort of a global DDES for a similar high-lift wing configuration. The geometry has a span width of $L_{\text {span }}=425 \% \mathrm{c}=1.088 \mathrm{~m}$. Neglecting the span-wise variation of the chord length and using the same resolution $\Delta_{y}$ over the entire wing leads to a total number of points $N_{y}=3000$ in the span-wise direction. If in each plane a similar resolution with $N_{x, z}=250,000$ was used, the total number of points would be $750 \times 10^{6}$. In this estimation the nacelle is omitted.

Even if it were possible to avoid the geometrical difficulties described above and thus to halve the computational effort, with the estimated time step of $\Delta t=5 \times 10^{-6} \mathrm{~s}$ (focus on aerodynamics) an overall real time of about 10 years would be necessary to simulate $50 t_{C T U}$ on the same number of processors. However, this estimate is based on a grid with a very high resolution of the slat cove area, as in the zonal approach the focus was on the acoustics in this region. If the local phenomena of the high-lift devices are not resolved in particular and instead the focus of a hybrid RANS/LES simulation is on the wake of the entire airfoil, then the costs can be reduced considerably. In order to investigate the physics of the high-lift devices, the need for a test case with manageable complexity became obvious. The remedy was using a cut through a wing with deployed high-lift devices with periodic boundary

Kroll et al. (Eds.): Computational Flight Testing, NNFM 123, pp. 43-57, Springer, 2013 
conditions in the span-wise direction. This way the size of the computational domain can be decreased considerably and it is possible to focus on the local flow phenomena that are caused by the high-lift devices.

\section{DLR-F15 Three-Element Airfoil: Test Case Description}

The investigated DLR-F15 three-element airfoil was developed in the project LEISA as a two-dimensional cut through a generic aircraft wing with deployed slat and flap [7]. Here, experimental data at a moderate angle of attack of $\alpha=7^{\circ}$ are used, as the separation at the wind-tunnel side-walls is considered minor for low angles. Thus the flow can be regarded as almost two-dimensional and it is justified to use periodic boundary conditions in the span-wise direction in the simulations. Measurements were performed in the low-speed wind-tunnel NWB at DLR Braunschweig at a Reynolds number of $R e=2 \times 10^{6}$ based on the retracted chord length and a Mach number of $M a=0.15$. The wind-tunnel model has a chord length of $c=0.6 \mathrm{~m}$ and a span width of $2.8 \mathrm{~m}$. The experimental data used for validation consist of the mean pressure distribution in three span-wise sections and infrared measurements to locate the transition from laminar to turbulent flow. However, there are no experimental data available for the mean velocity or the Reynolds stresses.

Even though the geometrical complexity of the test case is significantly reduced, compared to the full-aircraft configuration, the accurate prediction of the physical phenomena of the flow is still very challenging: All elements produce a wake flow that interacts with the following elements. Additionally, driven by an adverse pressure gradient, the flow separates at the flap at this angle of attack. In order to capture this separation the upstream flow must be accurately predicted.

In a three-dimensional simulation the geometrical extent of the computational domain is fixed, whereas the decision about the span-wise extent of the quasi twodimensional simulation is left to the user. Here, some guidelines can be found in [8], where the coherence of the perturbation pressure over the span-wise extent is analyzed. The authors write that the span-wise extent of the computational domain should be at least $80 \%$ of the slat chord length $c_{\text {slat }}$ in order to allow the solution in the slat cove and on the main wing element nose to become completely de-correlated if periodic boundary conditions are used. Here, with $c_{\text {slat }}=20 \% c$ this results in a recommended span-wise extent of $16 \% c$. On the other hand using twice the height of the separation region on the flap (2.5\%c in the RANS simulations) is considered sufficient for the resolution of the separation. In the presented simulations a spanwise extent of $9 \% c$ is chosen, to ensure sufficient resolution of the separation on the flap.

The geometrical quality of the grid elements can be easily controlled in this case: The three-dimensional grid is obtained by stacking a number of identical two-dimensional grids with constant step size in the span-wise direction (y-direction). The twodimensional grid has also been constructed with CENTAUR and consists of about 200,000 points in the xz-plane with 2000 points on the surfaces of all three elements and 45 cells normal to the wall in the structured layers. The first node above solid walls is located at a distance of about one in plus units. The cell size in the focus region, i.e. the slat cove and in the separation region above the flap, is about $0.14 \% c$.

Kroll et al. (Eds.): Computational Flight Testing, NNFM 123, pp. 43-57, Springer, 2013 
Using 64 layers of this grid in span-wise direction over a spanwise extent of $9 \% \mathrm{c}$ leads to a total number of $12.8 \times 10^{6}$ points. After the first DDES simulation the grid was redesigned. The number of points was not significantly changed but the resolution in the slat cove and above the flap was increased, while the resolution of the leading edges of all elements was decreased. With $\ell=0.6 \mathrm{~m}$ and a free-stream velocity of $u_{\infty}=50 \mathrm{~m} / \mathrm{s}$ one convective time unit is $t_{C T U}=0.012 \mathrm{~s}$; resolving one CTU by 600 steps the time step in the simulation was chosen as $\Delta t=2 \times 10^{-5} \mathrm{~s}$.

Tab. 1: Temporal and spatial resolution of the A320 and the DLR-F15 test case

\begin{tabular}{|l|r|r|}
\hline & A320 & DLR-F15 \\
\hline \hline cell size in focus region & $0.0014 c$ & $0.0014 c$ \\
\hline No. of points on surface & 2600 & 2000 \\
\hline No. of wall-normal structured points & 30 & 45 \\
\hline No. of points in xz-plane & 250,000 & 200,000 \\
\hline time step $\Delta t$ & $1 \times 10^{-5} \mathrm{~s}$ & $2 \times 10^{-5} \mathrm{~s}$ \\
\hline No. of time steps per $t_{C T U}$ & 370 & 600 \\
\hline
\end{tabular}

In Table 1 the spatial and temporal resolution of the A320 and the DLR-F15 test case are compared.

\section{DLR-F15 Three-Element Airfoil: Results}

Part of the following simulations were performed within the European project ATAAC [9] and a detailed presentation is given in [10]. Here only those results are repeated that are relevant to the current investigations. Three different approaches were applied: a DDES, a zonal DDES and an IDDES. Concerning the second question raised in the introduction regarding the best approach to treat multi-element airfoils the results can give a guideline: The DDES approach seems to be unable to handle the confluence of the shear layer of the slat with the boundary layer on the main-wing element. Two possibilities to handle this flaw were exploited:

1. In the confluent region the RANS mode is enforced and the transport of instabilities into the boundary layer is suppressed.

2. IDDES is used which was designed to deal with unsteadiness in boundary layers by switching into an wall modeled LES mode. Therefore, it should be an ideal candidate for the interaction between a shear layer and an underlying boundary layer, sufficient resolution in space and time provided.

In the following, snapshots of the flow are compared for the different hybrid approaches. Then the mean pressure distributions are compared with RANS results.

Vorticity An instantaneous snapshot of the contour of the vorticity is shown in Fig. 3. This snapshot gives an impression of the large range of scales that are present in the flow.

Kroll et al. (Eds.): Computational Flight Testing, NNFM 123, pp. 43-57, Springer, 2013 

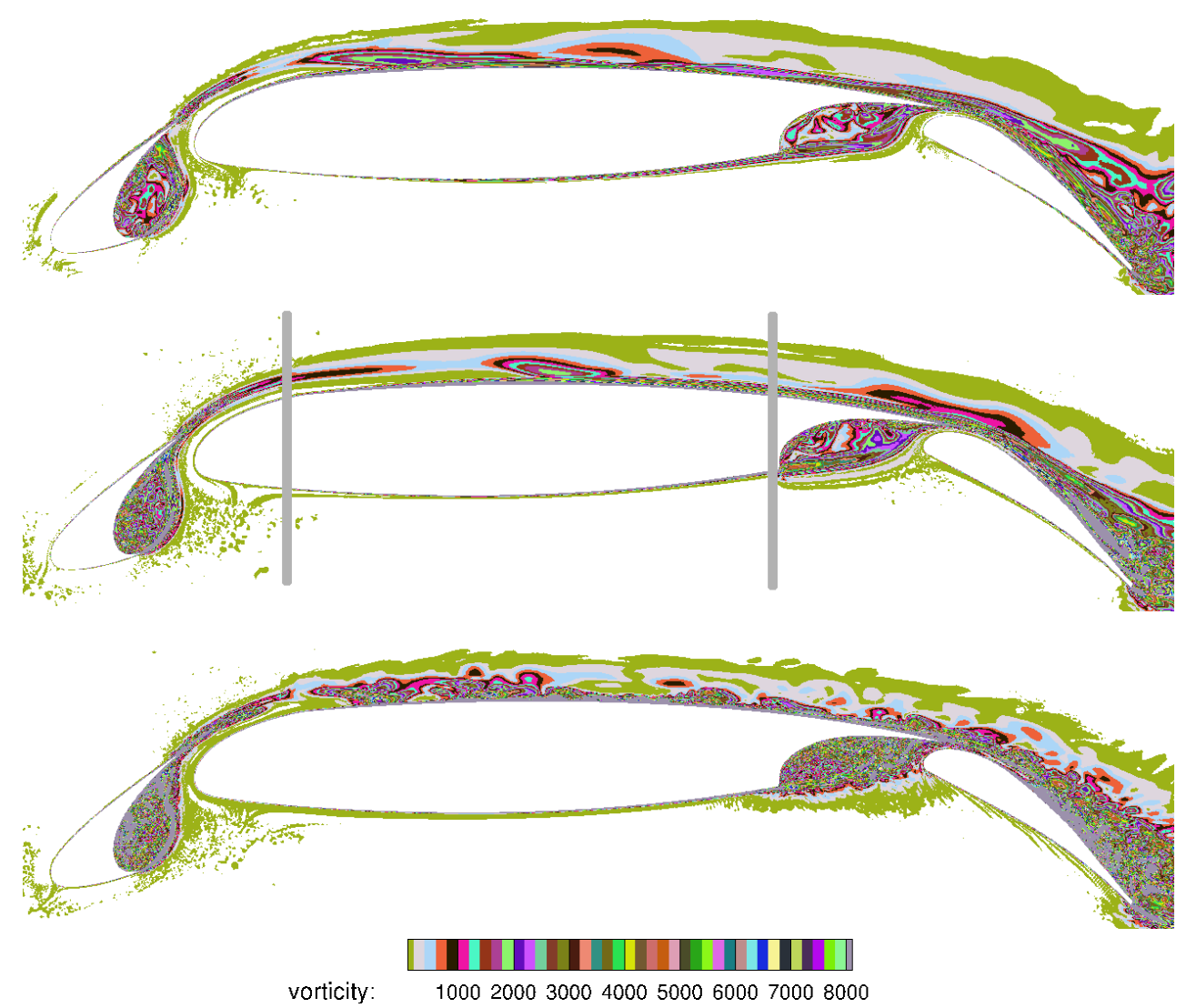

Fig. 3: Instantaneous contour of the vorticity: SA-DDES (top), zonal SA-DDES (middle) and SA-IDDES (bottom).

The vortical structures in the DDES (top) are quite coarse in the slat cove. Behind the slat trailing edge the free shear layer breaks up and the unsteady structures penetrate into the boundary layer on the main wing. This disturbance of the formal RANS layer is persistent all the way over the main wing element even very close to the wall, where a steady-state RANS solution would be expected. Associated with these unsteady events, additional turbulent kinetic energy is transported into the boundary layer and at the same time it seems that the turbulence production of the RANS model is increased, leading to massively overpredicted levels of turbulent shear stress. This shear stress is responsible for the wall-normal transport of momentum in the boundary layer. An increased shear stress also leads to a higher transport of momentum towards the wall over the flap, which prevents the separation on the flap.

In the vorticity distribution of the zonal DDES (middle), the boundaries of the enforced RANS region are indicated by the gray lines. The redesigned grid has a positive effect on the resolved structures in the slat cove. Even though the situation is formally the same as in the non-zonal DDES up to the interface of the enforced RANS zone, the structures are much smaller than before. Once the free shear layer enters the RANS region, the vortical structures are not damped immediately. Even though the shear layer is preserved longer compared to the non-zonal DDES, it breaks

Kroll et al. (Eds.): Computational Flight Testing, NNFM 123, pp. 43-57, Springer, 2013 
up finally and again penetrates into the near wall region of the boundary layer, leading to a similar situation as in the standard DDES case on the less resolved grid.

For the IDDES (bottom) the vorticity contours show again a break up of the shear layer behind the slat. The resolved structures in the slat cove as well as above the flap are much smaller than in the zonal approach. Compared to the DDES result the vortical structures that travel along the main wing element are much smaller and they are preserved all the way to the trailing edge, indicating that the IDDES runs in WMLES mode.

Pressure Distribution The pressure distributions of all three hybrid approaches are compared with two different Spalart-Allmaras based RANS simulations in Fig. 4. These reference RANS simulations differ in the way how the transition from laminar to turbulent flow is handled. In the first case, RANS 1 , the transition is prescribed on all elements, i.e. the slat is laminar, on the main wing and flap transition locations are prescribed similar to the observed position in the infra-red measurements. In the second simulation, RANS 2, no laminar regions are prescribed on the flap. This choice has a big influence on the solution and uncertainties concerning the transition settings are transported to the hybrid simulations since it is not feasible to compare different settings for all the approaches. Therefore, the setting without laminar regions on the flap was chosen. The DDES was carried out for about $22 t_{C T U}$. With

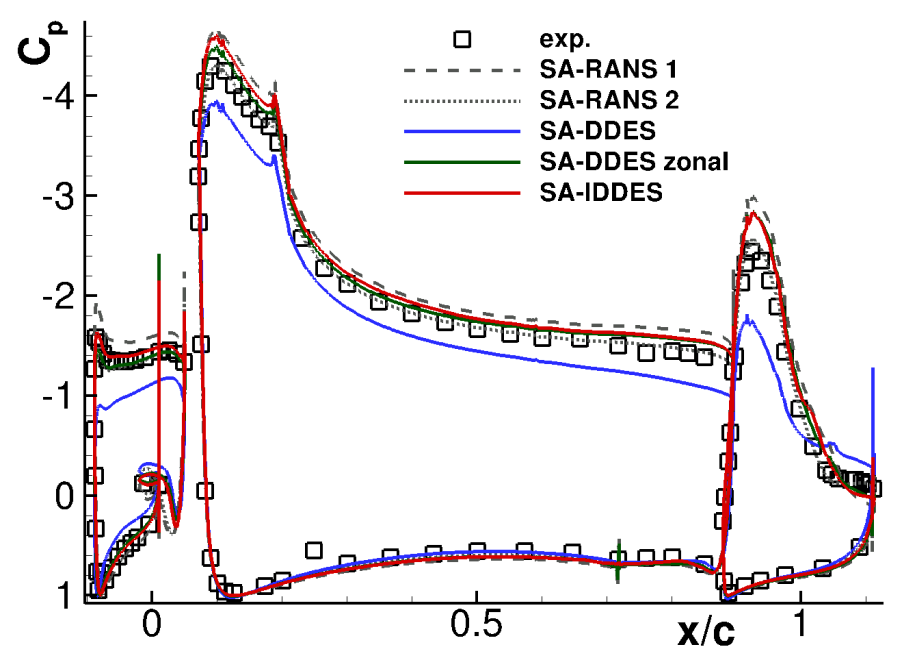

Fig. 4: Mean pressure distribution from SA RANS, DDES, zonal DDES and IDDES

this approach the flow stayed attached: in the pressure distribution no plateau can be seen at the rear of the flap. The suction peaks on all elements are too small and the pressure distribution deviates significantly from the experimental data and the RANS simulations.

In a zonal DDES the RANS mode was enforced around the main wing element. This simulation ran for about $30 t_{C T U}$ and then was stopped when again no separation on the flap was observed. The pressure distribution is in better agreement with RANS and experiments than the non-zonal DDES. The missing separation on the flap leads to higher suction peaks on the slat and main wing and the plateau on the flap is not

Kroll et al. (Eds.): Computational Flight Testing, NNFM 123, pp. 43-57, Springer, 2013 
reproduced.

The IDDES was run for the longest time until statistical convergence was reached after about $100 t_{C T U}$. The mean and RMS values were evaluated based on the last $40 t_{C T U}$. The suction peaks in the pressure distribution are higher than in the zonal DDES simulation but a small plateau can be seen at the rear of the flap. The IDDES approach predicts separated flow on the flap.

\section{DLR-F15 Three-Element Airfoil: Assessment of Resolution}

The grid resolution was estimated using three different approaches. An additional RANS simulation using the Wilcox $k \omega$-model was performed. The turbulent length scale from that RANS simulation was used to evaluate the resolution in the recirculation region and in the free shear layer. A global estimate of the resolution was obtained from the comparison of the modeled and the resolved turbulent kinetic energy. The ratio of modeled to total shear stress was used to evaluate the resolution in the near wall regions.

\subsection{Turbulent Length Scale}

In Fig. 5 , the turbulent length scale $\ell=\sqrt{k} / \omega$ that is computed from the $k \omega$-RANS simulation is shown as percentage of the chord length. The length scale in the slat cove is about $\ell=0.07 \% c$ and in the shear layer behind the slat even $\ell=0.04 \% c$. With a cell size of $0.14 \% c$ in the grid the resolution is of the same order of magnitude, but little too low. With the approach of generating the three-dimensional grid by stacking two-dimensional grids in span-wise direction, a higher resolution does not seem feasible, given the computational ressources.

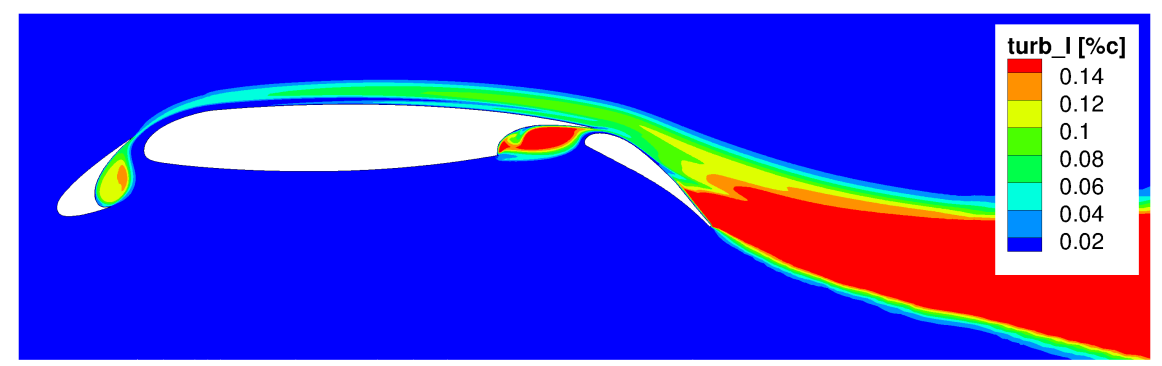

Fig. 5: Turbulent length scale $\ell$ from $k \omega$-RANS as percentage of the chord length

\subsection{Ratio of Modeled to Resolved Turbulent Kinetic Energy (TKE)}

In Fig. 6, the resolved TKE that is obtained in the IDDES simulation is compared with the modeled TKE predicted with the $k \omega$-RANS simulation. Above the flap the

Kroll et al. (Eds.): Computational Flight Testing, NNFM 123, pp. 43-57, Springer, 2013 


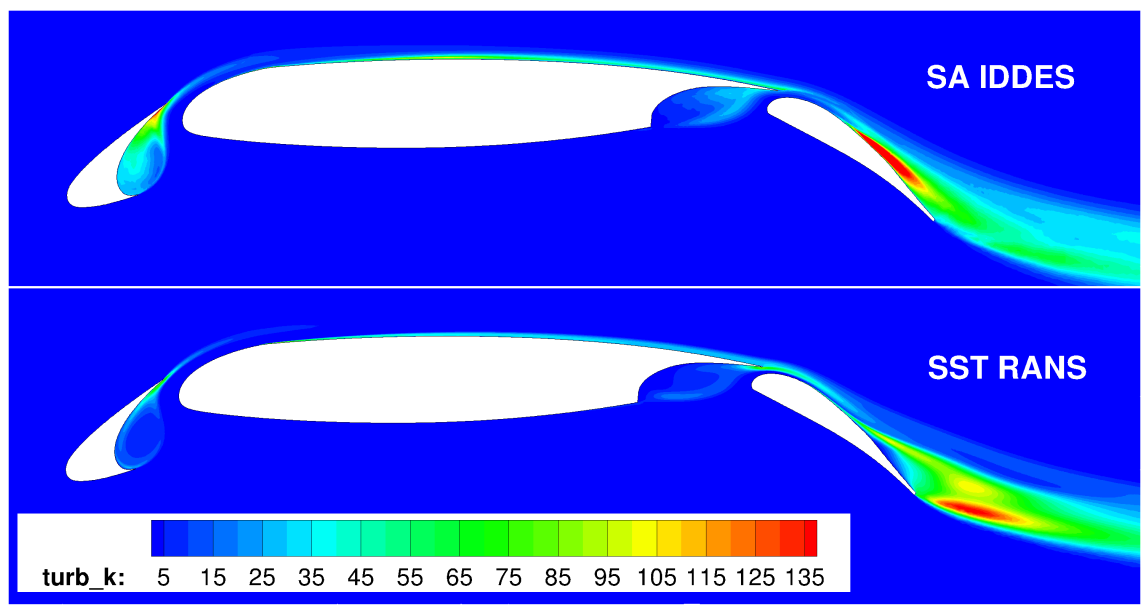

Fig. 6: Turbulent kinetic energy (in $\mathrm{m}^{2} / \mathrm{s}^{2}$ ) from SA-IDDES (top) and from $k \omega$-RANS (bottom)

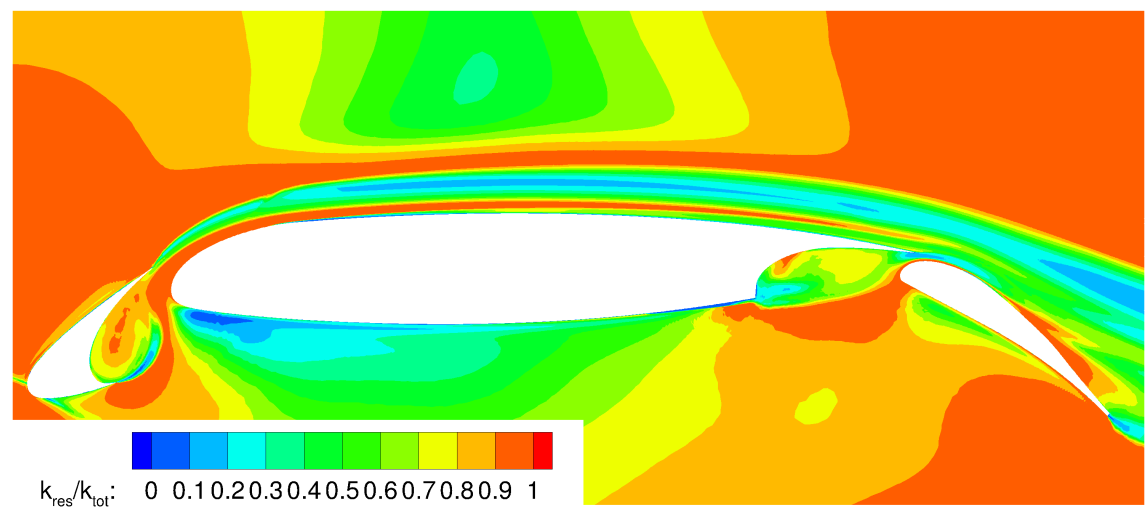

Fig. 7: Ratio of resolved TKE to total TKE in the SA-IDDES

level of resolved energy is of the same order of magnitude in the two approaches. However, in the slat and wing coves the resolved TKE is higher in the IDDES. In Fig. 7 the ratio of the modeled to the total turbulent kinetic energy is shown. In the literature a value of 0.8 is taken as a threshold for a well resolved LES. This value is reached in the slat cove; however, in the wake behind the slat this estimation suggests that the wake is under-resolved.

\subsection{Ratio of Modeled to Resolved Shear Stress}

The total turbulent shear stress is given by

$$
\left(\tau_{x z}\right)_{\text {total }}^{\text {turb }}=\underbrace{\mu_{t}\left(\frac{d u}{d z}+\frac{d w}{d x}\right)}_{\left(\tau_{x z}\right)_{\text {modelled }}^{\text {turb }}}-\underbrace{\rho\left\langle u^{\prime} w^{\prime}\right\rangle}_{\left(\tau_{x z}\right)_{\text {resolved }}^{\text {turb }}} .
$$

Kroll et al. (Eds.): Computational Flight Testing, NNFM 123, pp. 43-57, Springer, 2013 
In Fig. 8 the ratio of the modeled to the resolved shear stress in the IDDES on the upper side of the main wing element is shown. The thick solid line indicates the edge of the boundary layer, estimated by the $\Xi=y|d u / d y|$ criterion of Stock and Haase [11]. The dashed line indicates a value of the hybrid switch function $f_{d}=0.95$

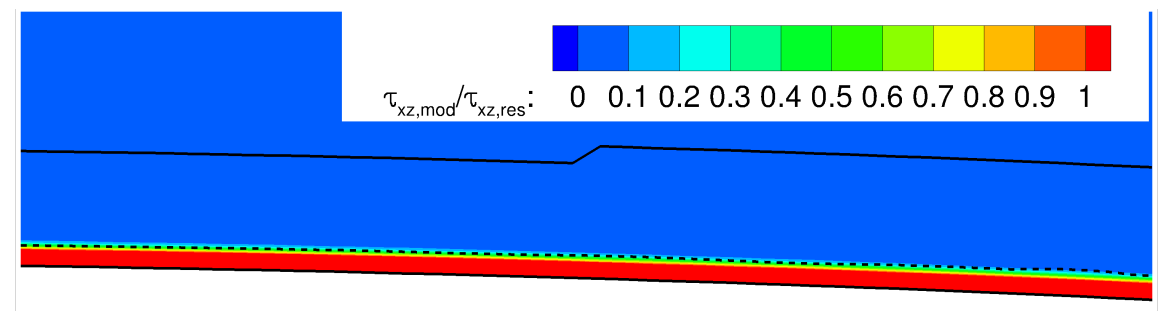

Fig. 8: Ratio of modeled to resolved shear stress on the upper side of the main wing element in the IDDES

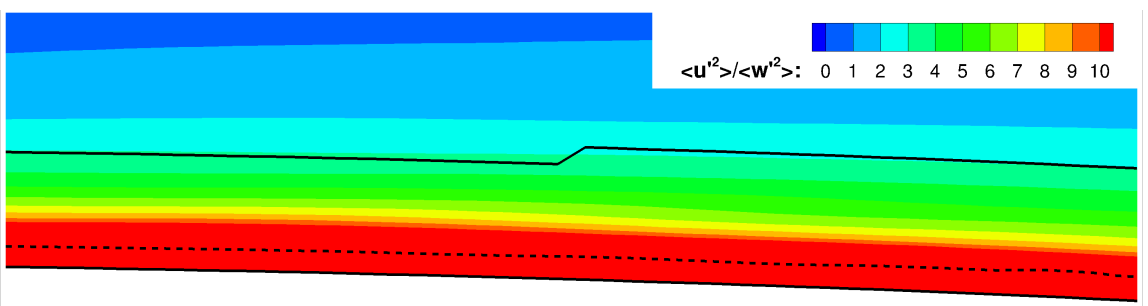

Fig. 9: Ratio of resolved velocity fluctuations in stream-wise and wall-normal direction on the upper side of the main wing element in the IDDES

and thus the edge of the formal RANS region of the hybrid approach. Here it can be seen, that the whole formal RANS region is treated in the RANS mode since the modeled stress is larger than the resolved stress. To further estimate the resolution in the outer part of the boundary layer, the ratio of the averaged velocity fluctuations in the stream-wise and the wall-normal direction is shown in Fig. 9. In an underresolved LES artificially large streaks in the stream-wise direction can be observed [12]. These structures can be identified and characterized by a large anisotropy in the ratio of the stream-wise to the wall-normal velocity fluctuations. In a well resolved LES a ratio of about 1.5 is expected [13]. Here, the ratio is about 8 in the outer part of the boundary layer indicating an under-resolved LES. Finally the resolution in the wake behind the slat trailing edge is estimated using the same approach. The ratio of the stream-wise to the wall-normal velocity fluctuations, shown in Fig. 10, takes values between 4 and 9 in a part of the wake. This indicates again that a higher resolution of the wake would be needed.

Kroll et al. (Eds.): Computational Flight Testing, NNFM 123, pp. 43-57, Springer, 2013 


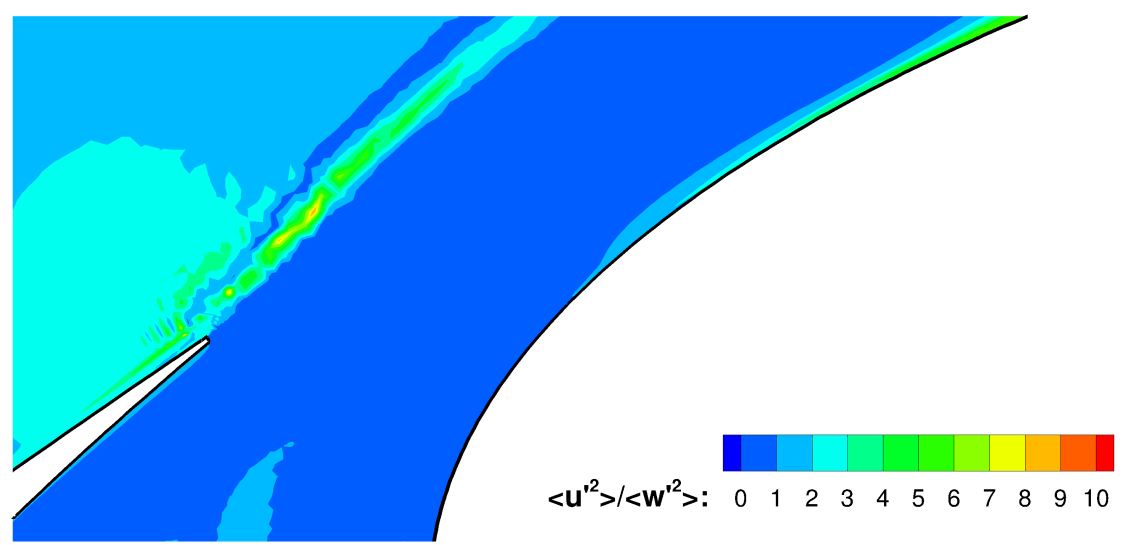

Fig. 10: Ratio of resolved velocity fluctuations in the stream-wise and the wall-normal direction in the wake behind the slat trailing edge in the IDDES

\section{Conclusions and Outlook}

Results of a zonal DDES of a A320 wing-body configuration with deployed slat and flap were presented. The focus of this simulation was on the acoustics in the slatcove. Even for this zonal approach it was not possible to achieve converged mean flow and turbulent statistics. However, conclusions regarding the computational effort of such a simulation could be drawn. The presented results were used to estimate the computational effort of a global DDES simulation for a similar configuration and it could be stated that this simulation would not be possible within the framework of the ComFliTe project.

As a consequence, the DLR-F15 three-element airfoil was chosen as the further test case and three hybrid approaches were tested: zonal DDES, global DDES and IDDES. Instantaneous snapshots of the vorticity were compared for all approaches and it was found, that the DDES is not able to handle the confluence of the free shear layer behind the slat with the attached boundary layer on the main wing. Enforcing the RANS mode in the region of confluence in the zonal approach is not sufficient, if no additional means are taken to damp the resolved structures in the free shear layer. With both approaches, zonal and global DDES, the separation on the flap was suppressed through this improper representation of the confluence through the hybrid model. One possibility would be to use a much coarser grid in the enforced RANS region in the zonal approach in order to increase the numerical dissipation and damp the LES solution. This approach could be recommended from an engineering point of view if the focus of a simulation is on local features while unsteady features in other regions can remain unresolved.

The IDDES approach, however, was able to predict the separation on the flap. This demonstrates that this approach is able to handle the interaction of the slat wake with the boundary layer on the main wing in a physically correct way. However, the evaluation of the data indicates that the flow was still under-resolved, which leads to the conclusion that the IDDES approach is very promising, but that a very highly resolved grid is needed.

Kroll et al. (Eds.): Computational Flight Testing, NNFM 123, pp. 43-57, Springer, 2013 


\section{Acknowledgment}

The authors are very grateful to their colleague S. Melber-Wilkending from the transport aircraft branch of the DLR institute of aerodynamics and flow technology for providing the basic RANS grid for the zonal DDES of the A320 and to the colleagues J. Wild and M. Pott-Pollenske for the assistance regarding the aeroacoustic questions. We also thank Airbus for funding this work and providing the experimental data for the A320 test case.

\section{References}

[1] Spalart, P. R., Jou, W. H., Strelets, M. K., Allmaras, S. R.: Comments on the feasibility of LES for wings, and on a hybrid RANS/LES approach. In 1. AFOSR Int. Conf. "Advances in DNS/LES" Aug. 4-8, In "Advances in DNS/LES", (1997)

[2] Notes on Numerical Fluid Mechanics and Multidisciplinary Design, Vol. 103: DESider - A European Effort on Hybrid RANS-LES Modelling, (2009)

[3] Schwamborn, D., Gardner, A., von Geyr, H., Krumbein, A., Lüdeke, H., Stürmer, A.: Development of the TAU-Code for aerospace applications. In 50th NAL International Conference on Aero-space Science and Technology, (2008) http://www.nal.res.in/nal50/incast/incast/01-Invited

[4] Spalart, P. R., Deck, S., Shur, M. L., Squires, K. D., Strelets, M. K., Travin, A.: A New Version of Detached-eddy Simulation, Resistant to Ambiguous Grid Densities. Theoretical and Computational Fluid Dynamics, vol. 20, no. 3, pp. 181$195,(2006)$

[5] Shur, M. L., Spalart, P. R., Strelets, M. K., Travin, A.: A hybrid RANS-LES approach with delayed-DES and wall-modelled LES capabilities. International Journal of Heat and Fluid Flow, vol. 29, no. 6, pp. 1638-1649, (2008)

[6] Sutcliffe M. and Reckzeh D. and Fischer M., HICON Aerodynamics - High Lift a aerodynamic design for the future. $25^{\text {th }}$ International Congress of the Aeronautical Sciences ICAS, (2006)

[7] Wild, J., Pott-Pollenske, M.: An integrated design approach for low noise exposing high-lift devices. AIAA Paper 2006-2834, (2006)

[8] Lockard, D. P., Choudhari, M. M.: Noise Radiation from a Leading-Edge Slat. AIAA Paper 2009-3101, (2009)

[9] http://cfd.mace.manchester.ac.uk/ATAAC/WebHome

[10] Reuß, S., Knopp, T., Schwamborn, D.: Hybrid RANS/LES simulations of a three element airfoil. Notes on Numerical Fluid Mechanics and Multidisciplinary Design, Vol. 117: Progress in Hybrid RANS-LES Modelling, (2012)

[11] Stock, H. W., Haase, W.: The Determination of Turbulent Length Scales in Algebraic Turbulence Models for Attached and Slightly Separated Flows Using Navier-Stokes Methods. AIM 19th Fluid Dynamics, Plasma Dynamics and Lasers Conference, AIAA Paper 1987-1302, (1987)

Kroll et al. (Eds.): Computational Flight Testing, NNFM 123, pp. 43-57, Springer, 2013 
[12] Piomelli, U., Balaras, E.: Wall-Layer Models for Large-Eddy Simulations. Annual Review of Fluid Mechanics, no. 34, pp. 349-374, (2002)

[13] Pope, S. B.: Turbulent Flows. Cambridge University Press, (2000)

Kroll et al. (Eds.): Computational Flight Testing, NNFM 123, pp. 43-57, Springer, 2013 


\title{
[D]
}

\section{Assessment of Local LES-Resolution Sensors for Hybrid RANS/LES Simulations}

\author{
S. Reuß, T. Knopp, A. Probst, M. Orlt \\ Institute of Aerodynamics and Flow Technology DLR (German Aerospace Center), \\ Bunsenstrasse 10, 37073 Göttingen
}

\section{Published in:}

Girimaji, S., Haase, W. Peng, S.H., Schwamborn, D. (Eds.): Progress in Hybrid RANS-LES Modelling, Notes on Numerical Fluid Mechanics and Multidisciplinary Design Vol. 130, pp. 93-103, Springer, 2015

\begin{abstract}
Different sensors that provide a measure for the resolution of the LES content in hybrid RANS/LES computations are proposed and investigated. In an apriori test on isotropic turbulence a suitable sensor is identified. Based on that sensor an automatic local mesh refinement is performed for an IDDES of the flow over a backward facing step. The results obtained on locally adapted grids are compared to results on globally refined grids. It is shown, that the proposed sensors can detect underresolved LES regions and that the local mesh refinement can help to reduce resolution errors caused by a too coarse grid spacing.
\end{abstract}

\section{Introduction}

The assessment of the grid resolution in hybrid RANS/LES methods is crucial to ensure the reliability of the results [1]. Therefore, only a very careful mesh design can provide sufficient resolution in the LES regions while keeping the total number of points as low as possible. In complex cases, where the location of the LES regions is not known in advance, this may even be impossible. 
Thus, robust sensors are proposed in this work to assess the LES resolution. They are applied in combination with the automatic grid adaptation module, that is provided by the DLR-TAU code [12].

The paper is organized as follows: In Sect. 2 the numerical method that is used throughout all simulations and the adaptation algorithm are described. Specific details about the resolution sensors are given in Sect. 3 and the proposed sensors are assessed in a-priori tests on isotropic turbulence. Actual mesh refinements based on one of the proposed sensors are performed for the flow over a backward facing step in Sect. 4. In Sect. 5 conclusions are drawn and an outlook is given.

\section{Numerical Method}

The simulations are performed using the DLR TAU code, an unstructured finite volume solver for the compressible Navier-Stokes equations, that provides hybrid RANS/LES methods based on different RANS models. The influence of the numerical settings on the quality of scale resolving simulations is subject to an in-depth study in [11]. The reference settings that are described there are used in this work.

\section{The Hybrid RANS/LES Method}

All simulations that are shown here use the Improved Delayed DES (IDDES) [13] based on the Menter-SST RANS model. In [4] it was shown, that a modification of the filter width in the hybrid model can help to reduce the grey area in Zonal DES. Following the same route here, the filter width in the IDDES is modified, using $\Delta_{\omega}=\sqrt{N_{x}^{2} \Delta_{y} \Delta_{z}+N_{y}^{2} \Delta_{z} \Delta_{x}+N_{z}^{2} \Delta_{x} \Delta_{y}}$, with $\boldsymbol{N}$ the normalized vorticity vector. In the present unstructured dual grid metric $\Delta_{x}$ is the maximal extent in $\mathrm{x}$-direction of all connected dual-edges. In the IDDES the original length scale comprises three different parts: the maximum length $h_{\max }$ and the maximum wall normal extent $h_{w n}$ of all connected dual edges and the wall distance $d_{w}$ : $\Delta=\min \left(\max \left[C_{w} d_{w} ; C_{w} h_{\max } ; h_{w n}\right] ; h_{\max }\right)$. The maximum edge length $h_{\max }$ also occurs in $\alpha=0.25-d_{w} / h_{\max }$. In the modified filter width $h_{\max }$ is replaced by $\Delta_{\omega}$, however, only the second occurrence in the length scale equation is replaced, in order to preserve the wall-modelled LES capabilities. In [11] this procedure is validated for the fully-developed channel flow.

\section{The Adaptation Algorithm in the DLR-TAU Code}

The TAU-adaptation module [2] was originally designed for the equidistribution of differences or gradients of a preliminary flow solution by local hierarchical grid re- and derefinement. The main steps of the adaptation algorithm include a preparing loop over all elements. Considering the geometry and the implemented subdivision cases of the element type, some of the edges are excluded from subdivision. This check is repeated until all edges that would lead to invalid refinement cases are forbidden.

In the next step all edges allowed for subdivision that are marked by the edge indicator are subdivided. A following loop over all elements checks and ensures the validity of all element refinements by subdivision of additional edges and faces if needed. In

Girimaji et al. (Eds.): Progress in Hybrid RANS-LES Modelling, NNFM 130, pp. 93-103, Springer, 2015 


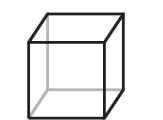

regular subdivision

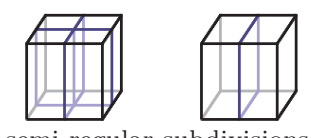

semi-regular subdivisions

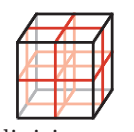

西

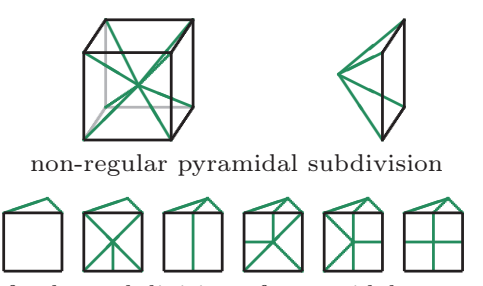

further subdivision of pyramidal parts
Fig. 1: Regular (left) and irregular bridging (right) hexahedral subdivision cases.

this step all the bridging subdivisions between the refined and unrefined grid areas are created.

The new point coordinates at the surface are determined by a quadratic spline interpolation to reconstruct the curvature. In the inner grid parts a linear interpolation is used. Finally, the new elements are constructed and the current solution is interpolated to the new points. The result is a locally refined grid with bridging elements introduced into the original grid structure. In Fig. 1 the implemented subdivisions for hexahedral elements are shown.

\section{Resolution-Sensors for Local Mesh Adaptation}

In this work the original adaptation indicator is replaced by a suitable sensor that attempts to measure the quality of the LES resolution. Several sensors have been proposed in the literature and some of them are presented here.

Since a grid refinement in hybrid RANS/LES simulations not only reduces the numerical error but also changes the model, in [7] and [3] systematic grid and model variations have been performed to distinguish the numerical from the modelling error. For complex test cases these exhaustive variations are not affordable. Instead, a resolution estimation that can be obtained from a single simulation is preferable. One concept for such a sensor, proposed by Pope [10], is the ratio of the resolved turbulent kinetic energy (TKE) $k_{r e s}$ to the total TKE $k_{t o t}$. In practice the total TKE is split into the resolved grid scale and the modelled subgrid-scale (sgs) TKE:

$$
S=\frac{k_{r e s}}{k_{\text {total }}}=\frac{k_{\text {res }}}{k_{\text {res }}+k_{\text {sgs }}} .
$$

The resolved TKE can be computed directly from the resolved quantities, $k_{\text {res }}=$ $1 / 2\left\langle(\boldsymbol{u}-\langle\boldsymbol{u}\rangle)^{2}\right\rangle$, where $\boldsymbol{u}$ is the velocity and $\langle\boldsymbol{u}\rangle$ denotes the mean. But for the subgrid-scale TKE an appropriate model is required. In [8] it is proposed to estimate the sgs TKE contribution by explicitly filtering the solution:

$$
k_{s g s}=\frac{1}{2}\left\langle\boldsymbol{u}_{s g s}^{2}\right\rangle
$$

where $\boldsymbol{u}_{s g s}=\boldsymbol{u}-\langle\overline{\boldsymbol{u}}\rangle$. The space-filtered velocity $\overline{\boldsymbol{u}}$ is given by the convolution integral $\overline{\boldsymbol{u}}=\int_{\Omega_{1}} \mathcal{G}(\boldsymbol{x}-\boldsymbol{y}) \boldsymbol{u}(\boldsymbol{y}, t) \mathrm{d} \boldsymbol{y}$ where $\mathcal{G}$ denotes the top hat filter function with filtering kernel $\Omega_{1}$. In the following the filtering is denoted as $\overline{\boldsymbol{u}}=\mathcal{G} * \boldsymbol{u}$. In [8] the kernel of the top hat filter comprises all direct neighbours, whereas here larger supports of the filter function are tested, too. A filter with a larger support is constructed by recursive application of the top hat filter: $\mathcal{G}_{\Omega n} * \boldsymbol{u}=\mathcal{G}^{n} * \boldsymbol{u}=$ $\mathcal{G} * \ldots * \mathcal{G} * \boldsymbol{u}$.

Girimaji et al. (Eds.): Progress in Hybrid RANS-LES Modelling, NNFM 130, pp. 93-103, Springer, 2015 
Using these extended filters the approximate deconvolution model (ADM) [14], [5] provides another possibility to compute $\boldsymbol{u}_{s g s}$. Here, the unfiltered velocity is constructed using the approximated inverse of a filter function. The idea is to use a filter $\mathcal{G}$ with a filter width larger than the local mesh spacing. The filtered solution $\boldsymbol{u}_{2}=\mathcal{G} * \boldsymbol{u}$ contains only scales larger than the smallest scales that can be resolved on the grid. The solution $\boldsymbol{u}_{1}$ that would be obtained using the local mesh spacing as filter width is reconstructed by the deconvolution of the coarser solution: $\boldsymbol{u}_{1}=\mathcal{G}^{-1} * \boldsymbol{u}_{2}$. This approach is used in order to get an estimation of the exact solution as follows: Let $\boldsymbol{u}_{\mathrm{DNS}}$ be the solution of a DNS and $\mathcal{G}$ a filter operator, then the solution of an LES is $\boldsymbol{u}_{\mathrm{LES}}=\mathcal{G} * \boldsymbol{u}_{\mathrm{DNS}}$ or $\boldsymbol{u}_{\mathrm{DNS}}=\mathcal{G}^{-1} * \boldsymbol{u}_{\mathrm{LES}}$. If $H=I d-\mathcal{G}$ is replaced into the geometric series

$$
\sum_{j=0}^{\infty} H^{j}=1 /(1+H)=(1+H)^{-1} \text { then follows } \mathcal{G}^{-1}=\sum_{j=0}^{\infty}(1-\mathcal{G})^{j}
$$

Now the series representation is truncated at a finite $N$. In [14] is stated that $N=3$ already yields acceptable results, and for $N>5$ no further improvement can be expected, so $N=4$ is chosen here. This gives

$$
\mathcal{G}^{-1}=5-10 \mathcal{G}+10 \mathcal{G}^{2}-5 \mathcal{G}^{3}+\mathcal{G}^{4}
$$

With the decomposition $\boldsymbol{u}_{\mathrm{DNS}}=\boldsymbol{u}_{\mathrm{LES}}+\boldsymbol{u}_{\text {sgs }}$ the sgs velocity is given by

$$
\boldsymbol{u}_{s g s}=\left(I d-\mathcal{G}^{-1}\right) * \boldsymbol{u}_{\mathrm{LES}}
$$

Another model for the subgrid-scale TKE is proposed by Lilly [9]:

$$
k_{\text {sgs }}=\left\langle\nu_{t}\right\rangle^{2} /(c \Delta)^{2}, \text { with } c=0.094
$$

If the basic RANS model provides a model-equation for the TKE (e.g. $k-\omega, k-\varepsilon$ ), it is also possible to use the mean of that quantity:

$$
k_{s g s}=\langle k\rangle \text {. }
$$

\section{Validation of the Sensors Based on Isotropic Homogeneous Turbulence}

For an a-priori assessment of the different sensor formulations, they are evaluated for the initial field of the well known decaying isotropic turbulence (DIT) test case. The computational domain is a cubic volume with an edge length $L=2 \pi$. The number of points in the fine, medium and coarse grid $G 1, G 2$ and $G 3$ are $n_{1}=128^{3}, n_{2}=64^{3}$ and $n_{3}=32^{3}$ respectively. For the DIT measured spectra are available, from which experimental values for the sensors can be estimated. The cut-off wave-number is $\kappa_{c}=n_{p n t} / 2$. The total TKE is the area under the power spectrum $k_{t o t}=\int_{0}^{\infty} E(\kappa) \mathrm{d} \kappa$ while the resolved TKE is $k_{\text {res }}=\int_{0}^{\kappa_{c}} E(\kappa) \mathrm{d} \kappa$.

The initial solution is constructed as a superposition of all frequencies from the measured spectrum. From this initial solution the different sensor formulations are evaluated. In this test the homogeneous turbulence is regarded as frozen and the temporal average is replaced by a spacial average. Thus an a-priori evaluation is possible. In Fig. 2 the resulting sensor values are listed. On the $x$-axis the relative cell size w.r.t. to the finest grid cell size is plotted.

Girimaji et al. (Eds.): Progress in Hybrid RANS-LES Modelling, NNFM 130, pp. 93-103, Springer, 2015 


\begin{tabular}{llllll}
\hline & Eq. for $k_{\text {sgs }}$ & $G 1$ & $G 2$ & $G 3$ & \\
\hline Exp. & - & 0.92 & 0.75 & 0.55 & - \\
$S_{1}, n=2$ & $(2)$ & 0.83 & 0.78 & 0.71 & --- \\
$S_{1}, n=4$ & $(2)$ & 0.77 & 0.71 & 0.64 & - \\
$S_{2}$ & $(3)$ & 0.58 & 0.47 & 0.37 & - \\
$S_{3}$ & $(4)$ & 0.80 & 0.74 & 0.68 & - \\
$S_{4}$ & $(5)$ & 0.85 & 0.81 & 0.76 & - \\
\hline
\end{tabular}

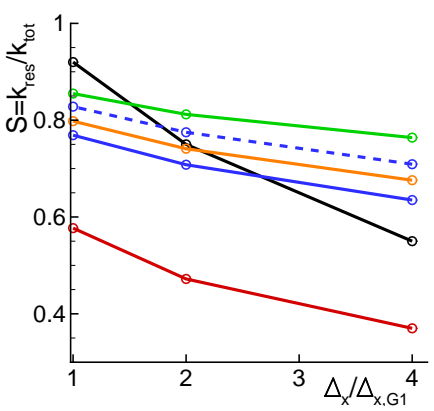

Fig. 2: A-priori sensor values for different grids for the DIT test case.

The most complex sensor $S_{2}$, which is based on the approximate deconvolution model, reproduces the slope of the experimental TKE ratio the best. However, it gives an overall far too pessimistic estimation of the grid resolution, therefore it is not chosen for the sample application to the backward facing step in Sect. 4 .

Apart from $S_{2}$, the slope of all sensors is similar except for a constant offset. They all estimate the resolution of the coarsest grid too optimistic and underestimate the resolution of the fine grid. The sensor $S_{1}$ with the smaller explicit filter size as well as the sensor $S 4$ also overestimate the medium grid.

Since an underpredicted grid resolution is considered the conservative (i.e. "safer") approach the sensor $S_{1}$ with the larger support of $n=4$ is chosen for the sample application in Sect. 4.

\section{Modified Adaptation Indicator}

The above sensor definitions provide a local value for each grid point. However, since the sensors are only applicable to LES, a certain amount of local resolved turbulence is necessary, to draw conclusions in hybrid RANS/LES computations. Therefore, the evaluation of the sensor is limited to regions where a specified threshold of the resolved turbulence intensity $T=1 / u \sqrt{2 / 3\left(\left\langle u^{\prime 2}\right\rangle+\left\langle v^{\prime 2}\right\rangle+\left\langle w^{\prime 2}\right\rangle\right)}$ is exceeded. In this work $T_{0}=0.1$ is chosen, as this value is typically reached in a turbulent boundary layer.

For the adaptation algorithm an edge-wise indicator is constructed: The edge is marked for subdivision if the sensor indicates an insufficient grid resolution at one of the adjacent points, i.e. $S<S_{0}$. Since in [10] it is stated that an LES should resolve at least $80 \%$ of the turbulence by the grid, the threshold is chosen as $S_{0}=0.8$. Additionally, the mode of the hybrid RANS/LES model is taken into account: If the model operates in RANS mode, i.e. the IDDES hybrid switching-function $\tilde{f}_{d}=1$, the edge is not refined.

A smoothing of the refined region is performed by spreading the indicator over further elements. Elements touched by the edge indicator and their neighbours up to a specified number of element layers around them are subdivided isotropically to make sure that the marked grid parts reside completely in the isotropically refined grid area. In the present work, marking four additional layers of elements leads to acceptably smooth regions.

Girimaji et al. (Eds.): Progress in Hybrid RANS-LES Modelling, NNFM 130, pp. 93-103, Springer, 2015 
Fig. 3: Dimensions and denotation of the computational domain of the flow over a backward facing step.

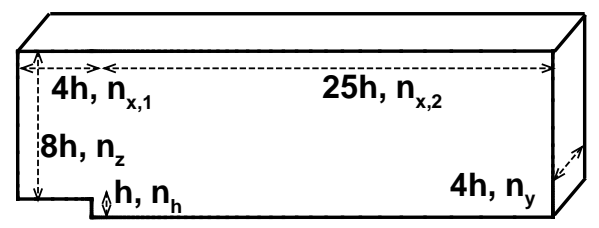

Tab. 1: Number of grid points in the globally refined and the locally adapted grids.

\begin{tabular}{rrrrrr}
\hline & $G 1$ & $G 2 A$ & $G 2$ & $G 3 A$ & $G 3$ \\
\hline$n_{\text {pnt }}$ & 2.127 .000 & 810.000 & 274.000 & 132.000 & 36.000 \\
\hline
\end{tabular}

\section{Application to a Backward Facing Step Flow}

The test case of the flow over a backward facing step (BFS) is determined in terms of the step hight $h=0.0127 \mathrm{~m}$. Experimental data for a Reynolds-number based on $h$ of $R e_{h}=37500$ are given by Driver and Seegmiller [6]. The dimensions of the computational domain are shown in Fig. 3. The point-numbers in the coarse grid $G 3$ are $n_{x, 1}=11, n_{z}=21, n_{x, 2}=50, n_{h}=24$ and $n_{y}=15$. A family of grids $G 1$ fine, $G 2$ medium and $G 3$ coarse - has been constructed based on $G 3$ by a global refinement with a factor of 2 in each direction.

Based on the sensor $S_{1}$ (cf. Fig. 2) the medium and coarse grids have been locally adapted. The adapted grids are referred to as $G 2 A$ and $G 3 A$ hereafter. Since in the adaptation algorithm the edges are bisected, the resolution in the refined regions is the same as in the next globally refined grid, i.e. $G 1$ and $G 2 A$ have the same resolution in the adapted LES region, $G 2$ and $G 3 A$ respectively. A comparison of the total point-numbers in the different grids is shown in Table 1.

First, the sensor values resulting from the different mesh resolutions are compared in Fig. 4. Regions with a local turbulence intensity lower than $T_{0}=0.1$ are blanked. In the left column the globally refined grids are shown, the respective adapted coarser grid is shown in the same line in the right column, the adapted regions are outlined by the black lines.

Here it can be seen how the sensor reacts to the mesh refinement: The values in the adapted regions are at the same level as those on the globally refined meshes.

In Fig. 5 the spanwise averaged sensor values at a position of $x / h=4$ behind the step at half the step height are compared. On the $x$-axis the relative cell size w.r.t. to the finest grid cell size is plotted. The sensor values on the globally refined grids and those on the locally adapted grids almost match exactly. All sensors indicate that in the finest grid more than $80 \%$ of the turbulence is resolved. In contrast to the a-priori test for the DIT, the sensors now show a clearer distinction. The sensors $S_{3}$ and $S_{4}$, that are directly based on $\nu_{t}$ and $k_{R A N S}$ from the background RANS model, do not react to the grid refinement, so that they are not suited as an indicator for the grid resolution in this test case. Again the values from $S_{1}$ with the two different explicit filter widths share the same slope but exhibit an offset.

Remarkably, the sensor $S_{2}$ that still shows the steepest slope now yields a positive estimation for the finest grid. One explanation might be, that the a-priori test is

Girimaji et al. (Eds.): Progress in Hybrid RANS-LES Modelling, NNFM 130, pp. 93-103, Springer, 2015 


\section{G1 - SST IDDES $\triangle$}

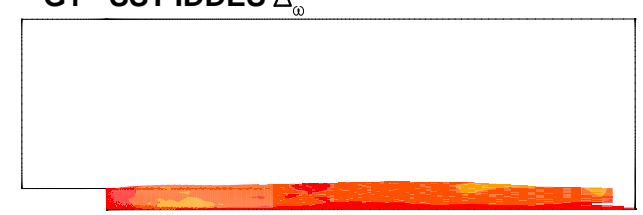

G2 - SST IDDES $\triangle$

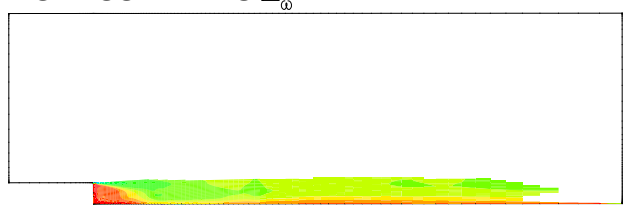

G3 - SST IDDES $\triangle$

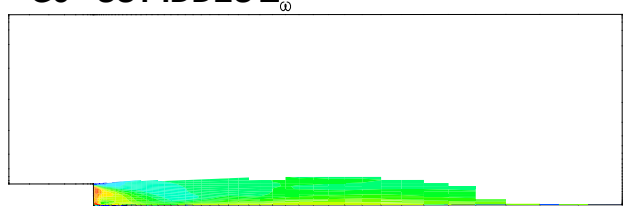

G2A - SST IDDES $\triangle$

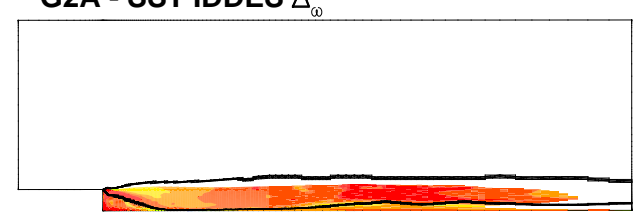

G3A - SST IDDES
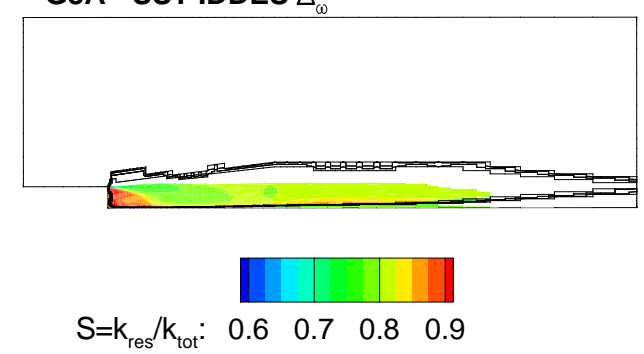

Fig. 4: Comparison of the grid sensor values on different grids. The values are blanked if the turbulence intensity is smaller than $T_{0}=0.1$. On the left the globally refined grids are shown, on the right the locally adapted grids are shown.

\begin{tabular}{lcccccc}
\hline & $G 1$ & $G 2 A$ & $G 2$ & $G 3 A$ & $G 3$ & \\
\hline \hline$S_{1}, n=2$ & 0.93 & 0.93 & 0.87 & 0.87 & 0.79 & --- \\
$S_{1}, n=4$ & 0.87 & 0.87 & 0.78 & 0.78 & 0.68 & - \\
$S_{2}$ & 0.86 & 0.87 & 0.71 & 0.72 & 0.48 & - \\
$S_{3}$ & 0.98 & 0.98 & 0.98 & 0.98 & 0.99 & - \\
$S_{4}$ & 0.95 & 0.95 & 0.94 & 0.93 & 0.94 & - \\
\hline
\end{tabular}

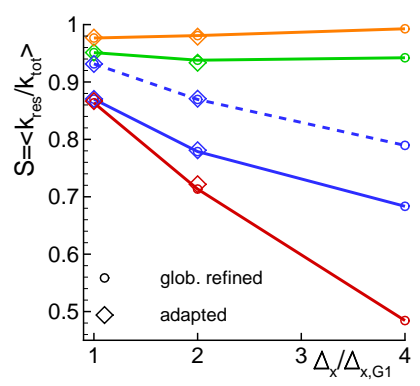

Fig. 5: Sensor values for different grids for the BFS test case.

not suited for this sensor, because it does not only depend on the subgrid velocity but also on the mean velocity itself which is zero for the DIT test case. It seems worthwhile to assess this sensor in other applications.

In Fig. 6 the flow behind the step is shown. Following the streamtraces, four regions can be distinguished, where different physical and modelling phenomena characterize the simulation. The extent of the regions depends on the numerical scheme and on the grid design. Upstream of the step, for $x / h<0$ an attached turbulent boundary layer is modelled in RANS mode, region 0 . In the first region of interest, region $I$ $(x / h=0$ to $x / h \approx 2)$ immediately behind the step, the hybrid model switches from RANS to LES, which may be delayed due to the "grey-area" problem. An important feature in region I is the topology of the secondary recirculation bubble in the corner of the step. Its size influences the primary recirculation, by pushing the centre of the primary recirculation further downstream. The second region, region $I I(x / h \approx 2$ to $x / h \approx 7)$ around the reattachment point, is dominated by the LES and characterized by high values of resolved TKE. The third region, region III $(x / h>7)$ downstream

Girimaji et al. (Eds.): Progress in Hybrid RANS-LES Modelling, NNFM 130, pp. 93-103, Springer, 2015 
G1

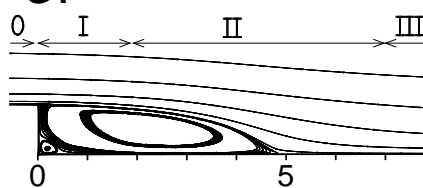

G2A

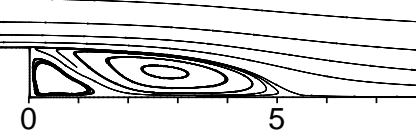

G2A

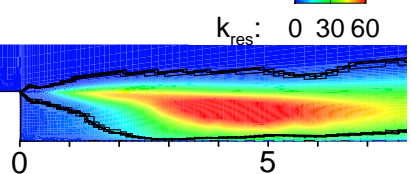

Fig. 6: Streamlines in the recirculation area in the fine (left) and adapted medium (middle) grid. Resolved turbulence on the adapted medium grid $G 2 A$ (right), the adapted region is outlined.
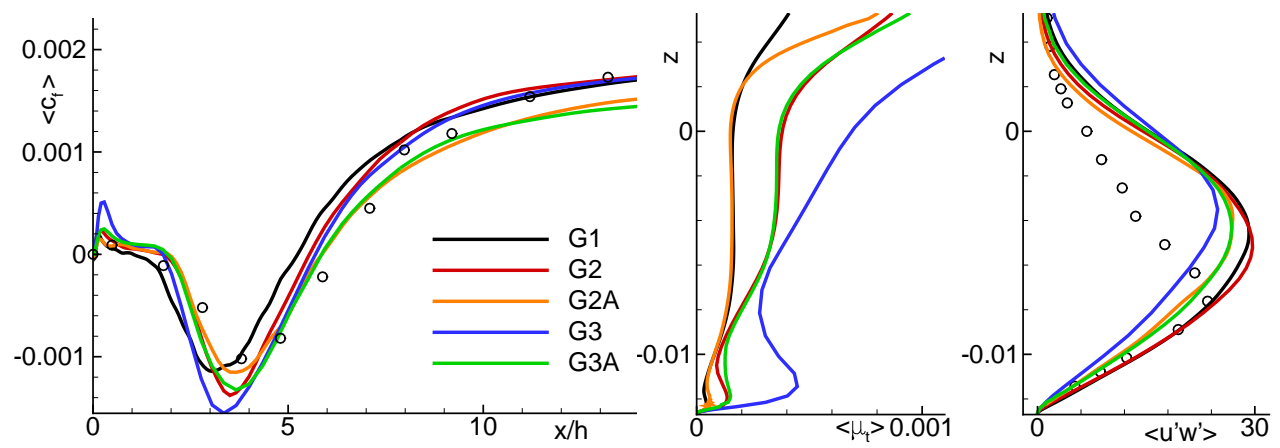

Fig. 7: Skin friction distribution (left), resolved Reynolds-stresses (middle) and eddyviscosity in a section at $x / h=4$ (right) for different grids.

of the reattachment, is essentially a resolved attached boundary layer with turbulent inflow. The different characteristics that dominate the flow make this a good testcase to examine the validity as well as the limits of the proposed adaptation strategy.

It was found that using the filter width $\Delta_{\omega}$ reduces the grey area in region I significantly compared to the $\Delta_{\text {edge }}$-filter (not shown here) and taht resolved turbulence develops quickly behind the step. However, in the grids $G 2$ and $G 3$ the resolution in the centre region is rather coarse, and due to a low level of resolved turbulence intensity this region is excluded from refinement. The coarse resolution of the upper part of the shear layer can have a negative influence on its development.

As depicted in Fig. 4 the region of the secondary flow is considered well resolved according to the sensor and thus not refined in the medium grid $G 2 A$. Nevertheless, the size of this secondary recirculation is much larger than in grid G1. Both, this and the resolution, influence the downstream behaviour of the flow. In Fig. 7 (left) the skin friction distributions for all grids are compared. The plateau at $x / h=0$ to $x / h \approx 2$ is the footprint of the secondary corner flow which has the smallest extent on the finest grid $G 1$. All other simulations are comparable in this regard.

The highest values of resolved turbulence are reached in region II. Here, the minimum of the skin friction, i.e. the strongest backflow, is obtained. The location of the minimum is influenced by the upstream flow, but in terms of the absolute minimum value the effect of the grid refinement can be compared. Through the global grid refinement the absolute values decrease. As expected, the locally adapted meshes yield almost the same minimum values.

In Fig. 7 (middle) the mean values of the eddy-viscosity $\left\langle\mu_{t}\right\rangle$ are shown for a cut at $x / h=4$. The global mesh refinement leads to lower levels of modelled turbulence.

Girimaji et al. (Eds.): Progress in Hybrid RANS-LES Modelling, NNFM 130, pp. 93-103, Springer, 2015 
The local mesh-adaptation has the same effect. The solutions from the adapted meshes, $G 3 A$ (green) and $G 2 A$ (orange), coincide with the corresponding globally refined grids, $G 2$ (red) and $G 1$ (black).

In figure 7 (right) the resolved Reynolds stresses in the $\mathrm{x}-\mathrm{z}$ plane $\left\langle u^{\prime} w^{\prime}\right\rangle$ are shown. Compared to the experimental data all of the simulations exhibit large deviations. But also when comparing the simulations among each other, no clear trend is found. The profiles on the medium grid $G 2$ agrees well with the fine grid $G 1$. However, due to the upstream flow the cut corresponds to different positions in the recirculation region. The solution on the locally adapted grid $G 2 A$ has a larger offset from the globally refined solution than from the coarse-grid solution. Also for the coarser grids the clear trend, that was observed before, is not confirmed in the Reynolds stress. Even though the local mesh adaptation moves the result in the direction of the globally refined grid, there is still a difference between the two results.

For the skin friction distribution behind the reattachment point, the local mesh adaptation has even an adverse effect on the results. While for all non-adapted refined grids the experimental skin friction is captured quite accurately, the local mesh adaptation leads to a larger offset from the experiments. This might be because the mesh refinement ends at the near-wall RANS layer. The strategy to confine the adaptation indicator to LES regions seems not suited for attached turbulent boundary layers in wall-modelled LES mode. Instead, it may be necessary to extend the refined region down to the wall in this case.

\section{Conclusion}

Different formulations of a mesh sensor that assesses the resolution in the LES region have been investigated. In the case of isotropic turbulence the proposed sensors have been compared to experimental values. One of the sensors has been evaluated for a family of grids for a backward facing step and was used as indicator for a local mesh adaptation. The results of the globally and locally refined grids have been compared, and the range of validity as well as the limits of the sensor have been examined.

It has been shown, that the proposed sensors are able to detect underresolved regions in an LES. Moreover, the sensors can be used as input for a local mesh refinement. This is an important step to automatically reduce the uncertainty w.r.t. mesh design in hybrid RANS/LES modelling.

In the future the sensors will be applied to more complex testcases. In the case of wall-modelled LES the possibility to improve the results by extending the refined region down to the wall will be assessed.

\section{Acknowledgement}

The authors gratefully acknowledge the "Deutsche Forschungsgemeinschaft" (German Research Foundation) who funded this work in the scope of the Research Group DFG FOR 1066. \%

Girimaji et al. (Eds.): Progress in Hybrid RANS-LES Modelling, NNFM 130, pp. 93-103, Springer, 2015 


\section{References}

[1] Yacine Addad, Ulka Gaitonde, and Stefano Laurence, Dominique Rolfo. Optimal Unstructured Meshing for Large Eddy Simulations. In Johan Meyers, Bernard J. Geurts, and Pierre Sagaut, editors, Quality and Reliability of LargeEddy Simulations, Ercoftac Series, pages 93-103. Springer Netherlands, 2008.

[2] Thomas Alrutz and Matthias Orlt. Parallel dynamic grid refinement for industrial applications. In proceedings of ECCOMAS CFD 2006, Wesseling, P. Oñate, E. Périaux, J. (eds.), The Netherlands, TU Delft, 2006.

[3] I. B. Celik, Z. N. Cehreli, and I. Yavuz. Index of Resolution Quality for Large Eddy Simulations. Journal of Fluids Engineering, 127(5):949, 2005.

[4] Nicolas Chauvet, Sébastien Deck, and Laurent Jacquin. Zonal Detached Eddy Simulation of a Controlled Propulsive Jet. AIAA Journal, 45(10):2458-2473, October 2007.

[5] F. K. Chow, R. L. Street, Ming Xue, and J. H. Ferziger. Explicit Filtering and Reconstruction Turbulence Modeling for Large-Eddy Simulation of Neutral Boundary Layer Flow. Journal of Atmospheric sciences, 62:2058-2077, 2005.

[6] David M. Driver, H. Lee Seegmiller, and Joe G. Marvin. Time-dependent behavior of a reattaching shear layer. AIAA Journal, 25(7):914-919, July 1987.

[7] M. Klein. An Attempt to Assess the Quality of Large Eddy Simulations in the Context of Implicit Filtering. Flow, Turbulence and Combustion, 75(1-4):131147, December 2005.

[8] Tobias Knopp, Xiaoqin Zhang, Roland Kessler, and Gert Lube. Enhancement of an industrial finite-volume code for large-eddy-type simulation of incompressible high Reynolds number flow using near-wall modelling. Computer Methods in Applied Mechanics and Engineering, 199(13-16):890-902, February 2010.

[9] Douglas K Lilly. The Representation of Small-Scale Turbulence in Numerical Simulation Experiments. NCAR Manuskript No. 281, (November), 1966.

[10] S. B. Pope. Turbulent Flows. Cambridge University Press, 2000.

[11] Axel Probst and Silvia Reuß . Scale-resolving simulations of wall-bounded flows with an unstructured compressible flow solver. In 5th Symposium on Hybrid RANS-LES Methods TEXAS A\&M University, College Station, Houston, USA, 19-21 March 2014, 2014.

[12] Dieter Schwamborn, Thomas Gerhold, and Ralf Heinrich. The DLR TAU-Code: recent applications in reaserach and industry. In proceedings of ECCOMAS CFD 2006, Wesseling, P. Oñate, E. Périaux, J. (eds.), The Netherlands, TU Delft, 2006.

[13] Mikhail L. Shur, Philippe R. Spalart, Mikhail Kh. Strelets, and Andrey K. Travin. A hybrid RANS-LES approach with delayed-DES and wall-modelled LES capabilities. International Journal of Heat and Fluid Flow, 29(6):16381649, December 2008.

Girimaji et al. (Eds.): Progress in Hybrid RANS-LES Modelling, NNFM 130, pp. 93-103, Springer, 2015 
[14] S. Stolz, N. A. Adams, and L. Kleiser. An approximate deconvolution model for large-eddy simulation with application to incompressible wall-bounded flows. Physics of Fluids, 13(4):997, 2001.

Girimaji et al. (Eds.): Progress in Hybrid RANS-LES Modelling, NNFM 130, pp. 93-103, Springer, 2015 

[E]

\title{
Numerical investigation of the DLR F15 two-element airfoil using a Reynolds stress model
}

\author{
S. Reuß, A. Probst, T. Knopp
}

Deutsches Zentrum für Luft- und Raumfahrt, Göttingen, Germany

\author{
Presented at: \\ Joint Symposium "Simulation of Wing and Nacelle Stall", Jun. 21-22, \\ 2012, Braunschweig, Germany \\ Published online: \\ https://sfb880.tu-braunschweig.de/classtree/global/download.php? cmp=FOR1066\&f= \\ Veranstaltungen@FOR_Veranstaltungen_2012@FOR_2012_Modelling-2\&d=8-Reuss-2012.pdf
}

\begin{abstract}
This work is dedicated to the appropriate representation of the experimental setup in the numerical prediction of the flow around multi-element airfoils. In the simulations a high-fidelity Reynolds-stress RANS-model is applied. The well established Menter-SST RANS-model is used as a reference. To assess the influence of the wind-tunnel walls three different numerical approaches with different computational complexity are compared: A three-dimensional simulation of the entire measurement section, a two-dimensional cut through the centerline of the test section and the same two-dimensional cut but with inviscid wind tunnel walls. The results show that it is sufficient to restrict the simulation to the centerline section of the wind tunnel and treat the wind-tunnel walls as inviscid walls.
\end{abstract}

\section{Nomenclature}

JHh Jakirlic-Hanjalic-homogeneous

RSM Reynolds-stress model

c retracted chord length [m]

$\alpha \quad$ angle of attack [ $\left.{ }^{\circ}\right]$

$C_{l}$ non-dimensional lift-coefficient

$\mu_{t} \quad$ eddy viscosity $[\mathrm{Pa} \cdot \mathrm{s}]$ 
$k \quad$ turbulent kinetic energy $\left[\mathrm{m}^{2} / \mathrm{s}^{2}\right]$

$d_{w}^{+} \quad$ wall distance in wall-units

\section{Introduction}

One of the most important goals in the numerical prediction of aerodynamical flows is to improve the prediction of the maximum lift for multi-element airfoils. Regarding the flow physics modeling, one major challenge is the correct prediction of the free shear-layers behind upstream elements and their interaction with attached boundary layers on downstream elements under the influence of an adverse pressure gradient. The application of standard RANS-models does not yield always satisfying results for these test cases. Therefore, the high-fidelity JHh-v2 RSM [1] is used in this work. The mid term goal is to assess the possible advantages of hybrid RANS/LES simulations over pure RANS simulations. For a scale resolving simulation a very high resolution is needed. For this reason it is not feasible to model the entire experimental setup and it is desirable to restrict the numerical simulation to the centerline section. Therefore, it is important to investigate the differences between the experimental and numerical approaches. These investigations are performed using RANS simulations.

In this work three different approaches are compared: In the computationally most expensive approach the entire experimental section of measurements with resolved boundary layers on the wind tunnel walls as well as the three dimensional wind tunnel model is included in the simulation. In the second approach a two-dimensional cut through the centerline section of the wind tunnel is modeled and the influence of the side wall effects is neglected. In the third approach the upper and lower tunnel walls are treated as inviscid walls and the boundary layers at these walls are not resolved. This is the computationally least expensive and, therefore, the most desirable approach if scale resolving hybrid RANS/LES models are to be applied.

The investigated DLR F15 two-element airfoil is an industrially relevant test case in the framework of the DFG Forschergruppe 1066 (FOR-1066). The experimental database for this test case is provided by other partners in the same project [2]. To reduce the wind tunnel side wall effects in the experimental setup, the model is equipped with droop noses at the model-wall junctions. The drooped leading edge of the main wing reduces the effective incidence angle and thus the separation at the model side wall junction.

\section{Numerical method}

The numerical simulations are performed with the DLR TAU code [3], a finite volume solver for the Navier-Stokes equations on unstructured meshes. The time-accurate simulations use a dual time-step scheme with an LUSGS solver. The spacial discretization uses central differences with artificial dissipation of matrix type. The turbulence equations are discretized using a second order Roe-scheme. The convergence is accelerated by a $3 \mathrm{w}$ multi-grid scheme. Different turbulence models are implemented for the closure of the Reynolds-averaged NS-equations.

In the present paper, the two-equation model Menter k- $\omega$ SST [4] and the JHh-v2 Reynolds-stress model [1] are applied. The latter is an extended and recalibrated

In Joint Symposium "Simulation of Wing and Nacelle Stall", Jun. 21-22, 2012, Braunschweig, Germany, 2012 
variant of the JHh RSM [5] which applies a length-scale equation based on the homogenous dissipation rate $\varepsilon^{h}$ as well as low-Reynolds damping functions to accurately model near-wall turbulence.

Grids. All grids were generated using the commercial grid-generation software Gridgen which provides the generation of structured as well as unstructured regions.

The desired strategy in the envisaged hybrid RANS/LES simulation is to regard only the centerline section of the experimental setup. This approach is chosen in the two-dimensional simulations. The first strategy is to use a cut through the centerline of the test section without resolving the boundary layers of the upper and lower wind tunnel walls and instead use Euler boundary conditions. For this approach two grids are compared. The first grid 1 has a reasonable RANS resolution with about 84,000 points. About 60 hexahedral elements resolve the boundary layer in the wall normal direction with a first wall point at a distance of $d_{w}^{+} \approx 1$ in wall-units. The main wing and flap are discretized with 430 and 240 points respectively. A detail is shown in Fig. 1 (upper left). To check the grid influence, a second grid with a much finer resolution is used. This very highly resolved grid would be appropriate for a hybrid RANS/LES simulation. This grid 2 has about 200,000 points in the $\mathrm{x}-\mathrm{z}$ plane. About 100 hexahedral elements resolve the boundary layers in the wall normal direction. The first node above the wall is located at a distance of about $d_{w}^{+} \approx 0.4$ in wall-units. The surface of the main wing element is discretized with 685 points and 480 points are located on the surface of flap.

In the second approach the effects of the upper and lower wind tunnel walls are taken into account. Grid 1 was modified to resolve the boundary layers at these walls with about 60 hexahedral cells. The grid is shown in 1 (lower left).

The three-dimensional grid is based on the modified grid 1 as the centerline section. The surface grid is shown in Fig. 1 (right). The grid encloses one half of the experimental setup and uses a symmetry boundary condition at the centerline section. It contains 7.5 million grid points.

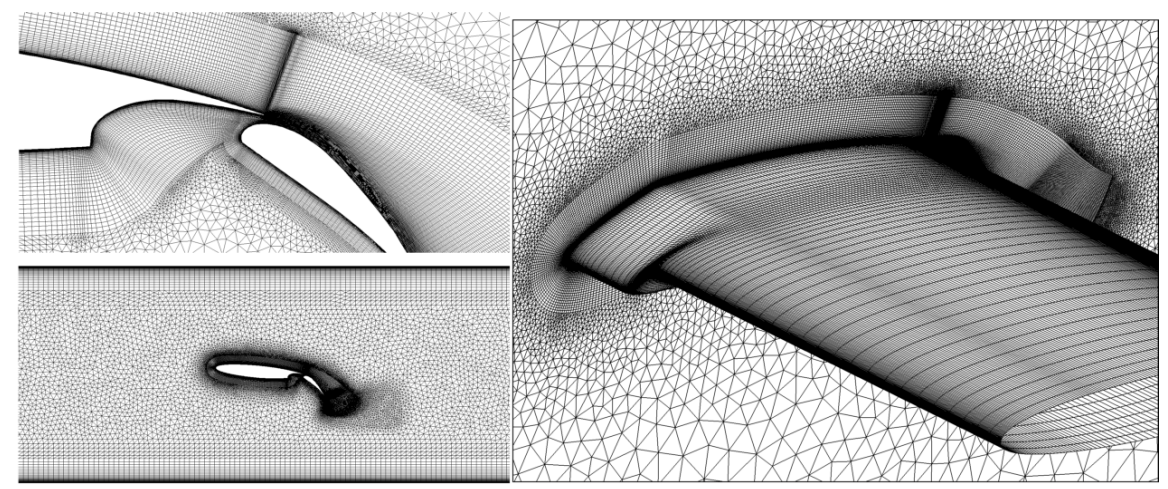

Fig. 1: Two- and three-dimensional grids used in the presented simulations

In Joint Symposium "Simulation of Wing and Nacelle Stall", Jun. 21-22, 2012, Braunschweig, Germany, 2012 


\section{Test case description}

The experimental setup consists of the measurement section of the wind tunnel and the measured F15 two-element profile. The wind tunnel has a quadratic inlet section with an edge length of $1.3 \mathrm{~m}$. The entire test section has a length of $7.55 \mathrm{~m}$. Over a length of $5.7 \mathrm{~m}$ the upper and lower wall diverge. Over the last $1.85 \mathrm{~m}$ the upper and lower wall are retracted again. In this last section the side walls are also retracted. The profile has a retracted chord length of $c=0.6 \mathrm{~m}$. The span-wise extend of the wind tunnel model is $1.3 \mathrm{~m}$. The model is positioned $3.9 \mathrm{~m}$ behind the inlet section at a centered position. The flap is deployed with a deflection angle of $\alpha_{\text {flap }}=35^{\circ}$. Detailed experimental data including static pressure measurements at the centerline section of the model and the upper and lower wall of the wind tunnel, PIV data and oil-flow pictures were taken at incidence angles of $\alpha=0^{\circ}$ and $\alpha=6^{\circ}$. These two incidence angles are used in the following simulations. The measurements were performed with a velocity of $50 \mathrm{~m} / \mathrm{s}$ at a Reynolds number of about 2 million and a Mach number of about 0.15 .

\section{Results}

In this section the results that were obtained with the different strategies described above are compared. In the first subsection the computationally least expensive results that were obtained using a two-dimensional cut in the centerline section and inviscid boundary conditions for the upper and lower wind tunnel wall are compared. This relatively inexpensive approach is used to investigate the influence of the grid resolution and the time-step size. Also a principal difference between the solutions of the two applied turbulence models, the Menter-SST and the JHh-v2 RSM model, is observed. The reason for this difference is investigated. In the next subsection the results that were obtained in the simulations that take into account the wind tunnel walls are presented and compared to the previously described results and to the experimental data.

\subsection{D simulations with inviscid upper and lower wind tunnel wall}

First the appropriate mesh resolution and time-step size are investigated. In Fig. 2 three time-accurate simulations using the Menter-SST model at an incidence angle of $\alpha=6^{\circ}$ are compared. The mean-pressure distribution, averaged in time, obtained with different grids and time-steps are shown. The green line is the pressure distribution on the coarser grid 1 while the red line is computed on the finer grid 2. There is hardly any difference between the two results and thus it is justified to use the coarser grid in the following simulations. The influence of the time-step can be seen in the comparison of the green line and the blue line. In the former simulation a physical time-step of $\Delta t=6 \cdot 10^{-5} \mathrm{~s}$ is used. This corresponds to 200 time-steps per convective time unit. The blue line is from a simulation with a physical time-step of $\Delta t=3 \cdot 10^{-5} \mathrm{~s}$, corresponding to 400 time-steps per convective time unit. The only difference between these two lines can be seen at the very rear end of the flap in the zoomed perspective. This difference is due to the resolution of the instabilities

In Joint Symposium "Simulation of Wing and Nacelle Stall", Jun. 21-22, 2012,

Braunschweig, Germany, 2012 


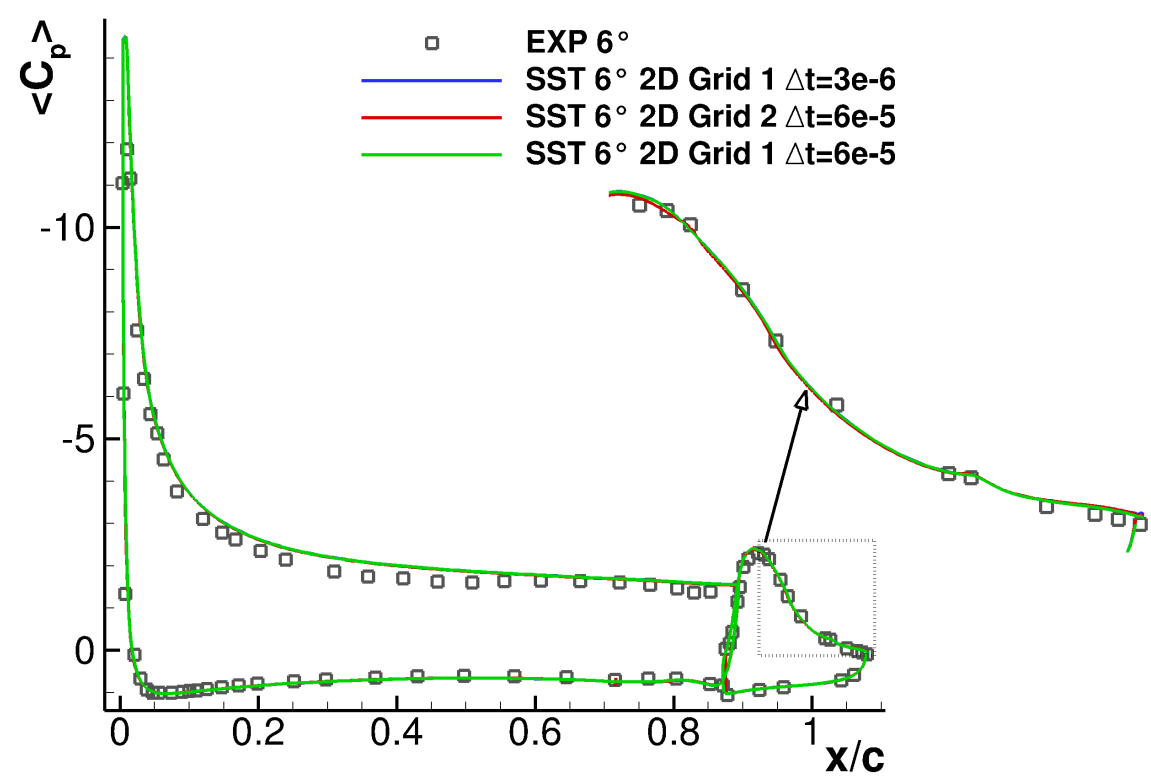

Fig. 2: Pressure distribution with Menter-SST model on grid 1 with different time steps and on grid 2

that form behind the blunt trailing edge of the flap and has no influence on the overall flow. Thus it is justified to use a time-step of $\Delta t=3 \cdot 10^{-5} \mathrm{~s}$ in the following simulations.

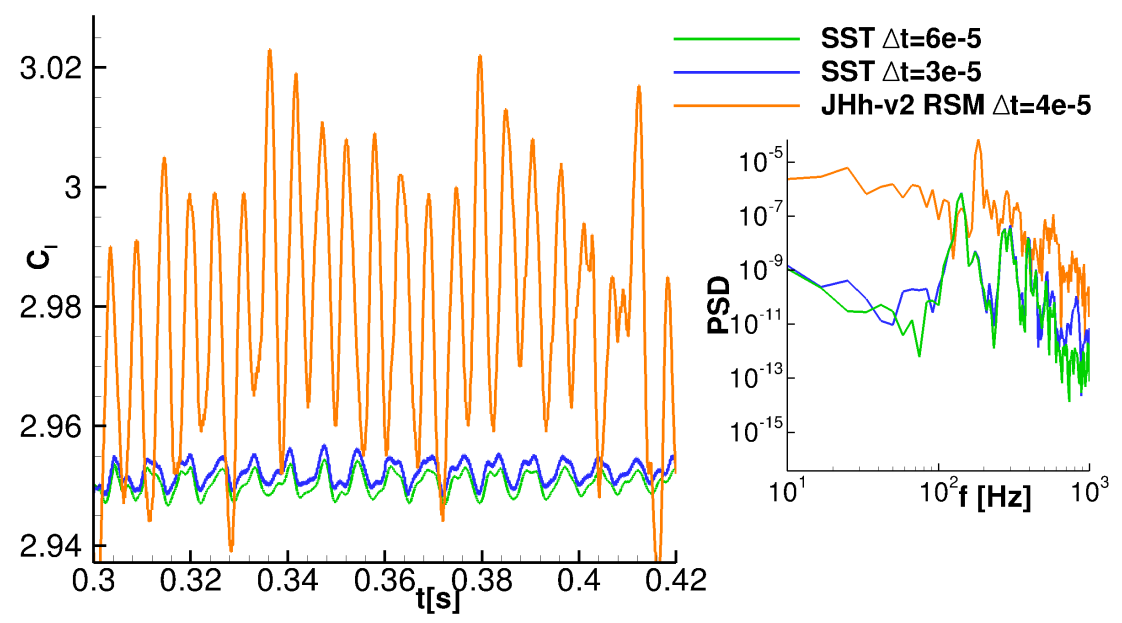

Fig. 3: Time curve of the lift-coefficient from Menter-SST and JHh-v2 RSM simulations and corresponding sound-pressure-level

This finding is confirmed by the time curve of the lift-coefficient that is shown in Fig. 3. The green and blue lines are the results discussed earlier. The main behavior is the same in both simulations. There is a very small difference of the mean lift that is $C_{l}=2.95$ with a time-step of $\Delta t=6 \cdot 10^{-5}$ s and $C_{l}=2.952$ with a time-step of $\Delta t=3 \cdot 10^{-5} \mathrm{~s}$. This little difference is again due to small differences in the very rear

In Joint Symposium "Simulation of Wing and Nacelle Stall", Jun. 21-22, 2012, Braunschweig, Germany, 2012 
part of the flap and can be neglected. The distinct peak in the sound-pressure-level at a frequency of $144 \mathrm{~Hz}$ shows up in both simulations. With the smaller time-step the level of the larger frequencies is higher. The orange line in Fig. 3 is from a JHh-v2 RSM simulation on the coarser grid. Here the lift-curve oscillates with comparatively high amplitudes with a peak frequency of $185 \mathrm{~Hz}$ and the mean lift is $C_{l}=2.979$.

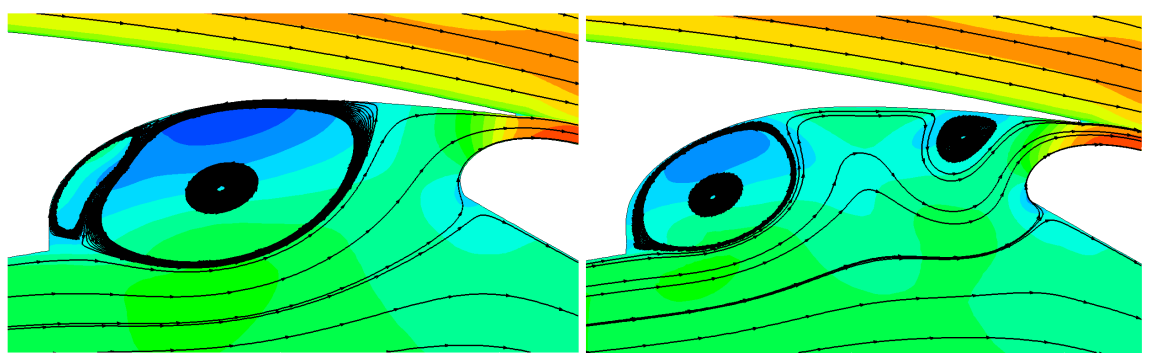

Fig. 4: Instantaneous streamlines in the wing cove at $\alpha=0^{\circ}$ predicted with the JHh-v2 RSM model

The time-accurate Menter-SST simulations converge to a nearly steady state. This is not the case for the JHh-v2 RSM model. The flow in the cove of the main wing is highly unsteady. The instantaneous streamlines in the cove at two different instances in time are shown in Fig. 4. The separation bubble blows up and then breaks down again, splitting into two smaller bubbles.
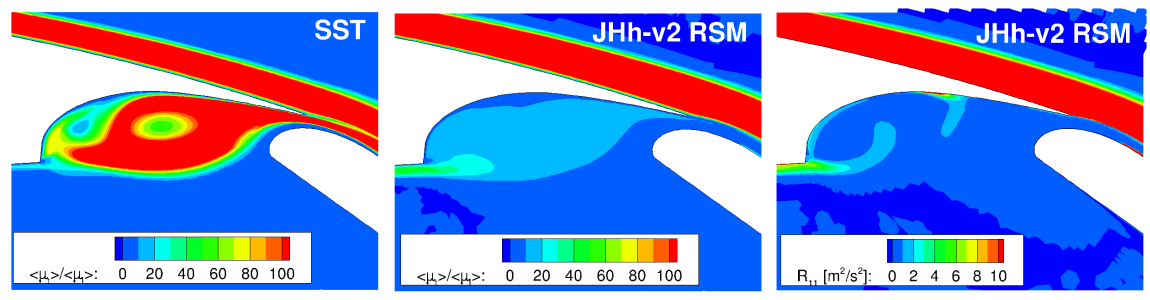

Fig. 5: Eddy-viscosity of the Menter-SST (left) and the JHh-v2 RSM simulation (middle) and Reynolds-stress $R_{11}$ (right)

The reason for this instability is shown in Fig. 5. Here the ratio of the modeled turbulent eddy-viscosity to the laminar eddy-viscosity is shown. In the RSM simulation the modeled turbulent eddy-viscosity is computed as $\mu_{t}=0.09 \cdot \rho \cdot k_{R S M}^{2} / \varepsilon$, with $k_{R S M}=0.5 \cdot R_{i i}$ with the normal Reynolds-stresses $R_{i i}$. The level of modeled turbulence in the wing cove is considerably smaller in the JHh-v2 RSM simulation. Here the $\alpha=0^{\circ}$ case is shown but the same observation can be made for $\alpha=6^{\circ}$.

This low level of modeled turbulence in the RSM simulation is due to a very quick decay of the modeled Reynolds-stresses right behind the geometry-fixed separation point at the sharp edge. The distribution of the first normal component of the Reynolds-stress tensor is shown on the right in Fig. 5. The same rapid decay of modeled Reynolds-stress occurs for all other components.

In Fig. 6 the mean-streamlines, averaged in time, obtained in the PIV measurement, with the Menter-SST model and with the JHh-v2 RSM model are compared for the incidence angle of $\alpha=0^{\circ}$. The Menter-SST model predicts the separation on the flap in good agreement with the PIV data but the onset of the separation is predicted a little too early. The influence of the instability in the RSM simulation triggers some

In Joint Symposium "Simulation of Wing and Nacelle Stall", Jun. 21-22, 2012, Braunschweig, Germany, 2012 

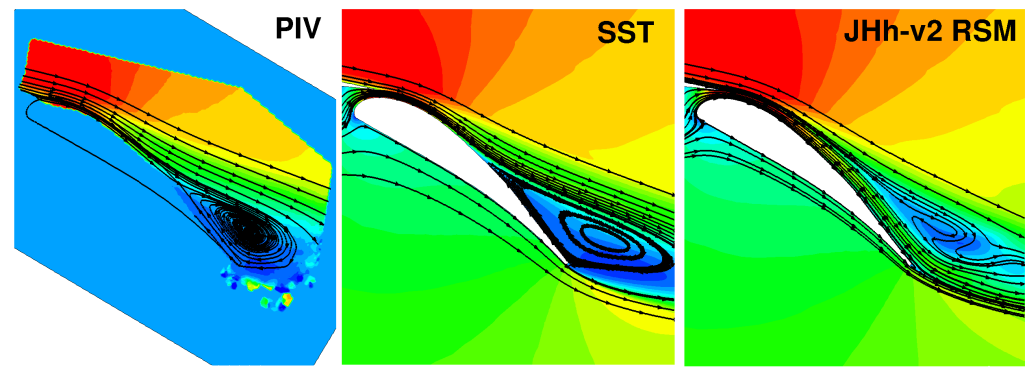

Fig. 6: Mean-streamlines, averaged in time, from experiments and with the Menter-SST and JHh-v2 RSM model at $\alpha=0^{\circ}$ colored with the stream-wise velocity

mechanism that prevents the flow from separating at the flap. Instead an instability forms between the flow over the main wing and the flow through the gap between main wing and flap. This instability leaves a footprint in the mean-streamlines.
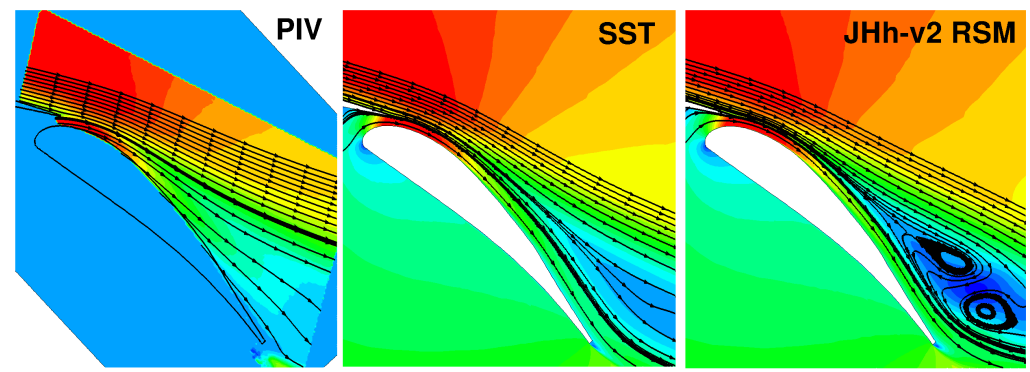

Fig. 7: Mean-streamlines, averaged in time, from experiments and with the Menter-SST and JHh-v2 RSM model at $\alpha=6^{\circ}$ colored with the stream-wise velocity

The corresponding comparison for the $\alpha=6^{\circ}$ case is shown in Fig. 7. At this incidence angle the flow on the flap is attached. This behavior is predicted by both models but there is a general difference between experiment and simulations: In the experiment the flow through the gap thickens towards the trailing edge of the flap while no strong bending is observed in the streamlines of the flow over the main wing. In both simulations the streamlines from the main wing wake are bent towards the flap while the gap flow is parallel to the flap without much thickening. In the RSM results again an instability forms in the shear layer between the flow over the main wing and the gap flow which can also be seen in the time averaged streamlines.

\subsection{Simulations with viscous wind tunnel walls}

The most expensive approach in terms of computational resources is to include the whole measurement section in the numerical setup. Results from this approach predicted by the Menter-SST model and the JHh-v2 RSM model are shown in Fig. 8. Here, the skin-friction lines are compared with oil-flow pictures from the experiment at an angle of attack of $\alpha=6^{\circ}$.

In the upper row a view of the leading edge of the main wing from upstream is depicted and the lower row presents a view of the flap from downstream. The oilflow visualization reveals a small separation region at the junction between main

In Joint Symposium "Simulation of Wing and Nacelle Stall", Jun. 21-22, 2012, Braunschweig, Germany, 2012 


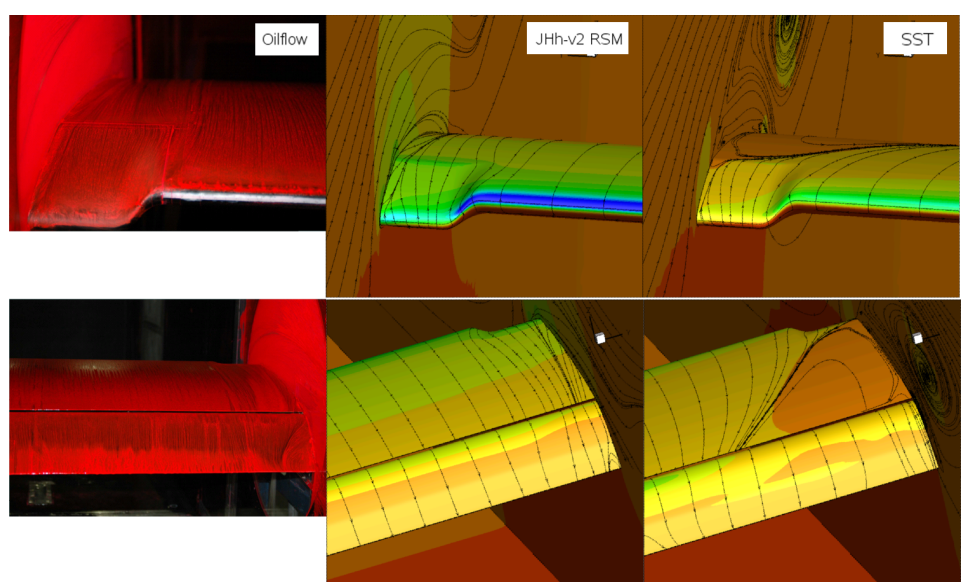

Fig. 8: Oil-flow pictures and skin-friction lines from Menter-SST and JHh-v2 RSM model at $\alpha=6^{\circ}$

wing and side-wall. The flow is bent at the smooth transition region between the droop nose and the main profile but at a small distance towards the centerline section it is essentially undisturbed. The JHh-v2 RSM model that is shown in the center column predicts this separation region at the main wing in good agreement with the experimental observations. Opposed to that in the Menter-SST result the size of the separation is massively over-predicted. In the span-wise direction the flow is influenced by this separation over a great part of the main wing towards the centerline section.

The view of the flap shows again the large influence of the separation region in the SST simulation. The massively separated flow on the main wing shields the flow on the flap such that the separation at the junction between flap and wind tunnel wall is completely missing. Again the flow pattern is predicted much better by the RSM model even though the size of the separation at the junction between flap and tunnel wall is under-predicted.

Another observation in the three-dimensional Menter-SST simulation is an unphysical separation at the centerline section which is not in agreement with the PIV data. This seems to be due to the massively disturbed flow in the centerline section because of the over-predicted side-wall separation since in the two-dimensional simulations no separation was predicted at this incidence angle.

For the $\alpha=0^{\circ}$ case only Menter-SST results are available, so far. At this lower angle of attack, however, the SST model predicts the separation at the side wall reasonably well and the flow in the centerline direction is undisturbed by the sidewall separation. Also the size of the separation at the junction between the flap and the wind tunnel side-wall is predicted in good agreement with the oil-flow picture.

The influences of the different approaches on the mean-pressure distribution in the centerline section of the wind tunnel can be seen in Fig. 10 for the angle of attack of $\alpha=0^{\circ}$.

For the two-dimensional Menter-SST simulation (orange line) the pressure distribution shows a plateau in the rear part of the flap as in the experimental data. Since the onset of the separation is to early as was shown in Fig. 6 the plateau

In Joint Symposium "Simulation of Wing and Nacelle Stall", Jun. 21-22, 2012, Braunschweig, Germany, 2012 


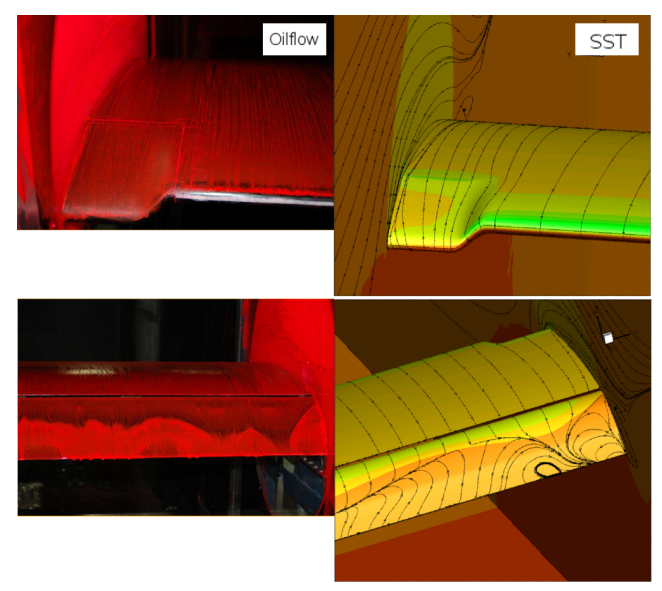

Fig. 9: Oil-flow pictures and skin-friction lines from Menter-SST model at $\alpha=0^{\circ}$

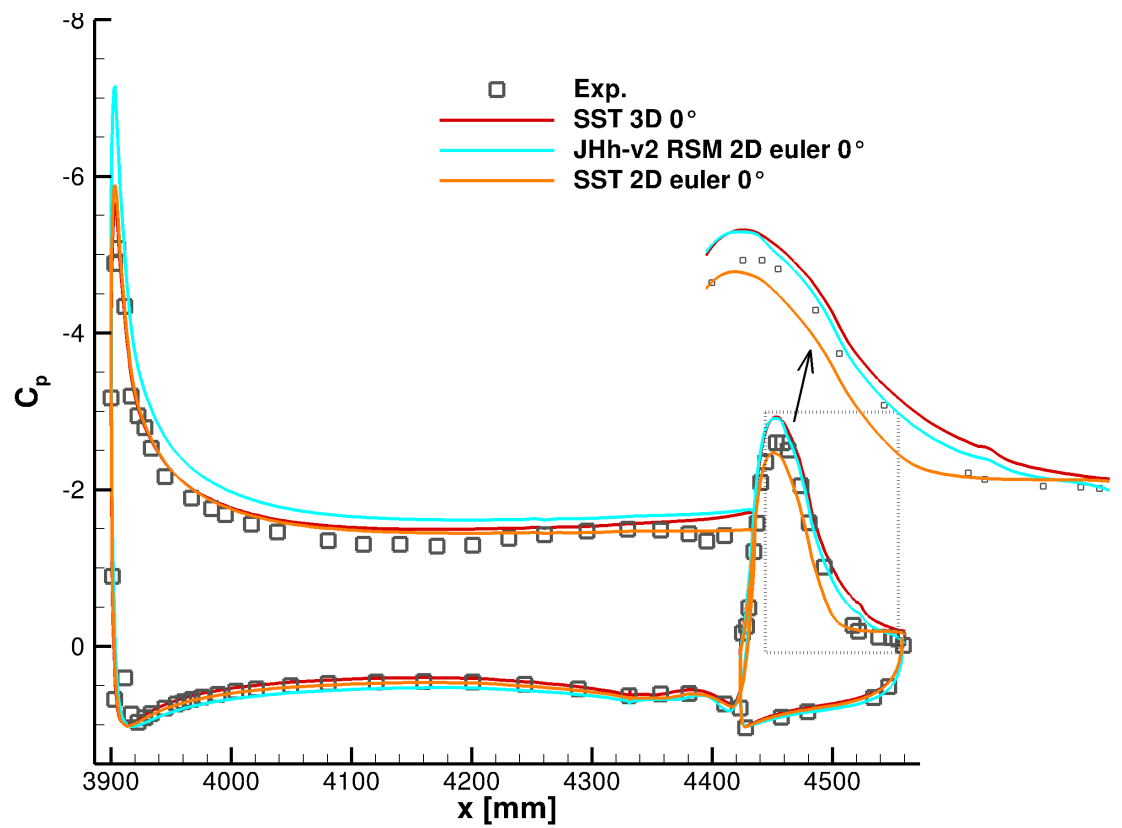

Fig. 10: Comparison of the mean-pressure distribution obtained with different turbulence models and numerical setups at $\alpha=0^{\circ}$

builds too early and the suction peak is predicted too low. No such plateau can be seen in the two-dimensional JHh-v2 RSM simulation (light blue line) due to the attached flow on the flap. Therefore, the circulation is predicted wrong in the RSM simulation and the suction peaks are over-predicted on both elements and a larger offset from the experimental data can be observed at the main wing. The threedimensional SST simulation (red line) predicts separation at the centerline section but the separation point is shifted downstream compared to the experimental data. In the mean-pressure distribution only a very small plateau can be seen close to the trailing edge of the flap. Therefore, the pressure distribution is much closer to the

In Joint Symposium "Simulation of Wing and Nacelle Stall", Jun. 21-22, 2012, Braunschweig, Germany, 2012 
RSM on the flap. On the main wing however, the difference between the two- and three-dimensional SST simulations is very small. To further assess these results an additional three-dimensional simulation using the JHh-v2 RSM model is planned.

For the $\alpha=6^{\circ}$ case more simulations are compared in Fig. 11 and the outcome is more clear. Here, the third approach, the two-dimensional simulation with boundary layer resolution at the upper and lower wind tunnel wall, is included. For this approach the result from the Menter-SST model is presented.

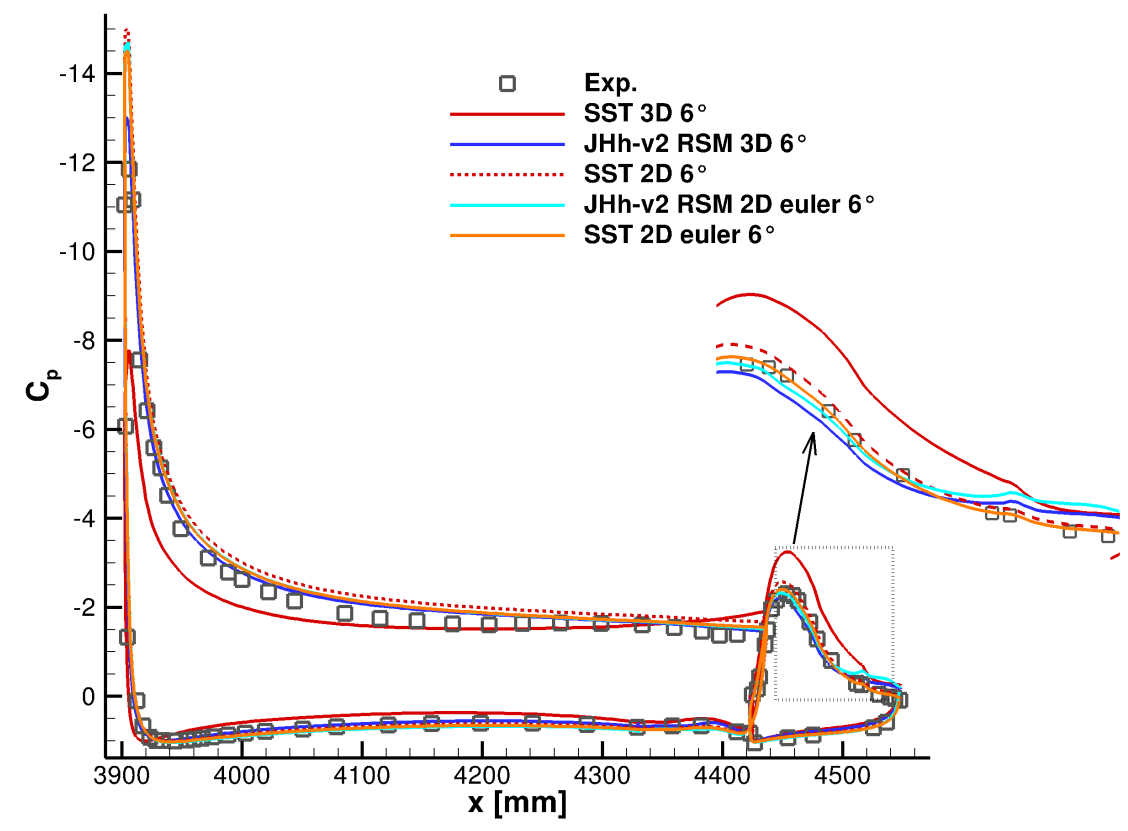

Fig. 11: Comparison of the mean-pressure distribution obtained with different turbulence models and numerical setups at $\alpha=6^{\circ}$

The pressure distribution of the two-dimensional Menter-SST simulation with inviscid tunnel walls (orange line) is in very good agreement with the experimental data. The results that were obtained in the two-dimensional approach with boundary-layer resolution with the SST model (dashed red line) show a little offset from the former results but the general behavior is not changed. Only the pressure distribution in the centerline section of the three-dimensional simulation using the Menter-SST model is completely different. This is due to the massive separation that is predicted at the side walls with this model.

The results from the two-dimensional JHh-v2 RSM simulation with inviscid tunnel walls (light blue line) almost coincide with the corresponding SST results on large parts of the profile. Only the instability above the flap leads to a bigger deviation from the experimental distribution for the RSM model. The pressure distribution in the centerline section of the three-dimensional simulation with the RSM model (blue line) is very close to the two-dimensional results.

In Joint Symposium "Simulation of Wing and Nacelle Stall", Jun. 21-22, 2012, Braunschweig, Germany, 2012 


\section{Conclusion}

Three different approaches to model the influence of the wind tunnel walls were compared. For the least expensive approach, neglecting the boundary layers at the tunnel walls and restricting the simulation to the centerline section of the wind tunnel, the grid resolution and time-step size were evaluated. Here, it was found that using the JHh-v2 RSM model leads to instabilities in the wing cove. A fast decay of the Reynolds-stresses behind the separation point was found to be responsible for this behavior.

In the three-dimensional simulation at an angle of attack of $\alpha=6^{\circ}$ the Menter-SST model predicted a massive separation of the flow at the junction between the profile and the wind tunnel wall that disturbed the flow even at the centerline section. However, for an angle of attack of $\alpha=0^{\circ}$ the results with the Menter-SST model looked much better. The JHh-v2 RSM model was only applied for $\alpha=6^{\circ}$ and the flow at the model-tunnel junction was predicted in accordance with the experimental data.

Comparing the pressure distribution at an angle of attack of $\alpha=6^{\circ}$, the following could be observed: The influence of the boundary layer on the upper and lower wind tunnel wall in a two-dimensional simulation is very small. This could be shown for the Menter-SST model. The results using the JHh-v2 RSM model show good agreement between the centerline section of the three-dimensional simulation and the two-dimensional simulation with inviscid walls.

These results show that it is appropriate to neglect the influence of the wind tunnel walls in a simulation as long as the experiments are designed to minimize the side wall effects. The droop noses that were used in the present experiments are a good approach to achieve this goal in the case of multi-element airfoils.

In the future it is planned to apply turbulence resolving models to this test case. With respect to these simulation the current results are very important. Resolving the entire three-dimensional setup in a scale resolving approach is not feasible with the currently available computational resources. Based on this work it is now justified to restrict the computational domain to the centerline section of the wind tunnel.

\section{Acknowledgments}

The members of the FOR 1066 research group gratefully acknowledge the support of the "Deutsche Forschungsgemeinschaft DFG" (German Research Foundation) which funded this research.

\section{References}

[1] Cecora, R.-D., Eisfeld, B., Probst, A., Crippa, S., and Radespiel, R., "Differential Reynolds Stress Modeling for Aeronautics," AIAA Paper 2012-0465, January 2012.

[2] Hahn, D., Scholz, P. and Radespiel, R.: "Experimental evaluation of the stall

In Joint Symposium "Simulation of Wing and Nacelle Stall", Jun. 21-22, 2012, Braunschweig, Germany, 2012 
characteristics of a two-element high-lift airfoil". Second Symposium "Simulation of Wing and Nacelle Stall" June 22nd - 23rd, Braunschweig, Germany, 2010 .

[3] Schwamborn, D., Gerhold, T. and Heinrich, R.: "The DLR TAU-Code: recent applications in reaserach and industry". In "proceedings of ECCOMAS CFD 2006, Wesseling, P. Oñate, E. Périaux, J. (eds.), The Netherlands, TU Delft", 2006.

[4] Menter, F. R.: "Two-Equation Eddy-Viscosity Turbulence Models for Engineering Applications". AIAA Journal, Vol. 32, No. 8, pp. 1598-1605, August 1994.

[5] Jakirlić, S. and Hanjalić, K.: "A new approach to modelling near-wall turbulence energy and stress dissipation". Journal of Fluid Mechanics, 459:139-166, June 2002 .

In Joint Symposium "Simulation of Wing and Nacelle Stall", Jun. 21-22, 2012, Braunschweig, Germany, 2012 


\title{
$[\mathrm{F}]$
}

\section{Hybrid RANS/LES Study of the Development of an Airfoil-Generated Vortex}

\author{
S. Reuß ${ }^{1}$, A. Probst ${ }^{1}$, T. Knopp ${ }^{1}$, and K. Wawrzinek ${ }^{2}$ \\ ${ }^{1}$ Institute of Aerodynamics and Flow Technology, DLR, Bunsenstr. 10, 37073 Göttingen, \\ Germany \\ ${ }^{2}$ Institute of Aerodynamics and Gas Dynamics, Universität Stuttgart, Pfaffenwaldring 21, \\ 70569 Stuttgart, Germany
}

\begin{abstract}
Published in:
Radespiel, R. et al (Eds.): Advances in Simulation of Wing and

Nacelle Stall, Notes on Numerical Fluid Mechanics and Multidisciplinary Design, Vol. 131, pp. 41-54, Springer, 2015
\end{abstract}

\begin{abstract}
We consider the numerical simulation of a generic lateral vortex, that is generated by a rapidly deflected NACA0021 airfoil. We use the SST $k-\omega$ URANS approach and a hybrid RANS-LES method based on the SST model, where a zonal RANSLES interface is located little downstream of the airfoil trailing edge. The results are assessed by reference with the experimental data. The mean flow behavior and the induced angle of attack of the vortex can be predicted satisfactorily by the URANS approach and the improvement using hybrid RANS/LES is small. The hybrid RANS/LES results exhibit a delayed shear layer instability of the wake, and the arising $2 \mathrm{D}$ roller type vortices lead to a significant overprediction of the turbulent shear stress. We then apply a stochastic forcing in the region of the airfoil trailing edge. This leads to a fast generation of turbulent content in the wake and clearly improves the predictions for the turbulent stresses.
\end{abstract}

\section{Introduction}

The reliable prediction of an aircraft in high-lift configuration near stall using computational fluid dynamics is still an open problem, especially in case of non-homoge- 
neous, i.e., disturbed, inflow conditions. In the joint research project FOR-1066 we are interested in disturbances, whose characteristic length scales cover a range from the airfoil chord length down to the small-scale turbulence. The goal is to identify which length scales have the dominant effect on the stall behavior [9].

In order to study this question numerically, we need (i) a simulation method for predicting airfoil stall, (ii) a method to account for large-scale disturbances in the onflow and (iii) a test case with experimental reference data to validate the simulation method.

Concerning (i) we use hybrid RANS/LES methods (HRLM) to simulate the flow over multi-element airfoils near stall for homogeneous onflow. A modification of the Delayed Detached Eddy Simulation (DDES) method [14] has been conceived and was further developed during the project: the Algebraic DDES (ADDES) [5], [8] and its improvements are described in section 2.1. With this method we want to assess the role of the small-scale structures on the boundary-layer physics and its separation behavior in the case of a disturbed onflow and compare the results with Reynolds-stress model (RSM) predictions.

For (iii) we need a test case at a level of complexity and computational cost which are feasible. Within the FOR-1066, a carefully designed, unique validation experiment was performed [4]. A NACA0021 vortex-generator airfoil (VG) is mounted at $\alpha=0^{\circ}$ into the first part of the test section. At a time $t_{0}$ the airfoil is rapidly deflected by $\Delta \alpha=10^{\circ}$ in a well defined motion. As the VG airfoil is pitching up, the change in circulation causes the generation of a layer of vorticity in its wake which partly rolls up to a transversal vortex. This generic vortex is convected with the free-stream velocity. In the preparation phase of the experimental campaign, the generation and the evolution of the generic vortex in the empty wind-tunnel test section was studied. In the main experiment, a DLR F15 two-element high-lift airfoil (HL) is mounted downstream of the trailing edge of the VG airfoil and the interaction of the vortex with the HL airfoil is studied.

The present work is dedicated to (ii). We consider the generation and the evolution of the generic vortex in the empty test section. We apply the scale-resolving HRLM approach and compare the results with unsteady RANS (URANS) results and with the experimental data. The generation and evolution of the vortex without and with interaction with the HL airfoil was studied using URANS simulations in [12]. Within the FOR-1066 the influence of the VG position has been investigated in [16].

\section{Hybrid RANS/LES Method}

We use the DLR TAU code, an unstructured finite volume solver for the compressible Navier Stokes equations. We use the low-dissipation setting from [6] to improve the accuracy of the method for scale-resolving simulations. For hybrid RANS-LES simulations, several background RANS turbulence models are supported, e.g., the SA-model, the SST $k-\omega$ model, and the JHh RSM. Moreover, various RANS/LES models like DES, DDES and IDDES as well as ADDES are provided.

Radespiel et al (Eds.): Advances in Simulation of Wing and Nacelle Stall, NNFM 131, pp. 41-54 Springer, 2015 


\subsection{Improvements of the Algebraic Delayed DES Method}

In some flow situations involving a strong adverse pressure gradient, DDES does not ensure the RANS mode in the entire boundary layer, which can lead to premature separation [7]. On the other hand, DDES can fail to detect thin separation regions and undesiredly treat them in the RANS mode instead of the LES mode. Therefore, the basic ADDES [5], [8] was developed for single element airfoils. In the ADDES several algebraic sensors are available to identify RANS and LES regions: The velocity profiles in the boundary layer are analyzed along wall-normal lines in order to determine the outer edge of the boundary layer and to detect flow separation. If the shape-factor $H_{12}$ exceeds a model dependent critical threshold $H_{12 \text {,crit }}$, the flow in the boundary layer is assumed to be separated. These sensors are used to control the switching method between RANS and LES regions.

Several extensions were needed for the application scenario of the flow over a twoelement airfoil at homogeneous and at disturbed inflow conditions. Firstly, the algebraic sensors to detect regions of attached boundary layers, separation and reattachment were reviewed for additional test cases, e.g., the separated flow behind a wall-mounted hump, which support the previous results. Moreover, the implementation of the sensors was extended to allow for massive parallel simulations.

Additionally, we addressed the so-called grey-area problem typical for HRLM: after the transition from RANS to LES the generation of resolved turbulence is delayed. To mitigate this delay, the proposal of a modified length scale $\Delta_{\omega}$ in the LES region from [1] was considered. Therein, the orientation of the local vorticity vector w.r.t. the control volume is taken into account for determining the LES length scale. This approach was generalized to a formulation, which is suited for general cell types in an unstructured code: $\Delta_{\omega}=\sqrt{\sum_{i} \boldsymbol{N} \cdot \boldsymbol{s}_{i}}$, where $\boldsymbol{N}$ is the normalized vorticity vector and $s_{i}$ is the area-weighted normal vector of a dual cell face $i$.

Another way to resolve the grey-area problem is to add synthetically generated turbulence at the RANS/LES interface. Therefore, we provided a flexible infrastructure to introduce the synthetic turbulence in several arbitrary forcing volumes in the ADDES. The algebraic sensors can be used to define the forcing volumes automatically. This infrastructure was used by project partners in FOR-1066 to implement the Synthetic Turbulence Generator (STG), and the effectiveness of the method was shown in $[2],[3]$.

Moreover, we observed for the DDES of the flow around the DLR F15 three-element airfoil [10], that unsteady vortical flow from the wing cove can penetrate into the boundary layer on the flap. This can lead to a significant delay of flow separation on the flap. Two solution strategies were developed during this work, based on a sensor that recognizes turbulent boundary layers. In the case of sufficient mesh resolution we make use of the wall-modelled LES capability of the Improved DDES (IDDES) [15]. For this we coupled the algebraic sensors with the IDDES model. However, the mesh resolution requirement for a wall-modelled LES at high Reynolds numbers can be prohibitively high. Therefore, an alternative approach suited for simulations with a lower grid resolution is pursued as well. In order to avoid an under-resolved LES and to ensure a pure RANS solution, the ADDES is currenly beeing extended by a model for the transition from LES to RANS. The approach uses sponge layers to transfer resolved turbulence from the LES region into modelled Reynolds stresses in the RANS region.

Radespiel et al (Eds.): Advances in Simulation of Wing and Nacelle Stall, NNFM 131, pp. 41-54 Springer, 2015 


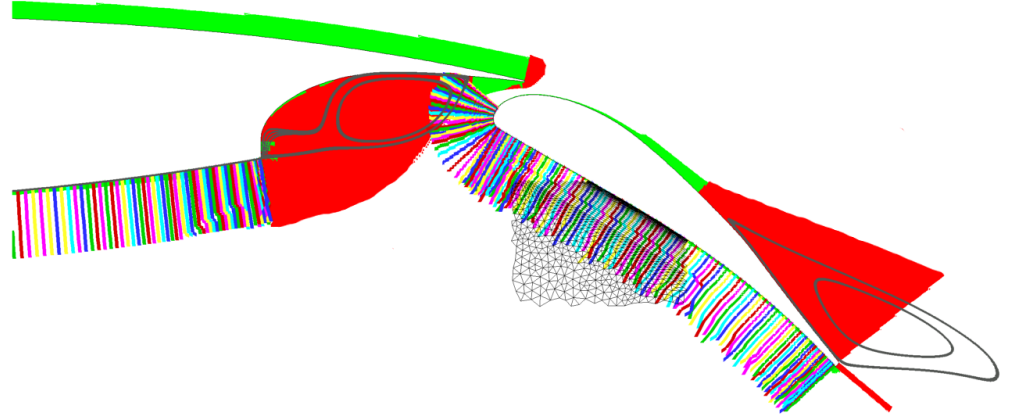

Fig. 1: Application of the ADDES to the DLR F15 two-element airfoil: the wall-normal lines are shown on the lower side, RANS regions are colored in green and separated regions are colored in red.

An additional aim is to ensure a mesh resolution in the LES regions that resolves a sufficient part of the turbulence spectrum. Therefore, we apply a local LES resolution sensor which measures the ratio of the resolved turbulent kinetic energy (TKE) $k_{\text {res }}$ to the total TKE $k_{\mathrm{tot}}=k_{\mathrm{res}}+k_{\mathrm{mod}}$. In [13] several formulations for the modelled TKE are compared and the TKE sensor is used as an input variable for a local mesh refinement using the adaptation tool that is available in the DLR TAU code: in LES regions the grid is refined, if less then $80 \%$ of the total TKE are resolved, because this is regarded as underresolved LES.

\subsection{Applicability of the ADDES to a High-Lift Airfoil}

The applicability of the method to the flow over the DLR F15 two-element airfoil is demonstrated in Fig. 1. On the pressure side the wall-normal lines used to evaluate the algebraic boundary-layer sensors are shown. As indicated on the lower side of the flap, the lines are not confined to the near-wall structured layers of the grid, but can be used also in regions of tetrahedral elements. The green area shows the parts of the boundary layers where the algebraic sensors of the ADDES ensure RANS mode. The regions of separated flow are marked in red, e.g. the separation on the flap and in the cove of the main wing element. In those regions the ADDES switches into the LES mode. Note, that behind the trailing edges of the main wing and the flap the sensors detect separation, and the model is switched to LES to counter the grey-area problem.

In order to reduce the computational costs, we designed a strategy to generate efficient 2D-3D meshes using the adaptation module of TAU. To illustrate this, consider the situation in which the LES mode is active only in the wing cove and on the flap. Then a coarse mesh spacing in spanwise direction upstream of the wing cove is sufficient, since only RANS mode is used there. In the region of the wing cove and on the flap the mesh is anisotropically refined in the spanwise direction to match the required LES resolution. Bridging elements are introduced between the elements of different spanwise resolution. In Fig. 2 the situation is depicted. On the left, the transition elements on the surface are shown. Within seven refinement steps the number of cells in the spanwise direction is increased from one to 128 . Here, the

Radespiel et al (Eds.): Advances in Simulation of Wing and Nacelle Stall, NNFM 131, pp. 41-54 Springer, 2015 


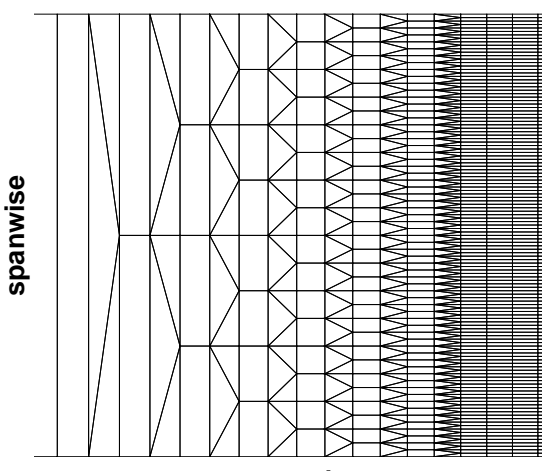

streamwise

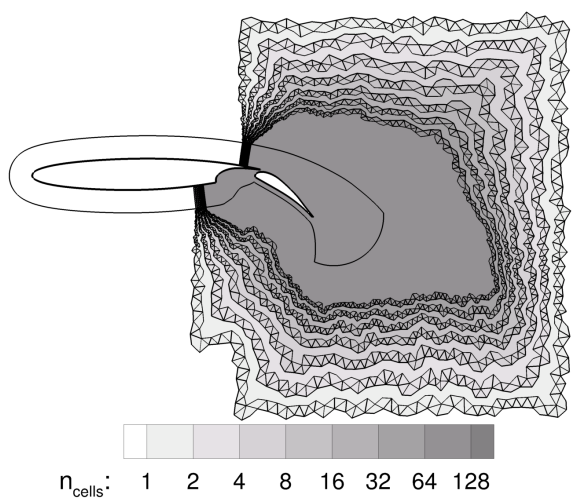

Fig. 2: Efficient 2D-3D grid around the DLR F15 two-element airfoil, obtained through grid refinement in the spanwise direction.

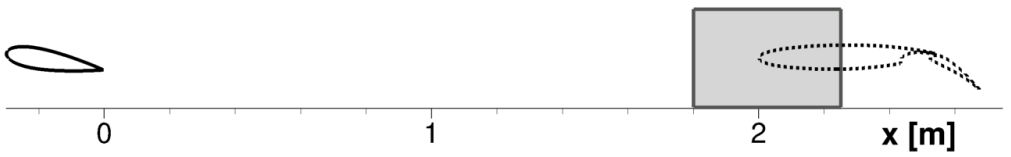

Fig. 3: Experimental setup: the position of the HL airfoil and the PIV window are marked.

quadrilateral elements on the surface are split into bridging triangles. On the right in Fig. 2 the transition elements in the refinement region are outlined in a spanwise cut. The number of cells in the spanwise direction is indicated by the grey shades. The single black line shows the outer bound of the near-wall structured grid layers. In the efficient 2D-3D grid $35 \%$ of points are saved compared to a grid that is globally refined in the spanwise direction.

\section{Simulation of the vortex generation and transport in a wind tunnel}

The experimental setup is depicted in Fig. 3. The wind tunnel is operated at $u_{\text {ref }}=50 \mathrm{~m} / \mathrm{s}$ yielding a Reynolds number of $R e=1.9 \cdot 10^{6}$ with respect to the retracted chord length of the high-lift airfoil $l_{\text {ref }}=0.6 \mathrm{~m}$. The vortex generator airfoil has a chord length of $c_{\mathrm{VG}}=0.3 \mathrm{~m}$. In the target setup the distance from the trailing edge of the VG airfoil to the leading edge of the HL airfoil is $2 \mathrm{~m}$. The position of the HL airfoil in the main experiment is marked. In the same position the PIV window is outlined, where the data were measured in the preparation phase without the HL airfoil.

In the simulations, the spanwise centerline section of the wind tunnel is considered. It was shown in [11] that the influence of the wind-tunnel side walls on the results can be neglected. The computational domain has a length of $5.6 \mathrm{~m}$ and a height of

Radespiel et al (Eds.): Advances in Simulation of Wing and Nacelle Stall, NNFM 131, pp. 41-54 Springer, 2015 


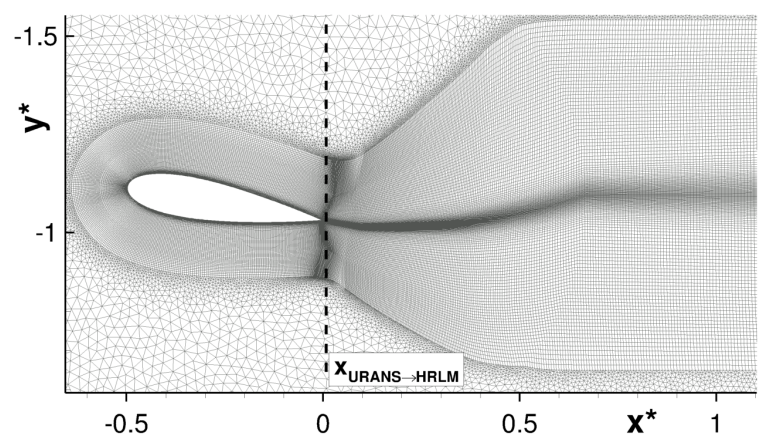

Fig. 4: Vortex generator grid with structured transition to vortex transport region. The grid has been deformed such that the VG is at an incidence of $\alpha=10^{\circ}$

$1.3 \mathrm{~m}$. The upper and lower wind-tunnel walls are modelled as inviscid slip-walls, at the inflow and outflow farfield boundary conditions are used. From the experimental data a vortex diameter of $d / c_{\mathrm{VG}}=0.83$ is estimated. In order to cover it entirely, $L_{z} / c_{\mathrm{VG}}=1$ is chosen for the spanwise extent of the computational domain. The $x, y$ and $z$-coordinates correspond to the streamwise, wall-normal and spanwise directions respectively, and the origin of the computational domain is located at the trailing edge of the VG airfoil at $\alpha=0^{\circ}$.

The grid is shown in Fig. 4: 400 points are located on the surface of the VG airfoil and 80 wall-normal layers of hexahedral elements cover the structured region that transitions smoothly into the vortex transport region. Here, the cells have a size $\Delta_{x} / c_{\mathrm{VG}}=\Delta_{y} / c_{\mathrm{VG}}=0.017$ except for the immediate wake region where the cells have an aspect ratio of $\Delta_{x} / \Delta_{y}=4$. With 60 cells in the spanwise direction and a cell size of $\Delta_{z} / c_{\mathrm{VG}}=0.017$, the transport region is resolved with isotropic cells.

All simulations are based on the SST $k-\omega$ model. The region of the VG airfoil is always simulated in URANS mode. In the HRLM simulations the DES mode is manually activated at $x_{\mathrm{URANS} \rightarrow \mathrm{HRLM}} / c_{\mathrm{VG}}=0.017$ behind the trailing edge of the VG airfoil. In order to achieve the deflection of the VG airfoil, the deformation tool in TAU is used after each solver step to deform the mesh. In the following all values are normalized with the reference values, i.e. $u^{*}=u / u_{\text {ref }}, y^{*}=y / l_{\text {ref }}$ and $t^{*}=t / t_{\text {ref }}$, with $t_{\text {ref }}=l_{\text {ref }} / u_{\text {ref }}$.

\subsection{Evaluation of statistics for time-dependent flow problems}

In the experiments the streamwise and wall-normal velocity components were measured using $2 \mathrm{~d}$ particle image velocimetry (PIV). In several positions time-resolving data were recorded for the velocity magnitude and the induced angle of attack with a five-hole probe (FHP). In order to obtain phase-locked averaged data the deflection of the NACA profile was repeated periodically with a down- and an upstroke. The FHP data is averaged over 90 periods, and the PIV data are averaged over 300 repetitions of the experiment.

In general, it is not feasible to repeat a scale-resolving simulation this often to compute the ensemble/phase averages. Instead the mean values and fluctuations need

Radespiel et al (Eds.): Advances in Simulation of Wing and Nacelle Stall, NNFM 131, pp. 41-54 Springer, 2015 


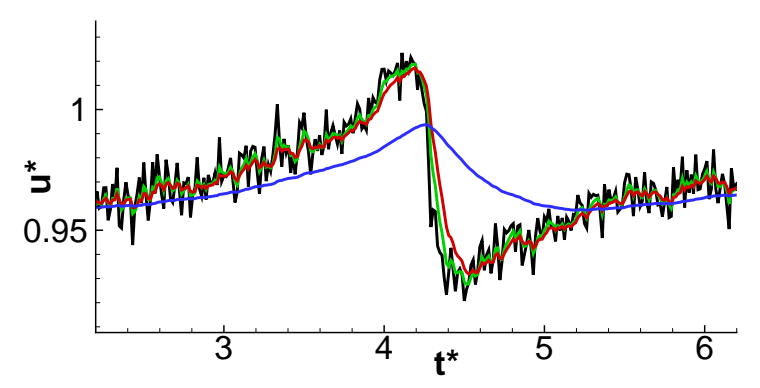

Fig. 5: Three weights for the WMA mean values are compared for the experimental FHP velocity: original data $\longrightarrow ; w(25) \longrightarrow ; w(50) \longrightarrow ; w(500)-$

to be obtained from a single simulation. In the presence of instationary inflow conditions, the mean values in the simulation are computed with a weighted movingaverage (WMA) formulation:

$$
\langle u\rangle_{t}=\langle u\rangle_{t-1}+w(s) *\left(u(t)-\langle u\rangle_{t-1}\right),
$$

where the influence of the old samples decays exponentially through the weighting factor $w(s)=2 /(s+1)$.

In order to assess $w(s)$, the WMA mean values of the experimental FHP data using three different weights are compared in Fig. 5. Too small weights $(w(25))$ lead to strong oscillations, whereas with too large values $(w(500))$ the minima and maxima are smeared. Here, the smoothest WMA mean values are obtained using $w(50)$.

In order to obtain relevant values for the variances (e.g. Reynolds stresses) the mean values should be statistically converged. Therefore, the number of samples is virtually increased by assuming that the flow at different spanwise locations can be regarded as different realizations of the same experiment. Thus, instead of taking the ensemble average the spanwise average is used to improve the mean values.

\subsection{Assessment of the HRLM}

In the basic simulation (HRLM-basic) the shear layer behind the VG airfoil at $\alpha=0^{\circ}$ is very stable and two-dimensional rollers develop. The deflection of the vortex generator alone is not sufficient to induce enough instability to break up the rollers into 3D turbulence, cf. Fig. 6 (top). To provoke the breakup of the 2D rollers, following approaches were tested: The grid was refined in the spanwise direction by a factor of 4 , the whole wake region was further refined, and the time step was reduced by a factor of 10, all without success. Finally, a successful simulation was achieved by exciting the wake artificially using a stochastic forcing approach (HRLM-forced): In the region of the VG trailing edge the eddy viscosity is multiplied by a random factor $c_{\text {rand }} \in[0,3]$ with unity expectation value $E\left(c_{\text {rand }}\right)=1$. The region starts at $85 \% c_{\mathrm{VG}}$, where the URANS mode is active, and has a wall-normal extent of $15 \%$ $c_{\mathrm{VG}}$ around the trailing edge, so that it is also effective in a small region downstream of $x_{\text {URANS } \rightarrow \text { HRLM }}$ where the simulation is switched to DES.

In Fig. 6 the turbulent structures in the wake are visualized by an isosurface of the second invariant of the velocity-gradient tensor $Q_{i n v}$. The snapshot is taken at

Radespiel et al (Eds.): Advances in Simulation of Wing and Nacelle Stall, NNFM 131, pp. 41-54 Springer, 2015 


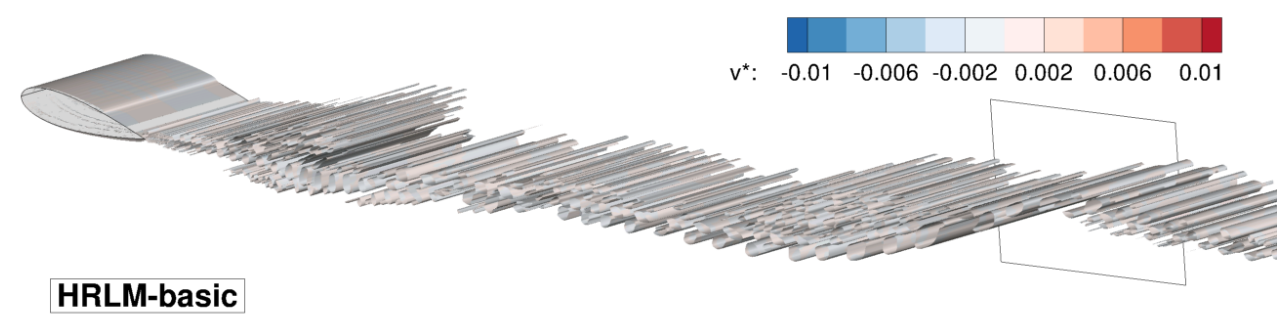

HRLM-basic

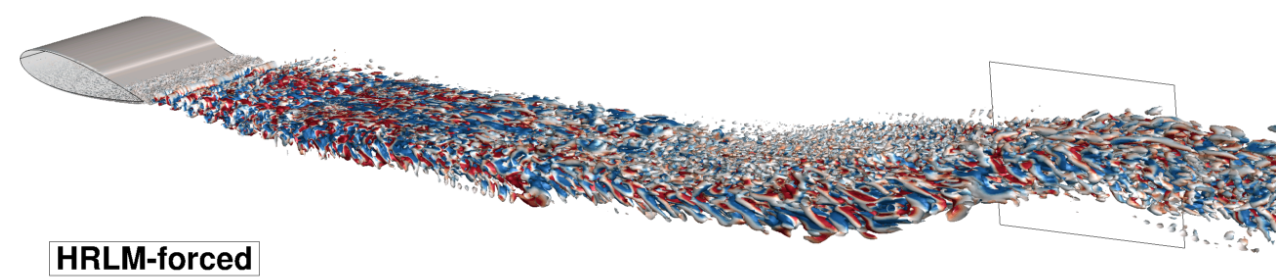

Fig. 6: Isosurface of $Q_{i n v}$ to illustrate the turbulent structures at $t^{*}=4.16$ in the basic HRLM (top) and in the HRLM with stochastic forcing (bottom).

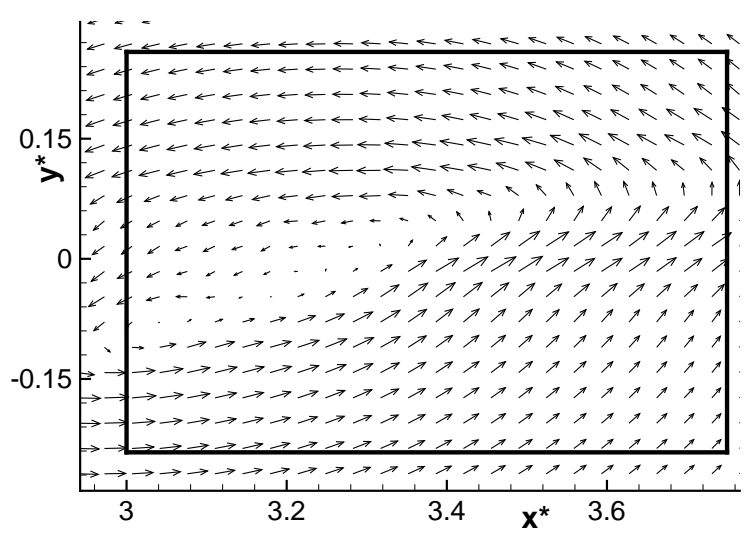

Fig. 7: Visualization of the position of the vortex at $t^{*}=4.16$ through the velocity difference vectors.

$t^{*}=4.16$ when the vortex has reached the PIV window that is outlined in the side plane. The vortex in the HRLM-basic computation (top) consists of $2 \mathrm{D}$ rollers. In the HRLM-forced computation (bottom) the 2D rollers break up close downstream of the trailing edge, and 3D turbulent content develops quickly.

To identify the vortex, the velocity difference vectors $u^{\prime}=\langle u\rangle_{t^{*}=4.16}-\langle u\rangle_{t^{*}=0}$ are plotted in Fig. 7. The position of the PIV window is indicated by the thick line. The core of the vortex consists of two distinct centers of rotation at $\left(x^{*}, y^{*}\right)=$ $(3.05,-0.08)$ and at $\left(x^{*}, y^{*}\right)=(3.2,-0.03)$.

Radespiel et al (Eds.): Advances in Simulation of Wing and Nacelle Stall, NNFM 131, pp. 41-54 Springer, 2015 

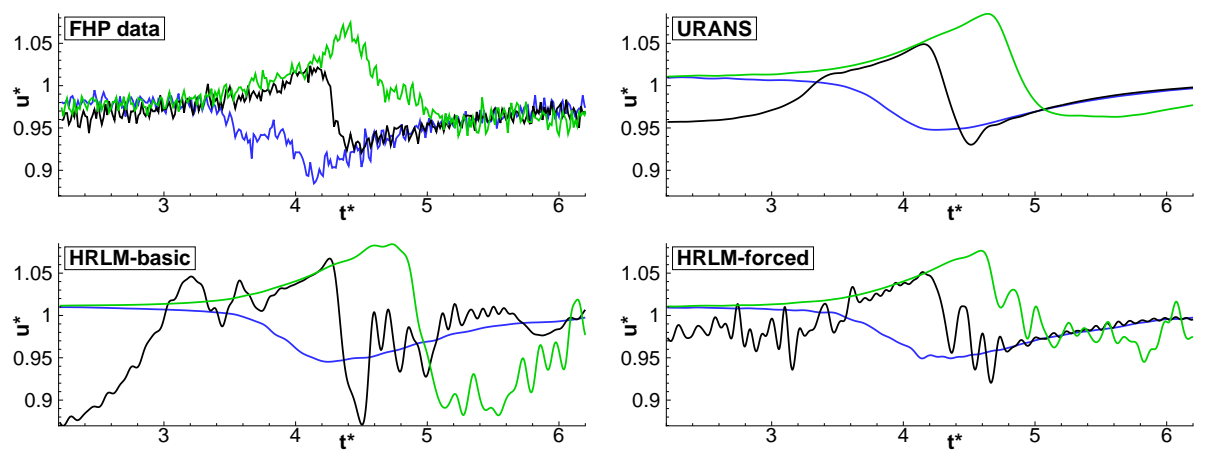

Fig. 8: Influence of the vortex on the velocity magnitude at three positions: $y^{*}=0.17-$; $y^{*}=0.0-; y^{*}=-0.17-$.

\subsection{Comparison with experimental data}

First we consider the velocity $u^{*}$ and the induced angle of attack $\alpha$. In the simulations they are evaluated at three wall-normal probe positions at $x^{*}=3.5$ : in the center of the wake of the VG airfoil in the initial position at $y^{*}=0.0$, above at $y^{*}=0.17$ and below at $y^{*}=-0.17$. In the experiment the FHP data were measured at the same positions. The results are shown in Figs. 8 and 9 . The experimental data are phase-locked averages over 90 cycles whereas no averaging was performed on the simulation data. For $u^{*}$ and $\alpha$ periodic fluctuations with a frequency $f=950 \mathrm{~Hz}$ can be observed in both hybrid simulations. With the transport velocity $u_{\text {ref }}$ the corresponding structure size $l=0.05 \mathrm{~m}$ is 10 times larger than the local cell size and corresponds to the average distance between the 2D rollers in the HRLM-basic case. In the HRLM-forced case the correlation coefficient in the spanwise direction does not drop below 0.6 (not shown here), which indicates that the $2 \mathrm{D}$ roller character is partly maintained in this simulation.

In Fig. 8 an offset between the velocity magnitude in the simulations and the experimental data can be observed at all three probe-positions. This indicates that the mean velocity in the wake is higher in the simulations. However, in the center position at $y^{*}=0$ the maximum value is reached at the same time $t^{*}=4.17$ in all curves, which proves that the vortex is transported with the same convection velocity in the experiment and all simulations. The maximum value in $y^{*}=-0.17$ is reached at $t^{*}=4.4$ in the experiment, whereas in all simulations it is predicted at a later time, indicating that the vortex roll-up is not as strong as in the experiment. In the HRLM-basic case the large 2D rollers lead to strong distortions in the center position $y^{*}=0.0$.

The measurements of the induced angle of attack $\alpha$ in Fig. 9 exhibit a steep gradient during the vortex encounter which is not reproduced by the simulations. In the center position at $y^{*}=0$ the experiments show a minimum which is not predicted by any of the simulations, whereas the location and strength of the maximum agrees with the experimental data for all simulations. Also in the other positions the magnitude of the induced incidence angle is predicted in good agreement with the experiments by all approaches.

In summary, the velocity magnitude $u^{*}$ and the induced angle of attack $\alpha$ show a

Radespiel et al (Eds.): Advances in Simulation of Wing and Nacelle Stall, NNFM 131, pp. 41-54 Springer, 2015 

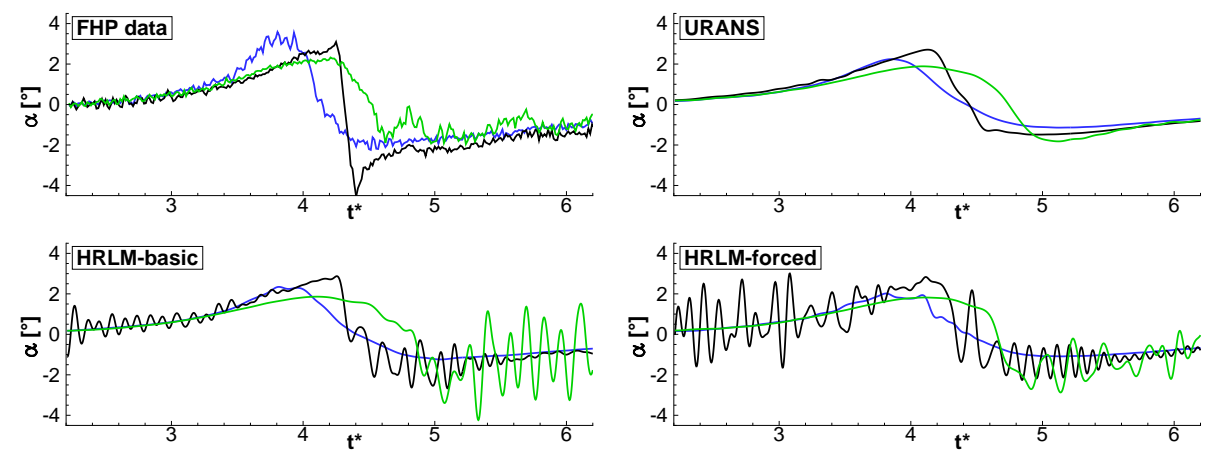

Fig. 9: Influence of the vortex on the induced incidence angle at three positions: $y^{*}=0.17-; y^{*}=0.0-; y^{*}=-0.17-$.
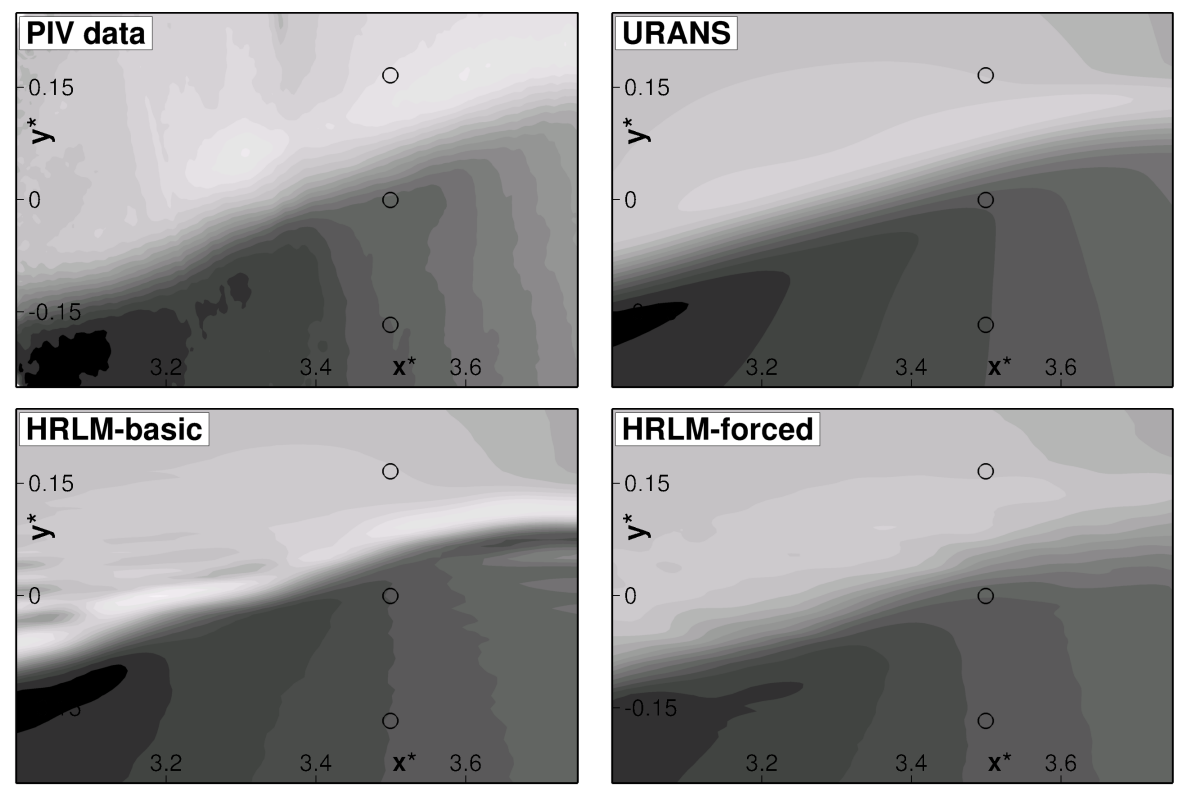

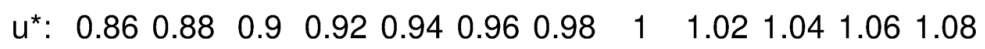

Fig. 10: Mean streamwise velocity around the FHP positions (black circles) at $t^{*}=4.16$

close agreement in the mean vortex-transport characteristics for URANS and HRLM. In Fig. 10 the mean streamwise velocity fields $\left\langle u^{*}\right\rangle$ at $t^{*}=4.16$ from the simulations are compared with PIV measurements. The FHP positions are marked by the circles, for reference. In the PIV the streamwise gradient is steeper and the inclination of the wake is stronger. This again shows that the strong vortex roll-up in the experiments is not predicted in the simulations. Moreover, in the experiment two distinct minimum regions can be recognized well above the shear layer. In the URANS and the HRLM-forced computations these regions are not captured. This might partly be a

Radespiel et al (Eds.): Advances in Simulation of Wing and Nacelle Stall, NNFM 131, pp. 41-54 Springer, 2015 

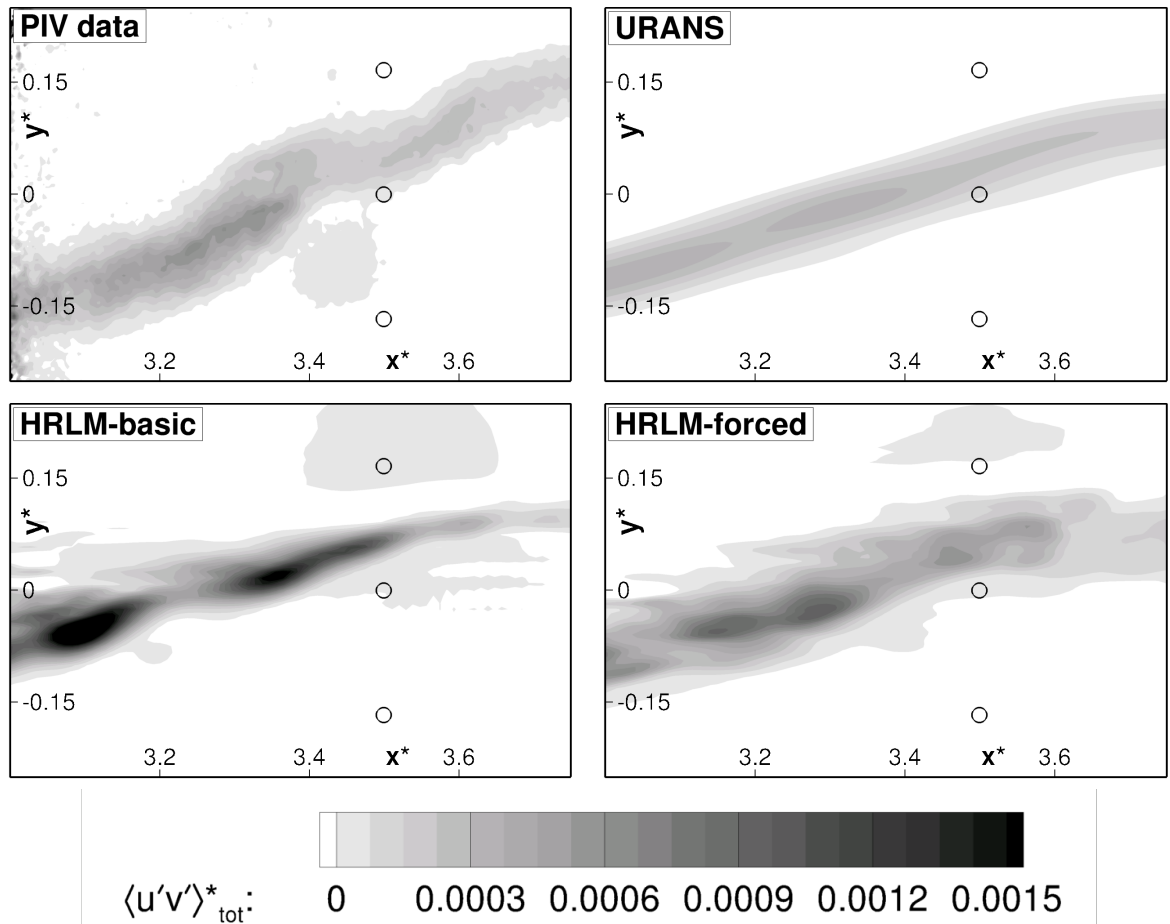

Fig. 11: Mean total turbulent shear stress around the FHP positions (black circles) at $t^{*}=$ 4.16

smearing effect of the WMA averaging and could be further amplified by the spanwise averaging. In the HRLM-basic case the impact of the large 2D rollers produces minimal values similar to the measured ones.

Alltogether, the WMA mean values show a good qualitative agreement with the experiment. With respect to the averaging procedure in sec. 3.1 they seem to be suited to compute reasonable velocity fluctuations for the evaluation of the resolved turbulence.

In Fig. 11 the total turbulent shear stress is depicted: $\left\langle u^{\prime} v^{\prime}\right\rangle_{\text {tot }}=\nu_{t}\left(\frac{\partial u}{\partial y}+\frac{\partial v}{\partial x}\right)-$ $\left\langle u^{\prime} v^{\prime}\right\rangle_{\text {res. }}$. In the URANS simulation only the modelled part is shown, even though in the simulation the resolved part is of the same order. This can be explained by the averaging approach: the influence of the old values causes a deviation between the WMA mean and the true mean that would be obtained by ensemble averaging. It can be assumed that with the true mean value, RANS would predict no resolved turbulence, so $\left\langle u^{\prime} v^{\prime}\right\rangle_{\text {res }}^{\mathrm{RANS}}$ represents a systematic error in the statistical post-processing procedure. The same systematic deviation exists for the HRLM approaches, however, it is not clear whether it is possible to transfer the quantification of the error directly.

In both hybrid approaches the level of modelled shear stress is negligible. The level of total turbulent shear-stress in the HRLM-forced computation is in acceptable agreement with the experimental data. In the HRLM-basic case the large 2D rollers lead to an overestimation of the turbulent shear stress by a factor of 4 . Thus, only

Radespiel et al (Eds.): Advances in Simulation of Wing and Nacelle Stall, NNFM 131, pp. 41-54 Springer, 2015 
the HRLM-forced approach is considered suitable to predict the relevant turbulent scales in the transported vortex.

\section{Conclusion and Outlook}

A method to simulate the generation and transport of a generic vortex using a hybrid RANS/LES simulation has been presented. It has been shown that it is necessary to introduce artificial disturbances to break up the wake of the vortex generator airfoil into 3D turbulence. A combination of a weighted moving-average formulation and spanwise smoothing has successfully been used to provide mean-flow statistics for the unsteady process.

It has been shown that the mean vortex characteristics can be captured with a RANS simulation and that the hybrid RANS/LES method yields the same mean characteristics. It has been shown that HRLM with forcing predicts the resolved turbulence in good agreement with the experiments, while without the forcing, large $2 \mathrm{D}$ rollers lead to an overestimation of the turbulent shear stress.

This work was the final step on the way to our target application: In the next step we will bring together the vortex generator and a high-lift airfoil in order to investigate the influence of the onflow disturbance on the airfoil stall behavior. In the hybrid RANS/LES simulation the ADDES-based simulation strategy for the high-lift airfoil proposed in Sec. 2.2 will be used. In the first setup that will be investigated, the vortex does not directly interact with the high-lift airfoil so that only its mean characteristics have an influence on the airfoil. Therefore, the vortex transport will be modelled in the RANS mode and we will make use of the spanwise grid adaptation. A coarse spanwise resolution will be used in the transport region, whereas only around the high-lift airfoil the grid will be refined in spanwise direction for HRLM.

In future investigations of a modified vortex-generator setup, where the vortex directly interacts with the high-lift airfoil, the URANS approach is considered insufficient to resolve the relevant scales of the vortex which interact with the airfoil boundary layer. In this scenario the vortex must be modelled with a scale-resolving approach. In this case it is necessary to investigate how the quality of the predicted resolved turbulence improves when, instead of the random forcing, the synthetic eddy method [2] that is available within the ADDES framework is used. It would also be worthwhile to include the full 3D wind tunnel in further RANS simulations to investigate the influence of the side walls on the vortex.

\section{Acknowledgement}

The authors greatfully acknowledge the Deutsche Forschungsgemeinschaft (DFG) that funded this work in the framework of the research group FOR1066.

Radespiel et al (Eds.): Advances in Simulation of Wing and Nacelle Stall, NNFM 131, pp. 41-54 Springer, 2015 


\section{References}

[1] Chauvet N, Deck S, Jacquin L (2007) Zonal Detached Eddy Simulation of a Controlled Propulsive Jet. AIAA Journal 45(10):2458-2473

[2] François DG, Radespiel R (2014) Airfoil Stall Simulations with Algebraic Delayed DES and Physically Based Synthetic Turbulence for RANS-LES Transition. AIAA 2014-2574

[3] François DG, Radespiel R, Reuß S (2014) Computations of Separated Flows with Hybrid RANS/LES Approaches. In: 4th Symposium "Simulation of Wing and Nacelle Stall", 1. - 2. Dec. 2014, Braunschweig, Germany

[4] Klein S, Hahn D, Scholz P, Radespiel R (2013) Vortex interactions with a highlift airfoil in a low speed wind tunnel. AIAA 2013-2875

[5] Knopp T, Probst A (2013) An Algebraic Sensor for the RANS-LES Switch in Delayed Detached-Eddy Simulation. In: New Results in Numerical and Experimental Fluid Mechanics VIII, Notes on Numerical Fluid Mechanics and Multidisciplinary Design 121, Springer Verlag, pp 457-464

[6] Probst A, Reuß S (2014) Scale-Resolving Simulations of Wall-Bounded Flows with an Unstructured Compressible Flow Solver. In: 5th Symposium on Hybrid RANS-LES Methods, TEXAS A\&M University, College Station, Texas, USA

[7] Probst A, Radespiel R, Wolf C, Knopp T, Schwamborn D (2010) A Comparison of Detached-Eddy Simulation and Reynolds-Stress Modelling Applied to the Flow over a Backward-Facing Step and an Airfoil at Stall. AIAA 2010-920

[8] Probst A, Radespiel R, Knopp T (2011) Detached-Eddy Simulation of Aerodynamic Flows Using a Reynolds-Stress Background Model and Algebraic RANS/LES Sensors. AIAA 2011-3206

[9] Radespiel R et al (2013) Simulation of Wing Stall. AIAA 2013-3175

[10] Reuß S, Knopp T, Schwamborn D (2012a) Hybrid RANS/LES simulations of a three-element airfoil. In: Progress in Hybrid RANS-LES Modelling Volume 117, Notes on Numerical Fluid Mechanics and Multidisciplinary Design, Springer Verlag, pp 357-367

[11] Reuß S, Probst A, Knopp T (2012b) Numerical investigation of the DLR F15 two-element airfoil using a Reynolds stress model. In: 3rd Symposium "Simulation of Wing and Nacelle Stall", 21. - 22. Jun. 2012, Braunschweig, Germany

[12] Reuß S, Wolf C, Knopp T, Raichle A, Schwamborn D (2012c) Chimera technique for transporting disturbances. International Journal for Numerical Methods in Fluids 70:1558-1572

[13] Reuß S, Knopp T, Probst A, Orlt M (2014) Assessment of Local LES-Resolution Sensors for Hybrid RANS/LES Simulations. In: 5th Symposium on Hybrid RANS-LES Methods, TEXAS A\&M University, College Station, Texas, USA, Notes on Numerical Fluid Mechanics and Multidisciplinary Design

Radespiel et al (Eds.): Advances in Simulation of Wing and Nacelle Stall, NNFM 131, pp. 41-54 Springer, 2015 
[14] Spalart PR, Deck S, Shur ML, Squires KD, Strelets MK, Travin AK (2006) A New Version of Detached-eddy Simulation, Resistant to Ambiguous Grid Densities. Theoretical and Computational Fluid Dynamics 20(3):181-195

[15] Travin AK, Shur ML, Spalart PR, Strelets MK (2006) Improvement of delayed detached-eddy simulation for LES with wall modelling. In: European Conference on Computational Fluid Dynamics, ECCOMAS CFD 2006, TU Delft, The Netherlands

[16] Wawrzinek K, Lutz T, Krämer E (2014) Numerical Studies of Turbulent Flow Influence on a Two-Element Airfoil. In: 4th Symposium "Simulation of Wing and Nacelle Stall", 1. - 2. Dec. 2014, Braunschweig, Germany

Radespiel et al (Eds.): Advances in Simulation of Wing and Nacelle Stall, NNFM 131, pp. 41-54 Springer, 2015 